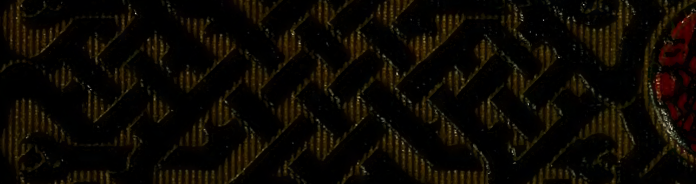
(5)

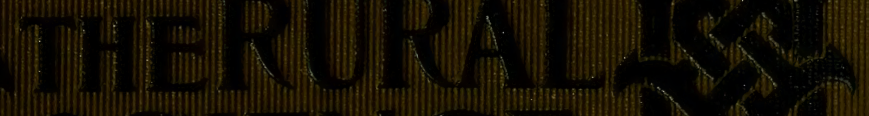
(it)
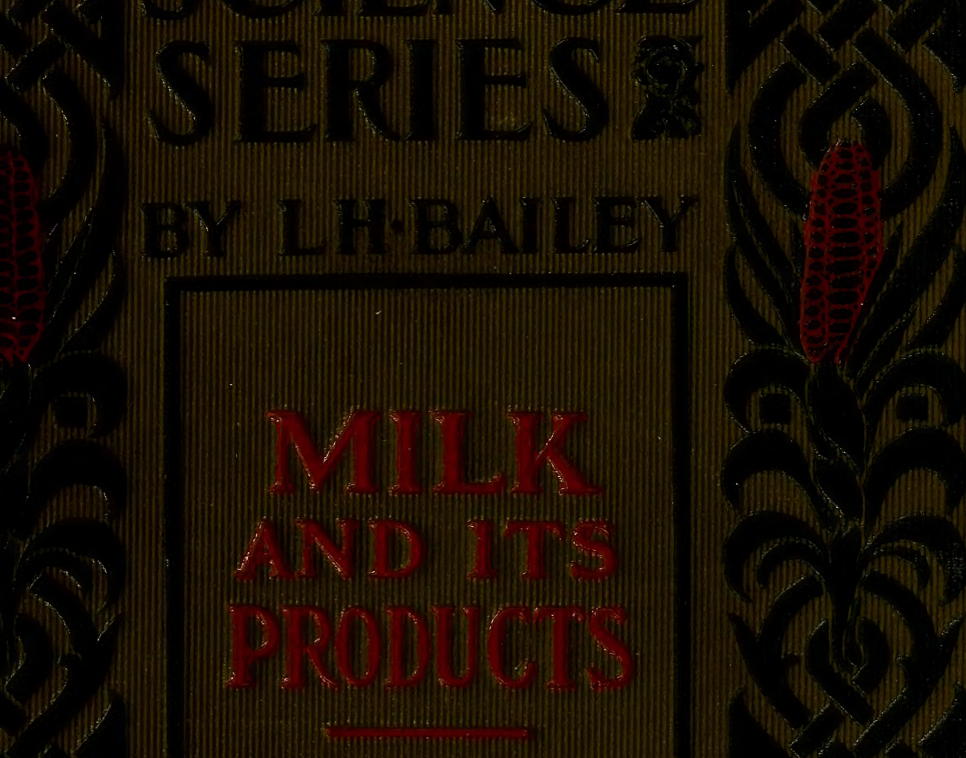

fin (I)
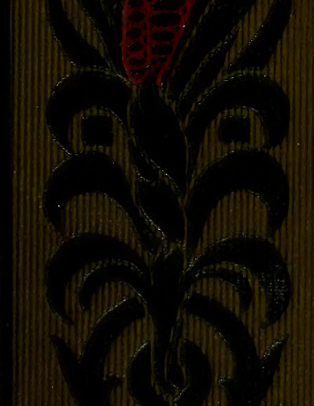

(1)

ation

i)

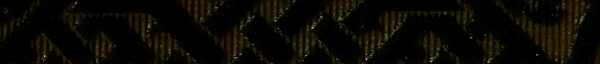

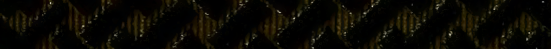

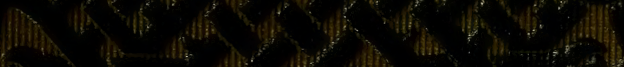

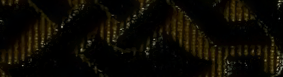





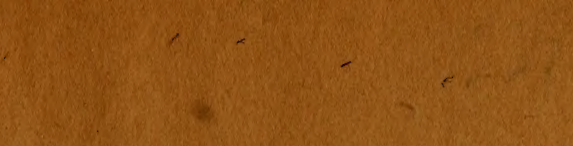

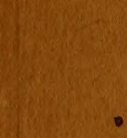

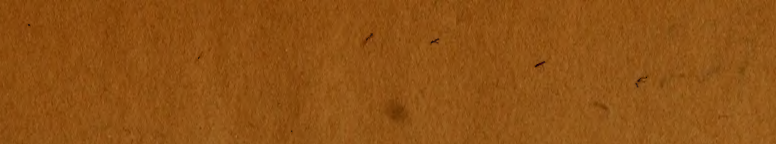

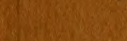

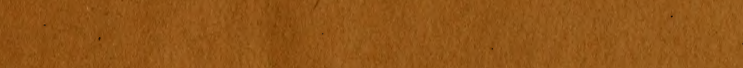

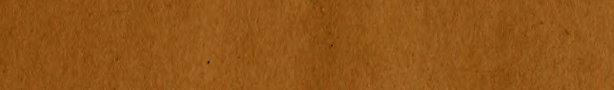

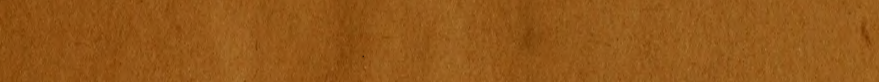

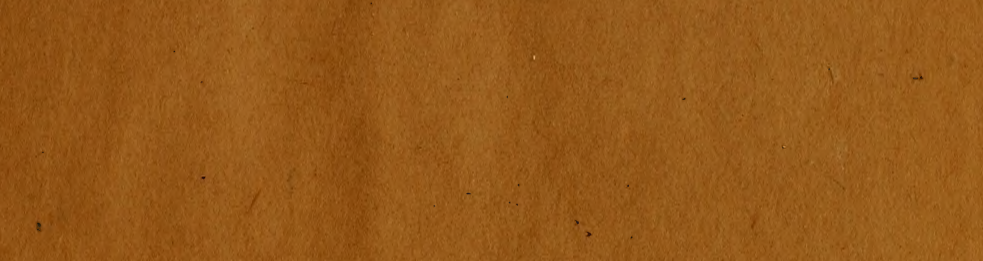

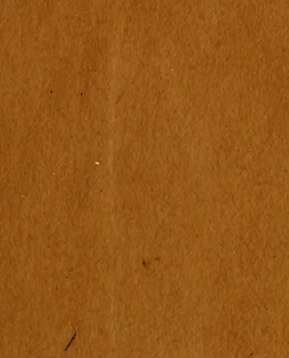

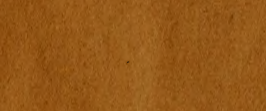

2.7.
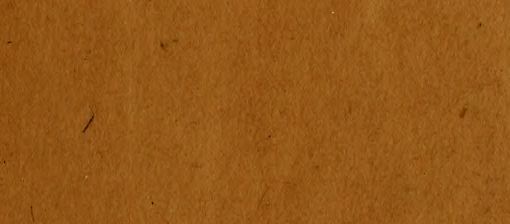

.

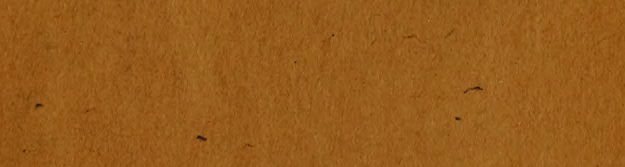

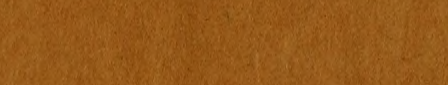





\section{The FRuxal Srience Sexies}

EDITED BY L. H, BAILEY

MILK AND ITS PRODUCTS 
The $>$ N 


\title{
MILK AND ITS PRODUCTS
}

A TREATISE UPON THE NATURE AND QUALITIES OF DAIRY MILK AND THE MANUFACTURE OF BUTTER AND CHEESE

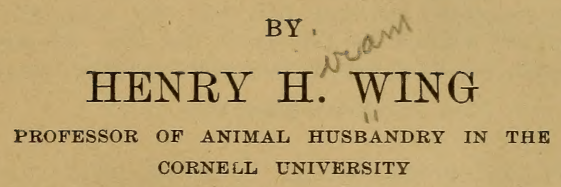

REVISED AND ENLARGED

\begin{abstract}
职ew 县ork
THE MACMILLAN COMPANY

LONDON: MACMILLAN \& CO., LTD.

1913

All rights reserved
\end{abstract}




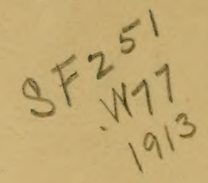

\section{COPYRIFHT, 1897 AND 1913 \\ By HENRY H. WING}

Set up and electrotyped February, 1897

Reprinted with corrections February, 1898, July, 1899, July, 1900. January, 1902, February, 1903. January and September, 1904, July, 1905, April, 1906;

August, 1907, June, 1908, January and July, 1909, July, 1911

New edition, 1913 


\section{Tro $\mathfrak{M}$ w Fathex}

AS A SLIGHT TRIBUTE TO THE CAREFUL TRAINING AND WISE AND SYMPATHETIC COUNSEL THAT

SERVED TO INSTIL IN THREE FARM BOYS A LOVE FOR ALL THAT PERTAINS TO FARM LIFE

This little waxk is affectionately insexibed 



\section{PREFACE}

THE revolution in dairy practice brought about by the introduction of the centrifugal cream separator and the Babcock test for fat and by a more definite knowledge regarding the various fermentations that so greatly influence milk and the manufacture of its products, has seemed to demand the publication of a small handbook that shall give to the dairyman, and particularly to the dairy student, in simple, concise form, the principles underlying modern dairy practice. In attempting to meet this demand, I have had largely in view the needs of my own students, while still keeping in mind the general dairy reader.

In the collation of the information, where so many points are still unsettled, it is of course difficult in all cases to distinguish fact from conjecture. The aim has been at all times to give the present state of knowledge as supported by the weight of evidence and the opinions of those whose authority is highest. In how far this has been successful time alone can tell. It would be (vii) 
too much to hope that every conclusion will stand the test of further investigation and experience.

Dairy practice in the United States owes much to the investigations of the Agricultural Experiment Stations. Of the results of their labor free use has been made in various ways, and in many cases without specific mention at the particular place. Without wishing to make distinctions, particular acknowledgment is here rendered to the reports and bulletins of the Stations in Maine, New Hampshire, Vermont, Connecticut (Storrs), Canada, New York (State), New York (Cornell), New Jersey, Pennsylvania, Illinois, Iowa, Wisconsin and Minnesota. For those who wish to make more extended investigations, a bibliography is added in the Appendix, giving references to many bulletins.

Thanks are due to D. H. Burrell \& Co., the Vermont Farm Machine Co., the DeLaval Separator Co., the Star Milk Cooler Co., the Champion Milk Cooler Co., J. F. Hodgkin, and F. B. Fargo \& Co., for the use of electrotypes.

Acknowledgment is also due my colleagues, Messrs. Cavanaugh, Durand, Hall and VanWagenen, for valuable assistance, and to Professor L. H. Bailey for much friendly counsel and many useful suggestions.

Cornell University Dairy,

HENRY H. WING.

January 1897. 


\section{PREFACE TO THE NEW EDITION}

IN THE preparation of a new edition of Milk and Its Products, in addition to making such changes as are necessary to bring the body of the work up-todate, it has seemed well to add chapters on dairy cattle and the production of milk, on certified milk, and on ice-cream manufacture. Brief directions for simple bacteriological determinations have also been added. The latter has been prepared by my daughter, Lois W. Wing, late assistant in dairy bacteriology, in the New York State College of Agriculture. The chapter on certified milk was written by Mr. George C. Watson, formerly manager of the Tully Farms; and grateful acknowledgment is hereby made to both for their assistance.

\section{HENRY H. WING.}

New York State College of Agriculture

Cornell University, July, 1912 



\title{
CONTENTS
}

\author{
CHAPTER I \\ Secretion of Milk
}

Milk defined-Mammary glands-Udder-Internal structure of udder and teats-Ultimate follicle-Secretion of milk- Incentives to secretion-Amount and duration of flow-Effect of succeeding pregnancy-Incomplete remioval of milk-Regularity and frequency of milking-Control of animal over secretion.

Pages 1-15

\section{CHAPTER II}

\section{Composition of MilK}

Milk constituents-Colostrum-Specific gravity-The fats-The volatile fats - The non-volatile fats-The albuminoids-The sugar-The ash-Other constituents-Variations in quality of milk.

Pages 16-34

\section{CHAPTER III}

\section{The Production of Milk-Dairy Cattle}

Milk a maternal function-The cow the only commercial milk producer-Factors in the production of milk-The lactation period-The operation of milking-Relation of form to capacity-Value of records of production-Necessity for keeping records-Food as a factor in milk production-The ideal ration-Selection of breed-Pure breds and grades-Maintenance of the dairy herd-Selection of the bull-Management of the bull-Grading up the herd-Major and minor dairy breeds-Jerseys-Guernseys - Holstein-Friesians - AyrshiresShorthorns - Red Polled-Brown Swiss - Dutch Belted Devons.

Pages $35-76$ 


\section{CHAPTER IV}

\section{The Testing of Milk}

Gravimetric anaylsis-History of milk tests-Cream gaugesSpecific gravity-Lactometers-Churn tests-Lactobutyrometer-Pioscope-Lactoscope-Soxhlet's Method-Lactocrite -Fjord's control apparatus-Milk tests in the United StatesShort's method-Method of Failyer and Willard-Parsons' method-Iowa Station test-Cochran's method-Babcock test -Beimling test-Gerber's method-Butyrometer-Details of Babcock test-The centrifugal machine-The glasswareSampling the milk-Composite sampling-Making the testThe acid-Whirling-Reading-Cleaning the glassware.

Pages 77-107

\section{CHAPTER V}

The Ferments and Fermentations of Milk and Their Control

Tendency to undergo change-Germs of fermentation-BacteriaPresence of bacteria in milk-Kinds of bacteria in milkFermentations of milk-Relation of milk bacteria to the human system-Lactic fermentations-Fermentations affecting the albuminoids-Butyric fermentations-Control of fermentations-Prevention of infection-Holding at low temperatures-Destruction of germs in milk-PasteurizationSelection of milk for pasteurization.

Pages 108-124

\section{CHAPTER VI}

\section{Determination of Bacteria in Milk}

Bacteria as a measure of dirt in milk-The laboratory-Apparatus Media - Sterilization-Cleaning glassware-Procedure for plating-Checks.

Pages 125-134

\section{CHAPTER VII}

\section{MARKeT MiLK}

Milk for consumption-Cleanliness-Treatment after drawingAëration-Delivery-Bad flavors in milk-Quality of milk for consumption-Control of milk supply-Cream for consumption-Pasteurized cream-Quality of cream. 


\section{CHAPTER VIII}

\section{Certified Mrlk}

Definition-Origin of certified milk-Standards-ProductionSanitary stables-Selection of the cows-Care of the cowsCare of the stable-Milking-Care of utensils-Cost of production.

Pages 149-162

\section{CHAPTER IX \\ Separation of Cream}

Gravity creaming-Shallow-pan creaming-Deep setting systemCentrifugal separation-Conditions affecting completeness of separation-Conditions affecting the relative amount of skimmed milk and cream-Contrivances in the bowl to increase the efficiency of separation-Mechanical conditions affecting separation-Efficiency of separation in centrifugal machinesDesirable and undesirable features of a separator.

Pages 163-194

\section{CHAPTER X}

\section{The Ripening of Cream}

Means of producing lactic acid-Temperature of ripening-Amount of acid necessary-Acid tests-Determination of lactic acid. in milk and cream-Further effects of ripening-Churning cream of different degrees of ripeness-Bad effects of overripening.

Pages 195-209

\section{CHAPTER XI}

\section{Churning}

Viscosity of the milk-Ripeness of cream-Temperature-Nature of agitation-Quality of the globules of fat-End of churning-Difficult churning.

Pages 210-219

\section{CHAPTER XII}

Finishing and Marketing Butter

Washing the butter-Working-Salting-Brine salting-Packing and marketing - Composition and quality of butter.

Pages 220-232 


\section{CHAPTER XIII}

\section{Milk for Cheese Making}

Theory of cheese making-Quality of milk for cheese makingLoss of fat-Cooling-Aëration-Ripening-Rennet testsDegree of ripeness necessary-Starters-Rennet-Removal of whey.

Pages 233-250

\section{CHAPTER XIV}

\section{Cheddar Cheese Making}

Setting - Cutting - Heating - Cheddaring — Grinding — Salting Curing-Difficulties likely to occur in cheddar cheese making-Qualities of cheese.

Pages 251-268

\section{CHAPTER XV}

\section{Other Varieties of Cheese}

Home-trade or stirred-curd cheese-Sage cheese-Young America-Picnics - Pineapple - Truckle-American Neufchatel Philadelphia cream cheese-Limburger-Imitation SwissPrepared cheese-English cheese-Stilton-Cheshire-Lancashire - Derbyshire - Leicestershire - Wensleydale - Gorgonzola-Emmenthaler or Swiss-Edam-Gouda-RoquefortBrie-Camembert-D'Isigny-Pont L'Eveque-Port du Salut -Parmesan.

Pages 269-298

\section{CHAPTER XVI}

\section{ICE CREAM}

Relation to dairy practice-Classification-Quality of creamSugar-Flavors-Fillers-Freezing and packing-Transferring -Freezers-Recipes-Scoring.

Pages 299-314

\section{CHAPTER XVII}

Other and By-Products of the Dairy

Skimmed milk, buttermilk and whey-Condensed milk-Dried casein-Milk sugar-Dutch cheese-Whey cheese-Cheese food-Koumiss-Kephir-Wheyn.

Pages $315-325$ 


\section{CHAPTER XVIII}

\section{Butter and Cheese Factories}

Location of creameries-Arrangement of building-Construction-Cheese factories-Combined butter and cheese factories-Farm dairy buildings.

Pages 326-337

\section{CHAPTER XIX}

Statistics AND Economics of the DaIRy Industry

Increase in dairy production-Development of the factory system-Dairy legislation-Dairy markets.

Pages 338-347

\section{APPENDIX}

A. Useful rules and tests.

B. Metric system of weights and measures.

Pages 349-362

Pages 363-364

C. Legal standards for milk in the various states-The oleomargerine law-The filled-cheese law-The New York state dairy law.

Pages 365-403

D. References to Agricultural Experiment Station reports and bulletins.

Pages $404-415$

INDEX

Pages $417-433$ 

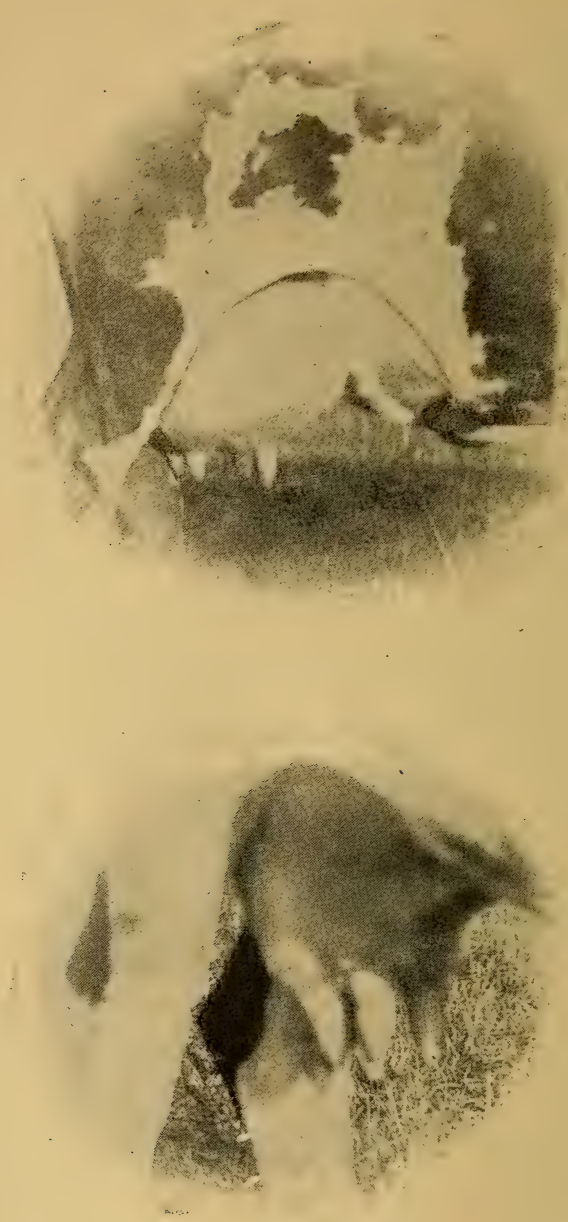

Udders of good and poor types.

Prom Bulletin No. 62, Purdue Univ. Agr. Exp. Sta., by permission. See page 3. 


\section{MILK AND ITS PRODUCTS}

\section{CHAPTER I}

\section{THE SECRETION OF MILK}

THE females of all animals that suckle their young (class Mammalia) secrete for this purpose a special fluid which is known as milk. It is an opaque yellowish white fluid, with a slight alkaline reaction and a faintly sweetish taste. It consists of an emulsion of fats in a watery solution of alkaline salts, casein and sugar. It is secreted in two special glands situated without the body cavity ori either side of the median line, and known as the mammary glands or mammæ.

Mammary glands.-While, strictly speaking, there are but two glands, each gland may be divided into two or more lobes, each having a separate opening; thus, while there are ordinarily but two simple glands in the ewe, mare and goat, in the cow there are four or six, in the cat and bitch six to ten, and in the sow ten to fourteen. In animals having multiple glands, the mammæ occupy nearly the whole of the lower part of the chest and abdomen. In ther animals the glands are confined either to the nest or abdomen. In many animals each gland 
or lobe is furnished with a single opening connecting with a single duct, in others several ducts open independently upon the surface of a single nipple or teat. The mammary gland is a true organ of secretion in the sense that its product (milk) contains substances not before existing in the blood, that are formed during the process of secretion in the gland itself. In the cow the mammary glands are located on the posterior portion of the abdomen between the hind legs, and each gland is made up of two lobes or quarters, each having a single outlet furnished with a single duct, though there are often one and sometimes two rudimentary ducts upon the rear quarters, and which are occasionally developed to such an extent that milk may be drawn from them in small quantities. The whole organ is spoken of as the udder, and the ducts as teats. While the mammary gland is essentially a female organ, it is present in a rudimentary condition in the males of all mammals, and in exceptional cases in man and in the lower animals the organs of males have developed to such a degree as to secrete milk.

The cow's udder. - The udder is enclosed in a fold of skin, which is here thinner and softer than upon other parts of the body, and is supported by a band of fibrous tissue that springs from the median line of the body and extends through the whole substance of the gland. It varies very much in size and shape in different animals and in the same animal at different times. Its size is not always an indication of the secreting powers of an animal, since the num- 
ber of true secreting follicles does not necessarily bear any relation to the apparent size. The udder in a good cow should be large and well developed; it should occupy the whole space between the hind legs, extending well up between the thighs and well forward upon the belly. It should be held firmly against the wall of the abdomen. It should be level or nearly so on the bottom, and the four quarters should be as nearly as possible equally developed and each furnished with a cylindrical perpendicular teat of moderate length. The whole organ should diminish rapidly in size as the milk is withdrawn. The hair upon the udder should be fairly abundant, fine and soft, and abundantly supplied with a brownish dandruff.

The substance of the udder is composed of the fibrous band, already mentioned, connective tissue, fatty tissue, milk ducts or canals, true secreting cells (acini, ultimate follicles, alveoli), veins, arteries, nerves and lymphaties, the whole making up a reddish gray mass of spongy texture.

The udder varies very much, in different individuals, in size and shape as well as in internal structure and secreting capacity. In some animals the amount of connective and fatty tissue is much larger than in others. Such udders are said to be "fleshy," and while usually of large size and good shape, are deficient in true secreting eapacity. They are firm to the touch, particularly when empty, and do not markedly diminish in size when the milk is withdrawn. It is generally supposed that such 
udders are more subject to inflammations and inflammatory diseases than those with less fatty tissue. In many cows the fibrous net-work that supports the udder is held firmly up to the under side of the abdomen. If, in connection with this, the udder has comparatively little connective and fatty tissue, the animal will have an udder apparently small, but with large capacity for secretion. In old cows, particularly those that have been large milkers, the fibrous bands often become largely relaxed, so that the udder falls nearly to the ground, and appears to be of enormous size.

Internal structure of the udder and teats. - The teat is simply a canal surrounded by muscular walls and closed at the extremity by an involuntary sphincter muscle, which varies much in rigidity in different animals; often it is so lax that the pressure of a small amount of milk in the canal is sufficient to open it and the animal leaks her milk. In other animals it requires a strong effort of the hand to draw the milk. When desirable, the rigidity may be overcome by keeping a smooth wooden plug of sufficient size to moderately dilate the opening in the end of the teat till the muscle relaxes sufficiently to permit easy drawing of the milk, or the muscle may be partially divided with the knife in the hands of a skilful operator. At the top of the teat, or bottom of the udder, there is a small cavity known as the milk eistern, serving to hold the milk after its secretion until it is drawn. It is of varying capacity, up to half a pint, 
and is partially separated from the canal of the teat by a more or less well-marked constriction in the muscular walls of the upper part of the teat. From the milk cisterns a system of canals or so-called milk ducts extends to all portions of the udder. These ducts are larger near their opening into the milk cistern, and diminish in size as they rise through the udder. They branch and anastomose freely in all directions, and finally end in a group of small sac-like bodies, the ultimate follicles. The system of milk ducts arising from each teat is practically distinct, though there is more or less communication between the smaller ducts in the upper portions of the two quarters on the same side of the animal. This renders it possible to draw a part of the milk secreted in the hind quarter from the forward teat on the same side, and vice versa. There is no communication between the ducts on opposite sides of the animal. At the junctions of the larger ducts there are greater or smaller enlargements, forming small cavities or milk reservoirs, which serve the same purpose as the reservoirs at the top of the teat. The branching points of all the ducts, large and small, are guarded by sphincter muscles. These muscles are connected with the abdominal muscles of the animal, and she is able to more or less completely close them at will. and so "hold up" her milk. It requires a strong effort on the part of the animal to completely close the larger ducts in the lower part of 
the udder; a comparatively slight effort is all that is necessary to close the smaller vessels. Animals vary greatly both in the control they possess over these muscles and in their disposition to use it. Very few can completely close the larger ducts, and very many rarely exercise whatever power they do possess. Sudden fright, the presence of strange persons or animals in the stable, any irregularity in the time or manner of feeding or milking, and slight feverish conditions, particularly sexual heat, are the most common provocatives to holding up milk. There are very many cows that contract the habit of holding up the milk upon the slightest provocation, and if the habit is once formed it is almost impossible to cure it, and the result is that the usefulness of the animal as a milk producer is largely destroyed, for the retention of the milk in the udder interferes greatly with the activity of secretion, and in a short time permanently lessens it.

The ultimate follicles.-The milk ducts, after branching and anastomosing in all directions, finally end in a group of small sac-like bodies known as acini, or ultimate follicles. It is in these small bodies that the secretion of the milk takes place. They are about 1-30th of an inch in diameter, and are found in groups of three to five, with a common outlet at the end of each branching duct. In form and appearance they present marked changes according to the condition of the animal. During active lactation they are found in their highest development. When lactation ceases, the 
smaller ducts become much retracted, and the follicles shrink in size and finally become rudimentary, or even entirely disappear, until under the stimulus of a succeeding pregnancy, the whole gland renews its activity, and the ducts and follicles regain their former size and appearance. New ducts and follicles may also be formed up to about the fifth or sixth year, and the power of the animal to secrete milk be thereby increased. Surrounding the follicles, and intimately attached to them, are capillary blood vessels, both veins and arteries, and through the cells of the membranes making up the walls of all these vessels the fluids of the blood freely pass into the cavity of the follicles by means of osmosis, or transudation. The cavity of the follicle is lined with epithelial cells, that during lactation are filled with protoplasm, and are capable of rapid multiplication, growth, and degeneration, at the same time that the cell contents are undergoing rapid and extensive changes.

The secretion of milk. - The milk is formed from the blood, partly by the transudation of the blood serum directly into the cavity of the milk follicle, and partly by a transformation of the contents of the epithelial cells lining the cavity of the follicle, which at this time are especially active. The water passes directly from the capillaries into the milk follicles and ducts, carrying with it the mineral constituents in solution and a part of the albumin of the blood serum; but by far the larger 
part of the albumin is in some way changed during its passage from the capillaries, and appears in the cavity of the follicle as the casein of the milk.

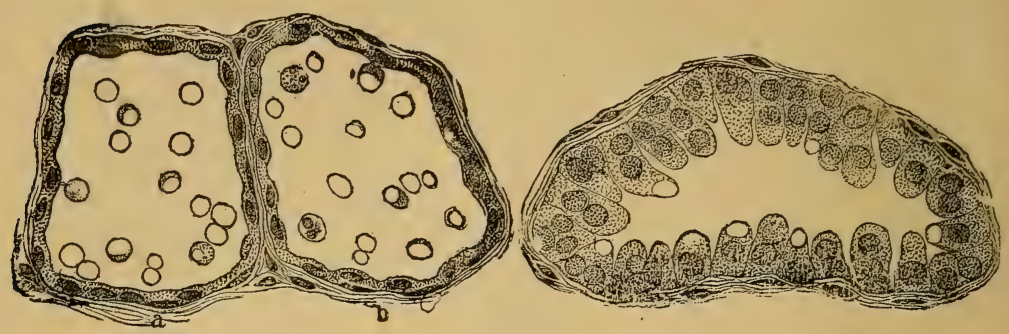

Fig. 1. Section through alveoli of the mammary gland of the dog in first and second stages of secretion. From Meade Smith, after Heidenhain.

When milk is being secreted, the lining cells of the follicle are in a state of constant activity. New cells are constantly being formed by budding or fission (the cell elongates, a partition forms across it, and the two halves so divided enlarge to the size of the mother cell, and there are two cells where but one existed before), and older cells are as constantly breaking down. While this is going on, the cell contents, consisting mainly of protoplasm, become changed into a globule of fat, and the globules so formed are either extruded through the cell wall into the cavity of the follicle, or set free by the breaking down and reabsorption of the cell wall. In all probability both processes take place. Small portions of the fat may also be carried over directly from the blood and appear in the milk without change. The milk sugar is probably formed 
through a chemical change in the contents of these lining cells, since but minute quantities of sugar are found in the blood.

Incentives to secretion.-Maternity is the prime incentive to the secretion of milk. While there is a distinct increase in the development of the mammæ upon attaining puberty, it is not until pregnaney is well advanced that the organ attains anywhere near its full development, or that there is any activity in the true secreting cells. In the virgin animal, and up to within a short time of parturition, the cavities and ducts of the udder contain a watery saline fluid, but true milk does not appear until a short time before, and in some cases not until after, parturition. The immediate stimulus to the production of milk is the turning of the blood that went to nourish the foetus from the arteries of the uterus to the arteries of the udder. The pressure of blood in the vessels of the udder stimulates the secreting cells to great activity, and the cells, hitherto dormant, begin to multiply rapidly. When this activity is first set up, the various processes of secretion are more or less incomplete, so that the milk first se creted is very different in character from that secreted afterwards, and is known as colostrum. The colostrum contains in the first place considerably less water than normal milk; in the second place, the transformation of albumin into casein is only partial, so that colostrum contains large amounts of albumin; and finally, when secretion of milk begins, the cells of the follicle multiply more rapidly than 
they can be reabsorbed, and portions of partially broken down cells break away from the walls of the follicle and appear in the colostrum. Gradually the colostrum takes on the character of normal milk, and in the course of four or five days the change is complete. Other characteristics of colostrum are discussed in detail in the next chapter.

While maternity is the prime cause of secretion, it is not the only means of stimulation to the activity of the udder, nor is it a necessary prerequisite to the secretion of milk. The regular removal of the saline fluid in the gland of the virgin animal, or even the stimulation of the organ by the friction of the hand or the suckling of a calf, may be sufficient to cause the secretion of milk of normal character in considerable quantities. In the same way and under the same exciting causes, other glands of the body, notably the lymphatics in the arm pits and the rudimentary mammæ of males, have been known to secrete a fluid resembling milk in all essential characteristics.

Amount and duration of flow.-With wild animals in a state of nature, the milk is secreted only in amount sufficient for the needs of the young animal, and only until the young is sufficiently developed to secure its food independently of the mother. Under the influence of domestication the cow has been brought to increase her flow of milk many fold, and the time during which it is secreted has been lengthened until it is almost, and indeed is, in some cases quite continuous. A äis- 
cussion of the agencies by means of which this most important result has been brought about would open up the whole question of the selection, breeding and training of cows, as well as everything pertaining to the science of foods and feeding, which is not here possible. There are, however, some physiological conditions affecting the secretion of milk that may be mentioned.

Milk is secreted from the blood. The amount of milk secreted will, therefore, depend upon the amount of blood passing through the udder, and this, in turn, will depend upon the number and size of the blood vessels, not only in the udder itself, but leading to it and away from it, the vigor of the circulation, the supply of food to the animal, and her capacity to eat, digest and assimilate it and turn it into blood. From or shortly after parturition, there is a constant tendency of the blood vessels in the udder to shrink in size, and consequently a constantly diminishing flow of milk. When the period of lactation advances at the same time that the pastures are growing more scanty and less succulent, this diminution is fairly regular and constant, especially after from three to five months of the period of lactation have passed. This tendency to shrinkage in the size of the blood vessels of the udder may be held in check in great measure by an abundant supply of nutritious food, particularly if it is of a succulent character, and it is not at all uncommon to find cows secreting milk in regular amount, or "holding out," for eight 
or ten months. But in this respect the individuality of the animal plays an important part, so that wide variations are seen in different individuals under the same conditions of food and care. After a shrinkage in the flow has once taken place, it is extremely difficult to again increase it by increased food until after another calving.

Effect of succeeding pregnancy.-The effect of the animal again becoming pregnant is to decrease the flow of milk. The cause of this decrease seems, in many cases, to be two-fold: First, a sympathetic effect, following immediately upon conception, and secondly, a shrinkage due to a turning away of a part of the blood from the udder to nourish the growing foetus. This shrinkage does not become marked until the fourth or fifth month of pregnancy. In this respect, as in their power to "hold out," individual animals show the widest variation. With very many the effect of becoming again pregnant is so slight as to be scarcely noticeable; with others it is so great as to materially interfere with the usefulness of the animal.

Incomplete removal of milk.-One of the most important means of checking the secretion of milk lies in the incomplete removal of milk already secreted. We have already seen that the removal of the saline fluid from the ducts of the inactive gland is an efficient stimulus to secretion. So, too, the presence of milk in the ducts acts as a check to further secretion. Further than that, it not only checks secretion but is an actual irritant, suff- 
cient in many instances to give rise to inflammations of a serious character. Clean milking is one of the most important aids in keeping up and prolonging the flow of milk.

Regularity and frequency of milling.-While the process of milk secretion is a continuous one, it is not entirely uniform. There is reason to believe that the secretion is considerably more rapid while the operation of milking is going on, and that a considerable portion of the whole amount is then secreted. On the other hand, the distension of the milk ducts and reservoirs by milk already secreted acts as more or less of a check upon the activity of the follicles, and so lessens the rapidity of secretion. While for these reasons it would be inferred that frequent milking would lead to increased secretion, the limits of such increased secretion are moderate, and beyond a certain point no increased flow of milk is secured by increasing the frequency of the milking periods. In all cases where the udder becomes unduly distended with milk between periods, an increased flow will be secured by milking oftener. The common practice is to milk twice in the twenty-four hours, and the nearer the time can be divided into equal reriods the more uniform will be the secretion. Where more frequent. millking is practiced the same principle will hold. Not only is regularity in the period from morning to night of importance, but regularity in the time of milking from day to day is equally so. A difference of an hour in the time of milking will frequently make a difference of 10 per cent in the 
amount secreted, and where these irregularities are frequent, will soon result in a considerably diminished flow. The amount of milk given is also considerably affected by the way in which the milk is drawn. In general, it may be said that rapid milking is conducive to a large flow. In any event, the milk should be drawn so that no discomfort is eaused the animal, and in this respect there is great difference in milkers. A rapid, uniform stroke, with a firm touch on the teat, and a stroking motion to the lower part of the udder, gives the best results. Babcock has found that certain milkers uniformly get not only more but richer milk from the same cow.

Control of the animal over secretion.-The secretion of milk is involuntary. The animal can no more control it than it can control the respiration or the circulation of the blood. Yet there are numerons conditions of the animal that have a more or less direct effect upon the secretion of milk. These conditions have not only to do with the physical condition of the animal-as the supply of food, the circulation of the blood, extremes of temperature, etc.-but extend in large measure to the nervous organization and condition of the animal. We have already seen that the animal may by an exercise of will more or less completely control the withdrawal of milk already secreted; so, too, there are nervous conditions that have a great effect upon the actual secretion. Sudden fright, an unfamiliar milker or attendant, unusual excitement, sexual excitement, or the presence of an animal in heat in the herd, an 
unusual amount of exercise, or any one of many other causes, may be sufficient to decrease the secretion of milk one-half in any one day. The effect of such disturbances is usually quickly overcome, but their frequent recurrence leads to a permanent diminution of the secretion. The nervous organization of the animal is a most important factor in determining the effect of these various disturbing influences. Many of the animals in which the powers of digestion, nutrition and secretion are most highly developed are possessed also of a highly developed and sensitive nervous system, and hence are easily affected by any disturbing condition. With all such animals it is of the utmost importance that every condition surrounding the animal should be as regular. and uniform as possible. Other animals of equal capacity show a remarkably quiet and phlegmatic nervous temperament, and are consequently slightly or not at all affected by such disturbing influences. Such animals are of great value to the milk producer, for with the utmost care and regularity there are always disturbing influences beyond the control of the dairyman. 


\section{CHAPTER III}

\section{COMPOSITION OF MILK}

THE constituents of milk are numerous and of diverse character, but may be easily classified into a few well marked groups as follows: (a) water, (b) fats, (c) substances containing nitrogen (albuminoids), (d) sugar, (e) ash. Excepting the water, they are collectively known as milk solids. The solids exist partly in solution, partly in semi-solution, and partly in suspension in the water. Milk from the various classes of animals has the same general constitution and properties, and varies only in the relative proportions of the various proximate constituents. Cow's milk is typical of all milks, and as it is the only milk used in processes of manufac. ture in the United States, all of our discussions have reference to it alone. In various other countries milk from the goat, ass, mare and ewe is considerably used, not only as food but for the manufacture of various products. Indeed the peculiarities of some of these are supposed to be largely due to their having been made from the milk of animals other than the cow. Koumiss, made from mare's milk in Arabia, and Roquefort cheese, made largely from ewe's milk, are noteworthy examples. 
All of the milk constituents are more or less variable in quantity, and many of them vary widely: hence it is not possible to make a statement of the average percentage composition of milk that will give more than a general idea of its composition. The following are taken from recent authorities in the various countries:

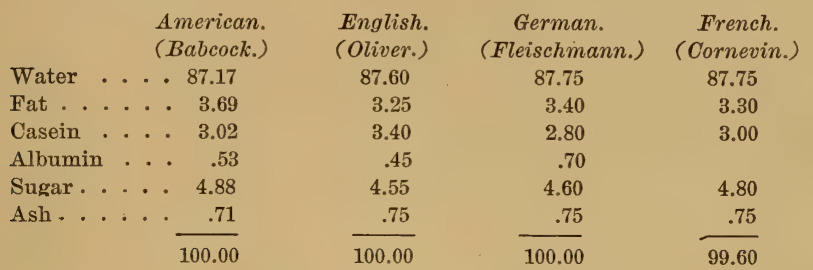

The following, from Kœnig, shows the range of variation of the several constituents in nearly 800 analyses collected from all parts of the world:

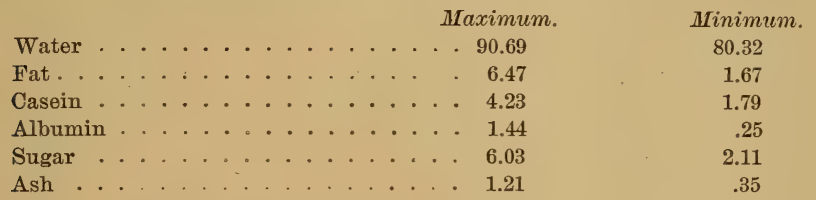

While the range of variation shown above is considerable, some of the constituents, notably the fat, may show even greater ranges in milk secreted by normal, healthy cows. It is probable that the minimum of Kœnig is seldom exceeded, but as high as 10 per cent of fat has been found in the milk of single cows giving a very small quantity. Babcock states that no analysis showing more than 9

B 
per cent of fat is recorded from any cow giving as much as 15 pounds of milk per day. Any analysis above 7 per cent is extremely rare, and should be regarded with suspicion unless well authenticated. The mixed milk of herds seldom falls below 3 per cent of fat and rarely rises above 5.5 per cent.

Colostrum.-The first milk secreted by the animal after parturition is quite distinct in composition and physical properties from that produced after the secretion has become well established. Such milk is called colostrum, and is ordinarily considered unfit for consumption or manufacture. Colostrum differs from normal milk chiefly in its less proportion of water and sugar, in the much greater proportion of albumin and ash, in the extremely variable amount of fat, and in the presence of small organized bodies known as colostrum corpuscles, which are probably debris of the cell structure of the gland. The following analyses from Richmond after Vaudin show the extremely variable composition of colostrum:

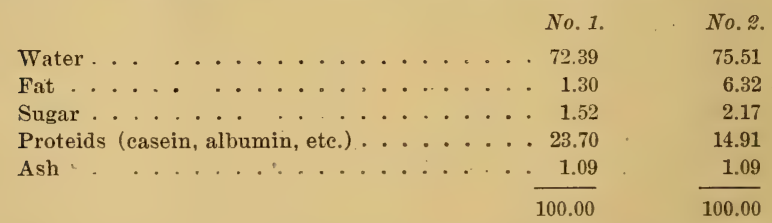

The percentage of albumin in colostrum is so great that it will cause the whole mass of milk to thicken upon boiling, and this is ordinarily considered a sufficient test for determining the suitability of the milk for consumption or manufacture. With- 
in four or five days after calving, the milk loses its coiostrum character and takes on its normal condition. This change is a gradual and progressive one, and is more or less dependent upon the physical condition of the animal. When the cow is feverish, or when there is local inflammation in the udder, the colostrum character of the milk is retained for a longer period than otherwise. The amount of coloring matter present is also considerably greater in colostrum than in normal milk, and the percentage of fat varies very widely. Usually the percentage of fat is less in the colostrum than in the normal milk from the same cow, although occasions are not infrequent where more fat is found in the milk immediately after calving than at any other time in the whole period of lactation.

Specific gravity of milk. - Some of the solids of milk are heavier than water and some of them lighter, milk as a whole having a specific gravity somewhat greater than water. The variation in the specific gravity is considerable, the range usually given being from 1.029 to 1.035 at $60^{\circ} \mathrm{F}$., the average being about 1.032. In general, the effect of an increase in the solids of the milk is to increase its specific gravity, though in milk extremely rich in fats (6 per cent or above) the specific gravity is lessened. Formerly, more than at present, it was the custom to estimate the quality of the milk by determining its specific gravity, but as soon as it became known that the specific gravity depended not so much upon the amount as upon the character of the solids, a 
determination of the specific gravity became of little value. Unscrupulous dairymen soon learned that water could be added to milk and fat or cream taken from it in such proportions that the specific gravity would remain the same as that of normal milk.

The fats. - The fat of milk, or butter fat, as it is often called, is a mixture of a considerable number of separate and distinct fats, no less than six or eight being normal to milk, and a considerable further number may be present under various conditions. The fats in milk are of two kinds, volatile and nonvolatile. To the former class belong the various normal essential oils that give to milk and butter their characteristic odors and flavors, and in addition to these normal fats there may be a large number of volatile oils that are present in the food of the cow, and that impart to the milk the characteristic flavors of such foods.

The volatile fats. - The volatile fats make up only a small part of the total milk fat; in general, probably about 15 per cent of the whole. The chief normal volatile fats are butyrin, caprin, caproin, caprilin and laurin. Of these, butyrin is in much the largest proportion and of much the greatest importanse. It is the chief volatile fat of milk and butter, and to it are due in large part the characteristic flavors and aromas of milk and butter. Butyrin readily decomposes, forming butyric acid, which is the chief elemer.t in the rancid or "frowy" taste that butter acquires upon long standing. 
The volatile fats that are derived directly from the food may give either desirable or undesirable flavors to the milk. Thus we esteem the characteristic flavors due to the grasses, clover and like fodder; on the other hand, the stronger flavors of garlic, onions, turnips, cabbage, etc., give to the milk an undesirable character.

The presence of these undesirable flavors in milk is often a source of a good deal of annoyance, but with proper precautions the bad results coming from them may be greatly lessened, and in many eases entirely obviated. Since all of these flavoring oils are volatile, they easily pass through all the tissues of the animal, and in a comparatively short time pass off through the various excretory channels. We shall find them present in the greatest amount not only in the milk, but in all the tissues of the animal, during the time that the fodder containing them is undergoing digestion, and by the time the digestion is completed the volatile products will have almost entirely passed away. If, therefore, sufficient care is taken to so time the feeding and milking that the milk shall be drawn not less than ten or twelve hours after the undesirable fodder has been eaten, there will be slight danger of contamination of the milk by it. Whereas, if milking occurs within three or four hours after feeding, the milk will be strongly impregnated with the undesirable flavor. Taking advantage of this, and feeding cows immediately before or immediately after milking, dairymen are often enabled to feed large quantities of turnips, 
and even onions, without danger of contamination of the milk. The presence of wild garlic and wild onions in pastures is a source of bad flavor in the milk in a considerable portion of the country. Where this is the case it is, of course, more difficult to overcome the bad flavor; but by allowing the cows to pasture for a comparatively short time only immediately after milking, and keeping them up and giving them some dry food for three or four hours before milking, there will be a great deal less annoyance from this source.

The non-volatile fats. - The non-volatile fats make up about 85 per cent of the whole amount of fat, and consist of a more or less uncertain and variable mixture of several fats, of which olein and palmitin make up the chief part. They are glycerides of the corresponding fatty acids-oleic, palmitic, stearic, myristic, etc., and differ from one another chiefly in their hardness or melting point. Olein is liquid at ordinary temperatures; palmitin and the others are solid. Olein melts at about $41^{\circ} \mathrm{F}$., the hard fats at various temperatures from $130^{\circ}$ to $150^{\circ} \mathrm{F}$. The mixture of the whole, as we find them in ordinary butter, melts at from $92^{\circ}$ to $96^{\circ} \mathrm{F}$. The hardness or softness of different butters, depending largely upon varying proportions of olein. Considerable doubt exists as to the relative proportions of the various fats and fatty acids. Browne* gives the following percentages of volatile and non-volatile fatty acids:

*Jour. Am. Chem. Soc. 21, 823. 


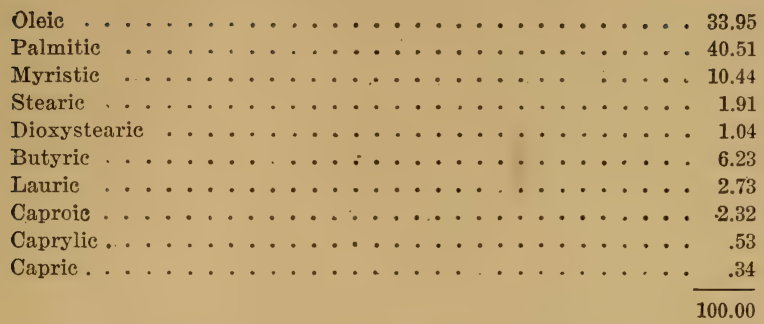

It is asserted that the coloring matter of the fat is most intimately associated with the palmitin. The fat exists in the milk in the form of an emulsion of extremely small globules, varying in size from $\frac{1}{1500}$ to $\frac{1}{25000}$ of an inch in diameter. These globules are not surrounded by pellicles, or socalled skins, as was formerly thought, but maintain their form by reason of the surface tension of the liquid fat, and also to some extent because of a layer of more or less condensed casein that immediately surrounds them. The permanency of the emulsion is further increased by the viscous nature of the milk serum, due to the presence of sugar and other solids in solution.

The albuminoids.-The casein is the chief albuminoid of milk, although there is always present a small amount of albumin, and, according to some authorities, of fibrin. Casein is of prime importance in the manufacture of cheese, and it is the chief constituent that goes to form tissue when milk is used as a food.

There is a good deal of doubt as to the form 
in which the casein exists. Formerly it was supposed that the casein is in solution. This idea was brought about by the fact that it is impossibls to filter the casein from the milk, even though it is passed through several thicknesses of fine paper. Later, though, it was found that when milk is passed through a fine porcelain filter a certain part is removed, and it was then supposed that there were two forms of casein, one of which was in solution and the other in suspension in very fine particles of a colloidal or gummy character. Still later investigations have shown that in all probability a large part of the casein is in this extremely fine colloidal state. When milk is subjected to the action of weak organic or mineral acids, to rennet or to certain vegetable substances, the casein is precipitated in a flocculent mass. Casein is not acted upon by heat. The albumin of the milk is in all respects similar to blood albumin. It is rendered insoluble by a heat of about $180^{\circ} \mathrm{F}$, but it is not acted upon by weak acids or rennet, and in this way it is chiefly distinguished from the casein. The fibrin of milk, if present, is in extremely minute quantities. It is supposed to be the same as blood fibrin, and coagulates upon exposure to the air, but is never present in sufficient quantity to form a clot, as in the case of blood. Its coagulation is hindered by a reduction of temperature, and it has been supposed that when it does coagulate it forms a sort of network of threads through the mass of milk. 
The sugar._Milk sugar, otherwise called lactose, exists in solution in the milk serum. It has the same chemical composition as cane sugar: that is, $\mathrm{C}_{12} \mathrm{H}_{22} \mathrm{O}_{11}+\mathrm{H}_{2} \mathrm{O}$. It crystallizes with considerable difficulty, and has very much less sweetening power than ordinary sugar. Under the influence of various ferments it readily undergoes decomposition, each molecule of sugar breaking up into four molecules of lactic acid. This change begins in the milk almost immediately after it is drawn, and continues until from .8 to 1 per cent of lactic acid is formed. The presence of lactic acid in this amount acts as a check upon the growth of the ferments, and prevents the further formation of lactic acid, unless the acid is nentralized with an alkali, when the fermentation proceeds as before. Milk sugar does not readily undergo alcoholic fermentation, but by the action of yeast and some other ferments the lactose is "inverted," or changed to dextrose and a peculiar substance known as galactose, and these readily change to alcohol under the influence of the proper ferments.

The ash.-The ash is the smallest and least variable constituent of the milk. It is composed chiefly of the phosphates of lime and potash, the chlorides of potash and soda, with small amounts of phosphate of iron and magnesia. Most of the salts are in solution. It seems probable that at least a part of the phosphate of lime is ordinarily in insoluble form, suspended in the milk in very fine particles in connection with the casein. The 
chloride of potash is largely in excess of the chloride of soda. This is exactly opposite to the proportions of these two salts in the blood.

Other constituents. - Besides the constituents enumerated above, several other compounds are more or less normally present in milk in minute quantities.

A small amount of citric acid is said to be a normal constituent of milk.

A peculiar substance called lactochrome is also a normal constituent of milk, and gives to it its characteristic color. This has been already mentioned in connection with the palmitin. The amount of lactochrome present varies under many conditions, notably the breed of the animal and the character of the food. Whatever other conditions may prevail, the milk is always of a higher color where the animals are fed on fresh green forage. This has led to the idea that the color of the milk is in some way connected with the condition of the chlorophyl or green coloring matter of the plant. Careno* has suggested that as the chlorophyl undergoes a change when the plant is dried, the digestive organs of the animal will have a different effect upon it, and so account for the difference in color in the milk.

An albuminoid called lactoprotein has also been described in milk.

Urea to the extent of .001 of 1 per cent may also be regarded as a normal constituent of milk.

* Milch Zeitung, vol. xxiv. 387. 
Variations in quality of milk.-We have already seen that the amount of milk secreted may vary greatly under the influence of a large number of varying conditions. So, too, we find that there are a large number of conditions that affect the quality of the milk, meaning thereby the relative proportion of the various constituents, and particularly the proportion of fat to other constituents. Some of these changes are regular and progressive during the period of lactation. Others are due to definite causes, and still others occur from time to time to which we have as yet been unable to ascribe any definite cause. After about the third or fourth week of lactation the percentage of fat in the milk remains nearly constant until the seventh or eighth month, or until the quantity of milk begins to rapidly diminish; but while the percentage of fat does not markedly change, the character of the fat undergoes several marked and characteristic changes. The butter globules are largest in size early in the period of lactation, and constantly diminish as lactation progresses, at the same time that they increase in number, so that the total amount of fat is not greatly changed. Early in the period of lactation there is a larger proportion of olein. In some cases it may amount to 50 per cent of the total fat. As the lactation progresses the proport:on of olein decreases and stearin and palmitin increases, until the proportion of olein may fall as low as 20 per cent. This change is more marked when the animal changes from fresh to dry food, as the 
period of lactation progresses. The hardening of the fat and the shrinking in the size of the globules are also more marked when the animal again becomes pregnant. In the case of cows that are milked for a prolonged period, as sometimes happens with farrow and spayed cows, the milk often becomes abnormally rich, not only in fat, but in casein; and in such cases the fat is usually made up of very minute globules.

It is usually observed that milks drawn at night and morning differ quite widely in the percentage of fat. This is not because there is any difference in the milk secreted by night or by day, although when cows lie still there is a larger percentage of water and a correspondingly less percentage of solids in the milk. The difference in the milk drawn at morning and evening is due to the unequal time that elapses between the periods. In general, the milk is richest in fat that is drawn after the shortest period, and this has been shown to be the case where cows have been milked three or four and even five times per day. It is, however, not an invariable rule that the milk is richest succeeding the shortest period. Not infrequently it has been found that the milk is richer after the longer period. In a series of observations made by the writer upon 12 cows, extending over 221 days, in 72 cases the percentage of fat was greater in the morning; in 114 cases it was greater in the evening, and in 35 cases there was a difference of .1 of 1 per cent or less 
between the morning and the evening milk. In this instance the period from evening to morning was about two hours longer. The amount of variation that may oceur between the morning and evening milk is often very considerable. In the great majority of cases it is not more than .5 of. 1 per cent, but variations so great as 2 or 2.5 per cent between the milk of one morning and that of the preceding or following evening have frequently been noticed. It is probable that a part of this variation may be explained by the action of the lymphatics of the udder in reabsorbing a part of the fat when the milk remains for a long time in the vessels of the udder.

There is also a considerable variation in the milk from day to day. This is usually not so great as between the morning and evening milking, but it not infrequently amounts to 1 per cent. Such daily variations may be ascribed to changes in the climate or other environment of the cows,the effect of storms, the effect of change of food, the effect of slight indispositions, ete.; but there are numerous conditions not usually or readily recognized by the owner that affect the composition of the milk. It has been noted in many instances that the normal effect of a slight febrile condition of the animal is to largely increase the percentage of fat and albumin. If the febrile condition continues, and particularly if it grows more severe, the fat then falls as quickly as it had risen, and to a correspondingly lower point. 
The variation in the percentage of fat in the milk first and last drawn is very great. The first milk drawn is much the poorer in fat. Differences so wide as 1 and 10 per cent of fat in the first and last few pints have not infrequently been noticed. This is due in large measure to the fact that the larger globules of fat, being of nearly the same size as the smaller milk ducts, pass along these vessels less readily than the more fluid portions of the milk, and are only drawn out with the last milk drawn. The milk first drawn has been in the milk cisterns and larger vessels for a considerable period of time, and so has been subject to the reabsorptive action of the lymphatics for a longer time, which also would tend to make it poorer in fat.

The food also has a considerable influence upon the quality of the milk, although the quantity of the milk is more easily affected by changes in the amount and character of the food than is quality. In fact, with cows kept under favorable conditions, with an abundant supply of food, it is hardly possible to increase the proportion of fat to other solids by a change in the food. On the other hand, while the amount of the various constituents of the milk is not easily affected by the food, the quality of the constituents themselves may be considerably influenced, notably in the case of the fat. Certain foods have a marked influence upon the character of the milk fat. Thus linseed meal, gluten meal and certain other foods make a soft, 
oily fat, while cotton-seed meal, the seeds of the various legumes and wheat bran make a hard fat. Constituents other than the fat are not so readily affected in this way.

Of the constituents of milk, the ash and the sugar are the least variable, the fat and albumin the most variable, while the casein usually bears a nearly constant ratio to the fat. The percentage of water also varies considerably. The causes of the variation of the fat have already been noticed. The proportion of albumin is very largely influenced by the physical condition of the cow, and it has been shown, notably by Van Slyke (see Chapter XIII.), that with what may be called normal milk, - that is, milk containing from 3 to 4.5 per cent of fat,- the proportion of casein rises or falls in almost exact ratio with the fat, but when the fat rises above this point the casein does not follow in the same proportion.

A notion is prevalent that the percentage of fat in the milk is also affected by the age of the cow; that during the first and second periods of lactation the young cow usually gives milk poorer in fat than when she is mature. During the years of greatest vigor the percentage of fat is supposed to be fairly uniform; but in cows of advanced age it may sometimes again fall to a low point. Recently some records have been published* that go to show that the age of the cow has little, if any, influence

*Holstein. Friesian Herd Book, vols. 13, 14, 15, 16, 17 . Cornell University Agr. Expt. Station, Bulletin No. 169. 
on the percentage of fat in the milk. In the one case the observations were made upon a large number of cows of all ages, for a week at a time, comparatively early in the period of lactation. In the other the observations were made upon a single herd extending over several years, and the percentages of fat are the average for the whole period of lactation The percentages of fat for the different ages are as follows :

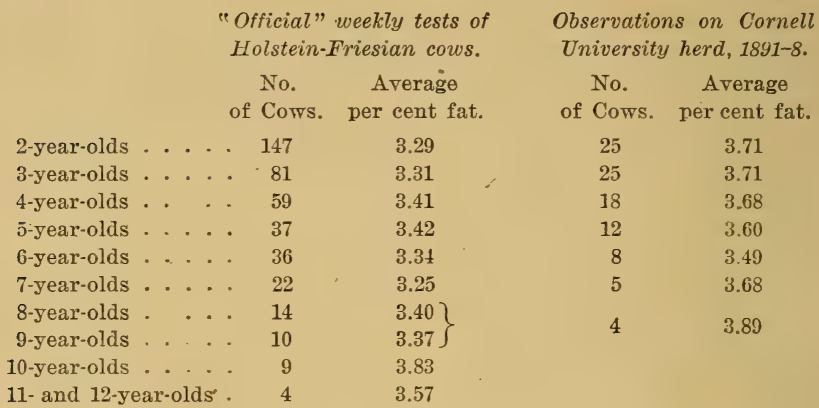

The breed of the cow also influences very largely the percentage of fat in the milk. Cows of certain breeds normally give milk much richer in fat than others.

The following, compiled from a large number of analyses made at various American Agricultural Experiment Stations, will give a general idea of the average composition of the milk of the more common breeds, so far as it relates to total solids and fat: 
Solids. . Fat.

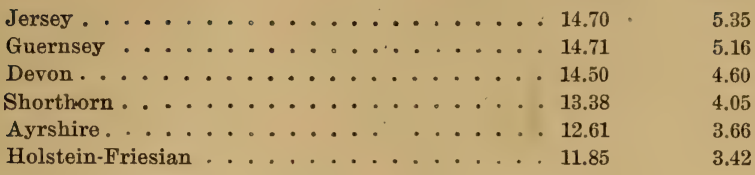

The variation due to breed includes not only the amount of fat, and the color and melting point of the fat, but the size of the milk globules. In some breeds the milk globules are uniformly large, in others extremely small, and in still others both large and small globules are found.

While there is a distinct difference in the quality of the milk of the different breeds, the different individuals in the breed also vary largely in the quality of the milk. The difference in the percentage of fat in milk from different cows of the same breed is quite as great as the average differences between the breeds; that is to say, the difference between the highest and lowest percentage of fat in the milk of different individuals of the same breed is as great as the difference between the average percentage of fat in the breeds giving the richest and poorest milk.

The variations due to the breed of the animal extend, of course, in some measure to the butter made from the milk. This is particularly true of the color and hardness of the fat. But while these differences are sufficient in amount to be characteristic, they scarcely affect the quality of the butter as a whole. While some partisans may 
contend that the butter made by their favorite breed is of superior quality, it would be well-nigh impossible, in any given case, by an examination of the butter, to say from what breed of cows it had been made. Butter of the very best quality in texture, color and flavor may be made from the milk of any breed of cows. 


\section{CHAPTER III}

THE PRODUCTION OF MILK-DAIRY CATTLE

THE prosperity of any dairy industry is very largely dependent upon the economic production of the raw material, namely, milk. Unless milk is produced at a profit to the farmer or dairyman, it is impossible to establish a permanently successful manufacturing industry : upon it.

While it is without the scope of this work to discuss all the phases of economical milk production, every manufacturer of milk products should have at least some understanding of the various conditions which so elosely underlie the prosperity of the industry.

Milk a maternal function.-As has been mentioned in a preceding chapter, milk is produced as a part of the maternal function of the females of the class mammalia, but while maternity is the efficient stimulus to milk production, the commercial production of milk in the highly civilized and specialized animal known as the dairy cow, is influenced to such a degree by so many other conditions that we are in danger of overlooking the part that maternity plays in milk secretion.

Wild animals, and, as a matter of fact, practically all domesticated animals, secrete milk solely to supply the young with food until such time as it is able to 
secure and digest food of the same kind and nature as its parents. The function of milk secretion in all such animals, then, begins when the young is born, increases rapidly for a few days or weeks, as the developing infant requires more food, and then gradually diminishes as the infant with continued growth begins to seek its natural and permanent food, and finally entirely ceases when the young is able to get its own subsistence, at the age of a few weeks in the case of most small animals, and in no event longer than a few months even with the largest forms.

The cow the only commercial milk producer.-History does not tell us how the cow came to be developed as the preëminent producer of milk for man's use. In all probability the milk of the goat and the ass was used by man before that of the cow. But in her development the cow has shown herself to be so much more adaptable to the commercial production of milk as to have distanced all other animals in this respect. There is no historical evidence that leads one to believe that in her wild state the cow had any greater tendency to give milk in excess of the demands of her offspring or for longer periods of time than many other animals. The domestication of the cow has resulted in developing an animal in which the capacity for secretion has been multiplied many times, and the duration of secretion has been made practically continuous. As a liberal estimate, a vigorous calf would not need more than 20 pounds of milk per day for the first four months of its life, or 2,400 pounds of milk, and this, or less, would be all that a 
normal wild or semi-wild cow would be likely to produce in a year. Numerous cows have lived that have produced more than ten times this amount, or 24,000 pounds of milk in a year, the largest amount on record being $30,318 \frac{1}{2}$ pounds of milk given by the Holstein cow, Pietertje 2d, in 1888.

Factors in the production of milk.-The chief factors on which the production of milk depends are: (1) Maternity, or the period of lactation; (2) the inherent capacity of the cow, which in general is largely determined by her breed and hereditary characteristics, but always to a greater or less extent independent of them; (3) the amount and character of the food supply.

The lactation period.-As has already been stated, the domesticated cow has been developed so that the production of milk is practically continuous. Nevertheless, pregnancy and parturition play a very important part in the production of milk. While cows may, and often do, secrete milk continuously for two, three or four years without producing a calf, on the other hand, the birth of a calf serves to stimulate the secretion of milk to such an extent that practically all dairymen are agreed that milk is produced under the most favorable conditions when the cow produces a calf at regular intervals each year, and this regardless of the value of the calf when born. It is also found that a period of rest before parturition is essential to the largest production of milk after the calf is born; that is to say, the cow will secrete more milk in a year if she goes dry for two 
to four weeks before calving, than though she is milked right up to calving, as often may be done. So far as can be ascertained, the good effect of this dry period is very largely physiological, the secreting glands in the udder are stimulated to greater activity by reason of having been inactive for a short space. While dairymen often speak of this period of dryness as a resting period, its good effect is probably not so much due to the resting of the general vital powers of the animal as to the physiological condition of the udder itself.

In most cows when the period of pregnancy has reached about the seventh month, there is a marked diminution in the flow of milk, and the udder shrinks rapidly in size. If now regular milking ceases, the cow soon "dries up," the udder shrinks away and becomes flaccid and empty, except for a small amount of watery saline fluid. About two weeks before parturition, the udder begins to take on renewed activity. It increases rapidly in size, but remains soft and spongy under normal conditions until a very short time before calving. If the cow is very fat, if she is fed heavily on stimulating, heating foods, or if there are other conditions that tend to plethora of the body, the swelling of the udder may be unduly large, take on an inflammatory character, and the udder becomes caked. In cows of a heavy milking habit this often is a very serious condition, and may even destroy the usefulness of the animal, or at least one or more quarters of the udder. It is obviated by taking care that the animal is fed only loosening and cooling 
foods, and that the bowels are kept free and open. Ordinarily, milk does not appear in the udder more than a few hours before parturition. It is usually abundant as soon as parturition takes place, and its regular and complete removal, having due regard for the condition of the animal, is a very efficient means of establishing a large flow. And if there are no troubles attending parturition and the establishment of the milk flow, the animal may be fed liberally as soon as the milk has lost its colostrum character, and under favorable conditions of health, food and treatment, the flow of milk will increase for two to four weeks after the calf is dropped, at which time it ordinarily has reached its maximum. From this time, favorable conditions continuing, the flow of milk may be kept very nearly constant for several months. If the cow is to produce a calf regularly each year, she will naturally be bred about three months after calving. It is not at all uncommon for cows to show a quite marked diminution in the milk flow immediately after service, but the best cows will continue to yield milk with very little diminution until the foetus begins to grow and make demands upon the mother at the fifth or sixth month of pregnancy. From this time until the seventh month there will be a regular and quite rapid diminution in the flow until the tendency to go dry is strong, as has already been said, at about the seventh month.

The operation of milking.-The production of milk is also dependent to a considerable extent upon the operation of milking. A skilful milker will get ap- 
preciably more milk from a cow, or lot of cows, in the course of a season than an unskilful one. The milk should be drawn from the cow as rapidly, as quietly, and as easily as possible. It goes without saying that it should also be completely removed. Downward stroking manipulations of the udder are of some use in removing the very last portions, but the method advocated by Hegelund $*$ for this purpose some years ago has not been generally adopted. Regularity in the time of milking is also an important factor in securing large amounts, more particularly in keeping up the milk flow, and preventing rapid drying off toward the close of lactation. The interval between milkings also affects the amount produced. Up to a certain limit the amount of milk produced will be increased by shortening the milking period, and it is by no means infrequent to milk cows three or four times daily, at intervals of eight or six hours, and practically all large records of production are made under such treatment. When the interval is shortened to less than six hours, the disturbance to the animal checks the milk flow quite as much as the increased frequency of removal tends to increase it, and no advantage has as yet been gained by milking cows oftener than four times a day. It is difficult to make an exact standard with respect to the relation between frequency of milking and amount of milk secured, but it may be said in general that the amount of milk will be increased if the cow is milked as often as her udder becomes moderately distended.

*Cornell University Agricultural Experiment Station Bulletin No. 213. 
Cows easily contract habits with respect to milking, and often these habits are very disagreeable and amount to a good deal of loss. Many cows will not "give down" the milk unless they are fed at, or just before, the time of milking. Occasional cows will yield very much more milk for a favorite milker than for a stranger. For this reason it is a common practice, particularly in small herds, that each milker should milk the same cows each day. In large herds, however, where milkers necessarily have to be changed frequently, pains are taken to prevent the contraction of any such habits, and the cows are milked indiscriminately, and so have no chance to form an attachment for any particular milker. It is a common opinion among dairymen that milking habits are more easily formed during the first lactation of the heifer, and care is taken that the heifers be milked as well as possible, and that their lactation period be prolonged as closely as possible up to the time of dropping the second calf. There is no disadvantage in such practice, even if it often fails to yield tangible results. Mechanical milking machines, that have been the subject of so much and so long continued experiment, have now reached practical form, and are being successfully introduced in many large dairies.

The individual capacity of the cow.-No single fact in milk production is of more importance, so far as profit and loss is concerned, than that the cow is a law unto herself in respect to the amount of milk that she can be made to give. Profitable dairying 
depends upon distinguishing between productive and non-productive cows, and there is no one thing that will secure greater improvement to a dairyman than weeding out the unprofitable cows in the dairy, and supplying their places with those that are profitable producers; at the same time, there is no factor more generally neglected by the dairymen of the United States than this. It therefore becomes a matter of some importance that the dairyman should be skilled in distinguishing between productive and unproductive cows.

Relation of form to capacity.-In the development of the dairy cow, and particularly in the formation of the various dairy breeds, it has been noticed that the capacity to produce milk is to a certain extent correlated with certain well marked and easily recognized characteristics of form. This has led to the distinction between the so called dairy and beef types of animals. The chief characteristic of the dairy form is the wedge shape; that is, the larger development of the hind quarters, and the corresponding less development of the fore quarters, so that if the cow is viewed from the front or side, there is a distinctly wedge-shaped appearance, with the apex of the wedge toward the head. This is contrasted with the characteristic rectangular shape of the beef animal. In connection with the wedge shape, a large degree of angularity and lack of muscular development, particularly along the ribs and loins and on the shoulders and thighs, is quite as characteristic of the dairy animal as the wedge shape itself. In addition it is, 
of course, essential that the cow, in order to be a profitable milk producer, must have a large, capacious udder, full of secreting follicles, and, as the udder is an external organ, its size and capacity are quite readily and easily determined by examination. Capacious digestive and respiratory organs are also important, as indicating activity of the vital functions of the animal, and strong, vigorous constitutional powers. So useful are these external characters known to be as indicating capacity for secretion that dairymen find it worth while to train themselves in recognizing these indications and comparing cows one with another with respect to their external indications for capacity for milk secretion, and a large amount of instruction in schools and colleges is given along these lines. Training in these matters is facilitated by the use of score cards or scales of points, which enumerate and describe the general external characteristics of the cow, and assign numerical values to the different characters in proportion to their assumed relative importance. Most breeders' associations, and very many colleges, have formulated and used such scales of points. They naturally vary more or less, according to the ideas of the persons who formulate them. A sample eard is shown on the next page. The chief value of a score card is to teach the novice to make a careful examination of every part. His final judgment, however, should be based on the individual as a whole, and this is best trained by practice in comparing individuals side by side. 


\section{Score Card for Datry Cattle}

Scale of Points-Cow

General Appearance-

Weight. - Estimated....... pounds; actual.

Form-Wedge-shaped as viewed from front side and top...

Form.- - Spare, as indicated by prominent joints and clean bone and lack of muscular development along ribs and

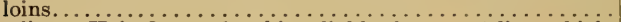

Quality.-Hair fine, soft; skin pliable, loose, medium thickness; secretion yellow, abundant...................

Constitution.-Vigorous, as indicated by alert expression, evidently active vital functions, and general healthy

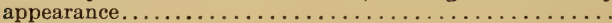

HEAD AND NECK-

Muzzle-Clean cut; mouth large; nostrils large..........

Eyes.-Large, bright

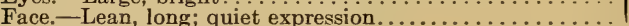

Forehead.-Broad, slightly dished.

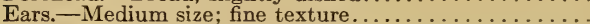

Neck.-Fine, medium length; throat clean; light dewlap..

\section{Fore- AND Hind-QUarters-}

Withers.-Lean, thin. Shoulders.-Angular, not fleshy .. .

Hips.-Far apart; not lower than spine....।

Rump.-Long, wide, comparatively level... $\}$

Thighs.-Thin, long.

Legs. - Straight, short; shank fine $\ldots \ldots \ldots \ldots \ldots \ldots \ldots$

\section{BoDY-}

Chest.-Deep; with arge girth and broad on floor of chest

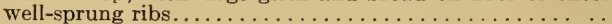

Abdomen.-Large, deep; indicative of capacity; well sup-

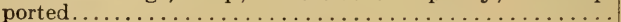

Back.-Lean, straight, chine open. Tail.-Long, slim, with

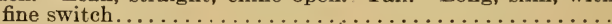

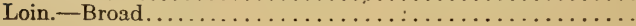

Milk-secreting Organs-

Udder.-Large, long, attached high and full behind; extending far in front and full; quarters even................

Udder.-Capacious, flexible, with loose, pliable skin covered

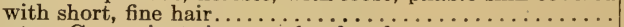

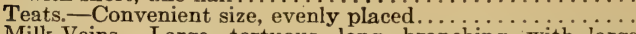

Milk Veins.-Large, tortuous, long, branching, with large milk wells. Escutcheon.-Spreading over thighs, ex-

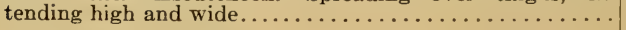

\section{Total}

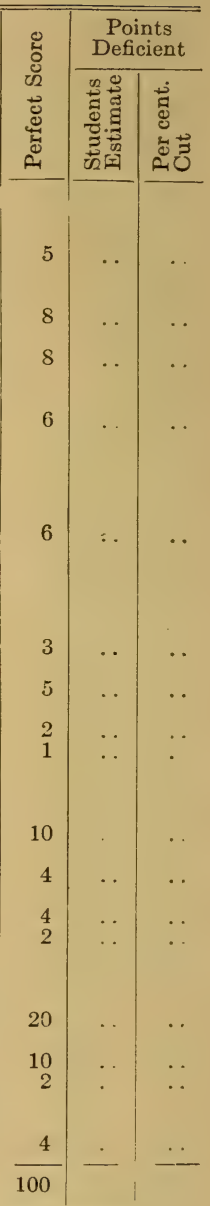

Animal

Date

Seored by

Total Score 
Through natural aptitude, through careful and systematic training, and through constant practice, very many persons become expert in the selection of cows, and they are able to distinguish with a good degree of accuracy between cows that are large or small producers. Yet no matter how much skill may be attained along these lines, the fact still remains that the external conformation fails to coincide with the actual production of the animal in a sufficient number of instances so that it is never safe to depend entirely upon outward indications in selecting cows.

Value of records of production.-The statement is frequently made, and generally accepted, that there is no means of determining the prospective value of a cow for the production of milk that can compare with a knowledge of what the animal has already done as determined by an actual record of production in both milk and fat extending through a year or a complete period of lactation. This is so well recognized that all dairy cow breeders' associations are making provision for the segregation of the large producing animals of the various breeds into a class by themselves, known as the advanced registry or register of merit, admission to which is gained only by actual production, authenticated by. disinterested supervision, and breeders of dairy cattle are expending thousands of dollars each year to secure such authenticated records of their animals.

Necessity for keeping records.-If records of production are recognized by breeders ot pure-bred animals as an essential factor in the breeding and 
selection of their animals, they are no less useful to him who keeps cows merely for the production of milk, without regard to the productive value of their posterity. It has been demonstrated many times that in milk-producing herds where no records are kept there will be found anywhere from one quarter to onehalf, and sometimes more, cows whose total production is insufficient to pay for their feed and care, such cows being kept at an actual loss to their owner, and their deficiency covered up by the higher producing animals. There are many herds, the total production of which may be profitable to the owner, in which from 10 to 25 per cent of the individual cows are kept at a loss. The great reason for this condition of affairs is the fact that even a careful observer will fail to discriminate between a profitable and unprofitable animal, unless an actual record of the production of each animal is kept from day to day. This is comparatively seldom done, especially in herds maintained solely for milk production. But such records may be kept at comparatively little cost, even when the butter fat is determined, and their cost will be returned many times over to the owner if he acts upon the results of the records, and discards from his herd those that are shown to be unprofitable. As has already been stated, there is no one thing which wonld result in more increase to the prosperity of the dairy industry as a whole, and more profit to the individual owner, than the general keeping of records of production, and the weeding out of the unprofitable animals, as shown by such records. . 
Where the keeping of records of production is likely to prove burdensome on individual small owners, it has been found that this work may be done to great advantage coöperatively, and the success of cow-testing associations in many localities attests this fact.

Food as a factor in milk production. - It is selfevident that feed must be an important factor in the production of milk, since the solids in milk are produced directly from the food. It matters little what the conformation or the hereditary powers of the animal may be, she cannot produce milk in large amounts for long periods of time unless she is abundantly supplied with the material from which the milk is made, and, in general, that cow is the best and most economical that can transform the largest amount of food into a corresponding amount of milk. The subject of feeding of domestic animals, and particularly the dairy cow, has received a great deal of study and investigation in this country and Europe in the last fifty years, and very notable advances have been made with respect to the science and art of feeding animals, and a large literature has sprung up in regard to this question. This work is not intended to be a manual of cattle feeding in any sense, so only the most general principles in respect to feeding dairy cattle will be touched upon. The ideal ration for the dairy cow must be abundant; it must be nutritious; it must be palatable; it must be succulent; it must provide a sufficient balance between the proteid and non-proteid nutrients, and it must be cheap. 
A great deal has been said and written about the amount of food that should be given to a cow in milk, and various standards have been established. These standards are useful to guide the inexperienced feeder, the chief difficulty concerning them being that one is likely to get the idea that if the standard is scientifically established on a proper basis all that is necessary to do is to administer the standard amount of food to the animal, and a given result will be obtained. Such is not the case. Animals vary in the amount of food that they are able to use, and more particularly in the amount that they can economically turn into product. In respect to the dairy cow, three things will happen if she is fed continuously all the food that she will eat regularly without disarranging the digestive organs or going "off feed:" (1) She will secrete a certain amount of product (milk and milk fat), and at the same time gain in weight, or will put fat on her body. (2) She will make a similar amount of product, but will make no gain in weight, some of the food apparently going to waste. (3) She will use all of the food consumed for the production of milk, and will increase regularly in milk secretion as the food is increased up to the limit of her capacity to eat and digest. It is needless to say that the cows in this latter class are the most valuable to their owners, and experience has determined that they are more numerous than was formerly supposed.

The ideal ration.-A liberal and economical ration for the best type of dairy cow is all the roughage 
(hay, silage, roots, ete.) she will eat, and one pound of grain food (concentrates) for each three to four pounds of milk she produces; and in many cases this will be found to be just about all she will eat regularly without going "off feed."

A second consideration in an ideal ration is that it should be nutritious; that is to say, there should be a certain relation between the digestible and indigestible parts of the food. The cow is a ruminant, and the digestive organs of ruminants have been developed to use comparatively large amounts of foods, relatively small portions of which are digestible. On the other hand, it is possible so to combine the ration that it will be so bulky that the digestible portion will be insufficient to support the animal and provide a maximum amount of product. The ration should be sufficiently bulky, on the one hand, to fully distend the stomach and other digestive organs. At the same time, there should be enough digestible material to fully meet the requirements of the animal. If, however, we go to the other extreme and make the ration of too concentrated or easily digested foods, the animal will have a superabundance of digestible material in too small a bulk to properly distend the digestive organs. The most frequent result of this is that the appetite becomes cloyed, the digestive organs disarranged, and the animal goes "off feed." Practical experience has shown that a proper balance is reached when about two-thirds of the total dry matter of the ration is in the form of roughage, and one-third in the form of concentrates. 
It goes without saying that a ration cannot be of the highest degree of effectiveness if it is not palatable, if the animal does not eat it, not only readily but eagerly. The factors of palatability are not well understood, nor always easily recognized, and our knowledge of them is to a very considerable extent empirical, and the result of actual observation and experience. About all that can be said in this respect is that of two rations or combinations of food similar in all other respects, that one will be most effective that is most readily eaten by the animal. There are certain adventitious aids to palatability, such as salt, water or succulence, and freshness. The peculiar characteristics of certain plants also make them particularly palatable or unpalatable for certain animals, or classes of animals, and in addition there are various vegetable aromatics and semi-tonics, and certain inorganic salts, that are recognized as having a marked effect upon the appetite. A continuous use of these latter for healthy animals seldom results in distinct advantage. The secretion of milk seems to be intimately connected with the water content of the food. Milk itself is a watery substance (ordinarily about seven-eighths water), and of course the water, which makes up so large a part of it, demands a corresponding consumption of water by the animal. It seems almost necessary that a certain part of this water should be regularly incorporated with the food or, in other words, it is of great advantage for the secretion of milk that at least a part of the food should be composed of materials containing large 
amounts of water, like fresh forage, silage, fruits, roots, etc. So well is this recognized that many dairymen find it economical to provide a regular supply of succulent foods for their cows in milk, even though the nutrients in such foods cost more than similar amounts would in the dry state.

In all the studies and investigations that have been made with respect to the nutrition of domestic animals, no one has received more attention than the relation of the proteid, or nitrogenous part of the nutrients, to the non-protein part. Disregarding the protein supply so far as the maintenance of the animal is concerned (and a certain amount of protein, as is well known, is requisite to maintain life), there are considerable amounts of protein in the milk, the most important being the casein and albumen of the milk, which together comprises more than 3 per cent of the milk or about 25 per cent of the milk solids. This protein, of course, must come from the protein supply in the food. The problem of furnishing sufficient protein in dairy foods has been greatly simplified by the introduction of certain byproduct commercial foods that are now abundant on the markets, so that it is no longer difficult, nor very expensive, to provide a sufficient amount of protein, and it is found that it is not necessary to "balance" the proteid and non-proteid nutrients so carefully as formerly. This balance is ordinarily expressed by the nutritive ratio, and a dairy ration is now considered at least fairly satisfactory if the nutritive ratio falls anywhere between $1: 4.5$ and $1: 6.5$. 
Last of all, the ideal ration should be cheap, not necessarily in the sense of being made up of low grade or low cost foods, but from the standpoint of furnishing the largest possible amount of digestible nutrients at the lowest cost. Home-grown foods ordinarily cost the dairyman less than commercial foods, and the milk producer will ordinarily feed most economically who uses the largest possible amount of bome-grown foods. On the other hand, commercial foods in nearly all markets, if well selected and carefully purchased, may be fed at a profit if the cows are well selected and productive. Market fluctuations in various localities make it possible for the consumer of commercial foods to effect considerable saving in his feed bills, and the dairyman can scarcely be called an intelligent one that does not keep well-informed in regard to local market conditions and prices, with respect to commercial foods, and vary his purchases accordingly.

Selection of breed.-The development of the milking powers of the dairy cow has been the result of evolution and selection: So far as is known, all of the breeds of dairy cattle have been brought up to their present capacity for production by constantly selecting the highest producing individuals, and raising the offspring from these on both sides; that is to say, the selection of the bull from a high-producing cow has been considered quite as important as the raising of female calves of such cows, in securing improvement. The importance of a high-producing animal has already been discussed. In order to produce such 
animals, constant care must be used in the selection and breeding.

In the formation of a dairy herd one of the first questions to be considered is the choice of a breed, and whether the herd shall be made up of pure-bred or grade animals. There are several well-known breeds of dairy eattle in the United States, each having distinct qualities, and each having strong partizans. While, undoubtedly, some breeds are better fitted for certain conditions of soil and climate than are others, still the matter of the choice of a breed may well be left to individual preference. In almost any location one may choose a breed for which he has a strong liking, either real or fancied, and be assured that he can establish with it a successful herd. Jerseys, Guernseys, Holsteins, Ayrshires, Swiss, Devons, Red Polls, and even Shorthorns, make a varied and fairly long list of breeds from which to select.

Pure breds and grades. - Whether purely bred or grade animals should be selected is a matter upon which there may be more difference of opinion. By purely bred animals are meant those that are recorded in the herd books of their respective breeds. This is the narrow, restricted sense of the term "pure bred," but it is the one in common use in this country. A grade animal is the offspring of a pure-bred sire and a common or grade dam. The offspring of a purebred sire and a common or "scrub" dam, is called a half-blond; the offspring of a pure-bred sire and a half-blood dam is called a three-quarter-blood; a three-quarter-blood in turn bred to a pure-bred sire 
will produce a seven-eighths-blood, and so on ad infinitum. Since only the offspring of pure-bred parents on both sides are eligible to registry in the herd books of any of our dairy breeds, it follows that no matter how far the process of grading up by the use of pure-bred sires on grade females is carried, pure breds, in the technical sense, can never be produced. Since pure-bred animals are sought after for breeding purposes, the offspring of pure-bred animals are, therefore, more valuable, and command a higher price than do the offspring of grades. Hence, if the value of the calves is taken into consideration, a purebred herd is a more productive one, other things being equal, than a grade herd. On the other hand, by the grading-up process above referred to, continually selecting the cows that are the best producers, it is entirely possible, and by no means difficult, to establish a herd of grade.cows that will equal, in the production of milk, any herd of pure-bred animals. Hence, if the main product alone is sought after, a grade herd may produce as largely as a pure-bred one; but if the by-product in calves is to be taken into consideration, a pure-bred herd certainly has an advantage over a grade herd of equal productive capacity.

Maintenance of the dairy herd.-A dairy herd may be maintained in two ways: First, by continued purchase of mature animals to replace those whose period of usefulness has passed. There are many conditions under which this may be a wise practice. If it is desirable to have the whole herd composed of cows 
in their full productive eapacity; if there is abundant opportunity for selection and purchase near at hand; if there is a reasonably good market for cows that are undesirable, and if one has reasonable skill in selecting, and good ability in bargaining, a herd of highproductive capacity may be more easily and more cheaply maintained in this way than by attempting to raise young animals to replace those that are worn out.

The other method of maintaining the herd is, of coirrse, by raising calves to supply the place of old cows that are no longer profitable. Such a herd will always contain a considerable number of young animals that have not yet reached full development, and, therefore, such a herd will seldom equal in average production per animal a herd that is maintained wholly by purchase. At the same time, a greater degree of uniformity of type may be maintained where the animals are raised. If land is abundant and cheap, the cost of raising a calf, up to the time that she becomes a fully developed cow, will be less than that of purchasing a similar animal outright. Through force of circumstances by far the greater number of dairymen must rely on raising the calves necessary to maintain the herd. This being the case, the ordinary dairyman will need to provide himself with the services of a bull suitable to produce useful dairy cows. In most cases he will need to own this bull, so that the question of the selection and care of the breeding bull has an important bearing upon the maintenance of the dairy herd.

Selection 'of the bull.-It goes withont question that 
such a bull should be purely bred. There are still far too many immature and ill-bred bulls in use. As a matter of convenience, a yearling bull is turned with the herd about the first of June, and in the course of two or three months, when all, or nearly all, of the cows have become pregnant, and he has fattened up, he is sold again at a price little, if any, below his cost, and the dairyman is without the trouble of the care of a bull for several months. If, in addition to this, little attention is paid to the breeding of the bull, it is easily seen that little, if any, improvement in the character of the herd can be expected from his offspring. The progeny that a bull has already produced, is by far the best index of his usefulness that a prospective purchaser can have. Cattle do not reach full maturity, in either sex, until they are about four or five years old. Consequently, the best bull to select is a bull not less than four years old that has already begot cows of a high productive capacity. Such a bull is, of course, more difficult to control, and more expensive to keep, but he is worth many times the trouble and expense, as compared with an immature yearling.

In the selection of a bull much has been made of various so-called milk signs: Rudimentary teats, milk veins, escutcheon, etc. While each and all of these may be of some value, they are by no means to be set against the record that a bull has made in the production of his daughters. Other personal characteristics of the bull that indicate vigor of constitution, good digestive organs, and vital activities, in general, 
are a loose, mellow hide; a bold, bright eye; an active gait and disposition. These are of great importance as indicating capacity to reproduce similar characteristics in his offspring.

Management of the bull.-The management of the bull on a dairy farm is often a matter of a good deal of trouble and perplexity. In a herd of ordinary size a single bull is sufficient, and it is usually necessary that he be kept from the herd during the greater part of the year. This means that he must be kept in solitary confinement. The result of this is, too often, that his temper becomes uncertain, and his breeding powers impaired from lack of exercise. In all cases where it is possible to do so, it is better that the bull should run with the herd of cows. With a little care this can frequently be done, particularly during the summer and fall, when the cows are all pregnant in a spring calving herd, and in the spring and early summer, when the cows are all pregnant in a fall calving herd.

The powers and temper of a bull may also be safeguarded by giving him exercise on a tread-power, or hitched by a long rope or chain to a wire between two upright posts, or attached to a pole balanced upon a post so that he may move around it. Occasionally, also, the labor of a bull may be utilized upon a tread-power for cutting feed, pumping water, or separating milk. Sometimes a particularly handy owner will break a bull to work to harness or in a cart. It may even be time and labor well spent to give a bull walking exercise. A good, vigorous ani- 
mal, carefully kept, should retain his breeding powers up to eight or nine years of age, or even beyond.

Grading up the herd.-The successful and progressive dairyman will not only give his best efforts toward securing a herd that will make a satisfactory production, but will look to the future, and secure still further improvement by breeding from his herd succeeding generations that shall be even larger producers than their ancestors. Such a dairyman may, if he chooses, secure as the foundation herd pure-bred animals that may be depended upon to transmit their qualities to their descendants. But with even the highest-bred animals there will be the necessity for selection, if the original standards of production are to be maintained, to say nothing of being increased. On the other hand, the large majority of dairymen seeking to improve their herds must depend, more or less, upon the individual animals they have already on hand as the basis from which to start the improvement. In either case, careful selection must be practiced, and a knowledge of at least the elementary principles of selection is necessary for progress along this line. It is proposed, then, briefly to indicate the lines along which an attempt to breed up, or improve, a herd of common, native or mixed cattle is most likely to prove successful.

In the improvement of a herd of cows it has been very common to recommend that the practice should be to use a pure-bred bull, and to raise the heifer calves from the best cows in the herd. Whether or not this practice is correct will depend, to a great 
extent, on what is meant by the term "best cows." If it means merely that the heifer calves from the common cows that are the highest producers are to be raised, it cannot be accepted without qualification. The first and great step in improvement under such a course of breeding comes from the prepotent qualities of the bull. Logically, therefore, the best calves to raise are those in which the prepotent qualities of the male are most clearly shown at time of birth, such as color markings, or similar characteristics. These may or may not be the offspring of the highest-producing cows. The foundation herd of cows is admittedly inferior, even in the best of them. If proper judgment has been exercised in selecting the bull, there is in him greater chance for improvement than exists in any of the cows, even the best. It would, therefore, seem to be good logic and safe practice to disregard the milking qualities of the cow entirely in the first generation, and depend on the prepotency of the bull.

A great advantage in the rapid improvement of a herd of cows under this grading-up process is the ability to raise a large number of individuals up to the time they begin to produce, so that a greater number may be available from which to select. In the first generation, therefore, it is a great advantage to be able to raise all of the half-blood heifer calves that resemble their sire and that are born strong and with good vital powers, irrespective of the qualities of their dams. In many cases, however, the circumstances of the owner do not admit of rearing so large a number, and some selection must be made at the time of birth. 
If we concede that it is not an entirely safe practice to base this selection upon the producing capacity of the dams, we must look for some other basis of selection.

It is a generally accepted principle of heredity that an animal which shows a tendency toward variation is one that is not likely to be prepotent. We desire to raise calves from cows that are not prepotent, in order that the prepotent qualities of the bull may have full scope. Cows, then, that show tendency toward variation, particularly toward improvement, are logically the ones over which the male is most likely to be prepotent, and at the same time the calves show the greatest tendency toward improvement. It is comparatively easy to determine in any herd of cows those which show the greatest tendency toward variation, and more particularly those which show the greatest tendency toward improvement when their conditions are made more favorable. We can then determine the cows from which we are likely to get the best half-blood heifer calves by giving the herd more and better feed, and selecting calves from those animals which show the greatest improvement under such a change of treatment.

Experience has shown that where the principles stated above have been carefully carried out a very satisfactory improvement has been secured in the first generation. It is not uncommon to find an increase of fully 50 per cent in the average production of halfblood cows over their common mothers. Experience has also shown that in the second generation the three-quarter-bloods are not nearly so uniform as 
the half-bloods, and frequently show little, if any, increase in average production, though a few individuals will show a marked improvement. The question then comes as to how to secure a greater uniformity and a higher average production in the second generation or the three-quarter-bloods.

If the selection of the original pure-bred bull has been a wise one, and if he was a young animal at the time of his purchase, there will be a considerable number of his half-blood offspring ready to be bred while he is still in the zenith of his powers. Most breeders hesitate to breed such an animal to his own offspring, and it is seldom recommended. But if inbreeding is ever likely to be followed with useful results, it will be under just such conditions; and, in proportion as both the bull and the half-blood heifers show strong individual vital powers, the practice is to be recommended. In a majority of cases the very best bull to breed to a lot of high-quality, uniform, half-blood heifers is their own sire, if it is desired to secure greater uniformity and greater average production in their offspring. The reason for the lack of uniformity in the three-quarter-bred offspring is the fact that reversions occur to the qualities of their common and mixed grandmothers. It will require, then, even stronger prepotency to overcome this tendency to reversion, and the animal which is most likely to be prepotent over such half-bloods is their own sire.

It must be remembered that the improved production in the first place was secured by improving the 
conditions of environment, which, so far as the ordinary dairy herd is concerned, means simply more food; and that it was perpetuated in the case of purebred animals by selection. Having obtained improvement now by the use of a pure-bred male on common or mixed females, it is, of course, necessary that it shall be maintained by liberal care and feeding. A pure-bred animal can transmit only its inherited tendencies, and if these inherited tendencies are not backed up by abundant and nutritious food, the improvement secured is sure to be lost. Having now secured a marked improvement in two or three generations by the use of a pure-bred male on common or mixed females, with intelligent selection and intelligent inbreeding, the further course of improvement is the maintenance of proper conditions of environment and careful selection. As generations come on, characteristics of the original pure-bred sire will become more and more fixed and uniform, reversions will be less and less frequent, and the herd will be practically pure-bred from the standpoint of the capacity of individual members to reproduce their characteristics, though they never become eligible to registration in the herd book. This is a course that has been successfully practiced in the improvement of a large number of herds of dairy animals, and is entirely within the reach of anyone of ordinary skill and intelligence.

Major and minor dairy breeds.-The breeds of dairy cattle have been developed in the main in localities where, for one reason or another, a dairy industry 
has sprung up, and where the localities have been more or less isolated, and the cattle have developed from the local cattle of the district by constant selection of those. that are the most plofitable producers. In some cases this selection has been going on for more than a hundred years. While the domestic ox is more or less variable in size, in conformation and in color, the number of breeds of cattle is not large, and they group themselves into the dairy and beef breeds, according to the purpose for which each has been selected.

At the present time in the United States there are about twenty well-recognized breeds, in about half of which the dairy is the leading characteristic. Four breeds maintain a leading position among dairy cattle; namely, Jersey, Guernsey, Holstein-Friesian and Ayrshire. Five other breeds occupy a minor position, but are still numerous enough to demand attention; namely, Shorthorn, Red Polled, Brown Swiss, Dutch Belted and Devon. Four breeds are native of Great Britain; namely, Ayrshire, Shorthorn, Red Polled and Devon. Two, Jersey and Guernsey, are native of the Channel Islands. Two, Holstein-Friesian and Dutch Belted, are native of the Kingdom of the Netherlands; and one, Brown Swiss, comes from Switzerland.

The breeds are easily distinguished one from another by size, conformation and color markings. They all have the characteristic wedge-shaped form of the dairy animal distinctly developed, and all have noteworthy dairy capacity, as shown by their large, symmetrical udders. 
Jerseys.-Jersey cattle, as the name indicates, originated upon the Island of Jersey, which is the largest

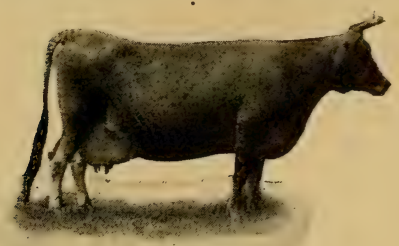

Fig. 2. Jersey cow. one of the Channel Island group, a group of about twenty small islands lying in the English Channel, near the coast of France, but belonging, politically, to Great Britain. There is no doubt that the cattle originally upon the island came from the mainland of France. For more than one hundred years the people of the Island of Jersey have absolutely prohibited the importation of live neat cattle from any other country, and the blood has therefore been maintained pure for more than one hundred years. Somewhat before the middle of the nineteenth century, dairying began to be an important farm industry upon the island, and the farmers began to give attention to the development of their cattle, both as to production and to form, and the development has been continuous ever since.

Jersey cattle are characterized by small size, spare, angular forms and curved outlines, dished faces, crooked legs, and often rather crooked backs. They

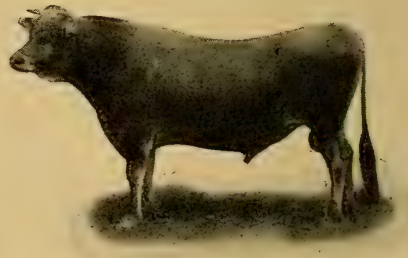

Fig. 3. Jersey bull. have a rather delicate, nervous organization, and, when carefully handled, are extremely docile and 
gentle, but they are easily disturbed by ill usage, and under such unfavorable conditions, the males particularly, often become ill-tempered and unmanageable. In color, they are fawn, shaded through the various shades of gray to black, often more or less spotted with white. The tips of the horns, muzzle, tongue and hoofs are black. In England and in the United States, Jersey cattle with no white markings have always been favorites, and this has had a considerable influence upon breeders upon the Island of Jersey, but there still remain a considerable proportion of animals more or less spotted with white.

Jersey cattle yield moderate amounts of milk rich in butter fat, the percentage of fat running ordinarily from 4.5 to 6 in cows in full flow of milk. The fat globules are also large and highly colored, giving the butter a very attractive appearance.

The first Jerseys were introduced into the United States about 1850, though they increased slowly until about 1870 , since which time they have increased rapidly, and have adapted themselves to dairy conditions over the whole country from Canada to the Gulf of Mexico, and from Maine to the Pacific Coast. They are the most widely distributed and the most numerous of any of the dairy breeds.

Guernseys.-Guernsey cattle originated upon the Island of Guernsey, another island of the Channel Island group, and next in size and importance to Jersey. The conditions under which Guernsey cattle developed are almost identical with those upon the Island of Jersey, and the same regulations with 
respect to importations from outside have also been enforced. Guernsey cattle resemble Jerseys in many respects, particularly as to amount, quality and color of the milk and milk fat, and they undoubtedly had a common origin with Jerseys on the mainland of France. They are distinguished from the Jerseys by slightly larger size, by rather straighter forms, and by a distinctly yellower color of the skin. In color they are uniformly fawn and white. Occasionally animals are seen that are brindled, but they are not common. The muzzle and the hoofs are flesh-or amber-colored, instead of black, as in the case of the Jersey.

Another island of the Channel group called Alderney supports cattle. The Island of Alderney is a part of the Bailiwick of Guernsey, and the cattle on

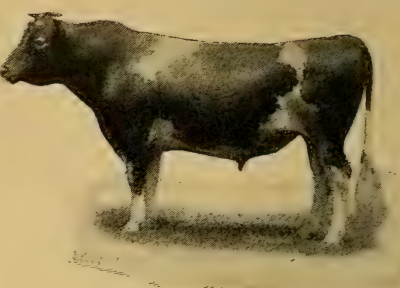

Fig. 4. Guernsey bull.

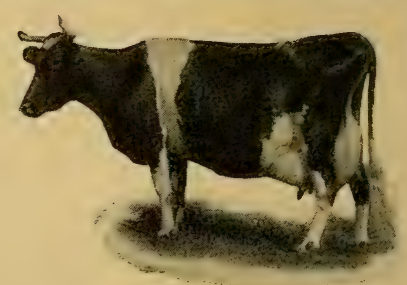

Fig. 5. Guernsey corr. Alderney are in no sense distinct from those on Guernsey, and at the present time are all included in the same breed.

Holstein-Friesians.-Holstein-Friesian cattle or, as they are more commonly called, Holsteins, are natives of the Kingdom of the Netherlands, and originated in two provinces of that kingdom, namely, North Hol- 
land and West Friesland. The name Holstein is an entire misnomer to this breed of cattle, so far as any geographical distinction is concerned. None of the ancestors of the cattle of this breed ever came from the Duchy of SchleswigHolstein, although the cattle of these districts, and some other Prusșian provinceś, are not greatly different

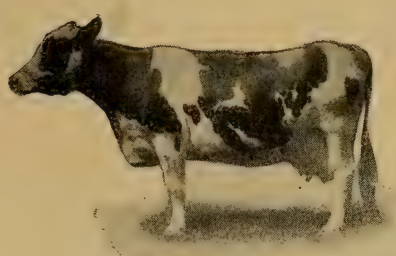

Fig. 6. Holstein-Friesian cow. from what are known in the United States as Holstein cattle. The proper geographical name for this breed would undoubtedly be Dutch. Dutch farmers have long been noted for their thrift, and the manufacture of dairy products, particularly of cheese, has been an important industry in North Holland for very many years. The land is fertile and eminently suited to the production of grass, but it is high priced, since it is pro-

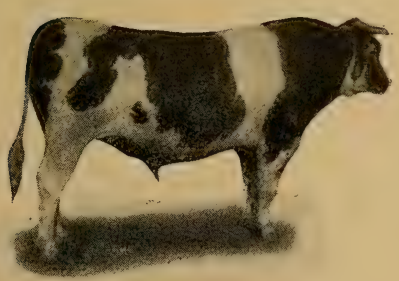

Fig. 7. Holstein-Friesian bull. tected from the encroachment of the sea by an extensive series of costly dykes and embankments.

In order that the Dutch farmer may make dairy products profitable on such high-priced land, he must have a very efficient animal, and under these circumstances the Holstein cow has been developed. The natural conservatism of the 
Dutch race has prevented the importation of animals from other countries, and so the race has been kept practically pure, some authorities say for as much as two hundred and fifty years. The Dutch cattle have been developed very largely upon grass in luxuriant pastures in the summer time, and on hay, supplemented with very little grain, in the winter. This has resulted in developing an animal of large size, capable of yielding a large flow of milk, but milk not very rich in fat; which is the prominent characteristic of this race of cattle.

Holstein cattle are large in size, ranking well up with the Shorthorn, Hereford and other beef breeds. They are inclined to have straight bones, long faces, straight, sometimes rather long legs, and straight backs. Many animals, however, have a distinct droop to the rump from the hip to the root of the tail, which breeders constantly select against.

In color, Holstein eattle are black and white, and in any proportion, running from almost pure white to almost pure black, and, in cases where the colors are more equally distributed, the colors may be finely broken up, or may be in large patches. Most American breeders prefer an animal rather more than half white, with the black and white colors in rather large areas. Peculiarities of coloring, either in proportions or markings, do not run very strongly in family lines, and an animal that is largely white may, and often does, produce offspring in which black is the predominating color, and vice versa.

Holstein cattle were introduced into the United 
States about 1860 . They increased very slowly until about 1880, since which time they have become very numerous, particularly in those localities where the sale of milk for market purposes is the chief dairy industry, and in many such localities they are rapidly coming to be almost the only dairy breed kept.

Ayrshires.-Ayrshire cattle, as the name implies, originated in the county of Ayr in southwestern Scotland. Up to about 1800 there was no particular type of eattle in this locality, but about this time dairying began to be developed, particularly in the parish of Dunlop, and the more enterprising dairymen began to select cows for dairy production from the common stock of the country. It is supposed that the original stock of the county of Ayr had a more or less mixed origin, but the Ayrshire breed took on distinctive characters as early as 1830, and since that time has developed into one of the chief dairy breeds in Scotland, in Canada and in the United States.

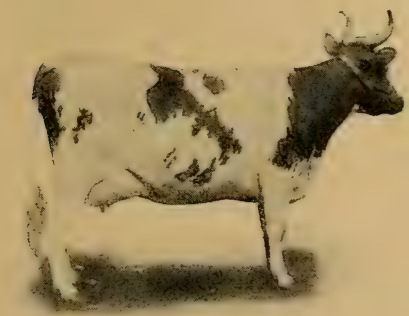

Fig. 8. Ayrshire cow.

Ayrshire cattle are of medium size, distinctly larger than the Jersey, and distinctly smaller than the Holstein. They are characterized by extremely straight and smooth forms, with round bodies, slim necks and legs, and long, slim, upright horns. They are extremely active in disposition, and alert, and stylish in appearance. These qualities have been developed 
as fancy points by many Ayrshire breeders. Their activity and disposition leads them to be extremely good grazers, and they are better suited to sparse and

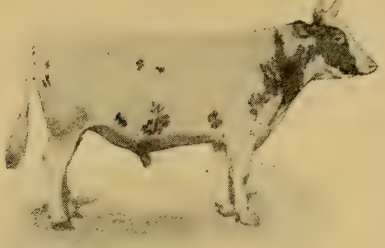

Fig. 9. Ayrshire bull, rough pasture than any other breed, though they respond quite as well to generous treatment with respect to abundant food supply. Ayrshire breeders have given much attention to the form and symmetrical shape of the udder, and have selected animals with large udders, straight and full at the back and level on the floor, extending well forward on the belly. The teats are cylindrical and placed far apart, but in very many cases are too short to be convenient.

In color, the Ayrshire is any combination of light red, red, red-brown, or dark brown with white. The dark color in the case of some bulls is almost black. Modern Ayrshire breeders distinctly prefer those animals in which the white color is largely predominating, and many modern Ayrshires might be described as white, with small patches of the darker color scattered over the body.

Ayrshire cattle were first brought to America by way of Canada some time previous to the middle of the ninteenth century, and shortly after they were introduced into the eastern United States. The numbers, however, remained small, although there are numerous localities in New York and New England 
where Ayrshires have been constantly kept since before the close of the Civil War. In those localities, where Ayrshires have been kept longest without importations from Scotland, they have taken on a rather different type. They have lost something of the smoothness of form, have increased somewhat in size, the udders have lost some of the symmetry, and dark colors usually predominate. Usually they have lost nothing in productivity, and some of the largest producers of the breed may be found in these localities. Animals of this sort are often spoken of as belonging to the American type in contradistinction to those first described, which are commonly known as of the Canadian or'Scotch type.

The Ayrshire ordinarily produces milk and butter in relatively large amounts for the amount of food consumed, but, considering the cow as an individual, the Ayrshire does not yield so much milk as the Holstein, and the milk is not so rich as that of the Jersey or Guernsey. Consequently they have to compete with all the other breeds, and they remain in point of numbers distinctly below the other three breeds in nearly all dairy localities.

Shorthorns. - While the Shorthorn ranks among the leading beef breeds, it should also be considered from the standpoint of the dairy. The original Shorthorn cow was a notable milk producer, and some of the earlier breeders gave attention to their development along this line, but the demand for beef overshadowed the dairy, and many tribes and families of Shorthorns have been bred so strongly for beef for 
so long a time that their dairy capacity has almost completely disappeared. On the other hand, a few breeders have maintained the milking qualities of their herds, and there are perhaps a dozen to twenty herds, scattered in various parts of the country, that are notable for dairy qualities, and are known as milking Shorthorns. In England, also, there has been a recent

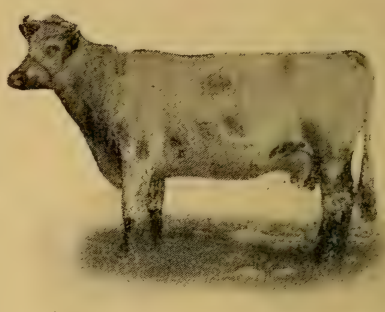

Fig. 10. Dairy Shorthorn cow renewal of interest in milking Shorthorns, and there are several notable herds in that country. Most of the Shorthorns that show good milking qualities trace their descent to families that originated in the herd of Thomas Bates, one of the earliest and most famous of Shorthorn breeders.

The Shorthorn originated in the valley of the river Tees, in the counties of Durham, Northumberland and Yorkshire, in northeastern England. They rank as the largest of the breeds of cattle. They have massive rectangular forms, short legs, short necks, and short, usually incurving horns. In color, they are usually spoken of as the red, white and roan. Their normal colors are red or white, or any combination of these colors, either in the intimate mixture known as roan, or spotted red and white. The red should be a clear red-brown, not shading to yellow on the one hand, nor to black on the other. At one time the clear red animals were much more sought 
after in the United States. At the present time there is no particular preference to colors or mixtures of colors.

The milk of Shorthorn cattle is of moderate richness in quality, and in quantity must rank below the Holsteins and Ayrshires.

Red Polled.-Red Polled cattle, as their name indicates, are a clear red hornless breed. They originated in the counties of Norfolk and Suffolk in eastern England, where they have been bred with considerable care for from fifty to seventy-five years. They are of medium to large size, ranking just below the Shorthorns, often have well-developed wedge-shaped forms, although many individuals approach too nearly to the beef type to be called first-class dairy animals. They are quiet in disposition, and their lack of horns makes them a favorite with some. In amount and quality of milk they rank with the better type of milking Shorthorns. There have been a few notable producers among them, but, as a rule, they cannot compete in amount of product with the leading dairy breeds, and are found in only small numbers in the United States.

Brown Swiss.-Brown Swiss cattle are native of the forest-cantons of Switzerland, notably the canton of Schwyz, where dairying has been extensively developed upon the mountain pastures. They are a large breed, with very heavy, coarse bones, thick hides and large extremities. They are quiet in disposition. In color, they are a uniform grayish brown, with a ring of lighter hairs about the muzzle, and shading to 
black on the legs and tail. Their bodies are rather heavy and somewhat beefy, though occasional animals show the distinctive dairy form. As a rule, they are not large producers, although occasional individuals are found that rank well up with individuals of the leading dairy breeds. There are comparatively few herds in this country. Their owners, however, are strong partizans of the breed, and claim for them the advantages of docility, hardiness and constitutional vigor.

Dutch Belted.-Dutch Belted cattle are a race resembling the Holsteins, and probably closely related to them. They are distinctly smaller in size, and the black and white colors are segregated in black extremities, and a broad white band about the middle. They are found in Holland, usually on large private estates, and are there known as Lakenvelders. They were introduced into the United States with the early Dutch settlers about New York, and have shown such power of transmitting their external characteristics that they maintained themselves practically pure, with no herd book organization, until the latter part of the nineteenth century. In general characteristics they resemble the Holsteins, but their smaller size and smaller production do not enable them to compete with the more important breeds. They are kept in small numbers by those who fancy their peculiar markings of form.

Devons.-Devon cattle, as the name indicates, originated in the county of Devon in sonthwestern England. They are also found to some extent in Corn- 
wall, Somersetshire and Dorsetshire. They have been developed from the native cattle of the country, along both beef and dairy lines. They are medium to small in size, have very neat, symmetrical, smooth, round forms, in which respect they somewhat resemble the Ayrshire. They are also sprightly and active in disposition, and unusually intelligent. Theír horns are long, ivory-white, slender and upright. They give a moderate quantity of fairly rich milk. Undoubtedly Devons, or cattle similar to them, were among the first imported into the United States from England by the early colonists. As pure-breds they have existed for more than one hundred years, but never in very large numbers. Because of their docility and capability for training, they have always been favorites in localities where oxen are used for labor. At the present time, as pure-bred animals, there are very few remaining.

From time to time other breeds have been represented in this country, but they never have been numerous enough to form any important part of dairy stock. Among such breeds may be mentioned the Normandy, the Simmenthal, the French Canadian, and the Kerry.

The Normandies are a medium-sized, parti-colored breed from Northern France. Simmenthal coming from the valley of the Simme, in Switzerland, resemble very closely the Brown Swiss in form and character, They are fawn and white in color. The French Canadian has been bred in the French provinces of Canada, since the time of the first settlers, from animals 
brought from France at that time. They are a hardy, active race and are good and profitable producers. They give promise of future development. The Kerry is the diminutive cow of the Irish peasant and is more curious than useful in this country. 


\section{CHAPTER IV}

\section{THE TESTING OF MILK}

MrLK is so variable in composition, and so easily adulterated, that it frequently becomes of great importance to be able to ascertain with a fair degree of accuracy the composition of any given sample.

\section{HISTORY OF MILK TESTS}

Gravimetric analysis.-The most accurate way to determine the composition of milk is by means of an exact chemical analysis. The constituents of milk which it is most frequently necessary to determine are the total solids and fat. The total solids are determined by drying an accurately weighed portion of the milk at the temperature of boiling water until it no longer loses weight. The residue is the total solids, and its weight, divided by the weight of the original amount taken, will give the percentage of total solids. The fats may then be determined by extracting the residue with anhydrous ether until nothing more is dissolved, and then evaporating the ether and weighing the resulting fat directly. Various forms of apparatus for making these determinations have been devised by vari- 
ous chemists, a considerable number of which give very accurate results. Chief among these methods for determining fat and total solids are the Babcock asbestos method and the Adams paper-coil method. In order that these determinations may be made with accuracy, balances of extreme delicacy, and apparatus more or less complicated and requiring considerable skill in its manipulation, are necessary, so" that for ordinary commercial purposes they are practically out of reach.

History of milk tests.-Although consumers of milk had felt for a long time the necessity of some means of protection against dishonest dealers, it was not until the development of the factory system of manufacturing cheese (1850) and butter (1870) that some means of easily determining the composition of milk, particularly as to fat content, became important to both producers and manufacturers. From that time on various methods have been devised, from the simple expedient of raising the cream in a small sample of milk in a graduated glass to apparatuses almost as complicated and difficult of manipulation as the gravimetric methods themselves.

Cream gauges.-The simplest and one of the earliest methods used to determine the quality of milk is to set a small portion of it under such conditions that the cream would be thoroughly thrown to the surface and easily measured. These were known as cream glasses, cream gauges, or creamometers, and to a certain extent served a useful pur- 
pose; but it was soon found that the percentage of cream depends not so much upon the amount of fat present in the milk as upon the size of the globules and the conditions under which they are brought to the surface, and that the percentage of cream does not necessarily bear a constant relation to the percentage of fat.

Specific gravity.-The determination of the specific gravity was next brought into use as a means of determining the quality of milk. Inasmuch as milk is slightly heavier than water, and as water is the most common adulterant of milk, any addition of water to it would serve to lessen its specific gravity, and would easily be detected by a determination of the specific gravity. To determine the specific gravity of milk, various forms of specific gravity hydrometers, known as lactometers, have been devised. They were formerly very much more depended upon as a test of the quality of milk than at present, and though now. we have learned that under certain conditions a simple specific gravity test may not only be inaccurate, but entirely misleading, still they are of considerable use for certain purposes and in connection with certain other instruments.

Lactometers. - In devising the lactometer, it was assumed that 1.029 was as low as the specific gravity of any unadulterated milk would ever fall; therefore a hydrometer was devised, the scale of which was graduated from 0 to 120 , the 0 marking the point of pure water, or a specific gravity of 1.000 , and 100 cor- 
responding to the assumed least specific gravity of milk, or 1.029 . If, then, in any given sample of milk the lactometer fell to 90 , it would indicate the presence of 10 per cent of water ; if it fell to 75 , of 25 per cent of water, etc. This form of lactometer is now known as the common, ordinary or Board of Health lactometer. A second form of lactometer in common use was devised by Quevenne, and bears his name. The scale of this hydrometer is ordinarily graduated from 15 to 40 , the 29 being coincident with 100 upon the ordinary lactometer and with

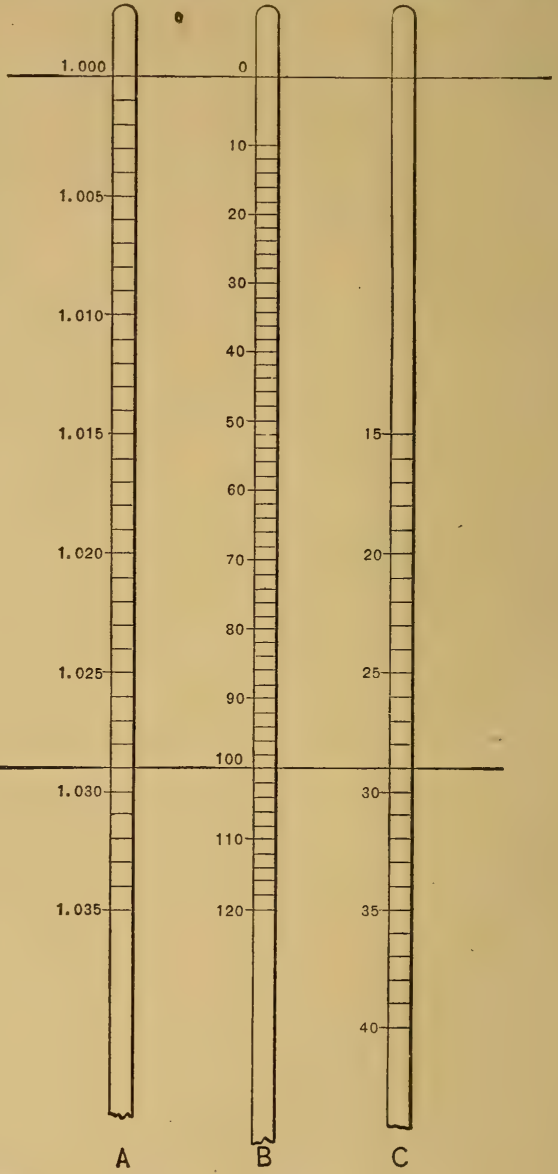

Fig. 11. Comparison of graduation on lactometer stems : $A$, hydrometer $\cdot B$, ordinary lactometer $C$, Quevenne lactometer. 
a specific gravity of 1.029 upon the ordinary hydrometer. The accompanying sketch (see opposite page) shows the relative values of the degrees upon the ordinary hydrometer, the ordinary lactometer and the Quevenne lactometer. With the Quevenne lactometer the specific gravity of the milk can be at once read, a degree upon this scale being equivalent to one degree of specific gravity. Since 100 degrees upon the ordinary lactometer indicate a specific gravity of 1.029 , the specific gravity of any ordinary lactometer realing may be obtained by inultiplying the reading by .29 , dividing by 1,000 and adding 1 . Twenty-nine Quevenne degrees are also equivalent to 100 ordinary degrees, so that Quevenne readings may be changed to the ordinary readings by dividing by .29 , and ordinary readings may be changed to Quevenne readings by multiplying by 29 .

The relative density of milk varies with its temperature, so that a hydrometer is only correct at one given temperature. Nost hydrometers are graduated for a temperature of $60^{\circ} \mathrm{F}$., and the better forms have an attached thermometer; so that if the milk to be tested varies from this in either direction a correction must be made. As the density increases with a reduction of temperature and decreases with a rise of temperature, the correction must be subtracted in going from a lower to a higher and added in going from a higher to a lower temperature. The amount of such correction for the Quevenne lactometer is .1 of a lactometer degree for each degree of temperature, and for the ordinary lactometer one lactometer degree for each 3 
degrees of temperature: In no event should the temperature of the milk to be tested be more than 10 degrees warmer or colder than the standard, and it is much better if the temperature does not vary from the standard more than 5 degrees in either direction.

The solids in milk are not all of the same specific gravity; some are heavier and some lighter than water. The fats are lighter, the other solids are all heavier. The specific gravity of the milk, then, depends not only upon the amount of solids present in the milk, but also upon their relative proportions. The specific gravity of milk may be affected by the addition of any substance to it or the abstraction of any of its constituents. Since some of the constituents of milk are lighter than water, their abstraction in whole or in part would be followed by an increase in density. It will be readily seen, then, that if a part of the fats are removed, the specific gravity of the skimmed or partly skimmed milk will be heavier than normal, and the addition of a certain amount of water or other substance lighter than the milk would only serve to bring the specific gravity back to the normal point. In this way, if it is done skilfully, water may be added to milk, and cream abstracted from it, without affecting the specific gravity as revealed by the lactometer, and a very inferior sample of milk might pass as perfectly normal if the lactometer alone were depended upon for its detection. Mainly for this reason the lactometer has been superseded by other and more accurate instruments. 
Churn tests. - The first butter factories or creameries were managed upon what is known as the cream-gathering system: that is, the cream was raised and skimmed upon the farm, and it alone taken to the factory. It was soon found that the cream varied considerably in the percentage of fat that it contained, and, moreover, that a considerable amount of milk could be mixed with the cream without being detected by ordinary means. In other words, the managers of factories learned that cream as it came to them was even more variable in its percentage of fat than whole milk. In all of the earlier factories the cream was paid for simply by measure, and it became necessary to devise some means of making an equitable division among the different patrons, and of protecting the factory from loss. To do this, what was known as test churns were devised. At the time of gathering the cream, a small sample (a pint or quart) of each patron's cream was taken in a separate vessel. These were taken to the factory and churned separately in small tin cans, and the butter made up from each. The butter-producing power of the single pint or quart was taken as a measure of the butter value of the whole amount of that patron's cream, and the proceeds were apportioned accordingly. This method was much more just than a simple measure of the cream, but it was very cumbersome. It required delicate manipulation in order to make all of the little pats of butter of the same water content, and the small 
amounts of butter so made were of inferior commercial quality, could not be mixed with the whole mass of butter, and entailed a considerable loss upon the creamery.

The oil-test churn was an outgrowth of this method, intended to remedy its defects, and was in a great measure successful. In operating the oil-test churn, the individual samples taken from each patron were very much smaller, and were taken in small glass tubes. These tubes were put in a frame and agitated until the fat was drawn together in a solid mass; the tubes were then immersed in water sufficiently warmed to melt the fat, and when so melted the fat would float upon the surface of the liquid in the tube. The tubes were allowed to become cool, were then a second time agitated to churn any particles of fat that had escaped the first churning, and the fat remelted; it then appeared in the form of a clear layer of liquid upon the top of the contents of the tube, and could be readily measured. The proportion of melted fat so obtained was taken as a measure of the butter value of the cream of which it was a sample. This test was generally used in cream-gathering factories, and was a very fair measure of the butter value of the cream. There was always a portion of the fat remaining unchurned, but in cream it was a small percentage. In milk, however, it was a much larger proportion, and the oil-test churn was never successfully used for äetermining fat in milk. 
Inventions for testing milk.-Several instruments of European invention have been described for the quick determination of the fat in milk. Some of them make volumetric determinations of the fat or cream ; others depend simply upon the opacity of the milk. One or two are in common use in Germany and Denmark, but, though most of them have been introduced in the United States, none have come into general use. The more importaut of these are the following:

Marchand's lactobutyrometer. - This is an instrument for quickly determining, volumetrically, the fat in milk. A measured sample of milk is introduced into a long glass tube graduated at the upper end. A certain amount of acetic acid is added and thoroughly mixed with the milk, after which ether is added to dissolve the fat, and with the aid of a small amount of heat the fat is collected into the upper graduated portion of the tube and read off volumetrically. The lactobutyrometer was introduced about 1877, and was used with more or less success for a time. In certain samples of milk it was found to be difficult to get a clear separation of the fat, and in certain other samples, notably the milk produced from various foods, it was found that the results could not be relied upon.

Heeren's pioscope.-This is a simple little instrument designed to test the quality of milk by means of its opacity. It consists of a hard rubber dise, in the center of which is a small depression, 
and the surrounding circle painted in segments of varying shades to represent cream, very rich milk, normal milk, poor milk, ete. A drop of the milk to be tested is placed in the central depression and covered with a glass plate, so that a layer of uniform thickness is always obtained. The opacity of this drop of milk upon the black background of rubber is then compared with a corresponding segment of the eircle. In so far as the fat measures the opacity of the milk, this is a fairly reliable test; and, used in connection with a specific gravity lactometer, a person with some experience can readily detect suspected samples of milk, although, of course, it is not possible to estimate very closely the amount of adulteration or the quality of the milk. The pioscope has been used very generally and successfully by milk inspectors and those having the control of city milk supply.

Feser's lactoscope is another instrument designed to determine the quality of milk by opacity. It consists of a glass eylinder, in the center of which is fixed a white rod graduated with black lines. A certain amount of milk is put into the cylinder, and by its opacity renders the black lines upon the central standard invisible. Water is then added to the milk in measured quantity until the black lines can be seen, the amount of water so added indicating the quality of the milk. This instrument is more delicate than the pioscope, but it can not be so quickly and readily used. The results it gives are of very much the same nature and 
value as those obtained by the use of the pioscope, sonsequently it has never been used to any great extent.

Soxhlet's method.-In testing milk by this method, the fat in a measured quantity of milk is dissolved in ether, the specific gravity of the ether solution determined, and from this the percentage of fat is calculated. The greater the specific gravity of the ether solution the greater the percentage of fat, and since the difference in the specific gravity of fat and ether is considerable, the addition of a small amount of fat will perceptibly affect the specific gravity, so that the determination is a very delicate one. The determination is made in a specially devised apparatus known as Soxhlet's Aërometer. It has been widely adopted in Germany, but not at all in the United States outside of chemical laboratories.

DeLaval lactocrite.-This is a machine devised by the inventor of the centrifugal separator to estimate the fat in milk volumetrically. The sample to be tested is put in a glass tube with an equal amount of concentrated acetic acid containing 5 per cent of concentrated sulphuric acid, and the mixture heated for a few minutes, after which it is whirled in a centrifugal machine until the fat is brought to the center. It is then read off volumetrically. The lactocrite gives a very close determination of the fat in milk, but it is necessary to have a separator frame in which to whirl the apparatus, which makes it somewhat expensive. 
Fjord's control apparatus.-This apparatus, in. vented by the late Professor Fjord, of Denmark, estimates the fat by measuring the solidified cream. Glass tubes similar to those used in the oil-test churn are secured in a frame and a measured quantity of milk put in each. The frame holding the bottles is then whirled in a centrifugal separator frame till the cream is completely separated and brought together in a compact mass. This requires about forty-five minutes. The solid mass of cream is then measured with a scale and the fat estimated from it by means of a table constructed by the inventor. This apparatus is in very common use in Denmark, but has never been introduced into this country.

Development of milk tests in the United States.-Up to the year 1888, there had been no apparatus devised which would determine the fat in milk accurately; easily, cheaply and quickly. None of the methods described could in any sense supply the place of the gravimetric analysis, even for commercial purposes. The oil-test churn came the nearest to it, but that was of no use for milk, and at this time the separator creamery was beginning to supplant the gatheredcream factory, and the demand was constantly stronger for a means of determining the fat in milk. This year also marked the establishment of the national grant to Agricultural Experiment Stations in each of the states, and one of the first problems attacked by the chemists of these stations was to devise a quick method for the determination of fat in 
milk. In the next two years no less than seven dis tinct methods were devised by chemists of Agricultural Experiment Stations for this purpose. All of them were much better than any that had hitherto been known, but one was so much in advance of any of the others that now it is practically the only method used for the quick determination of fat in milk. This is the method devised by Dr. S. M. Babcock, known as the Babcock test, and first published in July, 1890. The various tests, in the order of their publication, were as follows:

Short's method.-This method was invented by F. G. Short, at that time chemist of the Wisconsin Agricultural Experiment Station, ana was first published in Bulletin No. 16 of the Wisconsin Agricultural Experiment Station for July, 1888. In brief, the method consisted in converting the fat in the milk into a soap by means of an alkali, and then dissolving the soap by an acid, setting free the fat. The process was rendered complete by boiling for several hours. The determination was made with a measured quantity of milk in a glass test bottle with a narrow graduated neck, into which the fat was raised at the end of the process and read off volumetrically. Considerably difficulty was often experienced in getting a clear separation of the fat from the contents of the tube. The long period of boiling was also an important drawback to the method.

The method of Faityer and Willard.-This method was devised by Professors Failyer and Willard, of 
the Kansas Agricultural Experiment Station, and was published in the report of that station for 1888 . In it the solids of milk were destroyed by hydrochloric acid and the fat partially separated by means of heat. The fat was then dissolved in gasoline, and, after evaporation of the gasoline, was measured in a graduated portion of the tube in which the operation was performed. It gave a clearer reading of the fat than Short's method, but required more delicate manipulation, particularly in heating the acid and milk together and in evaporating the gasoline. The time required was considerably less than with Short's method.

Parsons' method.-This method was devised by Professor C. L. Parsons, of the New Hampshire Agricultural Experiment Station, and published in the report of that station for 1888 . This method made use of caustic soap and a solution of soap and alcohol to destroy the milk solids, after which the fat was dissolved in gasoline. A measured quantity of the gasoline solution of fat was then taken, the gasoline evaporated from it and the fat carefully dried. The free fat was then measured in a scale, and by means of a calculation, the percentage of fat determined. This method gave very good results in the hands of several different operators. It, perhaps, required a little more delicate manipulation than some of the others, and it was considerably more complicated than Short's method.

The Iowa Station test.-This test was invented by Professor George E. Patrick, the chemist of the Iowa 
Agricultural Experiment Station, and published in Bulletin No. 8, February, 1890, of the Iowa Experiment Station. In this test the solids of the milk were destroyed by a mixture of acetic, sulphuric and hydrochloric acids, and the fat brought to the surface by boiling. The test was made in a flask with a narrow graduated neck. A measured quantity of milk was put in the flask, a sufficient amount of the acids added, and the whole boiled for ten or fifteen minutes. The Iowa Station test was a great improvement upon any that preceded it in point of simplicity, accuracy and length of time required.

Cochran's method.-This method was invented by C. B. Cochran, of the Pennsylvania State Board of Health, and published in the Journal of Analytical Chemistry, Vol. III., page 381. In this method the solids of the milk other than the fat were destroyed by the use of a mixture of acetic and sulphuric acids, aided by boiling. When the milk solids were thus completely disintegrated, the fat was brought to the surface by the aid of ether, and then the whole mass further boiled until the ether was all evaporated. The clear melted fat was then measured by transferring it to a vessel with a graduated neck. The Cochran method was simple in details, but required rather delicate manipulation in transferring the melted fat from one vessel to another. It, however, gave very good results in a comparatively short time.

The Babcock test.-This test was invented by Dr. S. M. Babcock, chemist of the Wisconsin Agri- 
cultural Experiment Station, and published in Bulletin No. 24, July, 1890. In point of simplicity, accuracy, ease of manipulation and time required, this test is so much better than any that have preceded or followed it that it is now practically the only one in use. To destroy the solids other than the fat, Dr. Babcock makes use of a single reagent, commercial sulphuric acid, of a specific gravity of 1.82 , and to separate the fat from the remaining contents of the test bottle centrifugal force is used, hot water being added to bring the contents of the flask up to the graduated part. The test is made in a small flask with a narrow graduated neck.

The Beimling test.-This method of testing milk was devised by Messrs. Leffman and Beam, and is sometimes known under their name, though the apparatus was patented by $\mathrm{H}$. F. Beimling, and introduced under his name. The Beimling test was introduced in the year 1890, and was essentially like the Babcock test, the exception being that instead of a single reagent two were used, one ordinary commercial sulphuric acid, as in the Babcock test, and the other a mixture of amyl alcohol and commercial hydrochloric acid. Largely because of the greater inconvenience of using two reagents, the Beimling test has fallen into disuse.

Tests introduced since the Babcock test.-Two or three tests differing but slightly from the Babcock have been introduced since. One of these is known as Gerber's method, the invention of a German chemist. The form of the testing bottles differs 
somewhat from that used by Dr. Babcock; less whirling is required, and the same reagents are used as in the case of the Beimling. The DeLaval

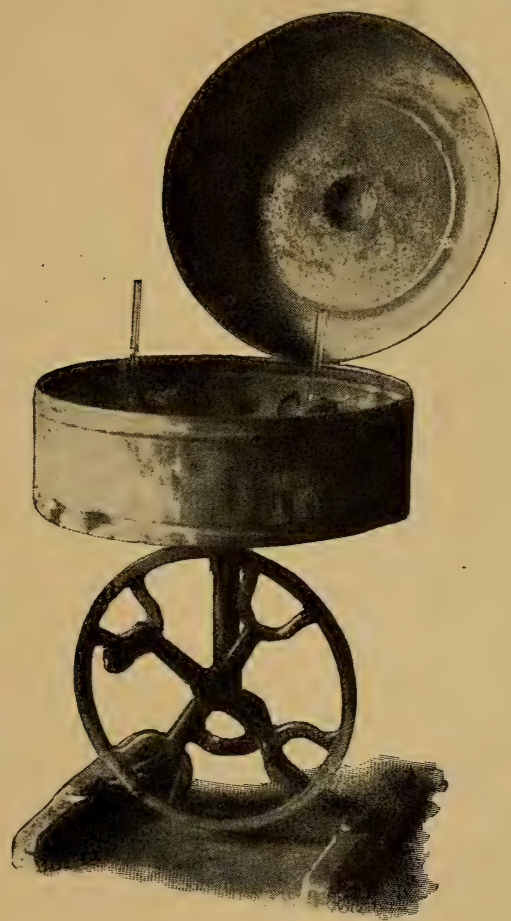

Fig. 12. Hand centrifugal for Babeock test.

Separator Company has also introduced an apparatus for testing milk which is known as the butyrometer. In this test a single reagent, sulphuric acid, is used to set free the fat. The form of the ap- 
paratus is different from the Babcock test, a much higher speed of whirling is used, a smaller sample of milk is taken, and the fat is read in a solid instead of a liquid form.

\section{DETAILS OF THE BABCOCK TEST}

The apparatus used in testing milk by the Babcock method consists of a centrifugal machine, three pieces of glassware and commercial sulphuric acid.

The centrifugal machine.-Many forms of centrifugal machines are in use, almost every manufacturer having his own particular style. It is essential that the centrifugal should be substantially made; that it should run smoothly and steadily, either loaded or empty, and that it should be capable of developing a speed of 900 revolutions per minute, with a wheel 20 inches in diameter. The centrifugals so made may be driven either by hand, electric, or steam power. In the hand-power machines, the motion is transmitted both by belts and by friction cogs; in the latter case it is essential that care be taken to prevent loss of motion through the friction cogs becoming worn.

In the centrifugals driven by steam turbines, or jets of steam delivered against the circumference of the revolving wheel, it is much better that the steam be applied at some little distance from the revolving bottles, otherwise too great a degree of heat may be developed in the machine. Woll has shown* that, in those steam turbines in which

\footnotetext{
*Hoard's Dairyman, Mareh 9, 1900 : vol. 31, p. 75.
} 
steam is introduced into the bottle chamber or where the cover fits so tightly that no cold air enters the chamber during the whirling; the bottles are often heated to such a degree that the reading is made too large because of the expansion of the fat at the high temperature. The fat should be read at a temperature of $110^{\circ} \mathrm{F}$., but up to $140^{\circ} \mathrm{F}$. the expansion is not sufficient to cause material error. When, however, the temperature rises to $200^{\circ} \mathrm{F}$. or thereabout, as frequently occurs under the conditions nanied above, the error due to the expansion of the fat may amount to .15 to .3 per cent. In all such cases the bottles should be allowed to cool to at least $140^{\circ} \mathrm{F}$. before reading. Those centrifugals are most

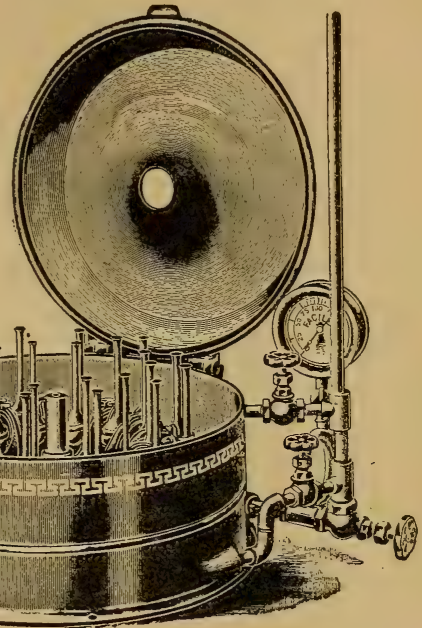

Fig. 13. Steam turbine centrifugal for Babcock test. (See opposite page.) satisfactory in which provision is made for the bottles to assume a perfectly horizontal position when in motion and a perfectly perpendicular one when at rest.

The glassware. - The glassware consists of a flask or test bottle in which the determination is made, a graduated pipette for measuring the milk, and a short graduated glass cylinder for measuring the 
acid. The most essential feature of the glassware is that it should be accurately graduated. This in general can be secured by always procuring the glassware from a reliable manufacturer or dealer,

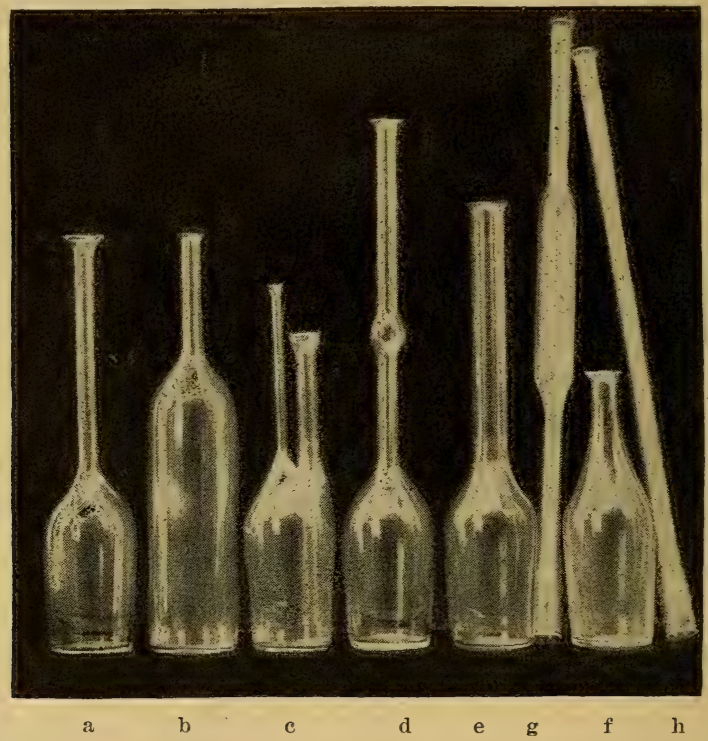

Fig. 14. Forms of Babcock test bottles ; $a$, ordinary bottle for whole milk; $b$, bottle for skim milk, using double charge of milk; c, Ohlsson, or "B. \& W." double-necked bottle for skim milk; $d, e$, bottles for testing cream ; $f$, bottle with detachable neck ; $g, h$, detachable necks for butter and cream.

though suspected glassware may be tested with comparatively little difficulty. The neck of the ordinary test bottle is graduated from 0 to 10 , each division being subdivided into five parts. The graduation from 0 to 10 will contain a volume of melted 
milk fat equivalent to 10 per cent of the weight of the milk taken. Each subdivision of the scale, therefore, represents .2 of 1 per cent. The capacity of the graduated portion of the neck is two cubic centimeters. The specific gravity of melted milk fat at a temperature of $120^{\circ} \mathrm{F}$. is assumed to be .9. The two cubic centimeters will, therefore, weigh 1.8 grams, and in order that the percentage of fat reacl off shall be percentage by weight and not by volume, 18 grams of milk must be taken. But milk has an average specific gravity of 1.032 , therefore 18 grams of milk will be contained in 17.44 cubic centimeters. Two cubic centimeters of melted milk fat is, therefore, 10 per cent by weight of 17.44 cubic centimeters of average milk. It has been found by trial that a pipette of the ordinary form graduated at 17.6 cubic centimeters will deliver slightly less than 17.5 eubic centimeters of milk. The graduation of the ordinary pipette should, therefore, be 17.6 cubic centimeters. A little less acid than milk is ordinarily required, and the acid measure is graduated at 17.5 cubic centimeters, though the amount of acid actually used may readily vary two or three cubic centimeters either way from this point.

The fat in the various products of milk may be as readily determined by means of this test as fat in the milk itself, and for these determinations various forms of special apparatus have been devised. (Fig. 14.) For testing cream, bottles with a capacity greater than 10 per cent are in use. Of 
these there are two forms. In one there is a bulb in the middle of the neck, and graduations above and below. Ordinarily the lower graduations have a eapacity of 5 per cent, the bulb a capacity of 10 per cent, and the upper graduations a capacity of 10 per cent. The use of this form of bottle requires that when the fat is read off the bulb should always be completely full, and the upper and lower surfaces of the fat rest on the upper and lower graduations respectively. Another form of cream test bottle has a neck much wider than that used for ordinary milk testing. Bottles of this form have a capacity up to 35 per cent, or even more. The graduations are usually not closer than .5 of 1 per cent. This form does away with the awkwardness of the bulb in the center, but it is not possible to read the column of fat to so small a fraction, usually to not less than .5 of 1 per cent. For testing cream, particularly cream that is rich in fat, a special pipette is necessary. The specific gravity of cream containing 25 per cent of fat or over is nearly that of water, and in testing cream of this quality a pipette of 18 instead of 17.6 cubic centimeters capacity is used. For testing skim-milk, where it is desirable to read the small fractions of 1 per cent, two forms of bottles have been devised. In one, two pipettes full of milk are used, and the graduations have one-half the ordinary value; in the other form, the bottle has two necks, one of ordinary width for the introduction of the milk and acid, and the other an extremely narrow one, in which 
the fat is measured. With this last it is possible to read easily to .01 of 1 per cent.

The fat in the solid milk products, as butter and cheese, may also be conveniently determined by the Babcock test. Since butter or cheese cannot be measured, it is necessary that the sample to be tested be weighed. Balances sensitive to .1 of a gram are sufficiently delicate. Either 18 grams of the substance may be weighed, in which case the percentage of fat is read directly from the bottle; or, what is more convenient, any amount from 4 to 8 grams may be taken. In the latter case, the observed reading of fat bears the same proportion to the percentage of fat in the substance taken that the weight of the sample taken bears to 18 ; and the percentage is found by multiplying the observed reading by 18 and dividing the result by the weight of the sample taken.

In testing butter and cheese, it is convenient to use the bottles with detachable necks. A little water should be added to the bottle before the acid is put in, to aid in the solution of cheese.

Calibration of glassware.-The correctness of the graduation of the glassware may be tested with more or less accuracy according to the means at hand. The bottles are all graduated on the assumption that the tubes are of uniform caliber. The 0 and 10 points are determined experimentally, and the intervening space equally divided into 50 divisions with a dividing engine. The spaces should, therefore, be of uniform size, and if the eye can detect 
any variation in the size of the spaces such bottles should be discarded. Bottles inaccurate in this respect are seldom met with now. When the test was first introduced they were of frequent occurrence. Bottles may be readily tested with a pipette of 2 c. c.* capacity. Fill the bottle carefully with water to the 0 point, wipe out the neck carefully, and drop in exactly 2 c. c. of water. It should just fill the neck to the top of the graduation. If delicate balances are at hand, the bottle may be weighed full of distilled or clean rain water to the . 0 point, and then again filled to the 10 point. The difference in weight should be exactly:2 grams. The calibration will be still more accurate if mercury instead of water is used; 2 c. c. of mercury may be measured out, or, what is still better, weighed. The specific gravity of mercury is 13.59 ; two c. c. will, therefore, weigh 27.18 grams. This weighed or measured quantity of mercury is introduced into a dry bottle, a close-fitting plug is then inserted into the neck of the bottle exactly to the top of the graduation, the bottle is then inverted; the mercury should exactly fill the graduated space. The same portion of mercury ean then be used to test another bottle, and with care to have the bottles dry, and to see that all of the mercury is transferred each time, a large number of bottles can be easily and quickly calibrated.

The pipettes are best tested by weighing a pipette full of either water or mercury; the former

* Cubic centimeter. See metric system, in Appendix. 
should weigh 17.6 grams, the latter 239 grams. Any bottle or pipette that varies more than 2 per cent from the standard should be discarded.

Sampling the milk.-The accuracy of the test depends wholly upon getting an accurate sample of the milk to be analyzed. A part of the fat so readily separates from the milk in the form of cream that milk cannot stand even for a short time without the upper layer becoming richer and the lower layers poorer in fat. Even in milk freshly drawn from the cow, that in the upper part of the pail will be considerably richer than that below. The first step, then, in sampling milk is that it should be evenly and thoroughly mixed. This is best brought about by pouring from one vessel to another: but if the milk has stood over night and a layer of tough cream formed, the particles of cream will not be thoroughly mixed by a single pouring from one vessel to another. In all such cases, the sampling must not be done until all visible portions of cream have disappeared in the mass of the milk. Various forms of sampling tubes or "milk thieves" have been devised for taking -samples of milk. They serve the purpose fairly well, but are not to be depended upon in comparison with a thorough agitation of the milk.

Where the previous night's milk is earried to the factory, the agitation enroute and the stirring incident to pouring from the carrying can into the weigh can are ordinarily sufficient to cause a pretty complete mixture of the milk: but in cold weather 
it will frequently be noticed that the cream is not thoroughly broken up. Under such conditions, extra precautions must be taken to secure perfect sampling.

Composite sampling.-In testing milk at factories, it is more convenient to take a sample every day, and make one test of the mixed samples at the end of a week, ten days or two weeks. In order to do this, it is necessary to provide a suitable receptacle for the milk of each patron. (Pint lightning-top fruit jars or milk bottles, or glass-stoppered sample bottles, are most convenient.) To these bottles is added each day a small portion of each patron's milk, together with some preservative for preventing the milk from souring. The preservatives in common use are bichromate of potash, corrosive sublimate, and milk preservaline. Caustic potash and soda may also be used. Neumann* claims to have had as good results with sodium nitrate as with bichromate of potash. Most of these substances are poisons, and render the milk unfit for use; the jar should, therefore, be plainly labeled. For this purpose colored corrosive sublimate is now in almost universal use. It gives a distinct color to the milk, and only a small quantity of it is necessary to prevent the milk from souring. Whatever preservative is employed should only be used in quantity sufficient to keep the milk from thickening. Of the bichromate of potash, an amount sufficient to color the milk a bright lemon yellow is all that is necessary. In taking

* Milch Zeitung, vol. xxii. p. 526. 
composite samples, an amount proportionate to the amount of milk delivered should be taken each day. This is conveniently done by the use of the Scovell Aliquot Milk Sampler, which, besides serving this purpose, gives the advantages of a milk thief in that it takes milk from all parts of the vessel. Where the milk varies only a few pounds from day to day, good results may be obtained by taking a uniform amount of milk for the sample each day, but where the variation in quantity is considerable, aliquot samplers are much to be preferred.

Making the test.-In preparing to make the test, the same care must be used that the sample shall be thoroughly mixed and perfectly uniform, that was taken in mixing the milk when the sample was drawn. In measuring the milk, the pipette should either be perfectly dry, or rinsed out with the milk to be tested immediately before measuring the assay. Where a large number of samples are to be tested, the latter is found to be the better practice. The greatest care should be taken that the milk is accurately measured. The lower end of the pipette should be placed about midway of the sample of milk and the pipette filled by gentle suction at the upper end. The milk should be drawn into the tube above the mark on the neck, and the end of the forefinger quickly placed over the end of the pipette, the pipette being steadied by the thumb and second and third fingers; holding it now on a level with the eye between the eye and the light, the pressure on the forefinger 
should be gradually relaxed and the milk allowed to flow out of the lower end drop by drop until the upper edge of the milk rests exactly upon the graduated mark on the side of the pipette. The milk is then transferred to the test bottle, and this should always be done by placing the end of the pipette against the side of the neck of the bottle, relaxing the pressure of the forefinger gently at first, allowing the milk to flow down the side of the neck. If this is not done there is danger that the neck will become clogged, and a part of the milk be blown out by the escaping air. When all of the milk has flowed from the pipette, the last few drops should be gently blown into the neck of the test bottle. The utmost eare must be taken that all of the milk is transferred from the pipette to the test bottle, and none allowed to escape.

The acid.-The next step is the addition of the acid. The acid should be put into the test bottle in such a way that it will rinse down any milk that has adhered to the sides of the neck, and pass between the milk and the glass in reaching the bottom of the bottle. As soon as the acid is added, the milk and acid should be shaken together with a gentle rotary motion until all of the curd is completely dissolved, care being taken that no particles of curd are thrown into the neck of the bottle. The amount of acid used should be about the same in volume as the milk, depending somewhat upon its strength. Ordinary commercial sulphuric acid with a specific gravity of 1.82 will require about 
17.5 cubic centimeters to completely dissolve 17.6 cubic centimeters of milk. If the acid is too weak the curd will not be completely dissolved, and will appear as a curdy, flocculent precipitate mixed with the lower part of the column of fat. If the acia is too strong, some of the solids of the milk will be charred, and will appear as dark-colored, flocculent particles, either mixed with the fat or immediately under them, when the test is completed. Slight differences in the strength of the acid may be overcome by adding a little more or a little less, according as it is too weak or too strong, but satisfactory results cannot be depended upon unless the acid is of the right strength; viz., $1.82 \mathrm{sp}$. gr. Convenient hydrometers may be secured for a trifling amount, so that any one may be certain of the strength of his acid. The sulphuric acid should be kept tightly corked in a glass-stoppered bottle, because when exposed to the air, it takes up water rapidly, and soon becomes too weak. While it is not necessary that the sulphuric acid should be chemically, pure, some of the cheaper grades of commercial acid often contain impurities that seriously affect the results, causing black specks to appear in the neck of the bottle. A reasonably pure commercial acid should always be used, and can be secured at a trifling cost above the impurer forms.

Whirling.-When the acid has been added and thoroughly mixed with all of the samples, they are put into the centrifugal machine and whirled steadily for five minutes. At the end of this time the ma- 
chine is stopped, and the bottles are filled with warm water to the bottom of the neck. They are then whirled a second time for two minutes, when water is again added up to about the 8 per cent mark on the neck of the bottle, after which they are given a whirl for one minute. The bottles are then taken out and - - - read as rapidly as possible.

Reading.-The reading should be taken at a temperature between $110^{\circ}$ and $140^{\circ}$ F., at which temperatures the fat will be completely fluid. The test should be made in a room at a temperature not less than $70 \mathrm{~F}$., or if the room is much colder some means should be taken to prevent the bottles from becoming cool until all are read. Much skill and facility can be attained by practice in reading the bottles rapidly and accurately. In reading the bottles, the reading should be made as shown in the diagram, the lower reading Fig. 15. Dia- from the extreme lower curved surface gram of neek
of Babcock $(a$, Fig. 15), and the upper reading from test bottle:
the reading the extreme top of the column of fat, should be $(b$, extrene to made be- $(b$, not $c$, Fig. 15), the difference between tween the
points $a \cdot b$, the lower and upper reading giving the points
not $a-c$. percentage of fat. The reason for reading in this way is that a small amount of residual fat is left mixed with the other fluids in the bottle. This is composed of the smaller globules of fat, and the amount is practically uniform, and has been 
found to represent about the amount occupied by the curved surfaces in the neck of the bottle, due to the capillary attraction between the fat and the glass.

Cleaning the glassware.-Good results cannot be secured unless the glassware is kept clean and bright. This can easily be done with very little trouble. As soon as the bottles are read, and while they are still hot, the contents should be emptied out. The hot acid and water will carry out with it the larger part of the fat in the neck of the bottle. The emptied bottle should then be rinsed once in warm water and once in hot water containing some alkali, either washing soda or any of the various washing powders, and then rinsed with either warm or cold water until they are perfectly clean. With these precautions no difficulty will be experienced in keeping the bottles clean and bright.

By the use of the lactometer in connection with the percentage of fat, a close approximation to the percentage of total solids, or solids not fat, may be made. Numerous formulæ for this purpose have been devised. Their application is explained and illustrated in Part $A$ of the Appendix. 


\section{CHAPTER V}

\section{THE FERMENTS AND FERMENTATIONS OF MILR, AND THEIR CONTROL}

Milk, when it is first drawn, is a limpid fluid with a slight odor, mildly sweetish taste, and faint alkaline reaction. In fact, milk often shows the amphoteric reaction; that is, it will give the acid reaction with blue litmus paper and the alkaline reaction with red. Almost immediately after - it is drawn, milk begins to undergo a change, and within a short time will show a distinct acid reaction. The degree of acidity increases with the age of the milk. Soon changes begin in the other milk constituents, and in a comparatively short time, the decomposition is so great that the milk can no longer be used for food. The sugar is the first constituent of the milk to undergo change, afterwards the albuminoids are attacked, and lastly the fats. These changes are not due to any instability of the organic compounds in the milk, but to the effect of various vegetable germs that gain access to the milk after it is secreted, and, living and growing in the milk, bring about the changes mentioned. These changes are called fermentations, and the agents that bring them about ferments. Milk containing no germs of fermentation, or milk from (108) 
which they have all been removed, is said to be sterile. The germs found in milk belong to the lowest orders of the vegetable kingdom. Most of them are included in the bacteria, although many yeasts and moulds are frequently found in milk.

The bacteria.-The bacteria are extremely minute bodies consisting of a single cell filled with protoplasm. They are of three general forms,- spherical (coccus); rod-like or cylindrical (bacillus), and curved or wavy (spirillum). They reproduce by fission; that is, the cell elongates slightly in the direction of its longer axis, and a partition is formed across the cell transversely, and two individuals exist where there was but one before. These may break away and form separate bacteria, but often they are kept together in various ways. Many forms are endowed with motion, and all require a liquid or semi-liquid medium for growth and development, though many may exist for long periods of time in a dry condition. Like other plants, in order to grow and develop, the bacteria must have suitable food. They require for their sustenance carbon, hydrogen, oxygen and nitrogen, together with small amounts of mineral matters. Organic compounds are more available for food supply than simple inorganic salts. Substances like sugar and the various albuminous compounds are admirably suited for their food. In ordinary milk nearly all kinds of bacteria find an adequate and easily available food supply in a medium favorable to their growth, so that not only the forms of bacteria ordinarily found in milk, 
but almost any others, will readily live and grow should they gain access to milk. Nearly all forms of bacteria, are sensitive to conditions of temperature. The range of temperature in which they thrive the best and grow most rapidly is rather narrow, though there is a considerable range above or below, in which they will still grow and develop. The temperature at which any given germ will grow most rapidly is called the optimum temperature, and the optimum temperature varies widely with the various classes of ' organisms, though by far the larger number of bacteria find their optimum point between $75^{\circ}$ to $100^{\circ} \mathrm{F}$, and a higher temperature not only stops their growth, but if sufficiently high kills them outright. A temperature of $135^{\circ} \mathrm{F}$. kills a large number of germs. Very few are able to live above a temperature of $180^{\circ}$, and none can withstand the temperature of boiling water, $212^{\circ}$, for more than a few minutes. If heat is accompanied by moisture it is much more effective, so that heat applied in the form of live steam is the best means of destroying the life of these germs. Under the influence of cold the germs become inactive, and some kinds are killed by a sufficient degree of cold, but very many kinds are able to withstand any degree of cold possible to be produced for long periods of time.

Under certain conditions bacteria are able to assume an inactive condition, or spore form. In doing this, the protoplasm shrinks into a hard, glistening mass, and contracts toward one end of the cell, 
or the cell wall thickens and encloses the protoplasm. In the spore condition the bacteria are in-. active, but are able to endure much greater extremes of heat, cold or moisture than when active. When the conditions of growth become again favorable, the spore again becomes active, or is said to germinate, and the vital processes are resumed.

Bacteria are widely distributed through nature. In fact, there are very few places where they may not be found. They are so light and small that they float readily in the atmosphere, particularly when accompanied by particles of dust. They are found in all rivers and streams, upon the surface of the earth, and upon all organic matter. In fact, they are universally distributed. By far the larger number are not only perfectly harmless but positively beneficial. They serve to transform dead organic matter into its original condition, and so act as scavengers. Others, like the milk ferments, bring about specific changes in some definite substances, while still others, a large class, are the specific causes of various diseases in men and animals.

Presence of bacteria in milk.-In ordinary milk, bacteria are always present in large numbers. These gain access to the milk from the atmosphere, from the bodies of the animal and the milker, through contact with the vessels into which the milk is drawn, and to some extent through the udder of the animal. The milk when secreted is sterile. So far as is known, no bacteria can pass through the digestive organs and blood vessels of the animal 
and appear in the milk. If the udder is the seat of disease due to the growth of bacteria, such bacteria may find their way into the milk ducts and infect the milk. In one other way the animal may be said to be a source of infection with bacteria. The end of the teat of the animal is always more or less moist. Bacteria coming in contact with such surface, moistened with milk, find there not only food in proper form for their growth, but a temperature sufficient to make them active. They begin to multiply, and, working their way through the orifice of the teat, find milk in larger supply, and a temperature still more favorable for their growth. They increase and multiply, under such conditions, with remarkable rapidity, and so work their way upward through the milk eistern and into the larger milk ducts, so that the milk first drawn from the animal always contains a greater or less number of bacteria. For this reason it is not an easy matter to secure perfectly sterile milk direct from the cow, though with great care in disinfecting the udder and removing the larger part of the milk from it, perfectly sterile milk has been obtained.

Kinds of bacteria in milk.-Almost any of the known forms of bacteria may live and grow and occasionally be found in milk. Normally, however, comparatively few forms of bacteria are present. The greater part of these are forms which cause various changes in the constituents of milk, and are known as ferments, and the changes which they induce as fermentations. Beside these fermentations, there may 
be found in the milk the bacteria of any germ disease with which the animal may be afflicted, or which may be carried into the milk through the atmosphere, the water used in cleansing utensils, or the persons of individuals suffering from the disease.

The fermentations of milk.-The normal fermentations to which milk is subject may be conveniently divided into three classes. First, those which feed upon and cause changes in the milk-sugar, known as lactic fermentations. Second, those that feed upon and cause changes in the albuminoids of the milk; these in turn are of two classes, peptogenic and putrefactive. Third, those which attack the fats, and are known as butyric fermentations. Besides these, which may be called normal fermentations, in that they will easily occur in any sample of milk if left to itself, there are a large number of other fermentations which may be called abnormal, from the fact that they occur only in isolated localities, or from time to time. These abnormal fermentations include one which causes the casein of milk to coagulate without the development of lactic acid, known as sweet curdling; another which causes a peculiarly ropy or slimy condition of certain constituents of milk; still another that results in the formation of an intensely bitter product in the milk; an alcoholic fermentation, and several fermentations which result in the production of various colors, collectively known as chromogenic fermentations. An illustration of this class is seen in the well-known "bloody bread," which is caused by the growth of Bacillus prodigiosus. 
This germ is occasionally found in milk, and imparts to it a red color which is easily confounded with the red color due to the presence of blood from a wounded udder. A single germ rarely occasions more than a single fermentation. Often two or more are combined in the fermentation, and in many cases there are a large number of different germs that bring about the same fermentation. This is notably true of the lactic and putrefactive fermentations. The effect of the various fermentations is such as to destroy the value of the milk as such, if they are allowed to proceed to any great length; but the manufacture of butter is greatly aided by many of these fermentations, and the presence of certain germs is absolutely indispensable to the manufacture of cheese.

Relation of mill bacteria to the human system.-By far the greater number of germs ordinarily found in milk are absolutely harmless, and may be taken into the human system in large numbers with perfect impunity, the germs of specific disease excepted, and with these latter it is the products formed from their growth rather than the germs themselves from which danger comes. There are probably no germs normally found in milk that may be classed as harmful. This is also true of a considerable number of fermentation products resulting from the growth of the germs in the milk. Many of these products give to the milk or its product an unpleasant taste or physical appearance, but are otherwise perfectly harmless. There are, however, certain germs which produce a fermentation which results in the forma- 
tion of poisonous products. These products are the eauses of the serious or even fatal results that follow the consumption of milk, cheese, ice-cream, or other products containing them. They are collectively known as ptomaines. To one in particular, that has frequently been found in cheese, the name tyrotoxicon (cheese-poison) has been given. They have been studied by Vaughn* and others, but their origin is still obscure. The germs producing these poisonous products are of comparatively infrequent occurrence.

In general, the various classes of fermentations do not readily take place at the same time. The active growth and development of one germ acts more or less as a retarding force upon the growth and development of other germs.

Lactic fermentations. - Under this group we include all of those germs which, living and growing in milk, feed upon the sugar, causing it to change to lactic acid. It was formerly supposed that the formation of lactic acid in the milk was entirely due to the action of a single germ, described by Hueppe, and called Bacillus acidi-lactici, or the lactic acid germ. It is now known that there are at least twenty different germs that may produce lactic acid, and in all probability there are many more. The lactic acid germs are the most common and most numerous germs found in milk, and ordinarily the lactic fermentations are the first to take place. They begin their opera-

\footnotetext{
* Vaughn-Novy. Ptomaines and Leucomaines, Philadelphia, 1896.
} 
tions almost immediately after the milk is drawn, and continue until the maximum amount of lactic acid has been produced. In the lactic acid fermentations one molecule of milk-sugar $\left(\mathrm{C}_{12} \mathrm{H}_{22} \mathrm{O}_{11} \div \mathrm{H}_{2} \mathrm{O}\right)$ breaks up into four molecules of lactic acid $\left(\mathrm{C}_{3} \mathrm{H}_{6} \mathrm{O}_{3}\right)$ without the formation of any secondary or by-product. The presence of the lactic acid serves to coagulate the casein, so that curdling of the milk is always an accompaniment of the lactic fermentation after it has reached a certain stage. The presence of lactic acid is unfavorable to the growth of the ordinary germs of lactic fermentation, and when a certain amount of lactic acid has been formed (about 8 of 1 per cent of the whole milk), the further development of lactic acid ceases. In milk of ordinary quality, this occurs when about one-fourth of the milk-sugar has been changed to lactic acid. If the acid be neutralized with an alkali, the fermentation will then proceed until another portion of milk-sugar has been changed to lactic acid, showing that the lactic acid simply prevents the growth of the germs, and does not kill them. Lactic acid germs are most active at temperatures between $80^{\circ}$ and $100^{\circ} \mathrm{F}$.; at temperatures below $80^{\circ}$ they gradually lose their activity, and below $50^{\circ}$ little or no lactic acid will be formed. At these low temperatures they are simply inactive, not dead. At a temperature of $105^{\circ} \mathrm{F}$., the lactic germs become inactive, and a large proportion of them are killed at a temperature from $135^{\circ}$ to $140^{\circ} \mathrm{F}$. In milk, lactic acid fermentation means simply souring, and it renders the milk unfit for use, 
almost wholly because the taste is unpleasant to the ordinary palate. $\Lambda$ large amount of lactic acid is, perhaps, injurious to young and delicate or weak digestive organs, but ordinarily is harmless. 'Lactic acid fermentations are extremely important in the processes of both butter and cheese manufacture, and their relations to these processes will be discussed in detail in the proper place.

Fermentations affecting the albuminoids.-These include ordinary putrefactive fermentations, peptogenic fermentations, and fermentations resulting in the formation of poisonous products. These fermentations, as a rule, do not thrive in the presence of a strong lactic fermentation, so that ordinarily they do not manifest themselves in milk unless the conditions are peculiarly favorable for their development and unfavorable for the development of lactic acid. Many of the putrefactive fermentations will go on at a lower temperature than the lactic fermentations do; hence it is often found, when milk is kept at a low temperature in order to keep it from souring, that after a certain time it becomes bitter or foul-smelling. This condition is caused by some one of the characteristic putrefactive fermentations. The putrefactive germs also readily take on the spore form, and in this condition are not so readily killed by heat. The putrefactive fermentations usually result in the formation of bitter or other unpleasant flavors and disagreeable odors, and they are frequently accompanied by a considerable evolution of gas. Peptogenic fermentations are those which exert a pep- 
tonizing or digestive action upon the albuminoids. By their action the casein is first coagulated, and finally liquefied or changed into a peptone.

Butyric fermentations. - The butyric ferments attack the fats, and result in the formation of butyric acid. They produce the peculiar condition found in cream and butter known as rancidity, and do not usually manifest themselves very strongly in the milk.

\section{Control of Fermentations}

Since fermentations always occur in milk that is kept for any considerable time, and since they exert so powerful an influence, not only upon the milk but upon the products manufactured from it, the question of their control is one of prime importance. The three chief means of such control are: First, prevention of infection; second, prevention of the growth of germs already present; third, destruction of germs already present.

Prevention of infection. - The greatest source of infection comes from the body of the animal and from the air of the stable. The germs are always present, adhering to the hair of the animal, and particularly to any particles of dust or dirt. In order, then, that the animal shall not be a source of infection, it is necessary that she be carefully curried and the udder, teats, flank, thighs and lower parts of the belly wiped off with a damp cloth immediately 
before milking. It is of course necessary, also, that the hands and clothes of the milker should be as carefully attended to in this respect as is the body of the cow. The bacteria find in the excrements abundant food for growth and development, and are always found in large numbers where such excrements are allowed to collect; and when dirt of this sort accumulates and becomes dried, the dust floating in the air always carries with it large numbers of germs. The germs are also found in large numbers accompanying the dust arising from hay and other dried forage. This being the case, it is essential not only that the stable should be kept scrupulously clean, but that the air should be free from dust, particularly at times when milking is going on. Thoroughly sweeping and then sprinkling the stable floors an hour or two before the milking will materially lessen the germ content of the milk.

Next to the stable, the dairy utensils are an im. portant source of infection with bacteria. They become attached to the seams and corners of the vessels, and are not dislodged even with the most careful cleaning, and when the fresh, warm milk is drawn into such vessels the germs immediately begin to grow and develop. The most scrupulous care must, of course, be taken in cleaning any vessels in which milk is contained, but no vessel can be considered safe so far as conveying germs is concerned unless it has been exposed to the action of live steam for at least three minutes, and then kept in a secure place until needed for use. With these precautions 
in regard to the animal, milker and utensils, milk may be secured with a minimum number of bacteria. The difference in the number of bacteria in milk so drawn, and in milk carelessly drawn, may easily amount to a difference of eighteen to twenty-four hours in keeping quality under like conditions.

Holding at low temperatures. - If milk be immediately cooled to a temperature of $40^{\circ} \mathrm{F}$., or thereabouts, very little fermentation will go on, though it will be frequently found that after three or four days the milk or cream may have a more or less disagreeable flavor, due to the presence of some germs that develop slowly even at low temperatures. If low temperatures are to be depended upon as a means of keeping fermentations in check, it is, of course, of prime importance that every precaution should have been taken to prevent the access of germs to the milk in the first place. The fewer germs the milk contains to begin with, the more effective will low temperatures be as a means of preservation. With care in both these respects, milk or cream may be kept in a fresh and merchantable condition for a week or ten days.

Destruction of germs in the mill.-A large number of ehemical agents is more or less destructive to germ life. Many of them are so violent in their action as to destroy the milk as well as the germs, but there are many which are destructive to germ life, with no effect upon the composition, odor or flavor of the milk; but all of these without exception are more or less injurious to the human sys- 
tem, particularly if they are used continuously, even though only in small quantities. Of the compounds which may be used for this purpose, formalin, salyeilic and boracic acids and their derivatives are undoubtedly the least injurious, but their use is not to be recommended under any circumstances. Some attempts have been made to utilize the electric current as a means of destroying germ life in milk; but they have so far proved ineffectual, and instances are reported* where electrolysis of the milk constituents occurred where a continuous current was employed. Heat, then, is the only available agent that can be used for the destruction of germs already present in the milk. This destruction of germs in milk or any other fluid by means of heat is called sterilization. In order to absolutely sterilize any substance, it is necessary that it should be subjected to a heat of $212^{\circ}$ to $240^{\circ} \mathrm{F}$. for one hour on each of three successive days. This will kill not only the germs that are in active growing condition, but any spores that may be present. A lower temperature, $175^{\circ}$ to $212^{\circ} \mathrm{F}$., will kill actively growing germs, but even at this temperature chemical changes are set up in the milk which give rise to flavors known as boiled or cooked flavors, that are disagreeable to a large number of people. In order to overcome the bad effects of heating at such high temperatures another process, known as pasteurization, is used.

Pasteurization.-The name is taken from Pasteur,

*L'Industrie Laitière, April 1896. 
who discovered and used the process in controlling the fermentations of wine and beer. It differs from sterilization only in the degree of heat used, and, in fact, may be properly called an incomplete or partial sterilization. The destructive effect of heat upon germ life depends both upon the degree of heat and the length of time to which the germs are exposed. A large number of germs are killed at temperatures from $133^{\circ}$ to $140^{\circ} \mathrm{F}$., while others are killed at temperatures varying from $150^{\circ}$ to $165^{\circ} \mathrm{F}$. These latter temperatures include the germs of all of the ordinary ferments and most of the germs of specific diseases, including that of the tubercle bacillus. Since the tubercle bacillus is the disease germ most likely to be present, milk is ordinarily considered to be safe from disease germs when it has been pasteurized at a temperature sufficiently high to destroy it. This is a temperature of $149^{\circ} \mathrm{F}$. for thirty minutes, a temperature of $155^{\circ} \mathrm{F}$. for fifteen minutes, or a temperature of $167^{\circ} \mathrm{F}$. for ten minutes, and these temperatures have come to be looked upon as standard pasteurizing temperatures. Milk may be heated to $165^{\circ} \mathrm{F}$., if quickly cooled afterwards, without developing a boiled taste; so that it is possible that milk be rendered safe from the germs of disease and free from the ordinary germs of fermentation without developing in it a boiled taste. But in order to pasteurize milk safely, it is necessary that means should be provided for cooling rapidly from the pasteurizing temperatures to $50^{\circ} \mathrm{F}$. or below.

Milk carefully pasteurized, as above described, will 
remain sweet thirty-six to forty-eight hours longer at ordinary temperatures than milk not pasteurized, from which germs have been excluded with ordinary care.

The problem of successful pasteurization, then, depends upon the means of raising the milk in a short time to the required temperature, holding it there uniformly for ten or twenty minutes, and then cooling it rapidly to $50^{\circ}$ or below. Several forms of apparatus have been devised for this purpose. Some of them are fairly perfect, but most of them are lacking in some important point. With the present activity in regard to this subject we shall undoubtedly soon have much more perfect apparatuses for this purpose than are at present available. The perfect pasteurizing machine should cover the following points: Quick, perfect and uniform heating of the milk; perfect control of the temperature; quick and uniform cooling; compact form; ease of cleansing; absence of pumping arrangements; security against re-infection during the process.

Selection of milk for pasteurization.-For the best results in pasteurizing, it is also essential that the milk be as fresh and free from fermentations as possible. Russel and Farrington have found* that milk that has developed as much as .2 of 1 per cent of lactic acid is too sour for satisfactory results. Inasmuch as this amount of acid cannot readily be detected by the senses of smell or taste,

*Wisconsin Agr. Exp. Station, Bulls. 44 and 5\%. 
Farrington's alkaline tablets offer a very convenient means of selecting milks that are suitable or unsuitable for pastuerizing purposes. For this purpose it is convenient to make the tablet solution (see Chap. X.) by dissolving one tablet in each ounce of water, or one tablet in $30 \mathrm{c.}$ c. of water. Then with a cup or other convenient vessel and a small measure of any suitable size, the comparative acidity of different milks can be readily and quickly determined as follows: Put a measure full of milk into the cup and add two measures of the tablet solution. If the color disappears, more than .2 of 1 per cent of lactic acid is present, and the milk is too sour for pasteurizing purposes. If the milk remains pink, less than .2 of 1 per cent of lactic acid is present, and the milk may be safely used. If it is desirable to measure the amount of acid, each measure of solution may be roughly taken to represent .1 of 1 per cent of acid. Thus, if the pink color remains when one measure of the solution has been added, the milk contains .1 per cent of acid. If it require four measures of the solution before the pink color is permanent, the milk contains .4 per cent of acid. A convenient measure for this purpose is made by soldering a piece of stiff wire to the side of a No. 10 cartridge shell, after the manner of a milkmeasuring dipper. 


\section{CHAPTER VI}

\section{DETERMINATION OF BACTERIA IN MILK}

Bacteria as a measure of dirt in mill.-Dince a chief source of contamination of milk with bacteria is in dirty surroundings, the relative number of bacteria in milk has come to be largely accepted as an index of its quality with regard to cleanliness. As a matter of fact, in certain of the higher grades of the market milk, notably in "certified milk," the standard of quality is fixed by the number of bacteria per cubic centimeter found in the milk, and if the number so found exceeds a certain amount the milk cannot be graded as "certified." For these reasons it is necessary to make frequent determinations of the bacterial content of milk. This is best and most accurately done by a trained bacteriologist in a well-appointed and equipped laboratory. However, the simple determination of the number of bacteria in a given amount of milk is not a difficult operation, nor does it require a very elaborate or expensive equipment. There are reasons why a dairyman, particularly one producing certified milk, should have the means at hand for making his own determination of the bacterial content of his milk, the chief reason being that it enables him to control the conditions and work from day to 
day, so as to check carelessness in any place, and materially reduce the chances for contamination.

The outline of methods and apparatus given herewith will enable anyone with a little skill and ingenuity to make bacterial determinations sufficiently accurate for purposes of control in the production of high-grade milk.

The laboratory.-In fitting out a small laboratory for the determination of bacteria in milk, much can be left to the ingenuity of the individual. It is necessary to have the work done in a place as free from dust and other contamination as possible. So, if the room has to be used also for other purposes, it is well to shut off or enclose with glass a small portion to be used for this work alone, and in which the sterile glassware, media and water may be kept, so that everything is ready and convenient for use at any time.

Apparatus.-In choosing a sterilizer for glassware, any kind of an oven fitted with a thermometer, and in which a temperature of $150^{\circ} \mathrm{C}$. can be maintained, will do. For sterilizing media and water, an autoclave, that is, a steam sterilizer which is able to maintain a pressure of at least ten pounds per square inch, is most convenient; there are also on the market "high altitude" or Denver cookers that are satisfactory. If neither of these is available, steamers or simply boiling water may be used. It must be remembered, however, that, unless pressure is used, a greater period of heat is necessary, and that with substances such as gelatin and milk, which will not stand such treatment, the intermittent method of sterilization must be used. 
The lists of glassware necessary for plating one sample of milk and for preparing one liter of medium, together with the necessary materials and apparatus, are given at the end of this chapter, the actual amounts being left to the discretion of the individual. They may be readily secured from any firm dealing in such materials.

Some sort of an incubator is necessary. Very satisfactory incubators are specially built for bacteriological purposes, but, if it is necessary to economize, it is possible to convert a poultry incubator for the purpose, or a home-made incubator may be cheaply and simply built by anyone with a little ingenuity. An asbestos-lined box fitted with an electric light and thermostat is very satisfactory. The one factor necessary is a constant temperature of $37^{\circ} \mathrm{C}$. or $98.6^{\circ} \mathrm{F}$.

Media.-It is possible to obtain agar or gelatine already prepared from various bacteriological laboratories. If, however, it is found necessary to make the medium, the following directions may be used: As a basis for this medium use nutrient bouillon, or broth. This may be made either from the ground beef or beef extract. Directions for making the bouillon from ground beef are found in Moore's Laboratory Directions for Beginners in Bacteriology. It is much easier to use beef extract and, for general purposes, entirely satisfactory.

Measure out the following:

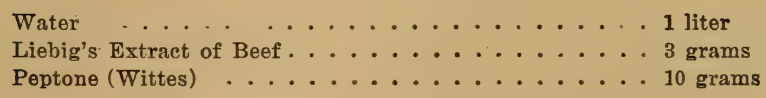


Place in the agate cooking-pan and weigh. Dissolve carefully at a temperature not over $150^{\circ} \mathrm{F} .\left(60^{\circ} \mathrm{C}\right.$. $)$

It has been found that organisms develop best at a reaction slightly but distinctly acid. That is, the media should be +1 per cent to phenol phthalein. In order to standardize the media to this degree of acidity, take 5 c. c. of bouillon, and place in a porcelain evaporating dish or white eup with about 45 c.c. of warm water. Add a few drops of phenol phthalein (8 per cent dry in 50 per cent alcohol). This is a solution which is colorless when acid, but tinted pink when alkaline. Stir and add to the solution in the cup enough of the twentieth normal sodium hydroxid solution from a burette to give it a clear, bright pink color. This is the amount needed to nentralize 5 c. c. of the bouillon. In order to bring the entire amount to +1 per cent, subtract 1 from the amount of twentieth normal sodium hydroxide used; multiply this by the number of cubic centimeters of bouillon, and divide by 100 . This result represents the amount of normal sodium hydroxide to be added. After adding the normal hydroxide, test the reaction again. It should take $1 \mathrm{c}$. c. of twentieth normal sodium hydroxide to bring a pink color with 5 c. c. of bouillon. This process is called titration.

After obtaining the desired acidity, boil briskly for twenty minutes, restore weight, with distilled water, cool and filter through filter paper into sterile flasks. The bouillon is now ready to be made into either gelatine or agar, or it can be sterilized and set aside until needed. (See directions for sterilizing media.) 
Lactose agar.-To 1 liter of bouillon add 1.5 per cent of agar ( 15 grams). Allow the agar to soak for one-half hour. Record weight, then add 10 grams of lactose. Steam for 20 minutes, cool to $60^{\circ} \mathrm{C}$.; titrate to +1 per cent acidity, add the white of an egg shaken up in 30 c. c. of water and steam for 20 to 30 minutes; then boil until clear, taking care not to burn it. Restore weight by adding water, then filter through cheese-cloth and absorbent cotton. The agar should be clear and of a yellowish straw color. Fill the test tubes, 10 c. c. to a tube, plug them with cotton and then sterilize them.

Gelatin.-To one liter of bouillon add 12 per cent of a good grade of gelatin. Weigh the dish and material. Dissolve the gelatin and titrate to 1 per cent acidity, boil 15 minutes, cool, add the white of an egg shaken with 30 c.c. of water, boil again for 15 minutes, restore weight, and filter through cheesecloth and absorbent cotton. Place in test tubes, 10 c. c. per tube, plug and sterilize.

Lactose gelatin.-Prepare as for gelatin in previous paragraph, using 15 per cent gelatin and 1 per cent lactose (milk sugar). In filling the tubes, use exactly 8 c. c., if litmus is to be added, otherwise 10 c.c. per tube; plug and sterilize as before.

Litmus.-Soak 100. grams of litmus cubes in 600 c. c. of water for 24 hours. Filter throngh filter paper and make up to original volume. Titrate the litmus solution so that the acidity will be the same as for media, viz.: +1 per cent. As it is too alka- 
line, titrate against one-twentieth normal hydrochloric acid, using litmus as its own indicator. (Litmus is blue when alkaline and red when acid.) The neutral point will be recognized by the blue solution turning reddish. If the neutral point of the solution using phenol phthalein as an indicator is zero, then the neutral point using litmus as an indicator would require 2.5 c. c. more acid. Therefore, in order to secure 1 per cent acidity to phenol phthalein, 5 c.c. of the litmus solution would require 1.5 c.c. of twentieth normal hydrochloric acid. As the litmus solution is always alkaline, it will require normal acid; the amount can be determined in the same way as in titrating media. Sterilize litmus the same as water.

Normal sodium hydroxid $(\mathrm{NaOH})$ and normal hydrochloric acid $(\mathrm{HCl})$. - These solutions had best be bought from dealers. To make twentieth normal solutions, one part of the normal is added to 19 parts of distilled water.

Sterilization.-In bacteriological work, it is necessary to have all glassware, utensils and media sterile, that is, absolutely free from organic life. It must be remembered that media, bottles, flasks, etc., must be kept plugged, otherwise they become contaminated. Therefore, if a flask or bottle of media or water is opened, it must be resterilized before using again. The methods of sterilizing vary for the materials to be sterilized.

Cleaning glassware.-All glassware should be thoroughly washed with some good soap or soap powder, rinsed in clear water, and allowed to drain. When dry, the test tubes, pipettes, flasks and graduated cylinders are ready to be plugged. With the forceps, 
pull off a piece of cotton of sufficient size to fit into the opening of the article to be plugged without danger of falling out or of sticking. Put the pipettes into glass tubing which has been cut into lengths which will hold the pipettes, then plug firmly both ends of the tube or case. After plugging, the articles are ready for sterilizing. Metal boxes holding a number of pipettes may be used in place of glass tubes; and when much plating is to be done, there is economy of work and time in the practice, but there is greater possibility of the pipettes becoming contaminated. Place them with the petri dishes and glass bottles in the hot-air sterilizer or oven. Tip the stoppers of the glass bottles back, to prevent exploding. In many laboratories, dilution bottles without glass stoppers are used, plugs of cotton being substituted. This custom permits the use of cheaper bottles. These should be kept at a temperature of $150^{\circ} \mathrm{C}$. for one hour, or until the cotton plugs are slightly browned.

Water.-It is convenient to sterilize water in the liter flasks. Fill the flasks three-fourths full and plug them with cotton. If an autoclave is available, sterilize water for one hour at 15 pounds pressure. Otherwise, place the flask in boiling water, or steam and hold for one and a half to two hours.

Bouillon, gelatin and agar.-Media can be. sterilized in an autoclave for 20 minutes at 10 pounds pressure, or the intermittent method may be used. In sterlizing by this method, the flasks or tubes of media are steamed or placed in boiling water for 20 minutes; then left at room temperature for 24 hours, and 
steamed again for 20 minutes after the material has reached the temperature of steam. The third day they are steamed again for 20 minutes.

Procedure for plating.-Wipe off the desk or table with 5 per cent carbolic acid solution. Shake thoroughly twenty-five times, the sample of milk. Unless the bacterial count of the sample is nearly known, it is well to make three dilutions, with two plates per dilution (six plates in all). The dilution should be large enough so that not more than one or two hundred colonies will develop on the petri dish. Thus, in plating fresh milk, a dilution of $1: 100,1: 200$ and $1: 1000$ may be used. To make the $1: 1000$ dilution, measure out with a sterile pipette (care should be taken in drawing out the pipette from the case that the point touches nothing but the milk, and, if many pipettes are kept in a metal case, each pipette should be passed through a flame before using it) 1 c. c. of milk, and put it in a sterile bottle containing 99 c. c. of the sterilized water. Shake this thoroughly. With another pipette, place 1 c. c. of the solution in a petri dish, being careful to raise the cover of the petri dish only high enough to introduce the mouth of the pipette. Five-tenths of a cubic centimeter of this solution would give a dilution of $1: 200$, while .1 would be $1: 1,000$. If higher dilutions are needed, more bottles may be used; for instance, if a dilution of $1: 1,000,000$ is wanted, arrange two bottles each with 99 c. c. of water. Place $1 \mathrm{c}$. c. of milk in the first, shake thoroughly, take 1 c. c. of this dilution and place in the second. This is then ready to transfer to the plate. 
Checks.-With a sterile pipette, place 1 c. c. of the dilution water in a sterile plate, and add the usual amount of media. Also pour 10 c. c. of media into a sterile, empty petri dish. If litmus is used, make a third check with 2 c. c. of litmus and 10 c. c. of the media.. In this way the state of the material may be determined. The following scheme from the Iowa Bacteriological Laboratory Report will be of value in estimating dilutions:

After the diluted milk is in the petri dish, if the litmus is wanted, add 2 c. c. with a sterile pipette. If not desired, this may be omitted. Melt the tubes of agar or gelatin, cool and maintain at a temperature of $40^{\circ} \mathrm{C}$. Finally, add $10 \mathrm{c}$. c. of the nutrient media, either agar or gelatin as desired, being careful to pass the mouth of the tube through a flame before pouring it. Give the dish a revolving motion, to mix the diluted milk and media, and then allow it to harden. Agar should be incubated at $37^{\circ} \mathrm{C}$. for forty-eight hours, while gelatin needs a temperature of $20^{\circ} \mathrm{C}$. for five days. When ready to count, place the petri dish over a counting-plate and, with a hand lens, count the number of colonies. If a counting-plate is not available, lines may be made across the bottom of the plate with a blue pencil, for marking glass to aid in counting. Each colony represents an original organism. Multiply the number of colonies by the dilution, and the result is the approximate number of organisms in the sample. Repeat this with six plates, and take the average of them as the final count.

GLASSWARE NECESSARY FOR PLATING ONE SAMPLE OF MILK

9 petri dishes.

31 c. c. (straight) pipettes.

12 c. c. pipette.

1 liter flask (water).

1250 c. c. flask (litmus).

2150 c. c. glass-stoppered bottles.
1250 c. c. glass-stoppered bottle.

1 graduated cylinder 100 c. c.

5 feet glass tubing, width $10 \mathrm{~m}$. $\mathrm{m}$. or metal box.

9 test tubes (Board of Health)

1 counting plate. 
GLASSWARE NECESSARY FOR MAKING ONE LITER OF MEDIA

1 thermometer (Centigrade 2 funnels, diameter, $15 \mathrm{c.m}$. scale).

1 flask (liter).

2 burettes -50 c. c. graduated 15 c. c. pipette. to $.1 \mathrm{c.c}$.

2 agate pails.

APPARATUS

Steam sterilizer.

Autoclave.

Hot air sterilizer.

Or,

Steam cooker or high altitude cooker and

Kitchen oven.

2 4-quart aluminium or agate kettles.

1 balances or scales (metric) sensitive to grams.

6 wire baskets.

1 hand lens.

1 Bunsen burner or alcohol lamp.

1 forceps.

MATERIALS

Beef extract (Liebig's).

Peptone (Wittes).

$\mathrm{NaOH}$ (normal).

$\mathrm{HCl}$ (normal).

Agar (threads).

Gelatine (Gold Label).

Lactose (pulverized).
Litmus (cubes).

Cotton (absorbent).

Cotton, common.

Phenol phthalein.

Alcohol.

Filter paper-diameter 45 c. m. 


\section{CHAPTER VII}

\section{MARKET MILK}

IN GENERAL, any conditions which make milk of better quality for manufacture, also make it of better quality to be consumed as milk. These conditions are not only those which have to do with the composition of the milk, but any other influences, as feed, health, care of the cows, or conditions of cleanliness. Several conditions affect the quality of milk intended for consumption as such. In the first place, it must be of high quality so far as the composition is concerned. Second, it must be secreted from healthy cows, fed on pure food and kept in clean stables. Third, it must be so treated that the fat does not readily separate from the other solids, and it must not readily undergo fermentation. Last of all, it must be clean.

Cleanliness.-Since cleanliness is equally important, whether the milk is intended for consumption or manufacture, it is well to take this up in detail first. All vessels used to contain milk should be heavily tinned; pails, cans, and the like, that are of the kind called ironclad are preferable on account of durability. An efficient means of attaining cleanliness is in avoiding seams in the utensils. 
This is secured by the use of the pressed or seamless vessels wherever possible, and when it is not possible to use these, by taking care that the joints are completely and smoothly filled with solder. In ordinary pails as found in the market this is never done, and it is a matter of considerable importance to the purchaser that all such seams be resoldered before using. The tinware should be kept bright and perfect. So soon as any rust spots make their appearance, an entrance is given into the soft iron for germs and small particles of decaying matter, which are in consequence removed with much more difficulty. Milk is much more easily removed from vessels when it has not been allowed to become dried upon their surface. If rinsed as soon as emptied, tin vessels may be much more easily cleaned than if allowed to stand for several hours. For such rinsing, lukewarm water is much preferable to cold or hot water. Cold water does not so readily unite with the milk as warm water, and hot water, by coagulating the albumin, may cause the milk to stick or "cook on" to the sides of the vessel. The process of cleaning vessels that have contained milk should be: First, to rinse them thoroughly in lukewarm water; second, to wash them thoroughly with the aid of some good soap or alkali, in water as hot as the hand will bear; third, to thoroughly rinse in hot water; fourth, to expose to live steam from one to two minutes; fifth, exposure, if possible, in bright sunlight from two to three hours. With these precautions, not only will the tinware be 
kept clean and bright, but no germs will find a resting place in the erevices. The use of much strong alkali is inadvisable, as it serves to cloud and tarnish the tin, giving it a dull appearance. If the vessels are exposed to steam until they are thoroughly hot, and then placed in such position that they will drain, no other drying will be necessary.

Having taken every precaution that the vessels are thoroughly cleansed, the prevention of access of dirt to the milk in process of milking is important. To this end, the body, especially the lower part of the belly and udder, of the cow should be thoroughly brushed and preferably dampened just before milking. The hands of the milker should be clean and his clothes free from dust, and the air of the stable should be free from dust. If the stable floor is dampened, it will not only aid in this respect, but in summer time will materially reduce the temperature of hot and overcrowded stables. With these precautions, we may expect to secure milk containing a minimum amount of dirt; * but with all these precautions, more or less dirt will find access to it. And immediately after milking, as much as possible of this dirt should be removed by at once straining the milk through a brass wire strainer of not less than fifty meshes to the inch and three or four thicknesses of loosely woven cotton or woolen cloth. The cloth strainer not only removes fine particles of dirt, but also

*These directions will serve to secure milk that is reasonably clean. So much importance is now placed on extreme cleanliness and there is so large a demand for the extremely high grades of milk known as "certified," that a chapter on certified milk has been added to this edition. 
entangles a considerable number of germs, and hence these strainers should be cleansed with great care, and should be frequently renewed.

Treatment after drawing.- So soon as the milk is drawn, it should be rapidly brought to a temperature slightly below the surrounding atmosphere. While it is being cooled it should be stirred to prevent the cream from rising, and in milk that has been cooled in this way there will be comparatively little tendency afterward for the cream to separate from the milk. Milk so treated is in an ideal condition for consumption, even though the consumer may consider the quality poor because of the slight tendency of the cream to form on the surface.

Aëration of milk. - Milk when drawn from the cow contains a certain amount of dissolved gases. These gases contain more or less

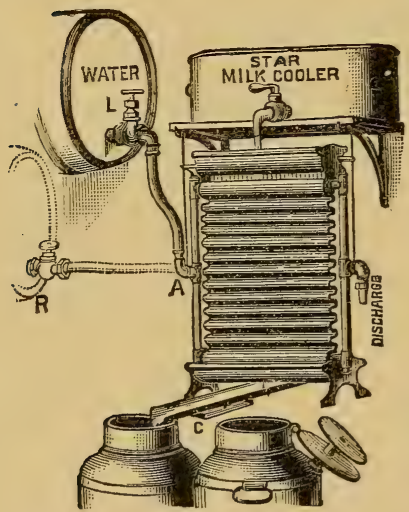

Fig. 16. "Star" milk cooler and aërator. of what is known as animal odor, the amount of this odor depending very largely upon the physical condition of the animal at the time the milk is drawn. Sometimes the amount is very slight and scarcely noticeable, at other times it is so great as to be extremely offensive. These gases and the accompanying odor are easily removed from the milk by exposure of the 
milk to the air during the process of cooling, and to this extent aëration of the milk is an advantage. Various forms of aërators and combined aërators and coolers have been devised, many of which are simple and effective, and the best results follow their use. In order to secure these results by aëration, however, it is necessary that the apparatus used for aëration should expose the milk thoroughly to the air, should not be cumbersome, and should be simple and easily cleaned; moreover, the process of aëration should always take place in the purest atmosphere possible.

Delivery of the milk. - In ordinary practice in the smaller towns and villages, and to a considerable extent in the larger cities also, the.

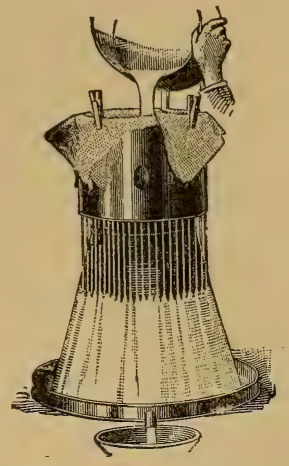

Fig. 17. "Champion" milk cooler and aërator. milk is placed in cans in which it is transported from the dairy, and is measured out in small quantities to each customer from the cans in which it was originally placed. Where care is taken to cool the milk, as described, and during the process of serving the customers to keep the contents of the cans well stirred, substantial justice is done each individual customer in the matter of giving him the due proportion of cream and skimmed milk. This has been well shown in a trial made at the Cornell University Agricultural Experiment Station* several years ago,

* Cornell University Agricultural Experiment Station, Bulletin No. 20. 
the interesting results of which are quoted below in full detail :

To determine just how much variation there is in the fat of milk served to the different patrons of a route by dipping, a member of the Station staff accompanied a milkman as he went upon his route, and as the milk was about to be served to various patrons, took samples for analysis. The dipper, such as is ordinarily used by milkmen, was provided with a long handle, so that it rested on the bottom of the ean when not in use. The milk was not stirred except by the motion of the wagon and the raising of the dipper. Twelve samples were taken, and yielded to analysis the following percentages of fat:

$\left.\begin{array}{rrrr}\text { No. } & 1 & . & 4.52 \\ \text { " } & 2 & 3 & 4.43 \\ \text { " } & 4 & 4.41\end{array}\right\} \quad$ Taken from Can A.

The milk was contained in four 30 -quart cans, marked A, B, C and D. The samples were taken as follows:

No. 1. - Taken from A at 5.50 a. m., within a few rods of starting.

No. 2.- Taken when the milk in A was half gone, at 6.10. Seventeen dips had been made since No. 1 was taken, and three-fourths of a mile traveled.

No. 3. - Taken from the bottom of $\mathrm{A}$ at 6.20. Twelve dips had been made since No. 2 was taken, and three-fourths of a mile traveled.

No. 4. - Taken from the top of B at 6.10, three-fourths of a mile from starting. 
No. 5. - Taken from the middle of $B$ at 7.20. Six quarts had been added to $B$ at 6.35 , and two and one-fourth miles traveled between taking samples 4 and 5 .

No. 6. - Taken from the bottom of $B$ at 7.55. One and three-quarter miles traveled since taking sample 5.

No. 7. - Taken, from the top of $\mathrm{C}$ at 6.20 , one and onehalf miles from the start.

No. 8. - Taken from the middle of $\mathrm{C}$ at 6.50. One mile had been traveled since taking No. 7. At 6.35 six quarts remaining in the bottom of $\mathrm{A}$, and about an equal quantity bought of another dealer, had been added to $\mathrm{C}$.

No. 9.-Taken from the bottom of $\mathrm{C}$ at 7.00. Fifteen dips had been made, and a half mile traveled, since 8 was taken.

No. 10. - Taken from the top of D at 7.50.

No. 11. - Taken from the middle of $D$ at a time when the wagon had stood still for four minutes, with the dipper resting on the bottom of the can. Time, 8.15, and one mile traveled since 10 was taken.

No. 12. - Taken from the bottom of D.

A second and third trial gave similar results.

\begin{tabular}{|c|c|c|c|c|c|}
\hline & & & ECOND & TRIAL. & \\
\hline 1 & & $\begin{array}{c}\text { a. } \\
.4 .86\end{array}$ & $\begin{array}{l}\mathrm{b} . \\
4.78\end{array}$ & $\begin{array}{c}\text { Average. } \\
4.82\end{array}$ & (Before starting.) \\
\hline Sample 2 & & .4 .71 & & 4.71 & (Top of can.) \\
\hline Sample 3 & & .4 .82 & & 4.82 & (One-third gone.) \\
\hline Sample 4 & & .4 .83 & 4.74 & 4.78 & (Two-thirds gone.) \\
\hline Sample 5 & & .4 .73 & 4.82 & 4.77 & (Bottom of can.) \\
\hline & & & THIRD & TRIAL. & \\
\hline & & & b. & Average. & \\
\hline $\begin{array}{l}\text { Sample } 1 \\
\text { Sample } 2\end{array}$ & & $\begin{array}{l}.4 .20 \\
. \quad 4.11\end{array}$ & $\begin{array}{l}4.16 \\
4.00\end{array}$ & $\begin{array}{l}4.18 \\
4.05\end{array}$ & (One-fourth gone.) \\
\hline Sample 3 & & 4.13 & 4.01 & 4.07 & (One-half gone.) \\
\hline Sample 4 & & .4 .15 & 4.04 & 4.09 & (Three-fourths gone) \\
\hline Sample 5 & . &. .4 .01 & 4.00 & 4.00 & (Bottom of can.) \\
\hline
\end{tabular}


These results were abundantly confirmed by a similar series made by Dean in Canada.*

The removal of the covers of the cans in the dusty and dirty streets always results in considerable contamination of the milk, hence the practice of putting the

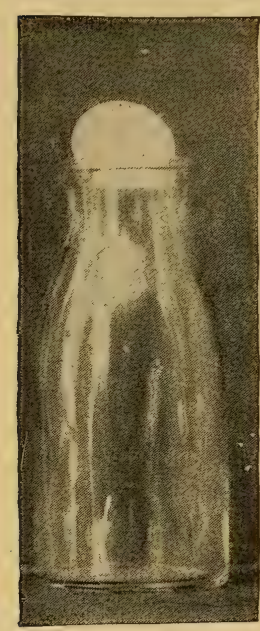

Fig. 18. "Common Sense" milk shipping bottle. milk into bottles upon the farm and delivering these bottles intact to the consumer, has rapidly increased since its introduction, some ten years ago, and is now in almost universal use. When the bottles are used, the milk should be put into them as soon as it is drawn, strained and cooled; they should then be sealed and kept in a cool place until ready for delivery. This method of delivery, although it entails a greater expense in outfit and transportation and a considerable loss from breakage, is much to be preferred to the old manner.

Milk so handled, and kept at a temperature between $45^{\circ}$ and $50^{\circ}$ F., should be in good condition sixty hours after it is drawn. Its life can be prolonged by pasteurization, and the liability to transmission of diseases through the milk at the same time reduced to a minimum, but whether pasteurization should be relied upon for these purposes is still somewhat of an open question. In so far as immunity from diseases which may be present in the

\footnotetext{
* Ontario Agricultural College, Bulletin No, 66.
} 
cows is concerned, official inspection of the herds is undoubtedly a greater safeguard than dependence upon pasteurization or sterilization; and so far as the liability of transmission of other diseases is concerned, the milkman who is careless in regard to the cleansing of his utensils would quite as likely be careless in the pasteurization or sterilization process, so that reliability of the milkman is an important factor in the purity of the milk supply, no matter what other precautions are taken.

Bad flavors in milk.-Milk may be unfit for consumption for reasons other than the presence of dirt or infection with bacterial germs. The cow herself is often responsible for bad flavors in milk. When lactation is far advanced the milk often has a disagreeable salty taste. In extreme cases it may even be described as acrid or bitter. While there is nothing particularly unwholesome about such milk, its bad flavor makes it unfit for food, and if the cow is within two months of calving, she should be allowed to go dry at once. If the time before calving is longer than this, the bad flavor may often be remedied by taking care that the cow has plenty of succulent food, as roots or silage, and particularly that the proportion of dry, fibrous food is reduced to a minimum.

Digestive disorders of any sort in the cow are frequently accompanied by strong flavors in the milk. These flavors are not to be attributed to the food, but to the bad condition of the animal, and they normally tend to disappear when the digestive 
organs regain their tone. Disorders of this sort are especially likely to occur when cows that have been poorly fed during the winter are suddenly turned on fresh rank pasture in the spring.

Quality of milk for consumption.-The fat is the most variable and the most valuable constituent of the milk, so that milk is ordinarily considered to be of value for human food in proportion to the amount of fat it contains, but where it is to be used as a food in large quantities, the fat may frequently be present in too large quantities for the digestion of many persons. Milk containing about 4 per cent of fat is probably an ideal food for the general mass of human beings. If there is above 5 per cent of fat the other solids are somewhat out of proportion, and many are likely to have trouble with their digestion from using large amounts of such milk. On the other hand, if there is less than 3 per cent of fat, the casein and other solids are in too great proportion to the fat, and are less readily digested. The question of the healthfulness of milk from which a large part of the fat has been removed is one frequently discussed. The removal of the fat does not thereby in any way injure the other solids; they are still there, and still as useful for food as before the fat was removed, only in consuming such milk as food the fat must be supplied in some other way. For the person of vigorous digestion, who for reasons of economy desires to supply the fat required by his system in some cheaper form than that of milk fat, skimmed milk is 
a wholesome and nutritious article of diet, which furnishes to the system. almost the same sort of nutrients that lean meat, eggs, or foods of like nature do, and no one should be debarred by legislative or municipal enactment from using such an article of food if he so desires.

Control of milk supply.-Since milk is so extremely variable in quality, and is so easily adulterated; since often considerable variations are not readily detected; above all, because the amount of milk used by any given person or family is comparatively small, the consumer of milk is almost wholly at the merey of the producer and dealer, and must rely for a good product very largely upon their honor. The state has recognized this, and to prevent imposition by unscrupulous people, has in various ways sought to regulate the sale of milk and like products. The chief means used has been to establish arbitrary standards of quality, and to subject to fine those dealers whose goods should be found to be below the required standard. The standards established by various states and municipalities have varied widely. From 2.5 to 3.7 per cent of fat, and from 11.5 to 13 per cent of total solids, have been the minimum requirements. (See Appendix C.) These standards, while efficient in securing honest dealing where they are rigidly enforced, nevertheless may work injustice, so far as the honesty of the dealer is concerned, under various circumstances, and may prevent the production and sale of a comparatively low quality product at a reasonable price. It 
would seem, therefore, that the best means of regulating the traffic in milk would be, not to set up an artificial standard to which all must come, but to require each individual dealer to guarantee his own standard, and hold him responsible if his milk were found below. In this way it would be possible to sell milk of various qualities, from strictly skimmed to heavy cream, upon a graduated scale of prices, with exact justice to every one.

Cream for consumption. - Since the introduction of the centrifugal separator, the use of cream as an article of diet and for household purposes has very rapidly increased, and the amount of cream so used now represents a very considerable proportion of the total production of milk. The sale of cream to consumers is usually carried on in connection with the sale of milk, and the conditions of care and cleanliness necessary in the one case apply equally well to the other. Cream, however, is much more generally delivered in bottles than is milk, and since the daily quantity used is smaller, there is a greater demand that it should keep sweet for a longer time. For this reason it is still more important that eream should be kept, so far as possible, free from contamination with germs of fermentation, and at a comparatively low temperature from the time it leaves the separator till it goes into the consumer's hands; and of course the fresher and freer from germs the milk is when separated, the better will be the keeping qualities of the resulting cream. Milk containing more than .2 per cent of lactic acid should not be used for the pro- 
duction of cream for commercial purposes. With care it is not difficult to produce cream that will remain sweet for four or five days or even a week.

Pasteurized cream.-For the sake of its better keeping qualities cream that is to be used for commercial purposes is often pasteurized. If it is pasteurized at $155^{\circ} \mathrm{F}$. for 10 minutes and quickly cooled to $50^{\circ} \mathrm{F}$. or below, and bottled in sterile bottles, it will keep, with ordinary precautions, for a week or more. Cream so pasteurized will have no perceptibly cooked taste, but it will be considerably thinner in consistency than cream of a like percentage of fat that has not been pasteurized, because the pasteurization greatly and permanently reduces the viscosity. Owing to the fact that the "quality" or richness of the cream in fat is, in popular estimation, almost wholly in proportion to its consistency, this lack of consistency in pasteurized cream is a matter of considerable commercial importance. Babcock and Russel* have shown that the consistency may be restored by the addition of a small amount of a solution of lime in cane sugar, to which they have given the name viscogen. The amount added is so small (about 1 part to 150 of cream) that, while the consistency is perfectly restored, the cream is not affected in odor, taste or composition; but since the addition of anything whatever to milk or cream is prohibited in many states, cream to which viscogen has been added should always be sold under a distinctive name, as visco-cream. For preparation of viscogen, see Appendix A.

\footnotetext{
*Wisconsin Agricultural Experiment Station, 13th Report, p. 81.
} 
Quality of cream.-The most unsatisfactory thing about the sale of cream, commercially, is its varying percentage of fat. Since cream is merely milk into which a greater or less proportion of fat has been gathered, it follows that cream may be anything that the seller can induce the puchaser to accept under that name, and may contain anywhere from 6 or 8 up to 60 or 70 per cent of fat. Where cream is raised by the gravity process it will contain from 18 to 22 per cent of fat; but where it is separated by centrifugal process the separator can be so adjusted as to take cream of almost any fat content desired.

A moderately heavy cream is quite as useful and desirable for table and domestic purposes as one excessively rich in butter-fat. The United States Department of Agriculture has recommended a minimum standard of 18 per cent fat for cream for commercial purposes. Such a cream is rich enough for most table and domestic use, and is one that can easily be produced by a gravity process. Very many state laws have recognized the federal standard, though other standards are also in force (see Appendix C). 


\section{CHAPTER VIII}

\section{CERTIFIED MILK}

Definition.-Commercial certified milk is a comparatively new product in this country, and, strictly speaking, includes milk the qualities of which, especially those that depend upon strict sanitary precautions, are certified to or guaranteed by some official organization, usually a milk commission, appointed by a medical society or a board of health. Such commissions maintain a somewhat strict supervision over the production and handling of the milk, and certify to its quality as to cleanliness, purity and composition. This guarantee, or certification, gives the consumer a fairly satisfactory assurance that he receives milk of superior quality. In a somewhat broader sense, the term certified milk may include milk of like quality produced with equal care, although it may not be certified to by any official organization. Originally the term certified milk was copyrighted, and rightly used only by a medical society in New Jersey that first certified to milk in the United States.

Origin of certified milk.-Primarily, certified milk was produced for infant-feeding and for hospital use. The high mortality of infants in the great cities led boards of health to seek for a purer and more 
wholesome product for this particular purpose. The expense of producing milk of this particular grade is necessarily great, as the labor involved is very much more than that required to produce ordinary market milk, and the continual oversight by trained men unavoidably increases the cost of production very materially. Certified milk differs from pasteurized or sterilized milk in this important essential. The former is kept as clean and as free from foreign matter as possible, while the latter is treated usually to some degree of heat, to kill and prevent the growth of objectionable bacteria. Perhaps the most ideal condition for the consumption of milk is secured when the milk is drawn by the young directly from the mammary glands of the mother. However, when the dairyman attempts to serve his customers in the great cities, hundreds of miles from the source of production, with a like quality of milk, many difficulties are encountered, some of which are discussed in the following pages. It is the aim of the producer of certified milk to approach as nearly as possible the ideal condition above mentioned by excluding all foreign matter and by keeping the milk at a low temperature.

Certified milk has been kept for months in a perfectly sweet condition. It has been sent from the interior of this continent to Europe, and returned without any indication of souring. After such a journey or length of time, it would not, however, be considered as safe for food as the fresh product, even though it had not soured to any perceptible degree. 
Standards. - The commissions that are now supervising the production of certified milk have established various standards as to composition and bacterial content. Most of these standards exclude from certification milk that has a general bacterial content of more than from 10,000 to 30,000 per cubic centimeter, and the requirement as to fat-content is usually somewhat more than 4 per cent. It is not at all uncommon for careful producers to secure milk having but a few hundred bacteria per cubic centimeter.

Production.-In the production of certified milk, it is quite essential that the stables be constructed with particular reference to cleanliness. As the labor involved is one of the most important factors in the expense of production, the ease with which the stable may be kept clean becomes an important factor. As wood was formerly the cheapest of the building niaterials in this country, it was used largely and, in many cases, almost exclusively, in the construction of stables; but, as the certified milk producer desires the most sanitary and at the same time the most durable structure, he has eliminated wood as a building material to a large degree. While glazed tile and glazed brick make a most ideal structure, ret their expense in most cases prevents their use in the construction of stables that are to prove financially a paying investment. Modern methods of cenuent construction are proving quite satisfactory. The floors, walls, and even the roof, are sometimes constructed of this material and seem to meet, fairly well, the requirements of a stable for the production of certified milk. Mod- 
ern methods of waterproofing the stable floors have overcome quite largely the objections that were formerly made to cement because it was damp, and consequently a great conductor of heat.

It is important that the stables be constructed with particular reference to ventilation. It is essential that the air in the stable shall be comparatively fresh at all times. While there is no accepted standard or easy means of determining the purity of the air in the stable, yet it may be said that it will be sufficiently pure when, on entering the stable from out-of-doors, the air presents a fair degree of freshness, with no marked animal odors or perceptible staleness. If such odor or staleness is obvious, the stable should be considered not sufficiently well ventilated.

As dust is a great carrier of bacteria, it is important to admit only pure air and to have all dairy-rooms and stables as free from dust as possible. When conditions will permit, it will be found an advantage to have as much as possible of the grounds surrounding the stable covered with turf. This will prevent, to a considerable degree, the entrance of dust in the stable, particularly during the dry summer months when it is most difficult to produce milk having a low bacterial content.

Sanitary stables. - It is important that sunlight be permitted to enter the stables. This feature is considered of so much importance by some milk producers that they endeavor to arrange their stables so that the sunlight may be permitted to enter on one side of the stable in the forenoon and on the other side in 
the afternoon. This is most desirable during the winter months, in order to give as much direct sunlight as possible to the stables, because of its effect in promoting the general health of the cattle, as well as in destroying or retarding the development of bacteria.

The amount of window surface in the cow-stable should at least be sufficient to provide four square feet of clear window surface per cow. If this amount, or more, is provided, and the windows are fairly well distributed, the stable will be sufficiently well lighted for all practical purposes. While some may desire more than this, yet, if the building is so situated that the windows will admit direct sunlight, the window surface stated will be quite sufficient.

In constructing a stable, it should be made sufficiently large to give each animal at least 600 cubic feet of air space. If a less amount is provided, it will be found more difficult to keep the air pure without causing perceptible drafts of air produced by ventilation. The ideal ventilation secures a gradual change without producing drafts, and yet sufficient to maintain the desired degree of freshness.

The ease with which the stable may be cleaned is an important consideration. Not only should it be so constructed that it is easy to remove the voidings of the animals, but it is necessary to prevent accumulations of dust in any part of the stable, as well. It is, of course, impossible to so construct a practical stable that dust will not settle in some places. This, however, should be removed frequently, so there shall be no accumulation. 
Selection of the cows. - It is important that none but sound, healthy cows be selected for the production of certified milk. The strongest and most vigorous organizations not only produce milk best in quality, but are most certain to stand the strain incident to the trials of heavy dairy feeding. It is also important from a financial standpoint that none but heavy milkers be selected, as the labor of caring for the animals is so great as to make light milkers even more unprofitable in the certified milk stable than on the ordinary farm. Sufficient care should be exercised to select cows that have sound and normal udders. Those that have at some time been affected with garget, even though apparently fully recovered, should be regarded with suspicion. It is well understood that some cows, although apparently in a perfectly normal, healthy condition, produce milk having a somewhat high bacterial content. While these cows are not so desirable, yet it is impossible to weed them out without making one or more bacterial examinations of the milk of each individual. Cows that show any indication of irritation at milking time are not desirable. Any unnecessary movement tends to produce more or less dust, particularly in the winter time, when the stable floors'are partially or wholly covered with litter.

Provision should be made to remove all cows from the certified milk barn that have reached the period of lactation when they are known as strippers. On the other hand, they should not be placed in the certified herd until they are surely over the trials of par- 
turition. Ten days from calving should usually elapse before using the milk as certified.

Care of the cows. - It is customary to clip the hair from a considerable portion of the rear of the cow, particularly from the udder, flank, tail, thighs, etc., to facilitate the ease of keeping them clean. Anything that will tend to promote the ease in keeping the cows or the stable clean and, at the same time, not detract from the comfort of the animal, will aid materially in the production of pure milk. In order to prevent the brushing of dust or dirt from the cows by the milkers at milking time, the cows should be thoroughly groomed each day, and lightly groomed or thoroughly brushed before each milking. It should constantly be borne in mind that dust and all fine particles of dirt carry large numbers of bacteria, and that the most successful producer of clean milk devises means by which these undesirable foreign products may be most perfectly excluded. Consequently, the cows should not be fed just before milking time any food that is liable to cause any dust or disagreeable or objectionable odors in the stable. The time for feeding the dry, coarse fodders is immediately after milking, so that the stable may be most effectually free from the dust incident to feeding. It is important, also, to use litter or bedding that will make as little dust as possible. Clean, dry pine shavings are popular on this account. While fine-cut wheat or rye straw may be used, yet the shavings are preferred by most dairymen on account of cleanliness and ease of handling. 
Care of the stable.-The thorough cleansing of the stable is an important factor in the production of clean milk. Not only should the stable be thoroughly cleaned of the voidings, but it should be washed carefully every day. If the cows are kept in the stable most of the time, it should be cleaned of voidings two or three times daily. The thorough cleaning of the stable is an important aid in keeping the stable air comparatively fresh. An abundant supply of water, under pressure, that may be forced through a hose, is, of course, very desirable. If the water supply is somewhat limited, or the use of a hose not permitted, the use of disinfectants is oftentimes resorted to, and undoubtedly is a very great aid in maintaining a low bacterial content in the milk. However, the use of disinfectants cannot take the place of thorough cleansing, as the disinfectants will not prevent dust from accumulating. Before each milking, the stable should be freed from dust as perfectly as possible. Various means are employed to accomplish this end. Where water is at hand, under sufficient pressure to produce a fine spray, spraying the whole interior of the stable is an effective means of allaying the dust. When this cannot be done, some snccessful dairymen turn live steam into the stable in sufficient amounts to perfectly clear the stable of all dust. As this steam condenses, and falls, it carries the particles of dust with it. This, as does spraying, tends to dampen the litter and those parts of the cows that are not washed, and thus prevents dust by any movement of the cows. 
Milking.-In the production of certified milk, cleanliness all along the line is most essential, and the cleanliness of the milkers and their wearing apparel is quite important. The milker should be scrupulously clean, and should wear clean clothing, preferably cotton suits that will readily show any accumulation of dirt. Each milker should be provided with a clean suit as often as every day. After the dust is removed from the stable by spraying, or by the other means described above, before the milking commences, the cows should be thoroughly washed on those parts that are likely to come in contact with the milker, such as the sides, thighs, udder, tail, etc. These parts should be washed in at least two waters, to the first of which should be added some odorless disinfectant. After the last washing, the parts should be wiped with a damp cloth so there is no danger of dripping. Then the cows are ready to be milked. Practically without exception, the milkers in a certified barn use some kind of covered pail. There are several kinds of covered pails with various-sized openings on the market, from which choice may be made. They are, however, pretty well agreed that milking through a strainer is not to be commended. It undoubtedly is a most excellent practice to strain the milk of each cow separately. This gives the dairyman an opportunity to reject the milk from any cow that does not seem to be quite normal. Occasionally an individual produces a little slightly stringy milk that would not be detected by the milker, but can be rejected at straining time if the milk from each cow is strained separately. 

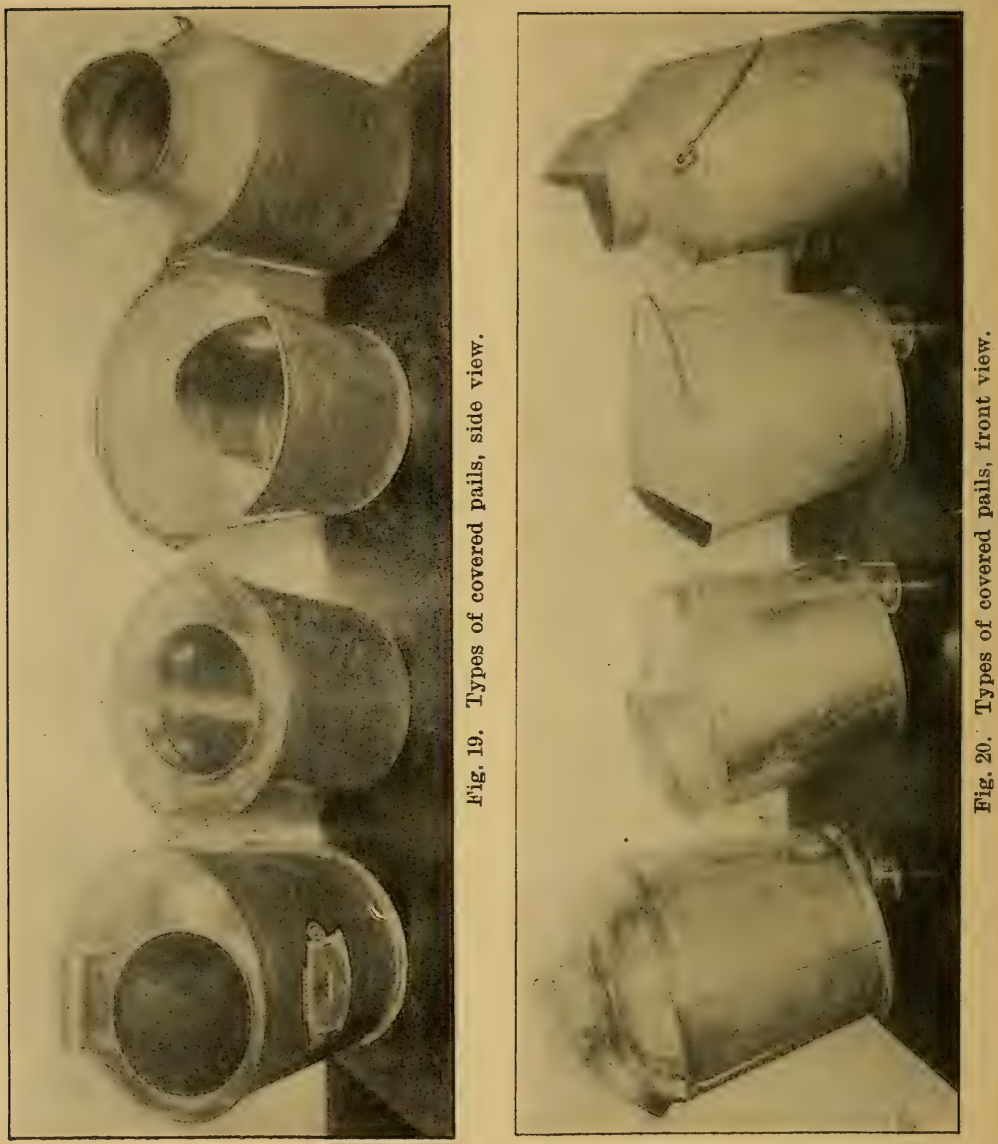
The milk should be removed from the stable immediately after it is drawn. It is needless to say that the milking should always be done with dry hands. After carrying the milk of one cow from the stable, the milker should thoroughly wash and wipe his hands before returning to milk another.

It is equally important that thorough cleanliness of both vessels and air be maintained when the milk is removed from one vessel to another. The question of providing pure air that is free from dust for the straining-, cooling- and bottling-rooms, is sometimes a difficult one to solve. During the winter months, when the ground is covered with snow, it is not so difficult; but when, during the summer, the air is more or less dust-laden, it is not easy to free it from dust before admitting it to the milk-room. Where large quantities of milk are handled in close proximity to the sterilizing apparatus, the air becomes heated and requires frequent changing, so that it is difficult to ventilate and admit none but pure air. It is very much better to admit air to the cooling- and bottlingrooms through a flue of a considerable height, as the air near the ground is more likely to be dust-laden than that higher up. When an abundance of water nnder pressure is at hand, an effective and most satisfactory means of ventilating is to force the air down a flue and into the room by means of one or more fine sprays from an ordinary fine-spray nozzle, placed at or near the top of the flue. This not only drives the air into the room, but it removes the dust before it enters. One of the most successful dairymen, who 
has not the advantage of water pressure, pumps the water to the top of a flue, and permits it to run through small apertures and drop like rain, thus eausing a current of air down the flue and, at the same time, freeing it from dust.

Milk should be cooled as soon as possible after it is drawn from the cow, and the temperature should not be allowed to rise until it is in the consumers' hands. While every precaution should be taken to exclude dust or dirt of any kind from the milk, it is yet necessary to strain the milk before cooling through sterilized strainers of cotton wool.

Immediately after cooling, it should be bottled, and the bottles packed immediately in the receptacle in which they are shipped. When the milk is to be shipped considerable distances, it is customary to protect the cap with which the bottle is sealed with either paraffin or some sort of lead foil, paper, or tin caps. Some of these caps are attached to the bottles with lead seals, so that it is impossible to remove the contents without breaking the seals. Certified milk bottles are usually packed in cases carrying twelve quarts, and are sufficiently large to hold enough crushed ice to withstand a shipment of three or four hundred miles.

Care of utensils. - It is necessary to exercise the greatest care in washing the bottles and preparing them for filling They should be thoronghly washed with water containing soap or cleansing powder, and then rinsed, preferably by an arrangement that will permit each bottle to be rinsed with pure water that does not come in contact with any other bottle. After 
rinsing, they should be drained, placed mouth downward, and sterilized, preferably with steam under a few pounds pressure. There are some machines on the market that will fill the bottles and cap them without the attendant handling either bottles or caps. When the bottles and caps are both sterile, this, of course, is a somewhat desirable feature. There is no reason whatever why just as good results may not be obtained in smaller plants, if the operator desires to fill the bottles by means of a pitcher or dipper or some other convenient vessel, and, as a matter of fact, this method has been employed by some very successful of the smaller producers. In certified milk plants, where large quantities of milk are handled, some of the modern bottle-filling machines prove quite satisfactory. Some dairymen set the bottles in ice-water as soon as they are filled and capped, in order to secure the quickest cooling. Others prefer to place the bottles in the shipping cases and cover with finely crushed ice.

All vessels that are used in the handling of milk should be thoroughly washed, rinsed, and sterilized, preferably by steam under pressure. In sterilizing cans and pails, it is quite important to have them placed in the sterilizer mouth downward.

Cost of production. - The cost of production of certified milk will depend quite largely on the location and arrangement of the barns, stables, bottling-rooms, etc., as well as the ease and convenience by means of which suitable roughage and concentrates may be secured and handled. It is also difficult to secure efficient labor for the cleaning and milking, and to 
keep them profitably employed during the whole day. As milk is usually packed in ice for shipment, easy access to a shipping-station becomes an important factor. Since for every pound of milk shipped there will be at least three pounds of extra weight in ice, bottles and cases, the breakage of bottles also adds materially to the cost. Under present conditions, the cost of producing and delivering certified milk at the shipping-station may be estimated, from a conservative standpoint, to be at least twice that of good market milk.

The various commissions that superintend the production and marketing of certified milk as yet have not agreed upon and adopted a uniform standard; consequently there is considerable variation as to the requirements, and some dissatisfaction among the producers of certified milk at what seem to them to be somewhat arbitrary rulings. This feature has undoubtedly deterred some from entering this field of production who otherwise might have done so had they been permitted to have complete control of their business.

The high cost of certified milk is undoubtedly the chief factor in preventing its more general use. There are, however, many people in our cities who desire milk of this grade, but, as they are pretty well scattered over the residence districts, the cost of delivery, as well as the cost of production, compared to that of ordinary market milk, seems to many to be excessively high. At the present time, under the existing requirements, the demand for certified milk in the eities of the United States seems to be fairly well provided for. 


\section{CHAPTER IX}

\section{SEPARATION OF CREAM}

CREAM is that part of milk into which a large portion of its fat has been gathered. It is composed of the same constituents as milk, but they are not in the same or any constant relative proportion. Cream is separated from milk to be consumed as food, and, as a matter of convenience, in the manufacture of butter. The separation of cream is always attended with some loss of fat. The percentage of fat in cream may vary anywhere between 8 and 70 per cent. Cream of good quality for commercial purposes should contain from 18 to 25 per cent of fat, and very rich cream contains from 35 to 40 per cent of fat. Cream is composed of globules of fat, with such part of the water and solids as adhere to them. Its separation from the milk is effected by means of the difference in specific gravity between the globules of fat and the milk serum. The fat in the milk is in the condition known as an emulsion; that is, in the form of minute globules, which are kept from running together and coalescing by means of the surface tension of their particles and the viscosity of the liquid in which they float; therefore, any condition of the milk 
which tends to increase the surface tension or the viscosity will act as a hindrance towards the separation of the cream. While the viscosity of the milk serum prevents the particles of fat from uniting into a mass, still the particles have considerable freedom of movement in the milk, and being of a less specific gravity, of course are acted upon with less intensity by any force to which the milk is subjected.

If the milk is allowed to remain at rest in a vessel, the force of gravity, acting with different intensities upon the globules of fat and the milk serum, will cause the particles of fat to gather together near the surface of the liquid. In so gathering, they carry with them certain of the milk constituents, and the layer of fat globules and adhering particles we call cream. From time immemorial, and until within a very recent date, the force of gravity, acting in the way indicated, has been the only means used for separating cream from milk. Now machines are in use that effect a separation of the cream from milk by means of centrifugal force, and at the present time we have three systems of separating cream; namely, by gravity acting upon a thin layer of milk in a shallow vessel, known as the Shallow Pan System; secondly, by gravity acting upon a deeper mass of milk, usually submerged or partially submerged in water, known as the Deep Setting System; and thirdly, by machines making use of centrifugal force, known as the Centrifugal or Separator System. The quality of the cream for consumption or for purposes of 
manufacture is not affected either one way or the other by any of these three systems, so that their relative economy rests wholly upon the completeness, cost and ease of separation.

Since the separation of cream from milk is always attended with some loss of fat, it is pertinent to inquire as to the necessity of any separation of the cream when butter is to be made. It is perfectly possible to manufacture butter directly, by churning whole milk without separating cream, and undoubtedly the first churns were skins of animals, into which the whole milk was placed and then agitated until the butter was brought; but under good conditions it is not possible to so completely remove the fat from the milk by a churning process as by a creaming process; so that while there is some loss in separating the cream, there is usually a greater loss in churning the butter from the whole milk. Even when the loss of fat in the buttermilk is no greater than the loss of fat in the skimmed milk, the greater amount of labor required to churn the whole mass of milk still renders creaming an economical practice in the manufacture of butter.

Gravity creaming.-In separating cream by force of gravity, there is a greater loss of fat, a longer time required, and the various conditions affecting the milk have a greater influence upon the creaming than when centrifugal separation is used. The conditions of the milk that affect the creaming by the gravity process are: First, the size of the fat 
globules; second, the amount of solids not fat in the milk; third, the character of the solids not fat. The larger the fat globules the more readily they separate from the milk, since the larger the sphere the less the ratio between the surface and the mass, so that a large fat globule meets with proportionately less resistance because of the viscosity of the milk than a small one. The size of the fat globules is to a great extent a characteristic of the breed and individuality of the animal, but cows newly calved secrete larger fat globules than those in advanced periods of lactation; consequently we find that the milk from new milch cows is more readily creamed than from those long in milk. The amount of solids not fat affects creaming by the gravity process because of the difference it effects in the specific gravity of the fat and milk serum. The solids not fat are all heavier than water, and in the milk are in a state of solution or semi-solution; hence, an increase in the amount of solids not fat increases the specific gravity of the milk serum in which they are dissolved, and makes the difference between the specific gravity of the fat and milk serum greater and the separation of the fat easier. The proportion of solids not fat is influenced by the period of lactation and, to a considerable extent, by the character of the food. Cows far advanced in lactation often give milk extremely rich in solids not fat. Cows fed on dry food give milk containing less water than those fed on watery or succulent foods. In so far as these conditions 
increase the amount of solids not fat, we shonld expect such milk to be more easily creamed, but the favorable effect of the increase of solids is more than counterbalanced by the unfavorable effect of the character of the solids. Of the solids not fat, albumin, casein, sugar and ash increase the viscosity of the milk in the order named, and of these the casein is more subject to variation, so that the increase in the proportion of solids not fat ordinarily means an increase in casein, and this means a largely increased viscosity. And the increase in viscosity tends to prevent the separation of the fat more than the increase in specific gravity tends to aid it. In general, the conditions which bring about an increase in the viscosity of the milk occur at the same time as the fat globules are growing smaller, so that we find a wide range of variability in the ease and completeness with which cream may be separated by gravity process.

Shallow pan creaming.-This is the oldest method of separating cream from milk, and notwithstanding the rapid changes that have taken place in creaming methods since the introduction of centrifugal separators, large amounts of butter are still made from cream separated in this way. The conditions most favorable for a complete separation of the fat in the shallow pan system are, that the milk should be put at rest in the pans as quickly as possible after it is drawn, that it should cool with a fair degree of rapidity to a temperature of $60^{\circ} \mathrm{F}$., and that it should remain as nearly as may be constantly at 
that temperature for at least thirty-six hours. Further, since the milk must remain for so long a time, it is essential that the atmosphere to which it is exposed be pure and free from currents of air and particles of dust. These conditions can be secured in a clean, cool, well ventilated cellar. In the shallow pan system, the depth of milk should be from 2 to 4 inches. Occasionally water, usually running water, is used to secure a quick cooling and more even temperature surrounding the milk, and when this is done the depth of the milk may be increased to 4 or 6 inches. Under the shallow pan system, the cream is separated from the milk by removing it from the surface with a thin flat instrument, usually made of tin, and called a skimmer. In this method of removal, it is not possible to completely remove all the cream that has risen to the surface, and in removing the cream more or less of the milk is taken with it, so that in the process of skimming there is a considerable loss of fat and a thin cream is always obtained; but even if the losses in the skimming operation could be obviated, the separation of the particles of fat in the shallow pan system is less complete than in either of the others. Under ordinarily good conditions, 20 per cent of the fat in the milk is lost when the shallow pan system is used. Skimmed milk containing less than .5 of 1 per cent of fat is rarely obtained under this system.

Deep setting system.-About thirty years ago it was discovered that if milk could be set in vessels when first drawn, and rapidly cooled to a tempera- 
ture of about $40^{\circ} \mathrm{F}$, and held at that temperature for twelve to twenty-four hours, not only could the depth be increased from 4 to 20 inches, but the separation was much more complete in a shorter time. In order to bring about quick cooling, vessels not more than a foot in diameter were used, and water, either from cold springs or containing ice, was used as the refrigerant. Two essentials, then, for complete creaming by this system are the rapid and immediate cooling of the milk to $40^{\circ} \mathrm{F}$., and a sufficient supply of ice to maintain this temperature for twenty-four hours. Under these conditions the fat may be so completely separated that not more than .2 of 1 per cent of fat is left in the skimmed milk. Various devices have been used, the best known of which is the Cooley system, in which the cans are not only surrounded by icecold water, but completely submerged in it, the chief effect of the submerging being to guard the milk against contamination through the atmosphere. The same conditions as to the character of the milk affect completeness of separation in both the shallow pan and deep setting systems, but no completely satisfactory explanation has ever been assigned as a reason why the fat globules should rise more rapidly and more completely through 20 inches of milk at a temperature of $40^{\circ} \mathrm{F}$. in the deep setting system, than through 4 inches at a temperature of $60^{\circ}-70^{\circ} \mathrm{F}$. in the shallow pan system.

It has been asserted that because water is a better conductor of heat than fat, it will cool more rapidly than the fat, and will increase in density 
till its maximum density is reached at about $40^{\circ} \mathrm{F}$., at which temperature the difference between the specific gravity of the water or milk serum and the fat will be at its greatest, and the separation of the fat for that reason promoted. But while the water does cool faster than the fat, the fat shrinks or increases in density much faster than the water, so that the difference in specific gravity between the two is no greater, and in fact less, at low temperatures than at high ones. Further, the viscosity of the milk serum is much increased at low temperatures, so that the favorable influence of low temperatures cannot be explained upon these grounds. Arnold* attempted to explain the favorable influence wholly upon the relative contraction of the fat and serum; as follows :

Water is a better conductor of heat than fat; hence when the temperature of milk varies either up or down, the water in the milk feels the effect of heat or cold sooner than the fat in the cream does, therefore the cream is always a little behind the water in swelling with heat or shrinking with cold, thus diminishing the difference between the specific gravity of the milk and cream when the temperature is rising, and increasing it when the temperature is falling.

But that this explanation is not sufficient, Babcock $†$ has shown thus :

Though it is true that water is a better conductor of heat than fat, the small size of the fat globules renders it impossible that, under any eircumstances, there can be more than a small fraction of a degree difference between the temperature of the fat and that of the milk serum. Moreover, within the limits of temperature practical for ereaming $\left(90^{\circ} \mathrm{F}\right.$. to $40^{\circ} \mathrm{F}$.), the

*American Dairying, p. 210.

† Wisconsin Agricultural Experiment Station, Bull. 18, p. 24. 
coefflcient of expansion of butter fat is more than three times as great as that of water, so that in order to maintain the same relative difference in their specific gravities, when the temperature is falling, the milk serum must cool more than three times as rapidly as the fat. In other words, when the milk serum has cooled from $90^{\circ} \mathrm{F}$. to $40^{\circ} \mathrm{F}$, or through $50^{\circ}$, the fat globules should have lost less than $17^{\circ}$, and should still have a temperature of over $73^{\circ} \mathrm{F}$, a difference between the temperature of the fat and serum of more than $33^{\circ}$. Such a condition is manifestly impossible, but any less difference than this would cause the fat to become relatively heavier than at first, and would operate against the creaming.

On the whole, the most satisfactory explanation of the good effect of the low temperature in the deep setting system is that advanced by Babcock, * that the presence of fibrin in milk, especially when it has coagulated in the form of threadlike masses, permeating the fluid in all directions, offers a considerable obstacle to the rising of the fat globules. The sudder reduction of the temperature quickly after the milk is drawn, by prevcnting the formation of these fibrin clots or threads, aids in the separation of the fat. This would be entirely satisfactory wore it not for the fact that it has been shown that while it is usually of advantage to cool the milk immediately after it is drawn, in some cases, at least, the setting and cooling may have been delayed for a time long enough to permit the formation of fibrin clots without appreciable effects upon the separation, as the following tables $\dagger$ very clearly show, the efficiency of creaming being measured by the percentage of fat in the skimmed milk:

* Loc. cit.

† Cornell University Agricultural Experiment Station, Bull. No. 29, p. 73. 
Effect of delayed setting upon creaming.

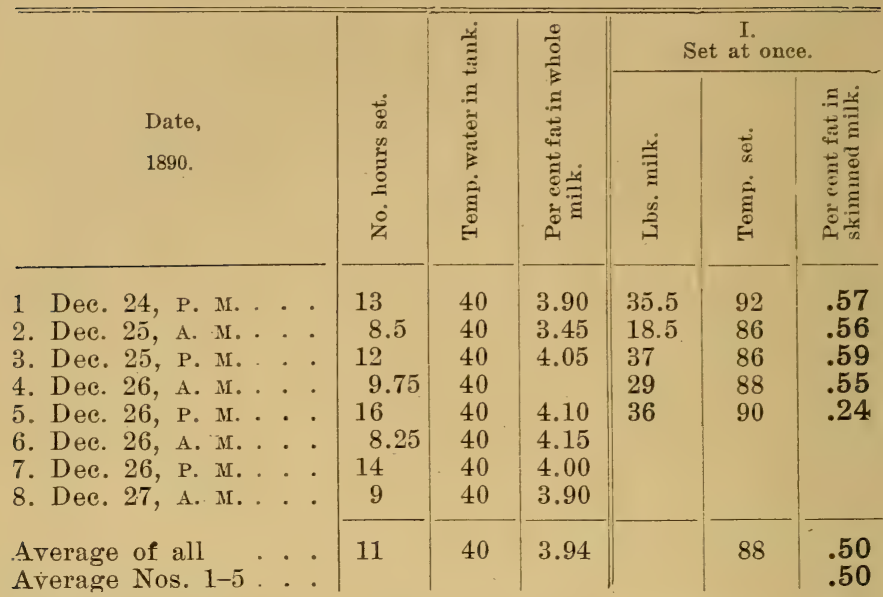

\begin{tabular}{|c|c|c|c|c|c|c|c|c|}
\hline \multirow[b]{2}{*}{$\begin{array}{l}\text { Date, } \\
1890 .\end{array}$} & \multicolumn{4}{|c|}{$\begin{array}{c}\text { II. } \\
\text { Delayed-kept warm. }\end{array}$} & \multicolumn{4}{|c|}{$\begin{array}{l}\text { III. } \\
\text { Delayed-cooled. }\end{array}$} \\
\hline & 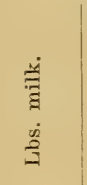 & 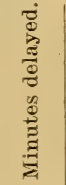 & 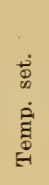 & 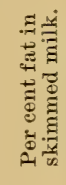 & 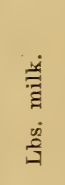 & 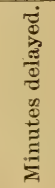 & 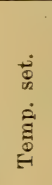 & 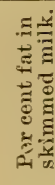 \\
\hline 1. Dec. 24, P. ห. & 24.5 & 60 & 88 & .59 & 30 & 60 & 68 & .64 \\
\hline 2. Dec. 25 , А. M. & 18.5 & 45 & 84 & .5 & 75.5 & 220 & 62 & .5 \\
\hline 3. Dec. 25 , Р. м. & & 45 & 84 & .4 & 61.5 & 60 & 74 & .53 \\
\hline 4. Dec. 26, A. M. & 30.25 & 130 & 82 & .68 & & & & \\
\hline 5. Dec. 26, Р. м. & 36 & 75 & 90 & .22 & & & & \\
\hline 6. Dec. 26, A. м. & 37.5 & 205 & 92 & .68 & 37.5 & 205 & 58 & .66 \\
\hline 7. Dec. 26, Р. м. & 24 & 90 & 90 & .4 & 23.5 & 90 & 65 & .6 \\
\hline 8. Dec. 27, A. M. & & 210 & 95 & & 74 & 210 & 64 & .51 \\
\hline $\begin{array}{l}\text { Average of all } \\
\text { Average Nos. } 1-5 \\
\text { Average } 6 \text { 6-8 }\end{array}$ & & 107 & 88 & $\begin{array}{l}.55 \\
.50 \\
.58\end{array}$ & & 141 & 65 & $\begin{array}{l}.59 \\
.57 \\
.60\end{array}$ \\
\hline
\end{tabular}


The milk was divided into three portions; one was set at once, one was delayed for an hour, but kept up to a temperature of 92 in a water bath, and the third was put in the open air and allowed to cool for an hour before set. In the cases of delay, the milk was stirred up just before it was placed in the creamer, and all the settings were made in Cooley cans, in ice water.

The trials reported under the same number, with the exception of the first and second, are comparable, as they were made from different portions of the same samples of milk. In numbers 1 to 5 , inclusive, the delayed sample (Column II.) was kept warm in a water bath; in numbers 6 to 8 the milk used had been carried about on the route, and the delayed sample was heated up after having been allowed to cool.

From a similar series of experiments Jordan* concluded:

That with herds of ordinary size, it will not be profitable to submit to any great inconvenience in order to place the milk in ice water immediately after it is drawn. In a half hour to an hour, milk does not seem to cool sufficiently to materially affect the completeness with which cream will rise.

In order to overcome certain difficulties that often occur in raising cream by deep setting process, dilution of the milk with water has been recommended, usually under one of the three following conditions:

1st. Dilution with one-fourth to one-third of hot water, $135^{\circ} \mathrm{F}$, and setting in deep cans at a temperature as low as can be obtained without the use of ice, not below $55^{\circ}$.

$2 d$. Dilution with one-half to equal quantities of

* Maine Agr. Exp. Sta. Rept. 1890, Part II. page 48. 
cold water, and setting under the same conditions as above.

Both of these being intended as substitutes for the use of ice in cool deep setting in the summer time.

3d. Dilution with one-third to one-fourth of hot water $\left(135^{\circ}\right)$, setting in deep eans in ice water $(40 \mathrm{~F}$.$) .$

This last intended to overcome the difficulty of complete creaming often found in the fall and early winter with the milk of cows far advanced in the period of lactation.

The idea is that the increased fluidity imparted to the milk by the water would facilitate the separation of the fat globules. During the winters of 1888-9 and 1889-90 very little ice could be harvested through the dairy regions of the northeastern United States, and in the summer following this idea of dilution was widely advocated and considerably practiced; but experience and experiment* have shown that while dilution may be of some advantage when, for lack of a supply of ice or other reasons, it is not possible to secure a temperature below $60^{\circ} \mathrm{F}$., it can in no case be considered a satisfactory substitute for setting the cans in water at a temperature of $40^{\circ} \mathrm{F}$.

The amount of advantage which may be obtained where dilution is practiced, as measured by the percentage of fat in the skimmed milk, is seen in the following tables (on pages 175 and 176), taken from Bulletin No. 39 of the Cornell University Agricultural Experiment Station:

*Vermont Agricultural Experiment Station, 4th Ann. Rept. p. 100. 
Diluted and undiluted milk set in water at a temperature of $60^{\circ} \mathrm{F}$.

\begin{tabular}{|c|c|c|c|c|c|c|c|c|c|c|c|c|c|c|}
\hline \multirow{2}{*}{\multicolumn{2}{|c|}{$\begin{array}{l}\text { Date, } \\
1892 .\end{array}$}} & \multirow[b]{2}{*}{ 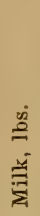 } & \multirow[b]{2}{*}{ 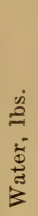 } & \multicolumn{4}{|c|}{ Temperatures $\mathrm{F}$. } & \multirow[b]{2}{*}{ 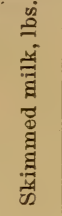 } & \multirow[b]{2}{*}{ 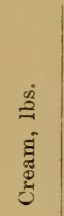 } & \multirow[b]{2}{*}{ 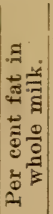 } & \multicolumn{2}{|c|}{ Diluted. } & \multicolumn{2}{|c|}{ Not diluted. } \\
\hline & & & & 光 & $\frac{\stackrel{\Phi}{\Xi}}{\vec{b}}$ & 苛 & 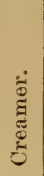 & & & & 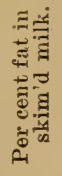 & 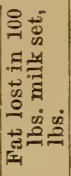 & 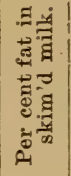 & 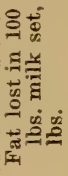 \\
\hline eb. & $\begin{array}{l}19 \\
19\end{array}$ & $\begin{array}{l}54 \\
72\end{array}$ & 18 & $\begin{array}{l}84 \\
84\end{array}$ & 135 & $\begin{array}{l}97 \\
84\end{array}$ & $\begin{array}{l}65 \\
65\end{array}$ & $\begin{array}{l}60 \\
59\end{array}$ & 12 & $\begin{array}{l}4.2 \\
4.2\end{array}$ & 1.07 & .89 & 1.33 & 1.09 \\
\hline "، & $\begin{array}{l}22 \\
22\end{array}$ & $\begin{array}{l}54 \\
72\end{array}$ & 18 & $\begin{array}{l}90 \\
90\end{array}$ & 136 & $\begin{array}{l}99 \\
90\end{array}$ & $\begin{array}{l}64 \\
64\end{array}$ & $\begin{array}{l}59.5 \\
57.5\end{array}$ & $\begin{array}{l}12.5 \\
14.5\end{array}$ & $\begin{array}{l}4.2 \\
4.2\end{array}$ & .52 & .43 & .97 & .77 \\
\hline ". & $\begin{array}{l}23 \\
23\end{array}$. & $\begin{array}{l}54 \\
72\end{array}$ & 18 & $\begin{array}{l}89 \\
89\end{array}$ & 135 & $\begin{array}{r}101 \\
89\end{array}$ & $\begin{array}{l}66 \\
66\end{array}$ & $\begin{array}{l}60.75 \\
58.50\end{array}$ & $\begin{array}{l}11.25 \\
13.5\end{array}$ & $\begin{array}{l}4.3 \\
4.3\end{array}$ & .80 & .68 & 1.12 & .91 \\
\hline "' & $\begin{array}{l}28 \\
28\end{array}$ & $\begin{array}{l}54 \\
72\end{array}$ & 18 & $\begin{array}{l}88 \\
88\end{array}$ & 134 & $\begin{array}{l}98 \\
88\end{array}$ & $\begin{array}{l}57 \\
57\end{array}$ & 58 & $\begin{array}{l}14 \\
15\end{array}$ & & .35 & .28 & .65 & .51 \\
\hline ". & $\begin{array}{l}29 \\
29\end{array}$. & $\begin{array}{l}54 \\
72\end{array}$ & 18 & $\begin{array}{l}86 \\
86\end{array}$ & 134 & $\begin{array}{l}97 \\
86\end{array}$ & $\begin{array}{l}60 \\
60\end{array}$ & $\begin{array}{l}59.75 \\
58\end{array}$ & $\begin{array}{l}12.25 \\
14\end{array}$ & $\begin{array}{l}4.4 \\
4.4\end{array}$ & .68 & .56 & 1.18 & .95 \\
\hline Mar. & $\begin{array}{l}1 . \\
1\end{array}$. & $\begin{array}{l}54 \\
72\end{array}$ & 18 & $\begin{array}{l}86 \\
86\end{array}$ & 135 & $\begin{array}{l}96 \\
86\end{array}$ & $\begin{array}{l}60 \\
60\end{array}$ & $\begin{array}{l}61 \\
58.5\end{array}$ & $\begin{array}{l}11 \\
13.5\end{array}$ & $\begin{array}{l}4.1 \\
4.1\end{array}$ & .68 & .58 & .96 & .78 \\
\hline ". & $\begin{array}{l}13 \\
13\end{array}$ & $\begin{array}{l}54 \\
72\end{array}$ & 18 & $\begin{array}{l}86 \\
86\end{array}$ & 135 & $\begin{array}{l}96 \\
86\end{array}$ & $\begin{array}{l}60 \\
60\end{array}$ & $\begin{array}{l}60.75 \\
57.75\end{array}$ & $\begin{array}{l}11.25 \\
14.25\end{array}$ & $\begin{array}{l}4.3 \\
4.3\end{array}$ & .85 & .72 & .82 & .66 \\
\hline " & $\begin{array}{l}14 \\
14\end{array}$ & $\begin{array}{l}27 \\
36\end{array}$ & 9 & $\begin{array}{l}84 \\
84\end{array}$ & 136 & $\begin{array}{l}96 \\
84\end{array}$ & $\begin{array}{l}58 \\
58\end{array}$ & $\begin{array}{l}29.5 \\
30\end{array}$ & $\begin{array}{l}6.5 \\
6\end{array}$ & \begin{tabular}{|l|}
3.9 \\
3.9
\end{tabular} & .88 & .72 & 1.14 & .95 \\
\hline “. & $\begin{array}{l}15 \\
15\end{array}$ & $\begin{array}{l}2 \\
3\end{array}$ & 9 & $\begin{array}{l}8 \\
8\end{array}$ & 132 & $\begin{array}{l}95 \\
86\end{array}$ & $\begin{array}{l}58 \\
58\end{array}$ & $\begin{array}{l}30 \\
29.5\end{array}$ & $\begin{array}{l}6 \\
6.5\end{array}$ & \begin{tabular}{l|}
3.9 \\
3.9
\end{tabular} & .73 & .61 & 1.25 & 1.02 \\
\hline "1" & $\begin{array}{l}26 \\
27\end{array}$ & 27 & 9 & $\begin{array}{l}88 \\
88\end{array}$ & 136 & $\begin{array}{r}101 \\
88\end{array}$ & $\begin{array}{l}56 \\
56\end{array}$ & $\begin{array}{l}29.5 \\
28.5\end{array}$ & $\begin{array}{l}6.5 \\
7.5\end{array}$ & $\begin{array}{l}4.1 \\
4.1\end{array}$ & & & .67 & .53 \\
\hline ". & $\begin{array}{l}27 \\
27\end{array}$. & $\begin{array}{l}54 \\
36\end{array}$ & 18 & $\begin{array}{l}90 \\
90\end{array}$ & 135 & $\begin{array}{r}100 \\
90\end{array}$ & $\begin{array}{l}64 \\
64\end{array}$ & $\begin{array}{l}60.25 \\
30\end{array}$ & $\underset{6}{11.75}$ & $\begin{array}{l}4.5 \\
4.5\end{array}$ & .81 & .68 & .70 & .58 \\
\hline$"$ & $\begin{array}{l}28 \\
28\end{array}$ & $\begin{array}{l}27 \\
36\end{array}$ & 9 & $\begin{array}{l}88 \\
88\end{array}$ & 136 & $\begin{array}{r}102 \\
88\end{array}$ & $\begin{array}{l}61 \\
61\end{array}$ & $\begin{array}{l}31 \\
30\end{array}$ & $\begin{array}{l}5 \\
6\end{array}$ & 4 & & & 1.21 & 1.01 \\
\hline ". & $\begin{array}{l}31 \\
31\end{array}$. & $\begin{array}{l}27 \\
36\end{array}$ & 9 & $\begin{array}{l}87 \\
87\end{array}$ & 135 & $\begin{array}{r}102 \\
87\end{array}$ & $\begin{array}{l}62 \\
62\end{array}$ & $\begin{array}{l}30 \\
29.75\end{array}$ & $\begin{array}{l}6 \\
6.25\end{array}$ & $\begin{array}{l}4.1 \\
4.1\end{array}$ & .93 & .78 & 1.17 & .97 \\
\hline Apr. & $\begin{array}{l}2 \\
2\end{array}$ & $\begin{array}{l}27 \\
36\end{array}$ & 9 & $\begin{array}{l}91 \\
91\end{array}$ & 135 & $\begin{array}{r}103 \\
91\end{array}$ & $\begin{array}{l}65 \\
65\end{array}$ & $\begin{array}{l}30 \\
29\end{array}$ & $\begin{array}{l}6 \\
7\end{array}$ & $\begin{array}{l}4.7 \\
4.7\end{array}$ & 1.08 & .90 & 1.68 & 1.35 \\
\hline " & $\begin{array}{l}4 \\
4\end{array}$ & $\begin{array}{l}27 \\
36\end{array}$ & 9 & $\begin{array}{l}92 \\
92\end{array}$ & 135 & $\begin{array}{r}102 \\
92\end{array}$ & $\begin{array}{l}62 \\
62\end{array}$ & $\begin{array}{l}29 \\
29\end{array}$ & $\begin{array}{l}7 \\
7\end{array}$ & $\begin{array}{l}4.2 \\
4.2\end{array}$ & .31 & .25 & .92 & .74 \\
\hline & & . . & & . & $\ldots$ & - & . & & & & .76 & .62 & 1.05 & .85 \\
\hline
\end{tabular}


Diluted and undiluted milk set in water at a temperature of $40^{\circ}-45^{\circ} \mathrm{F}$.

\begin{tabular}{|c|c|c|c|c|c|c|c|c|c|c|c|c|c|}
\hline \multirow[b]{2}{*}{$\begin{array}{c}\text { Datel, } \\
1892 .\end{array}$} & \multirow[b]{2}{*}{$\begin{array}{l}\stackrel{\dot{D}}{Z} \\
\stackrel{\vec{Z}}{\Xi}\end{array}$} & \multirow[b]{2}{*}{ 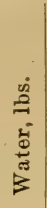 } & \multicolumn{4}{|c|}{ Temperatures F. } & \multirow[b]{2}{*}{ 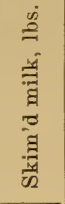 } & \multirow[b]{2}{*}{ 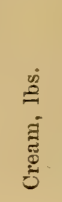 } & \multirow[b]{2}{*}{ 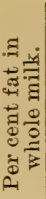 } & \multicolumn{2}{|c|}{ Diluted. } & \multicolumn{2}{|c|}{ Not diluted. } \\
\hline & & & 尝 & 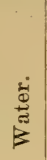 & $\begin{array}{c}\dot{0} \\
\ddot{D}\end{array}$ & 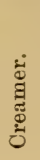 & & & & 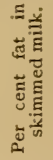 & 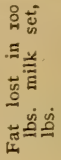 & 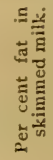 & 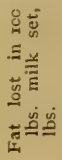 \\
\hline Feb. 20 . & $\begin{array}{l}54 \\
72\end{array}$ & 18 & $\begin{array}{l}87 \\
87\end{array}$ & 140 & $\begin{array}{r}100 \\
87\end{array}$ & $\begin{array}{l}44 \\
44\end{array}$ & $\begin{array}{l}58 \\
54.25\end{array}$ & $\begin{array}{l}14 \\
16.75\end{array}$ & $\begin{array}{l}4.4 \\
4.4\end{array}$ & .32 & .26 & .25 & .19 \\
\hline$\because \quad 21$. & $\begin{array}{l}54 \\
72\end{array}$ & 18 & $\begin{array}{l}88 \\
88\end{array}$ & 136 & $\begin{array}{l}99 \\
88\end{array}$ & $\begin{array}{l}44 \\
44\end{array}$ & $\begin{array}{l}57.5 \\
53.75\end{array}$ & $\begin{array}{l}14.5 \\
18.25\end{array}$ & $\begin{array}{l}4.4 \\
4.4\end{array}$ & .20 & .16 & .24 & .18 \\
\hline $\begin{array}{ll}\because & 24 \\
\because & 24 .\end{array}$ & $\begin{array}{l}54 \\
72\end{array}$ & 18 & $\begin{array}{l}87 \\
87\end{array}$ & 134 & $\begin{array}{l}97 \\
87\end{array}$ & $\begin{array}{l}45 \\
45\end{array}$ & $\begin{array}{l}56.25 \\
53.5\end{array}$ & $\begin{array}{l}15.75 \\
18.5\end{array}$ & $\begin{array}{l}4.2 \\
4.2\end{array}$ & .23 & .18 & .17 & .13 \\
\hline $\begin{array}{ll}\text { “ } 25 . \\
\text { “ } 25 .\end{array}$ & $\begin{array}{l}54 \\
72\end{array}$ & 18 & $\begin{array}{l}90 \\
90\end{array}$ & 134 & $\begin{array}{r}100 \\
90\end{array}$ & $\begin{array}{l}46 \\
46\end{array}$ & $\begin{array}{l}57 \\
54.25\end{array}$ & $\begin{array}{l}15 \\
17.75\end{array}$ & $\begin{array}{l}4.4 \\
4.4\end{array}$ & .23 & .18 & .26 & .20 \\
\hline $\operatorname{Mar}_{30} 30$ & $\begin{array}{l}27 \\
36\end{array}$ & 9 & $\begin{array}{l}86 \\
86\end{array}$ & 135 & $\begin{array}{r}100 \\
86\end{array}$ & $\begin{array}{l}44 \\
44\end{array}$ & $\begin{array}{l}29 \\
27\end{array}$ & $\begin{array}{l}7 \\
9\end{array}$ & & .32 & .26 & .35 & .26 \\
\hline$\because \quad 17$. & $\begin{array}{l}27 \\
18\end{array}$ & 9 & $\begin{array}{l}88 \\
88\end{array}$ & 136 & $\begin{array}{l}97 \\
88\end{array}$ & $\begin{array}{l}44 \\
44\end{array}$ & $\begin{array}{l}29 \\
13.5\end{array}$ & $\begin{array}{l}7 \\
4.5\end{array}$ & $\begin{array}{l}4.7 \\
4.7\end{array}$ & .11 & .09 & .18 & .14 \\
\hline $\begin{array}{ll}“ \quad 30 . \\
“ \quad 30 .\end{array}$ & $\begin{array}{l}27 \\
18\end{array}$ & 9 & $\begin{array}{l}86 \\
86\end{array}$ & 135 & $\begin{array}{r}100 \\
86\end{array}$ & $\begin{array}{l}44 \\
44\end{array}$ & $\begin{array}{l}28 \\
13\end{array}$ & $\begin{array}{l}8 \\
5\end{array}$ & $\begin{array}{l}5 \\
5\end{array}$ & .08 & .06 & .12 & .09 \\
\hline April 5 & $\begin{array}{l}27 \\
18\end{array}$ & 9 & $\begin{array}{l}90 \\
90\end{array}$ & 138 & $\begin{array}{r}103 \\
90\end{array}$ & $\begin{array}{l}46 \\
46\end{array}$ & $\begin{array}{l}29.75 \\
14.5\end{array}$ & $\begin{array}{l}6.25 \\
3.5\end{array}$ & $\begin{array}{l}3.3 \\
3.3\end{array}$ & .31 & .25 & .28 & .23 \\
\hline Average & . & . & . & & . & & 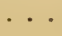 & . & .. & .23 & .18 & .23 & .18 \\
\hline
\end{tabular}

Summing up all of the experiments, the average efficiency of creaming as measured by the percentage of fat in the skimmed milk would appear to be about as follows :

Diluted, set at $60^{\circ}$ (39 trials) .77 per cent.

Undiluted, set at $60^{\circ}(30$ "“ $) 1.00$

$$
\text { “" " } 40^{\circ}(26 \text { ، }) \quad .29
$$

It would seem, therefore, that while, when the milk is set at $60^{\circ} \mathrm{F}$. or thereabouts, there is consider able advantage, so far as the efficiency of creaming 
is concerned, in diluting it with 25 per cent of warm water, this dilution cannot be regarded as a substitute for setting without dilution in ice water, and it has the further disadvantage of requiring increased tank capacity.

About 1897 the idea that dilution with water is an important aid in gravity-creaming broke out with renewed activity. It was especially recommended by the manufacturer's of certain forms of cheap tin cans in which dilution was recommended as an essential part of the process. These cans were called gravity "separators" modified by various high-sounding, qualifying phrases, with the evident intention of conveying the idea that this process was as efficient as centrifugal separation, and large numbers of the "separators" have been sold, mainly to unsuspecting or ignorant farmers, who have been deluded into the idea that they were securing a contrivance equal in efficiency to a centrifugal separator at a small fraction of the cost. The form of many of the cans was patented, but it was s on show * that so far as the process is concerned, the patents were valueless, and trials at several experiment stations showed that dilution in gravity separators, of whatever form, is no more efficacious than has been shown above. For this reason, and because rival manufacturers have become involved in controversies over their various patents, the "dilution separator boom" is, fortunately, likely to be of short duration.

Centrifugal separation. - The invention, development and perfection of the centrifugal separator has

*Cornell University Agricultural Experiment Station, Bulls. 151 and 171. 
been the chief factor in revolutionizing methods of butter-making. By its greater efficiency it has prevented otherwise unavoidable losses, and by its greater economy of labor it has rendered possible the development of a profitable industry in many localities where it would have been otherwise impossible.

In separating cream in a centrifugal machine, the centrifugal force generated in a rapidly revolving bowl is made to take the place of the force of gravity acting upon the milk at rest in a vessel. The amount of force generated is so much greater than the force of gravity that the separation of the particles of fat is much more rapid and much more complete. The force, however, acts in a horizontal instead of a vertical direction. In 1877 , a patent was granted to Le Feldt \& Lentsch for a machine to separate milk by centrifugal force. This first centrifugal separator consisted merely of a series of buckets hung upon arms swinging from a central axis. When the machine was at rest the buckets assumed a vertical position, but in motion they were thrown out horizontally from the arms. The milk was placed in these buckets, the machine set in motion until the cream was separated from the skimmed milk, and when the machine was allowed to come to a stand-still the buckets assumed a vertical position, and the cream was removed from the top in the same way that it was skimmed from any other vessel. From this was evolved a machine consisting of a revolving bowl or drum in which the separation takes place, with arrangements for removing the skimmed milk and 
cream without stopping the machine, thus making the separation continuous.

This constituted the first practical cream separator. It was the invention of Dr. Gustaf de Laval, a Swedish inventor.

The various parts of the machine have since been much improved in minor details: At the present time the essential parts of a separator are the bowl, with or without internal devices or arrangements to assist in the separation of the cream from the milk, an

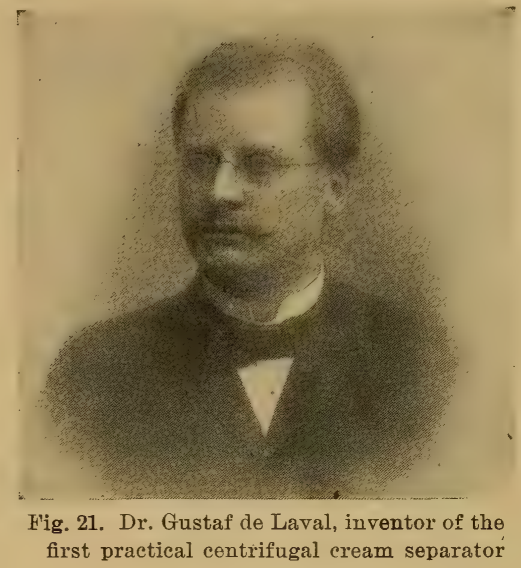

outlet for the skimmed milk, an outlet for the cream, an inflow for the whole milk, and the proper mechanical means for revolving the bowl. (The more common types of separator bowls are shown in Figs. 10-13.) Usually the bowl is driven in an upright position, but there are separators in which the bowl is driven in a horizontal position, and in the greater number of machines the walls of the bowl are cylindrical. In the process of separation the milk flows into the bowl, and, partaking of the centrifugal force, is forced to the extreme outer edge of the bowl. As the milk continues to flow in, the bowl begins 
to fill from the outside toward the center. The centrifugal force acting more strongly upon those parts of the milk which have the greatest specific gravity, they are thrown to the extreme outside, and the lighter portions, the fat globules, with whatever may adhere to them, are forced to the center. Attached to the extreme outer edge of the bowl are one or more tubes, which, bending inward along the side of the bowl, find an opening near the center. These are the outlets for the skimmed milk. From the extreme center of the bowl, also leading to the outside, is the cream ontlet. When the bowl becomes filled with milk, the centrifugal $L$ pressure will force out through the skimmed milk outlets the milk

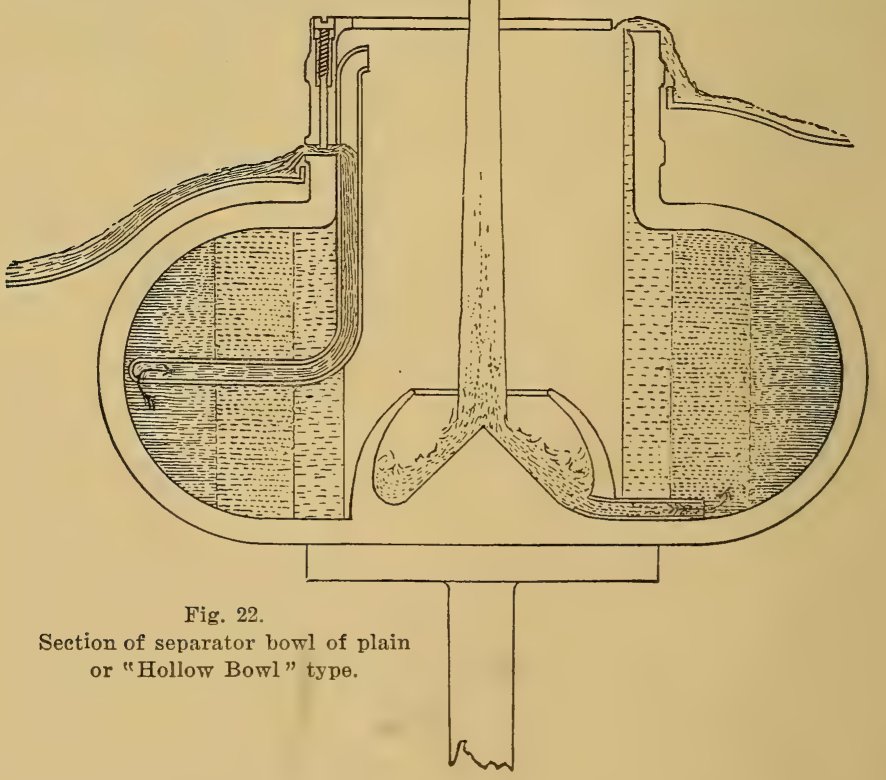


nearest the outside of the bowl. These outlets are made of such size in comparison with the size of the bowl, the speed of the machine, and the size of the inflow tube, that they have a capacity of discharging the milk from .4 to .9 as fast as it runs in; the remaining contents of the bowl are then forced toward the center, and find an exit through the cream outlet. In this way the milk as it enters is divided into two portions: one, the larger, drawn from the extreme outer portion of the bowl, consisting of skimmed milk, and the other, the smaller, escaping from the center of the bowl, the cream. A third portion also usually accompanies centrifugal separation. A part of the semi-solid constituents of the milk, being heavier than the milk serum, are thrown to the outside of the bowl, and adhere to the walls in the form of a creamy or gelatinous mass which is ordinarily called separator slime. It consists mainly of albuminous matter. with some fat globules adhering, and any particles of dirt or foreign matter that may be mechanically mixed with the milk. It varies greatly at different times and seasons. Usually the amount is not large, but not infrequently it is sufficient to clog the outlets of the separator after a run of an hour or two. The separator slime is largely composed of matter deleterious to the quality of cream and butter, and its removal is to a great extent a purification of the cream. The various conditions affecting this operation are as follows:

Conditions affecting the completeness of separation.The completeness of the separation is dependent upon 
the centrifugal force generated, the rate of inflow, the temperature of the milk, and the physical condition of the milk.

The amount of centrifugal force generated depends upon the diameter of the bowl and the velocity of rotation.

Roughly speaking, the centrifugal force increases directly in proportion as the diameter is increased and directly with the square of the velocity. The larger the bowl, then, and the greater the speed, the greater the centrifugal force and the more complete the separation. The rate of inflow of milk affects the separation, because the separation is more complete the longer the milk is subjected to the centrifugal force, and the slower the milk flows into a givensized bowl the longer any particle will be in flowing through it and the more completely will the cream be separated. The temperature of the milk affects 'the fluidity of the fat globules and their ease of motion upon the other particles of the milk; the warmer the milk the more easily are they separated. A temperature of $76^{\circ}$ to $98^{\circ} \mathrm{F}$. is the one commonly employed because of the effect upon the texture of the butter. It is desirable that the milk should be separated at as low a temperature as possible without interfering with the completeness of the separation, so that, other things being equal, that separator is the best that separates the milk at the lowest temperature. The physical condition of the milk affects separation by the centrifugal in the same ways that gravity creaming is affected, but to a very much 
slighter degree. Small-sized fat globules, viscosity of the milk, and coagulation of part of the casein by incipient fermentations, all tend to make separation more difficult; but in a majority of cases, unless the milk is so sour as to be coagulated, it may be completely creamed with a centrifugal separator, but will require a slower feeding (a reduction of 10 to 15 per cent will usually suffice), and a higher speed of the bowl, or both.

Conditions affecting the relative amount of shimmed milk and cream.-The relative amount of skimmed milk and cream is affected by the rate of inflow, by the speed of the bowl, by various special contrivances upon the machines themselves, and to a slight extent by the temperature of the milk. In the ordinary machine the size of the skimmed milk outlet is fixed, and therefore at a given velocity the outlet will discharge a nearly uniform quantity of fluid. If then, the milk is turned into the bowl at such a rate that .8 of it escapes through the skimmed milk outlet, we shall have .8 skimmed milk and .2 cream. If now we reduce the rate of inflow by .1, we shall get just as much skimmed milk as before, but only one-half as much cream; or if the inflow is increased by .1, we shall get the same amount of skimmed milk and once and a half as much cream. If under the first conditions all of the fat was gathered into the cream, we shall have just as complete a separation as before, but shall simply get a cream containing a greater or less percentage of fat, provided that the increase of inflow has not increased the total amount above the capacity of the 
machine. In other words, the greater the inflow, the more cream and the thinner the cream; the smaller the inflow, the less cream and the richer the cream. These varations can only take place within comparatively narrow limits. If we increase the inflow too much, we shall soon reach a point at which the milk passes so rapidly through the machine that the separation is not complete, and if we reduce the inflow to such a point that the skimmed milk outlet has a capacity of discharging the milk as fast as it flows in, we shall get a separation until the bowl becomes filled, and then all of the milk will pass out of the skimmed milk outlet in the same condi tion in which it enters the machine.

The speed of the machine, also, affects the relative amount of cream and its percentage of fat. The size of the skimmed milk outlet being fixed, the faster the bowl is revolved the greater the capacity of this outlet will be, so that, the rate of inflow remaining uniform, the faster the bowl is revolved the less proportional amount of cream we shall have, and the richer it will be in fat, and vice versa. It must be borne in mind, further, that the speed of the bowl is also an important factor in the completeness of separation, and that if the speed is slackened in order to get a greater bulk of cream, there will be danger of incomplete separation.

Most of the machines have arrangements for regulating the relative amount of skimmed milk and cream without changing the rate of inflow or the speed of the machine. In most of the machines 
this arrangement is known as a "cream screw," and affects the amount of cream by placing the cream outlet nearer or farther from the center of the bowl; the nearer the center the cream screw is turned, the thicker will be the cream and the smaller the amount. Some of the separators change the proportion of cream by increasing or decreasing the capacity of the skimmed milk outlet. If the capacity of the skimmed milk outlet be decreased, necessarily the amount of the cream will be increased, and vice versa.

The temperature of the milk only slightly affects the proportion of skimmed milk and cream. The warmer the milk, the more fluid it is, and the faster it will pass through the various openings of the machine. Slightly more milk at $85^{\circ}$ will run through the same inflow than at $70^{\circ}$, and a large part of this increase will be found in the cream. The pressure of the milk in the vat above the machine will materially affect the rate of inflow, and a few inches will make a difference of two or three hundred pounds per hour on a large-sized machine.

Contrivances in the bowl to increase the efficiency of separation. - In the iirst separators made, the bowl
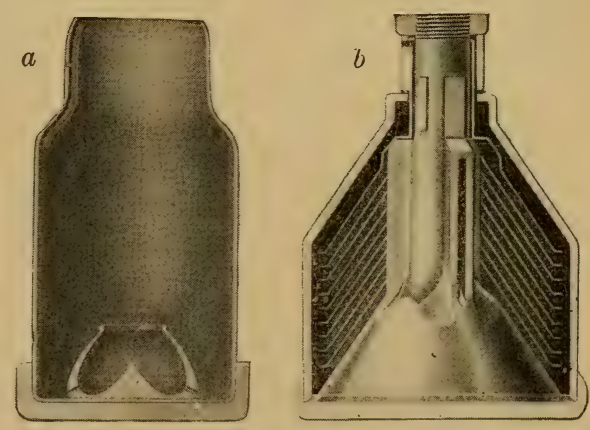

Fig. 23. a, Sectional view of old style "Hollow" separator bowl; $b$, Sectional view of "Alpha-Dise" separator bowl. 


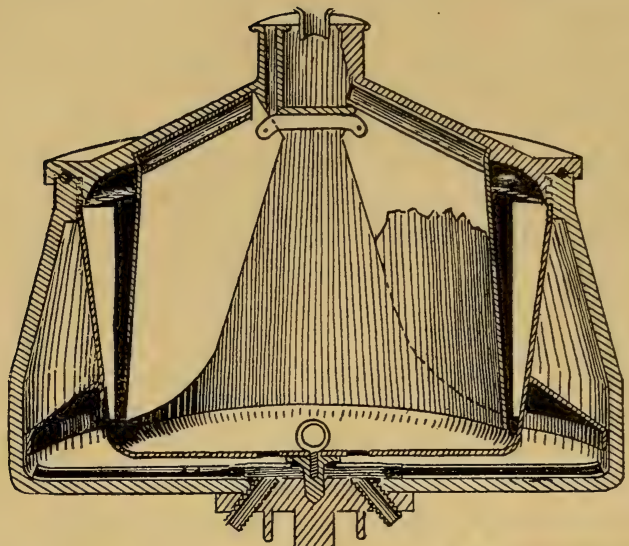

was hollow, and the separation was caused directly by the centrifugal pressure acting upon the milk in a mass. In the later machines, various contrivances have been introduced in the bowl to

Fig. 24. Section of separator bowl of "travel" type.

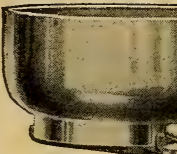

aid in the completeness of the separation or to increase the capacity. These contrivances have been of two general types: one to break up the wall of milk, and so give the particles of milk and cream a better chance to pass by one another in their passage from the center to the outside of the bowl; the other, a series of interruptions to

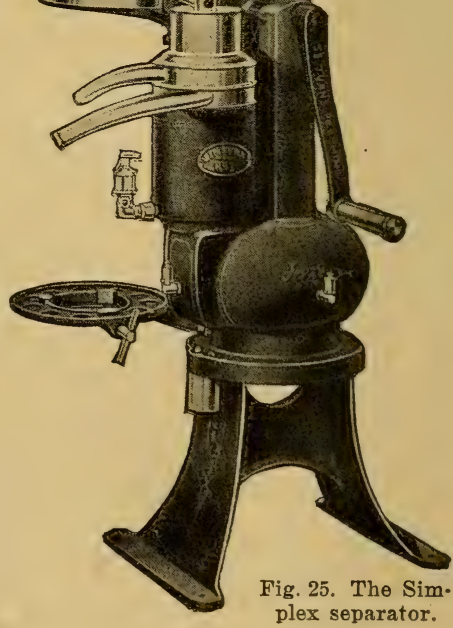


the passage of the milk from its entrance at the center to the outside, causing it to travel a much greater distance and be subjected to the centrifugal force for a longer time. These contrivances, while adding to the complexity of the machine, have increased the capacity and the efficiency of the separation. The best known of these contrivances are the so-called "Alpha" dises or
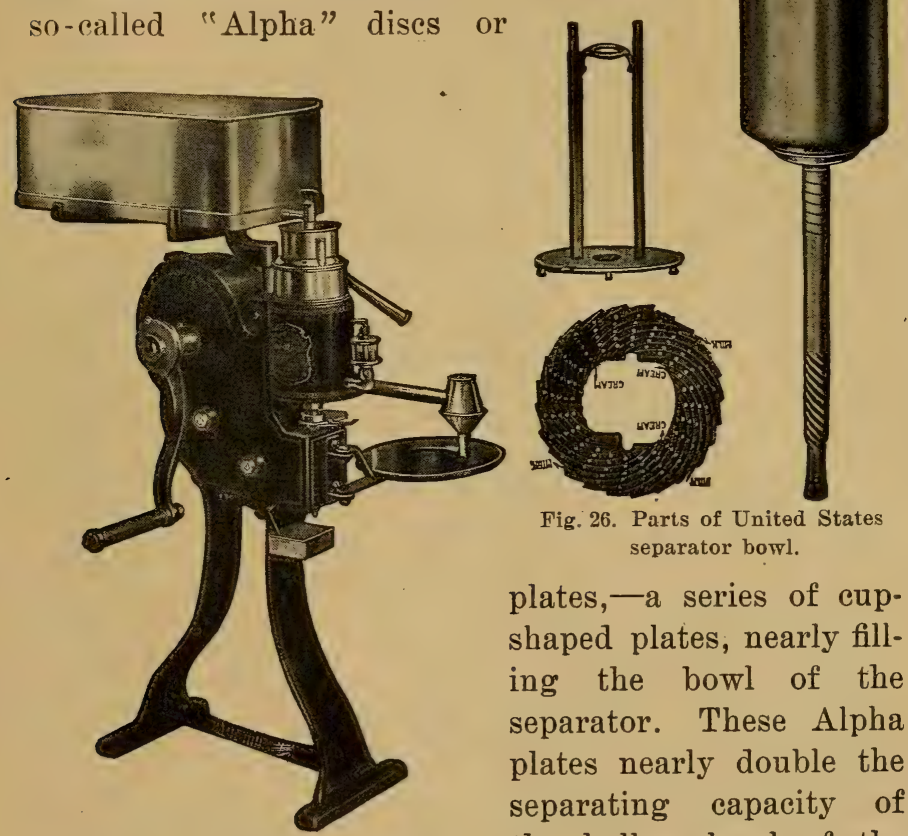

Fig. 26. Parts of United States separator bowl.

plates, - a series of cupshaped plates, nearly filling the bowl of the separator. These Alpha plates nearly double the separating capacity of Fig. 27. United States separator. the hollow bowl of the 
same diameter, and at the same time the efficiency of separation is increased.

In the latest developments in separator construction various other devices intended to secure the same end

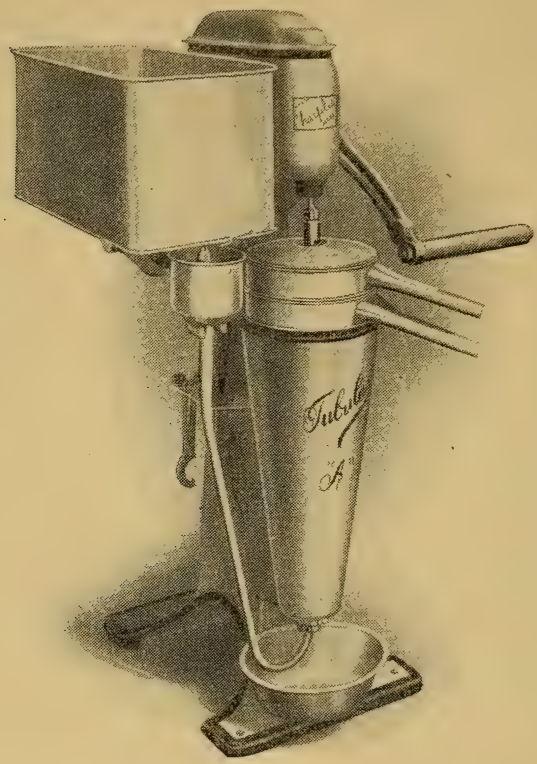

Fig. 28. 'Tubular separator

have been more or less successfully used. Among the more recent of these the simplex (Fig. 25) and the tubular (Fig. 28) should be mentioned. The latter has been particularly successful in securing efficiency by making the bowl very long and of very small diameter and running it at a comparatively high speed. 
Mechanical conditions which affect separation.-Certain peculiarities in the construction or operation of the machine often materially influence the efficiency of separation. One of the most important of these is that the bowl should run steadily and smoothly, without any preceptible jar or trembling. The trembling of the bowl may be caused by lack of perfect balancing in the bowl itself. This can be remedied only by the manufacturer. The bowl may tremble, also, because the bearings are not perfectly adjusted; and, lastly, the bowl may tremble because the machine is not set level or upon a solid foundation. Often a bowl will tremble somewhat while it is speeding up but will run smoothly when full speed is attained; such a bowl, however, will seldom work as perfectly as one that is so balanced that it will run at any and all speeds without perceptible jar. A second cause of inefficient separaation is variation in the

Fig. 29.

Parts of tubular bowl. velocity of the bowl. It is quite as important that the

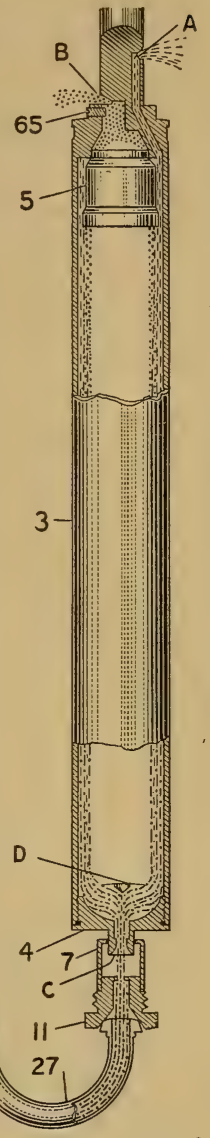

Fig. 30, Section of tubular separator. A. skim milk vent; $B$, cream vent; $C$, milk inlet; $D_{1}$ wing : 3 , bowl ; 4 , bowl bottom; 5 , dividuing wall; 7. drag shell; 11, nozzle; 27 , feed tube; 65 , cream screw. 
bowl run uniformly as it is that it attain any given rate of velocity. In this respect the turbine separators are more likely to be at fault than those run by belt power, and separators turned by hand are more subject to variations than those run by power.

An engine of ample power, with a good governor, and the power transmitted through an intermediate rope belt kept perfectly tightened, with well-oiled bearings all around, are the best safeguards to uniform speed.

Efficiency of separation in centrifugal machines.With the centrifugal separator run under perfect conditions, there is still a slight loss of fat in the skimmed milk. This should not be greater than .1 of 1 per cent. At the present time it is considered that where as much as .1 of 1 per cent of fat is left in the skimmed milk a centrifugal machine is doing such poor work that its use in a commercial plant would be unwarranted. The following tables* taken from the average of a large number of tests made by several agricultural experiment stations may be taken as representing the degree of efficiency that had been attained by the leading manufacturers at the time when separators first came into general use (1890-95) and the first table is compiled wholly from American sources. At the present time no separator should be kept in use that will leave more than two or three hundredths of one per cent of fat in the skimmed milk in a series of tests running day after day under ordinary factory conditions.

\footnotetext{
*Cornell University Agricultural Experiment Station, Bulletin No. 105.
} 


\begin{tabular}{|c|c|c|c|c|c|}
\hline \multirow{3}{*}{\multicolumn{2}{|c|}{ Name of machine. }} & \multicolumn{4}{|c|}{ Per cent of fat in skimmed milk. } \\
\hline & & \multicolumn{2}{|c|}{ Average. } & \multirow[b]{2}{*}{ Minimum. } & \multirow[b]{2}{*}{ Maximum. } \\
\hline & & By series. & $\begin{array}{l}\text { Of all } \\
\text { trials. }\end{array}$ & & \\
\hline Accumulator & . . & .12 & .11 & .01 & .20 \\
\hline Alexandra Jumbo & . . & .22 & .22 & .15 & .33 \\
\hline Columbia ... & . . & .09 & .12 & .05 & .34 \\
\hline Danish Weston . & . . & .10 & .08 & .01 & .25 \\
\hline DeLaval ..... & . . & .13 & .09 & .01 & .50 \\
\hline Sharples . . . . & & .27 & .16 & .05 & .65 \\
\hline United States.. & . . & .18 & .12 & .01 & .60 \\
\hline Victoria .. . . . & . & .21 & .16 & .05 & .38 \\
\hline
\end{tabular}

A series of trials made in Germany* shows a considerable variation in the various styles, and, on the whole, a rather less efficiency than has been obtained in this country, as is seen in the table following:

\begin{tabular}{|c|c|c|c|c|c|}
\hline Name of machine. & $\begin{array}{c}\text { Revolutions } \\
\text { of bowl } \\
\text { per min- } \\
\text { ute. }\end{array}$ & $\begin{array}{l}\text { Pounds } \\
\text { separated } \\
\text { per hour. }\end{array}$ & $\begin{array}{l}\text { Tempera- } \\
\text { ture of } \\
\text { milk F. }\end{array}$ & $\begin{array}{l}\text { Per cent } \\
\text { of fat in } \\
\text { skimmed } \\
\text { milk. }\end{array}$ & $\begin{array}{l}\text { Number } \\
\text { of trials. }\end{array}$ \\
\hline $\begin{array}{l}\text { Alpha B } \\
\text { Alpha Baby . } \\
\text { Baby : } \\
\text { Victoria : } \\
\text { Small Danish : } \\
\text { Large Danish . } \\
\text { Adjustable.. } \\
\text { Arnold's . } \\
\text { Medium Brown }\end{array}$ & $\begin{array}{l}5,100 \\
6,900 \\
6,360 \\
\dot{7}, 241 \\
7,217 \\
8,640 \\
8,531 \\
4,037\end{array}$ & $\begin{array}{l}537 \\
286 \\
129 \\
205 \\
288 \\
411 \\
363 \\
458 \\
268\end{array}$ & $\begin{array}{l}92 \\
72 \\
88 \\
92 \\
90 \\
91 \\
70 \\
90 \\
91\end{array}$ & $\begin{array}{l}.23 \\
.23 \\
.18 \\
.17 \\
.28 \\
.35 \\
.10 \\
.28 \\
.37\end{array}$ & $\begin{array}{r}37 \\
23 \\
15 \\
19 \\
16 \\
16 \\
3 \\
26 \\
16\end{array}$ \\
\hline
\end{tabular}

But while all the forms of separators can be made to do perfect work, there is more or less variation

* Mileh Zeitung, xxiii., p. 296. 
in the work done by the different machines of the same style and manufacture. This variation is due to slight peculiarities in the construction of the individual bowls, and which cannot be detected by the ordinary senses, and only appear upon an examination of the skimmed milk after actual use. These differences are well illustrated in a series of trials made in different factories, ${ }^{*}$ and shown in detail in the following tables:

\section{ALEXANDRA JUMBO}

Rated capacity, 2,000 pounds per hour

\begin{tabular}{|c|c|c|c|c|c|c|c|c|}
\hline Date. & $\begin{array}{c}\text { No. } \\
\text { of } \\
\text { fac- } \\
\text { tory. }\end{array}$ & $\begin{array}{c}\text { Pounds } \\
\text { of } \\
\text { milk. } \\
\text { in whole } \\
\text { run. }\end{array}$ & $\begin{array}{c}\text { Average } \\
\text { temper- } \\
\text { ature. }\end{array}$ & $\begin{array}{c}\text { Range } \\
\text { of } \\
\text { temper- } \\
\text { ature. }\end{array}$ & $\begin{array}{c}\text { Average } \\
\text { speed of } \\
\text { bowl, } \\
\text { revolu- } \\
\text { tions per } \\
\text { minute. }\end{array}$ & $\begin{array}{c}\text { Range } \\
\text { of } \\
\text { speed. }\end{array}$ & $\begin{array}{c}\text { Pounds } \\
\text { sepa- } \\
\text { rated } \\
\text { per } \\
\text { hour. }\end{array}$ & $\begin{array}{c}\text { Per cent } \\
\text { of fat in } \\
\text { skimmed } \\
\text { milk. }\end{array}$ \\
\hline $\begin{array}{cc}\text { Aug. } & 19 . \\
\text { “ } & 20 . \\
\text { “ } & 20 . \\
\text { “ } & 23 .\end{array}$ & $\begin{array}{r}7 \\
8 \\
8 \\
9 \\
11\end{array}$ & 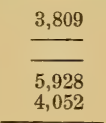 & $\begin{array}{l}70 \\
70 \\
70 \\
75 \\
84\end{array}$ & $\begin{array}{l}66-75 \\
68-72 \\
68-72 \\
72-81 \\
80-86\end{array}$ & $\begin{array}{l}7,200 \\
6,985 \\
6,585 \\
6,900 \\
6,600\end{array}$ & $\begin{array}{l}7,000-7,400 \\
6,800-7,200 \\
6,300-6,900 \\
6,600-7,100 \\
6,200-7,400\end{array}$ & $\begin{array}{l}1,344 \\
1,170 \\
1,611 \\
1,882 \\
1,814\end{array}$ & $\begin{array}{l}.25 \\
.15 \\
.20 \\
.20 \\
.33\end{array}$ \\
\hline Average & $\cdots$ & $\cdots$ & 74 & . & 6,854 & $\cdots$ & 1,564 & .23 \\
\hline
\end{tabular}

DE LAVAL, ALPHA NO. 1

Rated capacity, 2,500 pounds per hour

\begin{tabular}{|c|c|c|c|c|c|c|c|c|}
\hline July 17 . & 3 & 2,519 & 80 & $78-85$ & 5,520 & $4,800-6,100$ & 2,606 & .08 \\
\hline Aug. 18. & 6 & 3,629 & 74 & $73-76$ & 5,806 & $5,700-6,000$ & 2,592 & .05 \\
\hline Sep. 16. & 17 & 1,187 & 72 & $71-73$ & 5,933 & $5,800-6,000$ & 2,456 & .08 \\
\hline 20 . & 22 & 4,627 & 82 & $80-83$ & 6,071 & $5,800-6,200$ & 2,501 & .04 \\
\hline " 21 . & 23 & 6,376 & 78 & $77-80$ & 6,044 & $6,000-6,200$ & 2,500 & .03 \\
\hline Oct. 4. & 24 & 5,588 & .82 & $78-85$ & 5,844 & $5,600-6,000$ & 2,747 & .13 \\
\hline & 25 & 1,802 & 86 & $85-90$ & 6,280 & $6,000-6,400$ & 2,040 & .03 \\
\hline Average & - & - & 79 & . . & 5,928 & . . . & 2,491 & .06 . \\
\hline
\end{tabular}

*Cornell University Agricultural Experiment Station, Bulletin No. 105. 


\section{DE LAVAL, BABY}

Rated capacity, No. 2, 350; No. 3, 600 pounds per hour

\begin{tabular}{|c|c|c|c|c|c|c|c|c|}
\hline Date. & $\begin{array}{c}\text { No. } \\
\text { of } \\
\text { fac- } \\
\text { tory. }\end{array}$ & $\begin{array}{l}\text { Pounds } \\
\text { of } \\
\text { milk in } \\
\text { whole } \\
\text { run. }\end{array}$ & $\begin{array}{c}\text { Average } \\
\text { temper- } \\
\text { ature. }\end{array}$ & $\begin{array}{l}\text { Range } \\
\text { of } \\
\text { temper- } \\
\text { ature. }\end{array}$ & $\begin{array}{c}\text { Average } \\
\text { speed of } \\
\text { bowl, } \\
\text { revolu- } \\
\text { tions per } \\
\text { minute. }\end{array}$ & $\begin{array}{l}\text { Range } \\
\text { of } \\
\text { speed. }\end{array}$ & $\begin{array}{l}\text { Pounds } \\
\text { separ- } \\
\text { ated } \\
\text { per } \\
\text { hour. }\end{array}$ & $\begin{array}{l}\text { Per cent } \\
\text { of fat in } \\
\text { skimmed } \\
\text { milk. }\end{array}$ \\
\hline $\begin{array}{r}\text { May } 31 . \\
\text { Sep. } 14 . \\
18 .\end{array}$ & $\begin{array}{r}1 \\
14 \\
19 \\
\end{array}$ & $\frac{205}{146}$ & $\frac{96}{83}$ & $\overline{\overline{81-86}}$ & \begin{tabular}{|c} 
Rev. crank \\
54 \\
46 \\
46 \\
\end{tabular} & - & $\frac{362}{303}$ & $\begin{array}{l}.05 \\
.06 \\
.16\end{array}$ \\
\hline Average & .. & $\cdot \cdots \cdot$ & 90 & . & 49 & & 333 & .09 \\
\hline
\end{tabular}

DE LAVAL, STANDARD

Rated capacity, 1,100 pounds per hour

\begin{tabular}{|c|c|c|c|c|c|c|c|c|}
\hline $\begin{array}{cl}\text { Sep. } & 19 . \\
\text { “ } & 19 .\end{array}$ & $\begin{array}{l}21 \\
21 \\
21\end{array}$ & $=$ & $\begin{array}{l}73 \\
73 \\
72\end{array}$ & $\begin{array}{l}71-75 \\
71-75 \\
71-75\end{array}$ & \begin{tabular}{|c|} 
Rev. bowl \\
7,914 \\
8,140 \\
8,300
\end{tabular} & $\begin{array}{l}7,700-8,200 \\
7,600-8,400 \\
8,000-8,500\end{array}$ & $\begin{array}{r}1,020 \\
930 \\
1,000\end{array}$ & $\begin{array}{l}.25 \\
.15 \\
.16\end{array}$ \\
\hline Average & . & $\cdots$ & 73 & . . & 8,118 & $\ldots$ & 983 & .19 \\
\hline
\end{tabular}

\section{SHARPLES RUSSIAN}

Rated capacity, Standard, 1,100 pounds; Imperial, 2,000 pounds peri hour

\begin{tabular}{|c|c|c|c|c|c|c|c|c|}
\hline $\begin{array}{l}\text { July } 16 . \\
\text { July } 16 . \\
\text { Aug. } 17 . \\
\text { Aug. } 24 . \\
\text { Sept. } 14 . \\
\text { Sept. } 17 . \\
\text { Oct. } \quad 6 .\end{array}$ & $\begin{array}{r}2 \\
2 \\
5 \\
12 \\
13 \\
18 \\
18\end{array}$ & $\begin{array}{l}\overline{-} \\
1,718 \\
4,028 \\
2,509 \\
3,562 \\
2,716\end{array}$ & $\begin{array}{l}84 \\
88 \\
80 \\
81 \\
85 \\
87 \\
90\end{array}$ & $\begin{array}{l}83-87 \\
87-88 \\
78-82 \\
80-82 \\
84-86 \\
82-95 \\
87-91\end{array}$ & $\begin{array}{l}7,775 \\
7,183 \\
7,800 \\
7,700 \\
7,433 \\
7,558 \\
7,675\end{array}$ & $\begin{array}{l}7,700-7,900 \\
7,000-7,500 \\
7,700-7,900 \\
7,100-8,300 \\
6,800-7,600 \\
7,200-7,900 \\
7,400-7,900\end{array}$ & $\begin{array}{l}2,100 \\
2,130 \\
1,874 \\
1,033 \\
1,158 \\
1,752 \\
1,873\end{array}$ & $\begin{array}{l}.40 \\
.65 \\
.10 \\
.05 \\
.13 \\
.45 \\
.38\end{array}$ \\
\hline Average & . & $\cdots$ & 85 & $\cdots$ & 7,589 & $\ldots \ldots$ & 1,703 & .31 \\
\hline
\end{tabular}

\section{UNITED STATES}

Rated capacity, 2,000 pounds per hour

\begin{tabular}{|c|c|c|c|c|c|c|c|c|}
\hline $\begin{array}{l}\text { July } 18 . \\
\text { Aug. } 22 . \\
\text { Sept. } 15 . \\
\text { Sept. } 15 . \\
\text { Sept. } 18 .\end{array}$ & $\begin{array}{r}4 \\
10 \\
15 \\
16 \\
20\end{array}$ & $\begin{array}{l}3,962 \\
1,870 \\
3,850 \\
2,902\end{array}$ & $\begin{array}{l}83 \\
81 \\
94 \\
88 \\
78\end{array}$ & $\begin{array}{l}82-84 \\
79-91 \\
90-98 \\
80-100 \\
77-80\end{array}$ & $\begin{array}{l}7,120 \\
7,025 \\
7,600 \\
6,075 \\
6,586\end{array}$ & $\begin{array}{l}7,000-7,200 \\
6,800-7,300 \\
7,200-8,000 \\
5,600-6,600 \\
6,400-6,800\end{array}$ & $\begin{array}{l}2,220 \\
1,964 \\
1,403 \\
1,650 \\
2,176\end{array}$ & $\begin{array}{l}.18 \\
.25 \\
.08 \\
.38 \\
.60\end{array}$ \\
\hline Average & . & . . . . & 85 &.$\cdot \cdot$ & 6,881 & $\cdots \cdots$ & 1,883 & .30 \\
\hline
\end{tabular}


These slight differences in individual bowls are common to all of the different kinds of machines, so far as is known, and this being the case, it is always due to the purchaser that he should secure from the manufacturer a guarantee that any given machine will do work of a certain grade of efficiency.

Other desirable and undesirable features of a separator.-Other things being equal, that separator is the best which will skim clean at the lowest temperature and with the least number of revolutions per minute. Other details of construction being equally good, and the capacities being the same, that separator will run the easiest whose diameter is least. A machine of small diameter not only runs easier, but is less easily thrown out of balance. The cream should be delivered in a smooth stream of uniform density, and the cream outlet should be of such form that a heavy cream may pass through it without danger of clogging. The bowl should be so constructed that all parts may be readily reached with the hand and thoroughly and easily cleaned. 


\section{CHAPTER $X$}

\section{THE RIPENING OF CREAM}

$\mathrm{BY}$ the ripening process is meant all the treatment wilich the cream receives from the time that it is separated from the milk until it is put into the churn. Upon this treatment, and the changes that the cream undergoes, very largely depend the quality of the butter as regards texture and flavor. The texture of the butter is very largely influenced by the changes of temperature that are brought about during the ripening process. It seems to be essential to the production of a firm, solid texture in the butter that the cream, at some time during the ripening process, should be subjected to a constant temperature below $50^{\circ} \mathrm{F}$. for several hours. When cream has been separated by a gravity process, particularly by a "deep setting" one, it has already experienced the effects of such a temperature, and is ready for ripening as soon as removed from the milk. But when cream is separated by a centrifugal separator, the temperature as it comes from the separator is rather high, and butter of good texture cannot be made unless the cream is cooled down and held cool for several hours before ripening has far advanced. The first step, then, in the ripening process with separator cream is to reduce its 
temperature as rapidly and as uniformly as possible to at least $50^{\circ} \mathrm{F}$., and to hold it there for as long a time as is convenient, usually six to eight hours. Another point which influences the texture of the butter depends upon the rapidity with which the various changes of temperature are made, and the extremes of temperature that are used. That butter will have the best texture which has seen the fewest possible changes of temperature between the time the milk is drawn from the cow and the time it is churned, and in which also all the necessary changes of temperature have been made most gradually. Not only will such butter have the best texture at low temperatures, but it will stand the effects of high temperatures better. In other words, it "stands up" under the heat better than butter that during the process of manufacture has been subject to sudden and great changes of temperature, although the final result may have been to keep it at a low temperature. The effects of ripening are more important and more marked upon the flavor of the butter than upon the texture. It is during the ripening that the characteristic flavors of the butter are largely brought out. It is not necessary to the manufacture of the butter itself that the cream be ripened at all. Butter may be made from eream just as soon as it is separated from the milk, but it will be of a distinctly different quality from that made from ripened cream. By ripening in the ordinary sense is meant the production of lactic acid in the cream.

In some attempts to substitute other acids for 
lactic acid in ripening, Tiemann* found that in cream to which a small amount of hydrochloric acid had been added there was no difficulty in churning the butter, that the general flavor of the butter was good, but that it lacked in aroma and had a somewhat oily texture.

There is some doubt as to the origin of the characteristic flavors which are developed in the cream during the ripening process. These flavors are undoubtedly due to the presence of certain volatile substances-fats, bacterial products, or ether-like compounds-which are formed during the ripening process. It was formerly supposed that the production of the characteristic flavors was almost wholly a process of oxidation, and that cream, in order to be properly ripened, and to develop the best flavors, must be supplied with an abundance of oxygen in pure air during the process. Our knowledge of the presence of germs in milk and cream and the effect of their growth upon the various constituents of the milk, has led us to modify these views. At the present time it seems probable that the growth of germs which produce lactic acid has much to do with the production of the characteristic flavors of ripened cream. It has been asserted by some, notably Conn, that the production of the flavors is due to the growth of specific flavor-producing germs that are largely independent of the formation of lactic acid, but this view does not seem to explain all of the phenomena of the appear-

*Milch Zeitung, zxiii p. 701. 
ance of such flavors, and it is by no means certain that the flavors are not in part produced as the result of direct oxidation. It is found in practice that the regulation of the production of lactic acid is the chief means in controlling the flavor.

The means of producing lactic acid. - In order that the milk or cream should ripen, or become sour, it is necessary that germs of lactic acid fermentations should gain access to it, and that a temperature favorable to their normal development should be secured. The presence of the germs may be left to chance inoculation, or they may be artificially supplied. Under ordinary conditions, by the time the cream has been separated from the milk, there will have reached it a sufficient number of germs of fermentation to cause a rapid production of lactic acid, though the number will vary from day to day and from time to time, and a certain amount of acid cannot be depended upon within any given specified time. The inoculation is more certain, and the desired degree of acidity will be more surely reached, at the end of a given time, if the germs are added in sufficient quantity artificially. The source of the inoculation may be buttermilk or cream from preceding churnings, or it may be in the form of an artificially prepared "starter" of sour skimmed milk, or it may be in the form of any of the so-called commercial lactic ferments. It is desirable that none but the proper germs should find access to the milk, and in relying upon natural means there is always more or less danger that putrefactive and other undesirable 
ferments may gain access to the cream. As between the various forms of artificial starter, there is not much to be said. It is generally held that a starter made from skimmed milk is less likely to contain germs other than those desired than when cream, whole milk, or even buttermilk are used.

With regard to the use of the various commercial bacterial ferments, there is considerable diversity of opinion. Their use was first recommended several years ago in Denmark, as a result of the researches of Storch, and has grown rapidly, both in Denmark and Germany, until at the present time a large part of the butter produced in the former country is made from cream first pasteurized, and then ripened with the aid of an artificial bacteria culture. The use of such ferments has now largely extended to this country, also chiefly through the medium of Hansen's lactic ferment. .

In this country Conn has been the chief investigator of similar organisms. From 1890 to $1893^{*}$ he isolated several organisms, the culture of which in cream improved the flavor of the resulting butter to a greater or less extent. In the latter year he discovered in a can of milk sent from Uruguay to the World's Columbian Exposition at Chicago, a germ so much better in this respect that it alone has since been used, and cultures of it placed upon the market under the name of B.41 (Bacillus No.41). A series of investigations by Farrington and Rus-

*Storrs Agricultural Experiment Station, Reports 1890-93. 
sell, ${ }^{*}$ in which a large number of samples of butter were made from cream ripened by the use of B. 41 , and in the ordinary way, or "normally," and submitted to the judgment of several experts who were ignorant of the process of manufacture, led to the conclusion that the "Conn culture, B. 41, did not improve the flavor of the separator butter ripened for one day at a high temperature, or of that ripened for a longer time at a lower temperature; on the contrary, the score of the fresh B. 41 butter by the different judges was, in the majority of cases, materially lower than that of normal butter. *** With separator butter in cold storage, that made with B. 41 deteriorated less than did the normal butter. When taken from storage there was but little difference in flavor between these two butters, although the normal butter when fresh scored higher."

The difference in the flavor of the two kinds of butter when fresh, as indicated by the different judges, is shown in the table:

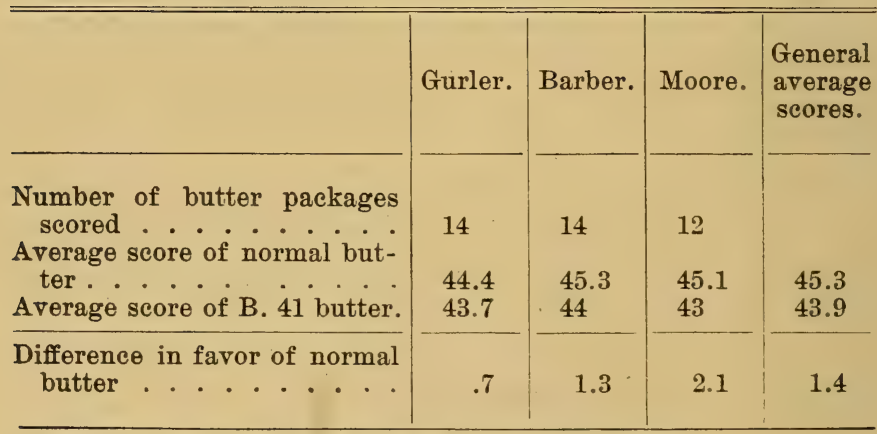

*Wisconsin Agricultural Experiment Station, Bulletin 48. 
On the other hand, a considerable number of factories in this country have secured an increased flavor and quality in their product from ripening their cream with the aid of either Hansen's ferment or B. 41 .

The use of culture starters both for the ripening of cream and the ripening of milk for cheese-making has now become almost universal, particularly in factories, and practice in the culture of such starters is a necessary part of the training of all successful butter and cheese makers (see Appendix A). Skill in the culture of starters depends very largely in keeping everything surrounding them bacteriologically clean, so that, no matter how skilfully directions for the propagation of starters may be written, very few will learn except by actual experience the necessity for bacteriological cleanliness.

The only reason for a starter becoming impure or going "off flavor" is that it becomes contaminated from some outside source, and this comes from lack of care in handling it or allowing it to come in contact with some person or thing that is not sterile or "bacteriologically clean;" hence, in tasting or otherwise examining a starter from which a culture is afterward to be made, a portion should always be removed from the starter with a elean spoon or other suitable article, and such portion must be discarded after the examination has been made. The practice of dipping the finger into a starter and then tasting or smelling it is the best possible means of destroying it.

Where a starter is properly and carefully propagated, it is not only possible to keep it for a long time, 
perhaps indefinitely, but such a starter will often improve in flavor qualities, particularly if propagated continuously in clean, sound, fresh, whole or skimmed milk. Many butter makers employ to advantage the practice of cultivating continuously in suitable small glass vessels several "mother" starters, from which they make selections from day to day of the best flavored for use in ripening.

The use of starters in ripening has another advantage in that it makes it possible to first pasteurize the cream before ripening and so get rid of or reduce the effect of any undesirable fermentations that may be already present. This is of particular advantage where many lots of cream from many sources are brought to a central factory for churning. Such creams vary very much, even where, as is not often the case, none are already actually contaminated with some actually bad fermentation. By pasteurization and ripening with a good, pure culture starter, such creams can be made into butter of a good, uniform quality that would be entirely impossible without pasteurization.

The amount of starter used will vary from 2 to 50 per cent. Where a small amount is used, the purpose is to start and encourage the development of lactic acid through the whole mass of cream. Hence some time must elapse before the full effect of the starter is secured. Where a large amount is used, the purpose is not so much to encourage the formation of the acid as it is to impart directly the flavor already developed in the acid of the starter itself.

The purer, the fresher, and the better flavored the 
cream is, the smaller the amount of starter it is desirable to use. Large amounts of starter should be used only in old, unsound or tainted creams.

Temperature of ripening. - The various germs of lactic fermentations find their optimum growth point at from $80^{\circ}$ to $90^{\circ} \mathrm{F}$., and milk or cream kept at those temperatures will most rapidly become sour. The effect of such high temperatures, while favorable to the production of lactic acid, is less favorable to the texture of the butter, and on this account it is desirable to ripen the cream at as low a temperature as will insure a fairly rapid growth of the lactic germs. A temperature of from $60^{\circ}$ to $70^{\circ} \mathrm{F}$. will ordinarily bring this about. The amount of acid developed in any given length of time will depend not only upon the temperature at which the cream has been kept, but also upon the number and activity of the germs originally present, so that if we have a thorough inoculation to start with, a lower temperature will be sufficient to cause the development of the requisite amount of acid; but if only a slight inoculation is present, a higher temperature will be necessary. Ordinarily, there will be more germs present in the atmosphere during the warm months than in the winter, consequently a lower temperature will bring about the same degree of acidity in a shorter time in summer than in winter.

The amount of acid necessary. - The amount of acid that it is desirable to have in the cream at the time of churning depends, of course, largely upon the flavors desired by the consumer. It is important 
that whatever amount is desired should be uniform from day to day. By far the larger proportion of consumers prefer the flavors that are characteristic of ripening till sufficient acid to slightly coagulate the casein is present. Consumers of delicate taste readily distinguish between the flavors due to a greater or less amount of acid, and it is highly desirable that some means of determining the amount of acid present be available. The butter-maker of trained senses has little difficulty in distinguishing by the somewhat thickened and glistening appearance of his cream, when stirred, the proper condition of the cream for churning. Besides this, there are, fortunately, more exact means for determining the acidity.

Acid tests.-Dr. A. G. Manns first suggested the use of an alkali of known strength to determine the proper acidity of cream for churning, and devised what is known as Manns' acid test. It simply consisted in neutralizing the acid in the cream with a standard alkali, the standard used being that known to chemists as decinormal, or ${ }^{\mathrm{n}} \mathrm{x}$, , and the acidity was reckoned in terms of cubic centimeters of alkali required to neutralize the acid in a given quantity of cream. In the use of this test, an indicator which changes color according as the medium is acid or alkaline, is used. A convenient indicator is phenolphthalein, which is colorless in acid solutions and pinkish in alkaline. Later, Professor Farrington* devised tablets containing a known amount of alkali

*Bull. 32, Ill. Agr. Exp. Sta. Bull. 52, Wis. Agr. Exp. Sta. 
for the same purpose, known as Farrington's alkaline tablets. Each tablet contains an amount of alkali equal to 3.8 cubic centimeters of decinormal alkali, and a sufficient amount of phenolphthalein indicator. The cream to be tested is measured, and to it is added a solution of the tablets (one tablet in ten cubic centimeters of water) until the cream retains a pinkish tinge. The tablet solution should always be fresh, not more than ten hours old. The tablets themselves will keep indefinitely. The proper degree of acidity is indictated when $30-35$ e. c. of Farrington tablet solution, or 11-13 c. c. of decinormal alkali, are required to neutralize the acid in 20 cubic centimeters of cream.

Determination of lactic acid in milk or cream.-Farrington's alkaline tablets may also be conveniently used to determine the percentage of lactic acid in any given sample of milk or cream. In order to do this, it is only necessary to understand that equal volumes of normal or decinormal acids and alkalies neutralize each other; and further, that a normal solution of lactic acid contains 90 grams of acid in each liter, or 1,000 c. c. A decinormal solution would contain 1-10 as much, or 9 grams in each liter, and a cubic centimeter would contain $\frac{1}{1,000}$ as much as a liter, or .009 grams of lactic acid. Each tablet of the Farrington alkali is equal in strength to 3.8 c. c. of decinormal alkali, and if the tablet solution is made by dissolving 10 tablets in $100 \mathrm{c}$. c. of water, each cubic centimeter of the solution will be equal to $.38 \mathrm{c}$. c. of decinormal alkali, and will 
therefore neutralize .38 of .009 grams, or .0034 grams, of lactic acid.

To determine the percentage of acid in any given sample of milk or cream, any convenient amount, as 20 c. c., of the sample to be tested is measured out, and the tablet solution is added from a burette or graduated cylinder till the milk retains a pinkish tinge. If this occurs when 1 c. c. of the tablet solution has been added, the 20 c. c. of milk contain .0034 grams of lactic acid; but 20 c. c. of milk weigh 20.64 grams (sp. gr. milk, 1.032), therefore the percentage of lactic acid is $.0034 \div 20.64=.00017$, or .017 per cent. If more than 1 c. c. of the tablet solution is required to neutralize the acid, or if more or less than 20 c. c. of milk or cream are taken, the percentage of acid will be found by multiplying the number of cubic centimeters of tablet solution required by .0034 and dividing by the number of grams of milk taken (grams of milk $=$ c. c. $\times 1.032)$.

Fig. 31.

B u :

Further effects of ripening.-Besides affecting the flavor, it is believed that ripening aids in the ease of churning, in the completeness of churning, and in improving the keeping quality of the resulting butter. It was formerly supposed that sweet cream could not be churned into butter, and, in fact, with but a moderate percentage of fat ( 15 to 20 per cent) it does churn with much more diffi-

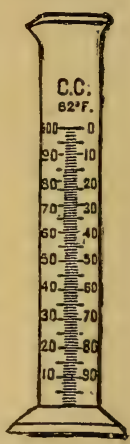

Fig. 32 . Graduated glass cylinder. 
culty than the same cream after it has been ripened; but since the introduction of the separator, and the consequent production of a much heavier cream, it has been found that sweet cream can be churned into butter quite as readily as ripened cream. In cream containing but a moderate amount of fat, it is necessary to reduce the temperature from six to eight degrees, in order to churn it sweet. The difference in the keeping quality of ripened and sweet cream butter is likewise not so great as it was formerly supposed to be. In fact, sweet cream butter, if properly made and kept, will in a short time very closely approach the qualities of ripened cream butter.

Effects of churning cream of different degrees of ripeness. - If parcels of cream of different degrees of ripeness are mixed together and then churned, it will be found that the different creams will churn differently. One will produce butter in a shorter time than another, or with less agitation. The consequence is that when this occurs the churn is stopped before the churning is completed, and much fat is lost in the buttermilk. It is a matter of considerable practical importance, so far as loss of fat is concerned, and to some extent also upon the flavor of the butter, that all of the cream churned at one time should be ripened together, evenly and uniformly. If creams of different ages are to be churned together they should be mixed together at least twelve hours before churning, so that the condition of acidity shall be the same throughout the whole mass. 
Bad effects of over-ripening. - When too much lactic acid is developed in the cream the casein is firmly coagulated, and in the process of churning is broken up into minute granules, which become incorporated into the butter in the form of white specks or flakes of casein. Such white specks, besides injuring the appearance of the butter, greatly detract from its keeping qualities, as the putrefactive fermentations soon set up in them and give rise to disagreeable flavors. Danger from this source is liable to be present if the coagulation of the cream has gone so far that any whey has separated. The cream should in all cases be churned before the ripening process has reached this point. It was formerly supposed, and is still generally believed, that the production of an excess of lactic acid in ripening tends to a loss of butter, from the fact that the acid dissolved or "cut" the fat, causing it to disappear. This has been shown, both theoretically and practically, not to be the case. Lactic acid has no appreciable effect upon fat, so far as dissolving or decomposing it is concerned, and cream may be held until the whey has separated to the full extent without any loss in the amount of butter that it is possible to churn from it. The chief evil effect in overripening is in the production of strong and undesirable flavors accompanying the undue production of lactic acid.

Where the cream is made from milk that has been produced under uncleanly conditions, or has been carelessly handled, or where the cream itself has been 
transported for long distances and subjected to many vicissitudes, bad flavors are much more likely to show themselves again, if the ripening process is carried too far, even though they may have been partially removed by pasteurization. Such cream should be watched carefully and churned with a moderate development of acid. 


\section{CHAPTER XI}

\section{CHURNING}

T'HE process of causing the particles of butter fat to unite into masses, so that they may readily be separated from the milk serum, is called churning. Such union of the particles of fat is ordinarily brought about by agitation of the cream in a suitable vessel, called a churn. If milk or cream be agitated at a temperature somewhat below the melting point of butter fat, the particles of fat, as they pass by one another, agglutinate themselves into masses, and, the process being continued, the first formed masses continue uniting, until finally the whole body of fat may be brought together in a practically solid mass. The conditions influencing the separation of fat in this way are, first, the viscosity of the milk; second, the ripeness of the cream; third, the temperature; fourth, the nature of the agitation; fifth, the quality of the globules of fat.

The viscosity of the mill. - The viscosity of the milk affects the churning, in that it tends to keep the particles of fat from moving freely upon one another, and in that the viscous portions of the milk, notably some of the albuminous matter, form a more or less dense layer about the fat globules. 
tending to keep them apart. The more viscous the milk, then, the greater the difficulty with which it will churn.

The ripeness of the cream.-The ripeness of the cream affects the churning, largely because of its effect upon the viseosity of the milk. The production of lactic acid in milk always has the tendency to render it less viscous, and sour milk or cream, therefore, will churn more readily than sweet for this reason. The viscosity of the milk must be distinguished from the thickness of the milk, due to the coagulation of the casein. Souring of the milk renders it less viscous, but at the same time by coagulating the casein renders it thicker and less fluid.

The temperature.-The temperature is the most important condition affecting churning. Whether the particles of fat shall unite as they pass by one another when the liquid is in motion, depends very largely upon their temperature and degree of plasticity. If the temperature is too low, the globules of fat are so hard that when they hit one another they do not stick together. If, on the other hand, the temperature is too high, the effect of agitating the globules of fat is, instead of causing them to unite, to break them up into still smaller globules, and so render the emulsion more permanent. The range of temperature through which the particles of fat may be made to unite is considerable. The extreme limits may be placed at from $46^{\circ}$ to $80^{\circ} \mathrm{F}$. But while butter may be churned from 
milk or cream through this wide range of temperature, the quality of the butter is very seriously affected. The butter is in the best condition when it is churned at such a temperature that the particles of fat unite readily, and when united form firm masses or granules of butter. The temperature at which this desirable end is brought about varies under a wide range of conditions, the most important of which are the following: The individuality of the animal; the period of lactation; the nature of the food of the cows; the season of the year. These conditions are all conditions which affect the melting point of the butter fat. The higher the melting point of the fat, the higher the temperature at which the milk should be churned, and the lower the melting point of the fat, the lower the churning temperature. Any condition which tends to make the butter fat hard will necessarily be followed by a rise in the churning temperature, and any condition which tends to make the butter fat soft will for the same reason be followed by a fall in the churning temperature. It is impossible, then, to name any single temperature which is the best or even the proper one at which to churn; but while there is a considerable range of temperature, which under different conditions may give the best results, still this range is not so wide as the range of temperature at which it is possible to churn, and the limits may perhaps be placed at from $50^{\circ}$ to $66^{\circ} \mathrm{F}$. In general, the lowest temperature compatible with securing butter in a reasonable 
length of time will give butter of the best quality. The lower the temperature at which the butter is churned, other things being equal, the more completely will the butter be removed from the buttermilk, the longer will be the time required for churning, and the less casein will be found in the butter.

The nature of the agitation. - It is generally believed that the best results follow from agitating the cream in such a way that the particles of fat are subject to more or less concussion, though it is not at all necessary that this concussion. should take place. Mere gentle stirring of the cream, if continued long enough, will bring butter, and agitating the cream by passing bubbles of air through it will also cause it to churn. Many churns have been devised which bring about the churning by agitating the cream with floats or paddles, and in the old-fashioned dash churn there was a minimum amount of concussion, the motion being largely that of stirring. Where the agitation is brought about by the use of paddles or stirring instruments, the texture of the butter is usually injured, because of the effect of the stirring motion upon the grain of the first particles of butter formed. The best churns, then, are entirely hollow vessels, barrel or box, which bring about agitation of the cream through concussion of the particles upon the sides of the rotating churn. The churning depends, also, upon the rate of agitation; the faster the motion to which the particles of cream are subjected, the quicker will be the churning. In this respect the 
motion of the particles and the motion of the churn must not be confounded. If the motion of the churn is increased so that sufficient centrifugal force is generated to cause the particles of cream to partake of

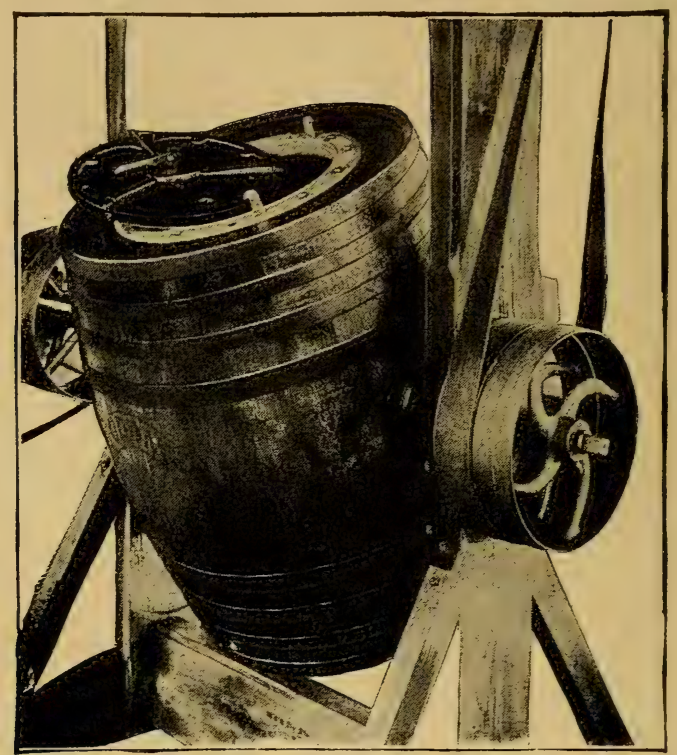

Fig. 33 Barrel power churn.

the motion of the churn, then, though the particles may pass through space at a greater rate of speed, the motion of the particles upon each other will be lessened. A maximum motion of the particles upon each other is obtained when the speed of the churn stops just short of setting up centrifugal force in 
the churn. This will be found to correspond to a motion of the particles of cream of about 700 feet per minute.

The quality of the globules of fat.-The quality of the globules of fat, both as to their size and char-

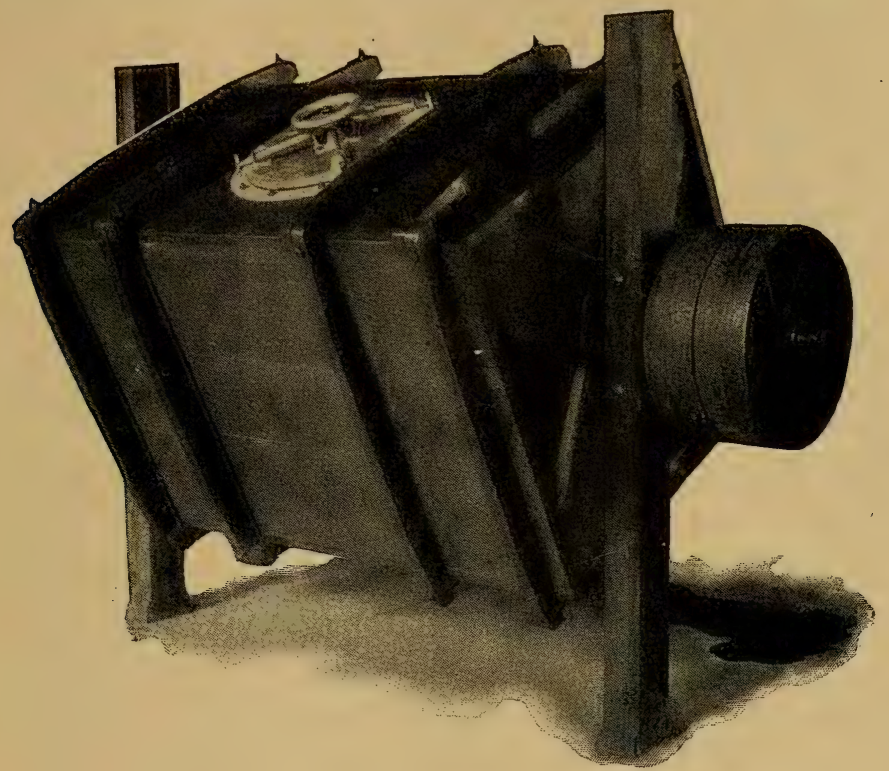

Fig. 34. Square box porver churn.

acter, bears an important part in the phenomena of churning. So far as the hardness or softness of the fats is concerned, this has already been treated of under the effect of temperature; but temperature is not the only influence which causes variation in 
the hardness or softness of the fat. The relative proportions of hard and soft fats vary consider-

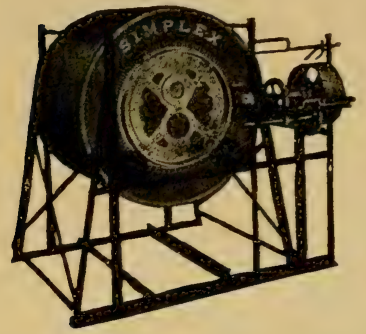

Fig. 35. "Simplex" combined churn and butter worker.

ably. When the soft fats predominate, the churning is easier than when they are in less proportion, while an undue proportion of hard fats often renders churning extremely difficult. The size of the fat globules also bears an important part in the ease and time required for churning. In passing through a mass of liquid two large globules are more likely to hit each other than are two small ones, the relative probability of their meeting and hitting being in proportion to the squares of their diameters.

The end of churning.-When the particles of fat have united to such an extent that they begin to be visible in the cream, the butter is said to "break," and from this time on the process of churning is rapidly finished. Two things are to be observed in bringing the operation of churning to a close. In the first place, the churning should be continued until the separation of fat is as complete as possible. In the second place, the larger the masses of butter in the churning the more difficult is the removal of the buttermilk. If the cream is thoroughly and uniformly ripened, the separation will be more uniform and the churning more complete than when creams of different degrees of ripeness 
are churned together; but under various conditions, and from time to time, the completeness of separation varies with the size of the granules of butter; that is to say, if the granules have reached a certain size, it does not always follow that the fat has been removed from the buttermilk to the same degree, so that the size of the granules of butter is not a certain indication of the completeness of churning. When the churning process is complete, the buttermilk takes on a thin, bluish, watery appearance, quite distinct from the thicker creamy appearance of the unchurned cream, and the churning should be continued until this condition of the buttermilk is reached, even though the granules are increased in size beyond the point favorable to their best separation from the buttermilk. The higher the temperature at which the cream is churned, the greater the percentage of fat left in the buttermilk and the more casein will be incorporated with the butter.

Difficult churning.-Conditions often arise under which it is very difficult or impossible to cause the butter to unite in granules and separate from the buttermilk. One of the chief difficulties accompanies a small and hard condition of the butter granules with a high viscosity in the cream. These conditions usually occur in the late fall and early winter months, when the eows are far advanced in lactation, and when they are often fed on dry food of a character to make hard butter fat. An im'proper ripening of the cream often accompanies these conditions, so that complaints of difficulty in 
churning are usually numerous at this period of the year. These complaints readily yield to the proper treatment, which is indicated under the conditions affecting churning, and so far as is known there never occurs a condition under which it is absolutely impossible to churn the butter from any given sample of cream. The course to be followed in cases of this nature is, first, to add to the food of the cows something of a succulent nature, that will cause the secretion of milk to be greater in amount and not so viscous. Second, to further lessen the viscosity by bringing about a vigorous lactic acid fermentation in the milk, and in extreme instances, perhaps, diluting the cream with warm water or weak brine. Care must be taken that this dilution does not go too far, or difficulty in churning from the thinness of the cream will result. The production of lactic acid in good amounts and within a reasonable time seems to be an important means of overcoming these cases of difficult churning. It is not unlikely that some of the lower fermentations, which take place at temperatures below which the lactic acid germs are active, may in themselves have a retarding effect upon the churning. These cases of difficult churning are frequently accompanied by the production of an acrid or bitter putrefactive fermentation product in the cream.

If the attempt is made to churn at an extremely low temperature, the agitation of the cream serves to incorporate with it bubbles of air, and the cream froths or swells. When this takes place, 
little can be done except to allow the cream to stand for several hours and then warm it up gradually four or. five degrees before again starting the churn. There is very much less danger of swelling in the revolving churns than in dash churns.

Frequently also, in cases when the attempt is made to churn at the extreme lowest point possible, the formation of butter stops just short of the breaking point, and further agitation does not resuit in an increase of butter. This may be overcome by slightly raising the temperature, or by the addition of a little water at a temperature of $85^{\circ}$ or $90^{\circ} \mathrm{F}$.; or, what is better, it can usually be brought about by the addition of a little dry salt to the churn. The solution of salt in the water of the cream seems to affect the viscosity in such a way that the formation of butter granules is greatly facilitated.

When ripened cream is churned, there is usually an evolution of gas immediately after the agitation commences. This is gas which has been formed by some of the fermentations going on in the cream, and the gas is liberated within the first five or ten minutes. The temperature also rises two or three degrees during the operation, from the effect of the friction of the particles upon one another. 


\section{CHAPTER XII}

\section{FINISHING AND MARKETING BUTTER}

WHEN the churning process is complete, it still remains to separate the buttermilk from the granules of butter, and to bring the whole into a solid, uniform mass, suitable for consumption and properly flavored with salt. These operations may be performed in a variety of ways. Formerly it was the custom to continue the churning until the butter was in a measurably solid mass, when it was removed from the churn and the buttermilk expelled by pressure, at the same time that the salt was incorporated with the butter. Now the buttermilk is uniformly removed from the butter by washing with water in the churn, and in many instances the salt is incorporated with it during the whole or part of this operation; but the more common practice is to wash the butter in the churn and to work out the surplus moisture and incorporate the salt upon a separate instrument, called a butter worker.

Washing the butter.-The churning should be stopped as soon as the buttermilk is clear and watery. If the churning has been done at the right temperature and the cream properly ripened, this con- 
dition will occur when the butter granules are not larger than kernels of wheat. As soon as the churn is stopped, the addition of a little cold water to the contents of the churn will facilitate the rising of the smaller globules of butter through the buttermilk. The buttermilk should then be drawn off from the bottom of the churn, and the butter allowed to drain thoroughly. After the butter is thoroughly drained, water at a temperature of $45^{\circ}$ to $55^{\circ} \mathrm{F}$. should be added in amounts equal to twothirds the amount of buttermilk withdrawn. The contents of the churn should then be gently agitated, so that the water may come in contact with each particle of butter, but not to such an extent that the particles will compact themselves into larger masses. In about ten or fifteen minutes, this water should be withdrawn and the butter allowed to drain as before, after which the butter should be washed the second time in the same manner. This second water should run away clear, or with a very slight milkiness. If it is at all milky, the butter should be washed again, and the washing continued until the water does run away clear. It is desirable that as little washing be done as possible, and if care is taken in stopping the churning at the right moment, and in draining and washing the butter, two waters will be sufficient. The addition of a small amount of salt to the first washing water will facilitate the removal of the buttermilk, but will not add any perceptible saltiness to the butter. The way in which the washing is done perceptibly affects the quality of the 
finished butter, particularly so far as regards its texture and percentage of water. The smaller the granules of butter when the churn is stopped, and the colder the water used, the more water will remain in the butter without appearing in the form of drops. If the churning has progressed until the butter has formed masses the size of a hickory nut or larger, particularly if the butter is soft, it will be impossible to completely wash the buttermilk from the butter, and the buttermilk must necessarily be removed in working. Such butter will always retain in its flavor traces of the buttermilk so incorporated with it.

The texture of the butter is also affected by the temperature of the wash water. If the butter comes soft, the attempt is usually made to cool it down rapidly by the addition of large amounts of very cold water. The butter can be hardened up in this way, but it will show the effect of sudden changes of temperature afterward much more strongly than if it were cooled down more slowly. Butter fat does not conduct heat rapidly, and to become thoroughly warm or thoroughly cool requires some little time. If, then, butter has been churned too warm, and comes in a soft condition, the washing process should be much prolonged. Use the ordinary amount of water and at the ordinary temperature, but allow the butter to remain in it until it has had sufficient time to thoroughly adjust itself to the changed condition. It can then be taken out and worked without danger of quickly becoming soft again. There is also 
Working Butter.

223

danger of removing some of the more delicate flavoring oils of the butter by the use of too large an amount of very cold water. These flavoring oils are

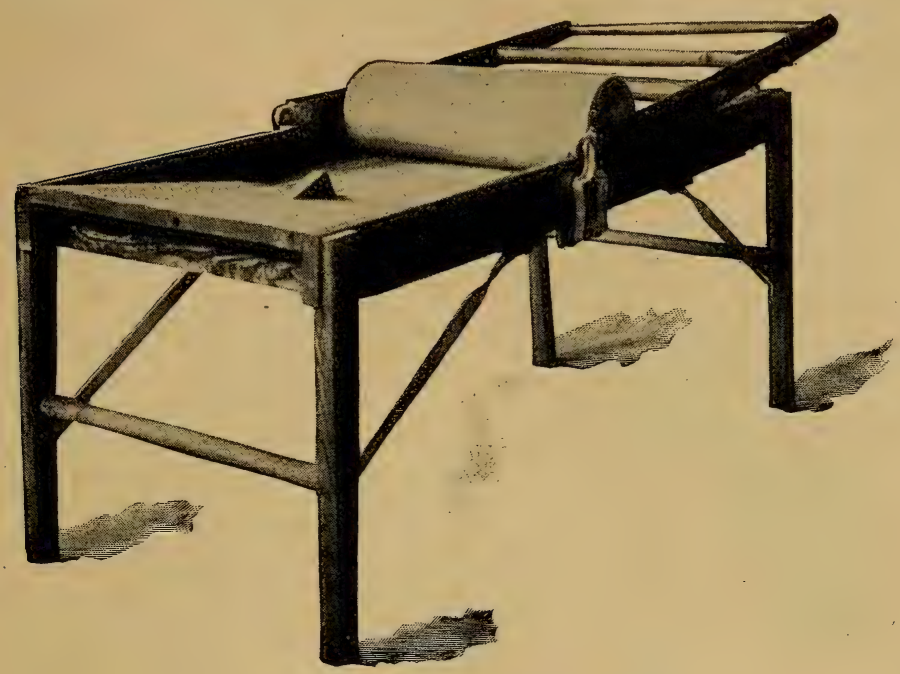

Fig. 36. "Eureka" hand butter-worker.

slightly soluble, and more soluble at a low temperacure, so that the drenching with large amounts of. very cold water has a tendency toward the produceion of a flavorless or tallowy butter.

Working. - Working butter is an integral part of the process of manufacture, only in so far as it relatex to the incorporation of the salt and bringing the butter into the compact form necessary for consumption, so that all working beyond that which is necessary to bring about these two things is super- 
fluous, and, because of the danger of injuring the grain of the butter, is objectionable. The grain of the butter is least affected by working when this is done by pressure and at a temperature of from $45^{\circ}$ to $55^{\circ} \mathrm{F}$, The amount of water that remains

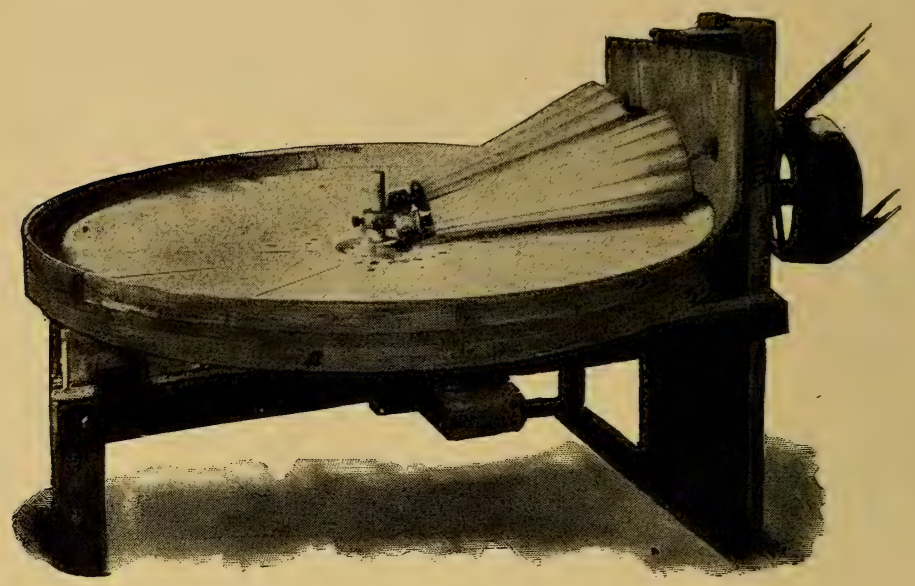

Fig. 37. "Mason" table butter-worker.

in the butter depends, as before stated, upon the temperature and fineness of the granules. The finer and colder the butter, the more water will it retain.

Salting. - Salt is added to butter solely for the sake of the flavor which it imparts. While salt has undoubted antiseptic properties, these play very little part in the preservation of butter, and need not be taken into consideration. The amount of salt, then, that should be added to butter depends entirely 
upon the taste of the consumer for whom it is intended; and may vary from a mere trifle to as much as $2 \frac{1}{2}$ ounces for each pound of butter. The great majority of consumers, however, prefer an amount ranging between $3 / 4$ of an ounce and 1 ounce to the pound. The addition of a uniform

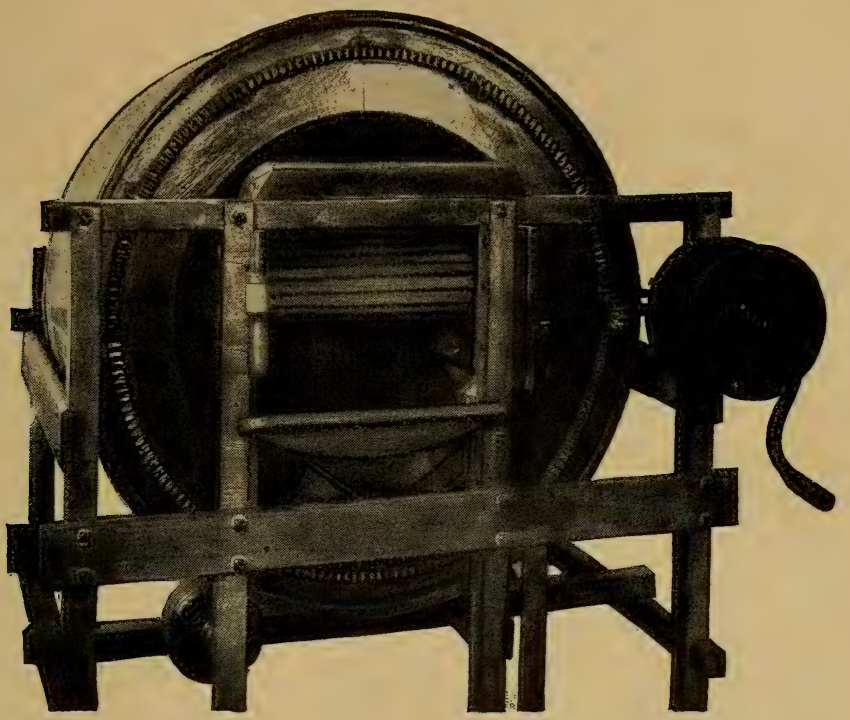

Fig. 38. "Centrifugal" or vertical butter-พvorker.

amount of salt from day to day is a matter of considerable importance in securing a uniform quality of butter, and the amount of salt should, therefore, be based upon the least variable factor possible. It will be found that from day to day the size of the granules of butter when washed, and the o 
amount of water adhering to them, will vary considerably, so that the weight of washed and drained butter will not bear any definite relation to the amount of finished butter, and if the attempt is made to gauge the salt by the weight of drained butter, it will be found that the degree of saltness will vary considerably, because when more water is contained in the butter much more of the salt will pass out with the brine. When a separator is used in creaming, the weight of cream will be found to bear a closer proportion to the weight of finished butter than either the original weight of the whole milk or the weight of the washed and drained butter, and the more uniform salting will be obtained if the salt is added in the ratio of a certain percentage of the weight of the cream. The salt should be added so that it can be readily and uniformly incorporated with the whole amount of butter. This is conveniently done while the water is being expressed from the butter in the worker, and the working should continue until the salt has completely dissolved, because the salt has a deepening effect upon the color of the butter, and if some undissolved portions remain, these, afterwards dissolving in the water contained in the butter, will make a strong brine at that particular point, and consequently a deeper color, and mottled or streaked butter is the result.

The salt should be dry, of uniform grain, and should readily and completely dissolve to a clear solution. Those brands of salt which are made from 
the natural crystal give the best results so far as remaining dry and freedom from caking are concerned.

Brine salting. - With many who prefer a butter slightly salted, it is sometimes convenient to add the salt through the medium of brine, rather than to mix it with the butter in a dry condition. The advantages of brine salting are that a light amount of salt may be uniformly incorporated with the butter. It is not possible to incorporate with the butter a large amount of salt in this way. Where well-drained butter is salted at the rate of one ounce to the pound, there will remain in the butter about 3 per cent of salt. Where butter is washed twice with a saturated brine, there will remain in the butter when worked about 2 per cent of salt. In salting butter in this manner, it is necessary that the brine should be prepared beforehand. This is best done by dissolving the salt in warm water, as much as the water will hold, and then cooling it to the proper temperature to add to the butter. The brine in this instance may take the place of the second wash water. After the brine has remained upon the butter five or ten minutes, it should be withdrawn and additional salt added to it, or a second portion of saturated brine added and left upon the butter for a similar length of time, after which the brine may be withdrawn and the butter worked in the ordinary manner.

Packing and marketing.-After the butter has been worked until the salt is completely dissolved and a sufficient amount of water removed, it is 
ready for packing. Butter that is to be packed and held for any considerable length of time should be worked drier than when it is intended for immediate consumption. Fresh butter is most highly esteemed in most markets when it contains about all the water that it will hold, but if such butter is held for any length of time the evaporation of water from the surface will lead to a deposition of salt, which detracts much from its appearance and sala. bleness. An ideal package for butter is one that is light, strong and tight. None of the packages in ordinary use are perfect in all of these respects. Wooden packages, though light and strong, always leak more or less and let in air. Tin packages are more or less objectionable because of the action of the salt upon the tin, and iron, and glass, and crockery packages, although tight and clean, are heavy and subject to breakage. Packages made of paper or wood pulp have been used to some extent, as have also packages of these materials covered with paraffine, but they do not make a very attractive package. All things considered, tight, smooth, well made tubs of ash, spruce or oak make the most available package. All wooden packages possess the characteristic flavor of the wood, which may be imparted to the butter to a greater or less extent if it is allowed to remain in them very long. In order to obviate this, the odor of the wood should be removed as much as possible before the butter is packed. The package should be thoroughly steamed, and then filled with hot water in which some salt 
has been dissolved, and allowed to stand at least twenty-four hours, after. which it should be scalded a second time and then cooled with cold water. Butter may also be packed in prints or moulds, especially for immediate consumption. The demand for butter in this form is increasing, and for nearby markets it is one of the most desirable forms of packing. In cool weather and with proper precautions, prints may also be shipped considerable distances with success. Prints are usually made in pound and half-pound sizes, intended to go directly upon the table in the shape in which they leave the manufacturer. Various shapes and sizes of prints have been made, but since the great increase in the demand for butter in this shape, a standard size for pound prints has been settled upon that makes a rectangular print, $4 \frac{5}{8} \times 2 \frac{1}{2} \times 2 \frac{3}{8}$ inches. These prints are wrapped in parchment paper and packed in specially made earrying boxes.

Composition and quality of butter.-The average composition of butter is about as follows:

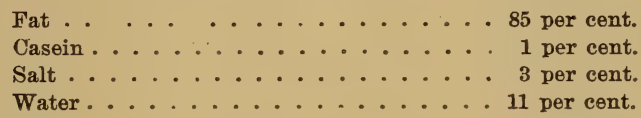

The percentage of fat should not fall below 80 per cent nor the water rise above 15 per cent. The percentage of casein should not exceed 4 per cent. The percentage of fat in butter of good quality often rises to 86 or 88 per cent. The quality of butter is judged upon its flavor, texture, color, amount of salt, 
and its general style and appearance. Butter of good flavor should have the characteristic flavors well pronounced. It should be free from any rancidity, and clean and pure so far as any extraneous flavor is concerned. The determination of the flavor of any sample of butter is entirely one of individual judgment. To some a high flavor is one in which the flavoring oils are developed almost to the verge of rancidity; in others, high flavor means a certain amount of the flavor of sour milk or buttermilk; with still others, that butter is esteemed of the best flavor that is cleanest and purest. The true bouquet of high-class butter is a mild, distinct, volatile flavor or combination of flavors extremely difficult to describe, and only met with in perfection under the best conditions.

The texture of butter depends upon the state of the granular condition of the fats. When the butter is first formed in the churn it makes its appearance in the shape of minute irregular granules. In the subsequent process of manufacture these granules never completely lose their individuality, and constitute the so-called grain of the butter. The more distinctly the individuality of the granules is marked in the mass of butter, the better the texture. The texture of the butter is shown by an appearance like broken cast iron when a mass of butter is broken in two transversely, and when a metal is passed through the butter, as a knife or trier, if the butter be of the best texture, no particles of fat adhere to it. The texture of the butter is deteriorated if the particles of butter are churned in too large masses, and if 
in the process of working the individual particles are made to move upon one another at too high a temperature. The mere warming of the butter to a point approaching the melting point destroys the grain upon subsequent cooling, even though the mass of butter may have been undisturbed.

The color of the butter should be a clear, bright, golden yellow, such as is naturally yielded by the cow when feeding upon fresh pasture grasses. The natural color of the butter is affected very largely by the race of the cow, by the character of the food, and by the period of lactation. Scarcely any breed of cows will yield a butter of ideal color upon dry food late in their period of lactation. On the other hand, one or two races upon fresh pasture will yield a butter of too high a color to suit the most critical trade. The great mass of butter made is artificially colored. For this purpose a large variety of substances has been used, but the coloring matter most commonly employed is a preparation of annatto (a coloring matter in the seed pulp of Bixa orellana) in some neutral oil. This coloring matter is prepared of such strength that only minute quantities are necessary to be added to the cream to produce butter of the proper shade. Aniline colors are also quite largely used in the preparation of butter colors. They are cheaper and do not so easily fade as annatto, and some of them are harmless; but because of the liability of their containing injurious or poisonous substances their use is not to be recommended. Butter artificially 
colored should be uniform, of a bright golden yellow color, free from any reddish tinge. The demand of different markets varies considerably in regard to the shade of color desired.

In regard to salt, the quality of the butter is not necessarily affected by the amount of salt, that being established by the demand of the trade for which it is intended, but the salt, in whatever degree added, should be completely dissolved, and should be uniform throughout the whole mass of butter. The finish of the butter, in whatever form packed, should be such that it will present a neat, clean, attractive appearance. The different qualities of butter are ranked in importance in accordance with the following scales:

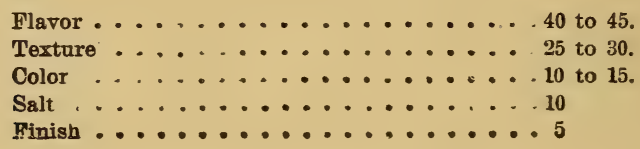




\section{CHAPTER XIII}

\section{MILK FOR CHEESE MAKING}

CHEESE is a product manufactured from milk, into which a large part of the solids are gathered together in such form that their nourishing qualities are retained, at the same time that they are brought into a condition capable of preservation and convenient for transportation. The solids which are concerned in the manufacture of cheese are the casein, the fats, and a considerable portion of the ash. The albumin, nearly all of the sugar, about one-third of the ash, and a small part of the fat, escape during the course of manufacture. The elimination of a large part of the water is the chief step in the process of cheese making. In general terms, cheese consists of about equal proportions of water, fat and casein, with a certain amount of salt and minute quantities of milk sugar and ash. In milk, the easein exists in a state of minute suspension or semi-solution. The essential step in securing the separation of the water is in rendering the casein insoluble. Casein, like most albuminous organic substances, exists in two forms, known respectively as the soluble and the insoluble or coagulated form, and the change from solution to insolubility is termed coagulation. In the case of 
casein, coagulation may be brought about by several reagents, but the one universally employed in cheese making is a soluble ferment found in the stomachs of young mammalia and in certain other animals, known as rennet. By the addition of rennet to milk, the casein takes on the form of a homogeneous gelatinous solid, and in changing its form encloses in its mass the globules of fat. In bringing about the change in the casein in this way, the rennet acts by contact; that is, its own constitution is in no way disturbed, and a minute amount of rennet is capable of causing the coagulation of a large amount of milk.

Quality of milk for cheese making.-While it is scarcely necessary to demonstrate that milk is valuable for butter making in proportion to the amount of fat it contains, the proposition that its percentage of fat is also a measure of the value of nearly all milk for cheese making has not been so readily accepted. Indeed, until within a very short time, the prevailing opinion among dairymen and cheese-makers has been that a milk poor in fat was likely to be rich in casein, and hence more valuable for cheese making purposes. But both fat and casein are constituents of cheese, and both are of nearly equal importance; hence, the richer a milk is in fat, the more cheese it will make, and recent research has shown that for milks containing a normal amount of fat the yield of cheese will be nearly proportional to the percentage of fat in the milk. 
Van Slyke, * in a series of investigations extending over several years, and including the milk of not less than fifteen hundred different cows, found that as the percentage of fat increased the percentage of easein increased in a nearly constant ratio; or, to quote his own words: "While we have noticed considerable variation when we considered individual herds, we have found that, as a rule, there were twothirds of one pound of casein for each pound of fat in the milk, whether the milk contained 3 or 4 per cent of fat; though this normal relation was considerably affected in the season of 1893 by the effects of drought upon the pastures. **** When the amount of fat in the milk increased beyond $4 \frac{1}{2}$ per cent, there was a gradual but slight diminution of casein for each pound of fat."

The accompanying tables give the data upon which Dr. Van Slyke's opinions were based:

Table showing relation of fat to casein in normal milk

\begin{tabular}{c|c|c|c|c|c}
\hline Group. & $\begin{array}{c}\text { Per cent of } \\
\text { fat in milk. }\end{array}$ & $\begin{array}{c}\text { Number of } \\
\text { samples. }\end{array}$ & $\begin{array}{c}\text { Average per } \\
\text { cent of fat in } \\
\text { each group. }\end{array}$ & $\begin{array}{c}\text { Average per } \\
\text { cent of casein } \\
\text { in each group }\end{array}$ & $\begin{array}{c}\text { Average } \\
\text { pounds of } \\
\text { easein for } \\
\text { each pound of } \\
\text { fat. }\end{array}$ \\
\hline I. & 3 to 3.5 & 22 & 3.35 & 2.20 & 0.66 \\
II. & 3.5 to 4 & 112 & 3.72 & 2.46 & 0.66 \\
III. & 4 to 4.5 & 78 & 4.15 & 2.70 & 0.65 \\
IV. & 4.5 to 5 & 16 & 4.74 & 3.05 & 0.64 \\
V. & 5 to 5.25 & 7 & 5.13 & 3.12 & 0.61 \\
\hline
\end{tabular}

*New York Agricultural Experiment Station, Bulletin, No. 68, New Series. 
Table showing relation of fat in milk to yield of cheese

\begin{tabular}{r|c|c|c}
\hline Group. & $\begin{array}{c}\text { Per cent of fat in } \\
\text { milk. }\end{array}$ & $\begin{array}{c}\text { Pounds of green } \\
\text { cheese made from } 100 \\
\text { lbs. of milk. }\end{array}$ & $\begin{array}{c}\text { Pounds of green } \\
\text { cheese made for one } \\
\text { pound of fat in milk. }\end{array}$ \\
\cline { 2 - 3 } I. & 3 to 3.5 & 9.14 & 2.73 \\
II. & 3.5 to 4 & 10.04 & 2.70 \\
III. & 4 to 4.5 & 11.34 & 2.73 \\
IV. & 4.5 to 5 & 12.85 & 2.71 \\
V. & 5 to 5.25 & 13.62 & 2.66 \\
\hline
\end{tabular}

Babcock* tabulated the results of a large number of observations in factories in Wisconsin in the accompanying tables, and while agreeing in the main with Van Slyke, was forced to conclude that, "at the same season of the year, rich milks do not yield as much cheese in proportion to the fat they contain as do poor milks, but that a rich milk toward the end of the season may do as well as a much poorer milk earlier in the season."

Table showing yield of cheese in Wisconsin factories according to per cent of fat in milk

\begin{tabular}{c|c|c|c|c|c}
\hline \hline $\begin{array}{c}\text { No. of } \\
\text { groups. }\end{array}$ & $\begin{array}{c}\text { No. of } \\
\text { reports. }\end{array}$ & $\begin{array}{c}\text { Range of fat } \\
\text { per cent. }\end{array}$ & $\begin{array}{c}\text { Average per } \\
\text { cent of fat. }\end{array}$ & $\begin{array}{c}\text { Average } \\
\text { yield of } \\
\text { cheese per } \\
\text { 100 lbs. milk. }\end{array}$ & $\begin{array}{c}\text { Lbs. of cured } \\
\text { cheese for } \\
\text { 1 lb. fat. }\end{array}$ \\
\cline { 2 - 5 } 1 & 24 & under 3.25 & 3.126 & 9.194 & 2.941 \\
2 & 90 & $3.25-3.50$ & 3.382 & 9.285 & 2.730 \\
3 & 134 & $3.50-3.75$ & 3.600 & 9.407 & 2.613 \\
4 & 43 & $3.75-4.00$ & 3.839 & 9.806 & 2.562 \\
5 & 46 & $4.00-4.25$ & 4.090 & 10.300 & 2.512 \\
6 & 20 & over 4.25 & 4.447 & 10.707 & 2.407 \\
\hline All groups. . & 347 & $\ldots$. & 3.64 & 9.566 & 2.628 \\
\hline
\end{tabular}

*Wisconsin Agricultural Experiment Station, 11th Annual Report, p. 137. 
Table showing yield of cheese in Wisconsin factories by months

\begin{tabular}{|c|c|c|c|c|}
\hline Month. & $\begin{array}{l}\text { No. of } \\
\text { reports. }\end{array}$ & $\begin{array}{l}\text { Average per } \\
\text { cent of fat. }\end{array}$ & $\begin{array}{l}\text { Average } \\
\text { yield of } \\
\text { cheese per } \\
100 \text { lbs. milk. }\end{array}$ & $\begin{array}{l}\text { Lbs. of cured } \\
\text { cheese for } \\
1 \text { lb. of fat. }\end{array}$ \\
\hline April $\ldots \ldots$ & 22 & 3.480 & 9.154 & 2.630 \\
\hline 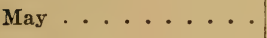 & 68 & 3.493 & 9.447 & 2.704 \\
\hline June $\ldots \ldots$ & 66 & 3.497 & 9.367 & 2.679 \\
\hline July . . . . . . & 63 & 3.554 & 9.231 & 2.593 \\
\hline August $\ldots \ldots$ & 49 & 3.634 & 9.335 & 2.568 \\
\hline September..... & 36 & 3.836 & 9.955 & 2.594 \\
\hline 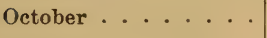 & 28 & 4.076 & 10.562 & 2.591 \\
\hline November $\quad \ldots$. & 15 & 4.254 & 10.947 & 2.573 \\
\hline Whole season ... & 347 & 3.64 & 9.566 & 2.628 \\
\hline
\end{tabular}

Table showing yield of cheese for one pound of fat for each month

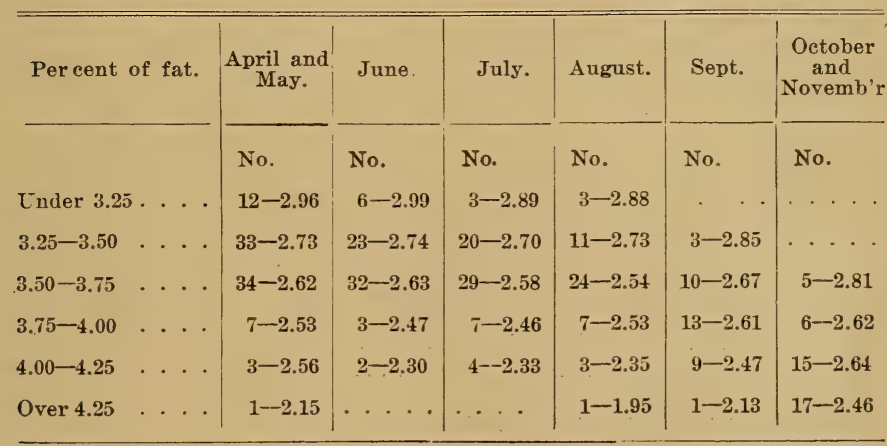

On the other hand, Dean* has found that uni-

* Ontario Agricultural College, 21st Annual Report, p. 44. 
formly a rich milk yields less cheese in proportion to the fat contained in it than a poor milk. His experiments covered a whole season's work in a single factory, the average results of which are shown in the following table:

Field of cheese from rich and poor milk

\begin{tabular}{|c|c|c|c|c|c|}
\hline & \multirow{2}{*}{$\begin{array}{l}\text { Num- } \\
\text { ber of } \\
\text { experi- } \\
\text { ments. }\end{array}$} & \multicolumn{2}{|c|}{$\begin{array}{c}\text { Per cent of fat in } \\
\text { milk. }\end{array}$} & \multicolumn{2}{|c|}{$\begin{array}{l}\text { Pounds of green cheese } \\
\text { for one pound of fat } \\
\text { in milk. }\end{array}$} \\
\hline & & $\begin{array}{l}\text { Richer } \\
\text { milk. }\end{array}$ & $\begin{array}{l}\text { Poorer } \\
\text { milk. }\end{array}$ & $\begin{array}{l}\text { Richer } \\
\text { milk. }\end{array}$ & $\begin{array}{l}\text { Poorer } \\
\text { milk. }\end{array}$ \\
\hline April $\ldots \ldots$ & $\begin{array}{l}6 \\
6\end{array}$ & $\begin{array}{l}4.21 \\
\cdots\end{array}$ & 3.39 & $\begin{array}{l}2.66 \\
\therefore \cdot\end{array}$ & 2.86 \\
\hline $\operatorname{May} \ldots \ldots$ & $\begin{array}{l}15 \\
15\end{array}$ & 4.09 & 3.30 & 2.68 & 284 \\
\hline June $\ldots \ldots$ & $\begin{array}{l}12 \\
12\end{array}$ & 3.94 & 3.16 & 2.68 & 2.94 \\
\hline July $\ldots \ldots$ & $\begin{array}{l}14 \\
14\end{array}$ & 3.78 & 3.00 & 2.73 & 3.06 \\
\hline August. . . . . . & $\begin{array}{l}13 \\
13\end{array}$ & 3.82 & 2.91 & 2.74 & 3.14 \\
\hline September. . . . . . . & $\begin{array}{l}12 \\
12\end{array}$ & $\begin{array}{l}4.01 \\
.\end{array}$ & 3.06 & 2.68 & 3.06 \\
\hline October . . . . & $\begin{array}{l}13 \\
13\end{array}$ & $\begin{array}{c}3.99 \\
\cdots\end{array}$ & 3.26 & 2.82 & 3.05 \\
\hline Average. . . . & & 3.98 & 3.15 & 2.71 & 2.99 \\
\hline
\end{tabular}

The practical bearing of the relation of the fat to the casein arises in the question as to whether, where milk is bought for cheese making, it should be paid for by weight or according to its percentage of fat. It does not require proof, that to pay an equal price per pound or hundred weight for milk of all qualities is a gross injustice to those 
who produce the richer milks. If there were a quick, accurate method of determining casein, the logical practice would be to pay for the milk upon the basis of its known content of both fat and casein; but failing in that, it is well to examine in how far we may fall back upon the percentage of fat, the determination of which is both quick and accurate, as a measure for determining the value of milk for cheese making. While there is some disagreement, it seems to be pretty well settled that a milk rich in fat is also richer in casein, and with milk of ordinary quality, including probably nine-tenths of all the milk produced, nearly in proportion to the percentage of fat. So that, even if the richer milk does yield a little less cheese for each pound of fat, the percentage of fat is a much more nearly just measure of its cheese-producing power than is the weight of the milk, the measure now commonly employed. Further, when the milk is so rich in fat that the casein falls considerably below its normal ratio to the fat, the resulting cheese will be so much richer in fat that it will be of considerably better quality, and such milk, when pooled with milk poor in fat, will so bring up the quality of the whole product, that it should be paid for at a correspondingly higher rate. The conclusion, therefore, is that the percentage of fat is by far the most accurate measure at present available for the determination of the cheese-producing value of milk, and that milk is practically valuable for cheese making in proportion to the amount 
of fat that it contains, and should be paid for accordingly.

Loss of fat in cheese making. - It has been a generally accepted idea by cheese-makers, that the necessary loss of fat in the whey is much greater when the milk contains a high percentage of fat. Indeed, the statement has been frequently made that all the fat in the milk above 4 per cent is lost in the whey. This idea has had much to do with the disinclination to value milk for cheese inaking according to its percentage of fat, and, in fact, has been a chief argument in favor of the manufacture of skimmed or partly skimmed cheese.

The following table by Van Slyke* shows that this idea is erroneous, and that the loss of fat in the whey need be proportionately no greater when the milk is rich than when it is poor in fat.

Table showing amount of fat lost and recovered in making cheese

\begin{tabular}{|c|c|c|c|c|c|}
\hline $\begin{array}{l}\text { Number of } \\
\text { experi- } \\
\text { ment. }\end{array}$ & $\begin{array}{l}\text { Pounds } \\
\text { of fat } \\
\text { in } 100 \\
\text { lbs. } \\
\text { of milk. }\end{array}$ & $\begin{array}{l}\text { Pounds of } \\
\text { fat lost in } \\
100 \mathrm{lbs} \text {. } \\
\text { of milk. }\end{array}$ & $\begin{array}{l}\text { Pounds of fat } \\
\text { recovered in } \\
100 \text { lbs. of } \\
\text { milk. }\end{array}$ & $\begin{array}{l}\text { Pounds of fat } \\
\text { lost in whey } \\
\text { from } 100 \mathrm{lbs} \text {. } \\
\text { of fat in } \\
\text { milk. }\end{array}$ & $\begin{array}{l}\text { Pounds of fat } \\
\text { recovered in } \\
\text { green cheese } \\
\text { from } 100 \mathrm{lbs} \text {. } \\
\text { of fat in milk. }\end{array}$ \\
\hline 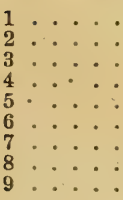 & $\begin{array}{l}2.35 \\
3.01 \\
3.88 \\
3.96 \\
4.70 \\
4.73 \\
4.80 \\
6.49 \\
3.70\end{array}$ & $\begin{array}{l}0.154 \\
0.193 \\
0.277 \\
0.283 \\
0.359 \\
0.331 \\
0.373 \\
0.715 \\
0.269\end{array}$ & $\begin{array}{l}2.196 \\
2.817 \\
3.603 \\
3.677 \\
4.341 \\
4.399 \\
4.427 \\
5.775 \\
3.431\end{array}$ & $\begin{array}{r}6.55 \\
6.42 \\
7.15 \\
7.14 \\
7.64 \\
6.99 \\
7.77 \\
11.01 \\
7.26\end{array}$ & $\begin{array}{l}93.45 \\
93.58 \\
92.85 \\
92.86 \\
92.36 \\
93.01 \\
92.23 \\
88.99 \\
92.74\end{array}$ \\
\hline
\end{tabular}

Other investigators have abundantly confirmed these results.

"New York Agricultural Experiment Station, Bulletin No. 37, p. 681. 
Cooling.-All that was said in Chapter VII on "market milk," in regard to cleanliness in drawing the milk, applies equally well to milk that is intended for cheese making, but where milk is made into cheese it is not necessary, $110 r$, indeed, is it desirable, that the lactic acid fermentations be checked further than is necessary to allow the milk to come into the cheese maker's hands in the best condition. In cheese making, the fermentations of the milk play a most important part, and while it is not at present known just what fermentations are necessary or most desirable, sufficient is known to assure us that a certain amount of lactic acid is not only desirable but necessary for the cheese making process, and enough further is known that those fermentations which attack the protein substances, and result in the formation of strong flavors, taints and gases, are all undesirable in cheese making. For the purpose of cheese making, it is desirable that the fat should separate from the other constituents of the milk as little as possible, and to that end the previous treatment of the milk should be such that the separation of cream will be held back as mueh as possible. One of the best means of doing this is to cool the milk to the temperature of the air while it is kept constantly in motion. This, beside helping to keep the cream mixed with the milk, will also favor the escape of any volatile animal products that may be present in the milk, known under the collective name of animal odor, and which would otherwise impart objectionable flavors to the cheese. 
Aëration.-Passing the milk through an elevated strainer in such a manner that it may fall in finely divided drops or streams through the air before it reaches the can, is one of the best means of bringing about this end. This is known as

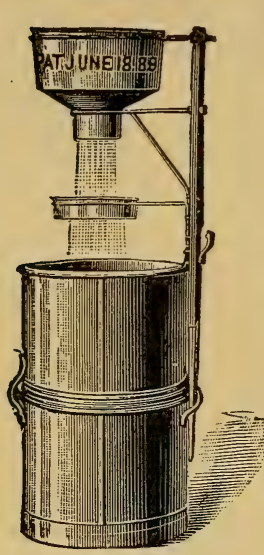

Fig. 39. "Hodgkin" elevated strainer. aëration. It cannot be depended upon to destroy or even check the germs of fermentations which may be present in the milk, beyond that due to the reduction of temperature. It is not desirable that milk intended for cheese making should be cooled much below the temperature of the air, and one of the best preparations of milk intended for cheese making is to allow it to remain where it will be freely exposed to a pure and moderately cool atmosphere. Under ordinary conditions, the milks of the evening and the succeeding morning are manufactured together, and the treatment indicated is naturally that of the night's milk. It is highly desirable, however, that the milk of the morning should be cooled to the temperature of the atmosphere before it is taken to the factory, and under ordinary conditions the milk of the night and morning should be taken to the factory in separate cans.

Ripening.-The lactic acid fermentation is probably one of the most important factors in all the steps of cheese making, and to know to what ex- 
tent it has progressed before the process begins, is exceedingly important to the cheese maker. To this end, several convenient tests may be applied. The veteran, who has attained skill through long manipulation, can tell with a fair degree of accuracy through the sense of smell and taste the amount of lactic acid present in his milk, but this is not a safe enough guide for those who are inexperienced, or those who expect to make a uniform product in a uniform way from day to day.

- Rennet tests.-The percentage of lactic acid present in the milk can readily be determined directly by titration with a standard alkali, but the amount of lactic acid present is also quite readily. determined by the length of time required for rennet coagulation, since rennet acts more quickly upon milk the riper it is, and upon this fact two or three simple tests have been devised. Fig. 40. Monrad rennet test. $a, 5$ c. c. pipette; The simplest of these $b, 50$ c. c. gr

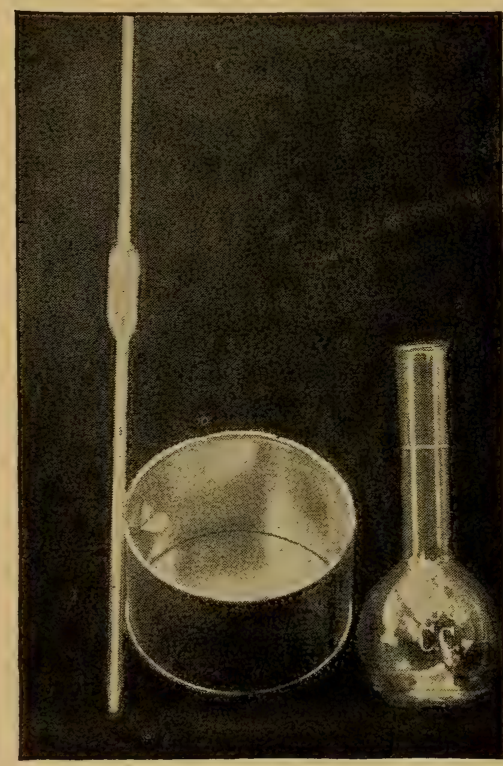

b $b, 50$ c. c. graduated flask; $c$, cup in which 
is to add a spoonful of rennet of known strength to a teacupful of milk and, after stirring the two together thoroughly, to note carefully the time required for coagulation. Another and more exact method, known as the Monrad test, is made as follows: To a measured quantity of milk at a given temperature a measured quantity of rennet of known strength is added; ordinarily the commercial rennet is diluted nine times for this purpose. The rennet is thoroughly stirred with the milk, and the time required for coagulation is carefully noted. This is simply a development of the cup test, and is better because of its greater delicacy and accuracy; the milk and rennet being more carefully measured, and, diluted rennet being used, a wider range of delicacy

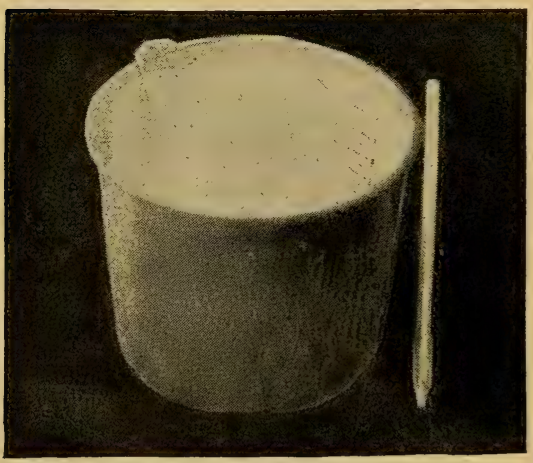

Fig. 41. Marschall rennet test. is reached. Still another form of rennet test, known as the Marschall test, has been devised. In this test the milk is measured into a vessel, one of whose sides is graduated and in the bottom of which there is a minute orifice. The rennet is added to the milk, and the vessel so placed that the milk can escape from the hole in the bottom. So long as the milk rèmains 
fluid the stream is continuous, but as soon as the rennet takes effect and a clot is formed, the stream stops, and no more escapes. The number of spaces of milk which escape is taken as the measure of the ripeness of the milk. When the percentage of lactic acid is great the stream stops sooner than where little acid has been formed, so that in the ripe milk a less number of spaces shows upon the side of the vessel than in the unripe milk.

Degree of ripeness necessary.-By ripeness of milk for cheese making is meant, as in the case of butter making, the degree of lactic acid development that has taken place. For cheese making, the development of lactic acid should not have proceeded so far that the milk is distinctly sour to the taste, and it should have proceeded far enough so that the whole process of cheddar cheese making, from the time of adding the rennet to putting the cheese in press, will take not more than six hours. This will be indicated by a rennet test of $2 \frac{1}{2}$ spaces on the Marschall apparatus, or a rennet test of one minute on the Monrad test, when 5 c. c. of diluted commercial rennet extract ( 1 part rennet to 9 of water) are added to 200 c. c. of milk.

Starters. - In very many cases the milk, when brought to the factory, will not be sufficiently ripe to give the tests indicated above. In that case it should be allowed to ripen before the cheese making process begins. This may be brought about by heating the milk up to $85^{\circ}$. F., when the germs already present become more active; but the time 
required for the ripening will be shortened, and more uniform results be obtained if, in addition to the warming, an artificial starter is used. This may be any material containing the germs of lactic fermentation in active condition. The whey saved from the previous day, or milk naturally or artificially soured, may be used. An extremely convenient form of starter is made by preparing an artificial ferment in the following way: Ten pounds of whole milk are sterilized at $180^{\circ} \mathrm{F}$., then cooled to $90^{\circ}$, and sufficient commercial dry lactic ferment added to secure coagulation in twenty-four hours. When coagulated, this is added to the extent of 10 per cent to enough whole milk to make sufficient starter for one day's use. (See Appendix A.) The amount of starter to be added for the purpose of ripening the milk should be from 2 to 5 per cent, varying with the temperature of the air and the amount of ripeness that it is necessary to develop after the milk reaches the factory. Enough of the ferment should be reserved each day to prepare ferment for the following day from whole milk; and with care that the vessels in which the ferment is made are kept clean and sweet, a single ferment may be propagated for from ten days to two weeks. As a matter of fact, there is no reason why a ferment may not be propagated for an indefinite time. All that is necessary is to keep everything bacteriologically clean and to prevent infection by never allowing any dust or solid particles to fall into the ferment and never to touch it with the hands. Such a ferment will give more uniform results, 
and is less liable to introduce taints and bad flavors, than a ferment made from sour whey, and with a little care and attention, the fermentation will go on regularly from day to day.

Rennet-The ordinary source of rennet is the fourth or digestive stomach of a calf that has not yet ceased to live upon milk, though rennet is also found in the stomachs of puppies and pigs, and the latter have occasionally been used as a source of rennet. The active principle of the rennet is found in the folds of the lining membrane of the stomach of the animal, and is greatest in amount when a full meal is just digested.

Rennet causes coagulation of the casein by contact, and is not itself affected by the change. Its action, however, is greatly modified by various conditions of the milk, the most important of which are the temperature and the condition of certain of the ash constituents. Rennet is most active at temperatures near the body heat, $98^{\circ} \mathrm{F}$. At temperatures below $80^{\circ} \mathrm{F}$, the action of rennet is correspondingly slow. On the other hand as the temperature is raised above $100^{\circ} \mathrm{F}$., the activity of the rennet is at first increased, but after about $130^{\circ} \mathrm{F}$. is reached the active principle is rapidly weakened and finally destroyed at about $140^{\circ} \mathrm{F}$.

The activity of rennet is also intimately connected with the lime salts in the ash of the milk, and particularly with their condition as to solubility. The presence of soluble salts of lime renders rennet much more active, while the insoluble salts of lime have 
a correspondingly retarding effect. Thus, the development of lactic acid by rendering the lime salts more soluble hastens the action of rennet on the milk. On the other hand, when milk is heated to a temperature above $150^{\circ} \mathrm{F}$. a part of the lime salts is made insoluble, and the action of the rennet correspondingly retarded, even though the milk be again cooled to a temperature at which rennet is active. The coagulability of milk is similarly affected by the addition of any lime salt not normally present in milk. Thus, if calcium oxalate or other insoluble. salt of lime is added to milk, the action of rennet is retarded. On the other hand, the addition of calcium chloride or other soluble salt is followed by a quicker action of the rennet.

The rennet skin should be obtained from an animal of some age. Still-born calves, or calves less than three days old, furnish a rennet of very inferior quality. The calf should be slaughtered three or four hours after it has had a full meal, and the fourth stomach carefully taken out. This should be emptied by turning inside out and any particles of foreign matter carefully picked off; it should not be washed with water. It should then be sprinkled with salt and stretched in such a manner that it will readily dry.

At the present time the preparation of the rennet extract from the skins is entirely a commercial one, and rennet so much superior, on account of its uniform strength and freedom from taints, is furnished in this way that it is not advisable to prepare 
the rennet for use from the skins. In the preparation of the extract, the skins are soaked in warm water to which a little salt has been added until the active principle has been completely removed from them. The resulting extract is filtered, and preservatives, in the shape of salt and a little saltpetre, are added. Rennet so prepared will retain its strength and purity for a considerable length of time, if kept in a cool, dark place.

Removal of whey.-After the coagulation of the milk is complete its constitution is as follows: 92.5 parts soluble, 7.5 parts insoluble. The soluble portions are made up of water 87, sugar 4.5, albumin .75 , ash .25 . The insoluble are made up of casein 3.25 , fat 3.75 , ash .5 . The separation of these two is the chief task of the cheese maker, and is brought about partly by mechanical and partly by chemical or physical agents. The chief mechanical agents in the separation are cutting the curd, stirring, and the application of pressure. The chemical and physical agents in effecting the separation are: First, application of heat; second, development of lactic acid; third, addition of salt; fourth, curing fermentation. These various agents may be applied in various degrees and in various ways, all of which have a greater or less effect upon the character of the resulting cheese, producing an almost endless variety of product. The process that we shall describe is one in common use in America, although first practiced in England. It it known as the American Cheddar process, and is a modification in minor de- 
tails of the process first employed at least 250 years ago, in the district about the village of Cheddar in Somerset, England, from which it takes its name, and from which it has spread more or less over the whole world. Cheddar cheese is still made in Somerset, as well as in other parts of Great Britain, notably Ayrshire. It is with minor variations the process most commonly employed in the United States, Canada and Australia, and one of its distinctive features, namely the matting of the curd after the whey is drawn, is used in making many other varieties of cheese, as the Cheshire, Leicestershire and Derbyshire in England, the Edam and Gouda in Holland, and perhaps others. 


\section{CHAPTER XIV}

\section{CHEDDAR CHEESE MAKING}

THE successive steps in the manufacture of Cheddar cheese may be conveniently grouped into seven stages or periods, as follows: Period first, setting; period second, cutting; period third, heating; period fourth, cheddaring; period fifth, grinding; period sixth, salting and pressing; period seventh, curing.

Period I., setting. - The rennet is most active at temperatures from near that of the body, $98^{\circ}$ to $100^{\circ} \mathrm{F}$., up to about $130^{\circ} \mathrm{F}$, and if added to the milk at these temperatures will most quickly cause coagulation (p. 245), but at high temperatures there is more tendency of the fat to separate, and for this reason the milk is warmed to a degree sufficient to cause fairly rapid coagulation, and at the same time not injure the fat. This will vary from $82^{\circ}$ to $86^{\circ} \mathrm{F}$., according to conditions. The milk, when brought to the factory, is collected in the vats and the whole mass gradually warmed up to the proper temperature, with frequent gentle stirring to prevent any separation of the fat in the form of cream. When the whole mass is warmed up to $82^{\circ}$ the milk is tested for ripeness, and if sufficiently ripe the rennet is added at once, but if the milk is insuff- 
ciently ripe a starter is then added, and the milk allowed to stand at this temperature until a sufficient amount of acid has developed, care being taken that it is frequently stirred and no cream allowed to rise.

The rennet should be added in sufficient quantity to cause the milk to coagulate in from ten to fifteen minutes, and to be ready for the knife in from thirty to thirty-five minutes. It was formerly supposed that the amount of rennet added had an effect upon the whole process of manufacture, and particularly upon the curing fermentation, but while the matter is still unsettled, later researches go to show that the influence of the rennet upon the curing is probably very slight. The amount of rennet to be used will also vary with the strength of the extract; two to three fluid ounces of rennet, of ordinary strength, per thousand pounds of milk is sufficient. The rennet should be added to the milk in such a way that the coagulation will be uniform throughout the whole mass. If the rennet is added in full strength and at a high temperature, the milk will be immediately curdled as soon as the particles of rennet come in contact with it, and coagulation will begin in part of the mass before the rennet can be thoroughly united with the whole body of milk. The rennet should be diluted with twenty to fifty times its own bulk of cold water. The dilution renders the uniform mingling of the rennet with the milk easier, and the cold water keeps it inactive until it has been warmed up to the 
temperature of the milk. So soon as the rennet is added, the milk should be quickly and carefully stirred, so that the rennet may be uniformly mingled with every part of it. This stirring should continue until the mixture of rennet and milk is complete, but should stop before any appearance of coagulation. The milk in the vat is then allowed to become quiet, and remains undisturbed while the process of coagulation is going on, the object being to secure a uniformly solid clot or coagulum through the mass. The action of the rennet is not instantaneous; the first appearance of coagulation is noticed by a slight thickening of the milk. This gradually increases until the whole mass is solid, and if left undisturbed the action of the rennet continues to contract the coagulated casein and cause a partial separation of the whey. As soon as the coagulation is strong enough, so that the casein will maintain its shape when broken, the contents of the vat are ready for the next step in the process.

Period II., cutting.-The curd, as the coagulated casein is called, is ready for cutting when under strain it will break with a clean fracture. This is conveniently tested by gently inserting the finger in the curd in an oblique position and slightly raising it, when if the curd breaks clean across the finger it is sufficiently firm for cutting. Cutting is performed in order to facilitate the further contraction of the casein and the expulsion of the whey. Formerly the solid mass of curd was broken up into small pieces by any sort of an instrument that 
might be at hand, or simply with the fingers, no care being taken that the pieces of curd should be of uniform shape or size, but now gangs of steel knives, in one of which the blades are arranged horizontally and the other perpendicularly, are used to cut the mass of curd into cubes of about $3 / 8$ of an inch in diameter. The fat is retained in the cheese by being enclosed in the meshes of the casein, and in breaking up the mass of casein, a certain number of fat globules is always set free. The eare with which the cutting and subsequent handling of the curd is done controls the loss of fat through this source. Formerly, the mass of curd was allowed to become very firm before cutting, but the more recent practice has shown that the best results in expelling the whey from the curd are reached when the curd is cut, so soon as it has sufficiently coagulated to maintain its form. The curd is cut by passing the horizontal knife through the vat, usually in a longitudinal direction, and then by passing the perpendicular knife both longitudinally and crosswise of the vat. It is immaterial whether the horizontal or perpendicular knives are used first. Some skilled cheese-makers prefer the one practice and some the other. The object to be attained is to break up the curd into as uniform a mass of kernels as possible, or, in the language of the cheese-maker, to secure an "even cut." After the curd is cut, the whey begins to escape, and the curd sinks to the bottom of the vat. If allowed to remain undisturbed, the cut surfaces of the particles 
of curd readily unite, and in breaking them apart again more fat is lost. Hence as soon as the cura is cut, gentle agitation of the whole mass should begin. This agitation should be sufficient to cause the particles to move upon one another, but not violent enough to break them up. The curd rapidly shrinks and hardens; more rapidly upon the outside than the inside. This soon results in the formation of a so-called "membrane," which not only tends to prevent the particles from sticking together, but affords some resistance to further breaking up. This "membrane" is pervious to water, but retains the globules of fat; so as soon as the contraction has reached this point, or when the curd is well "healed over," the further expulsion of the water should be aided by heat, and this point begins the third step.

Period III., heating or cooking.-The term "cooking," as applied to the manufacture of cheese, is a misnomer so far as it relates to any change in the composition or condition of the material through the effect of heat. By cooking any ordinary substance is meant the application of a degree of heat sufficient to cause a coagulation of albuminous substances or a breaking down of starchy ones. The degree of heat used in cheese making during this stage never reaches anywhere near this point, and never, except in rare instances, is a temperature of blood heat exceeded. The change to be brought about in this process is wholly the contraction of the curd and the consequent expulsion of water from within the particles. To this end the heat serves a two- 
fold and important part. The curd is contracted to some extent by the action of the rennet still continuing; also to some extent by the direct application of the heat, but to a greater extent by the effect of lactic acid upon the curd, the production of lactic acid being greatly favored by the increased temperature. It is desirable that this contraction of the curd in the cooking stage go on uniformly throughout the whole mass. The heat is therefore applied gradually and with constant stirring. As the particles of curd shrink in size, the tendency for them to unite in masses constantly diminishes, and they are broken with greater difficulty, so that while at first extreme care is necessary to prevent the particles of curd from becoming broken, as the temperature rises, the stirring may become more rapid and vigorous. The heat should be raised slowly frọm the setting point of the milk up to $98^{\circ} \mathrm{F}$, and it should not be more rapid than $2^{\circ}$ in five minutes; and unless the lactic acid develops very rapidly it is better to take even more time than this. When the temperature of $98^{\circ} \mathrm{F}$. is reached, the stirring may cease and the particles of curd be allowed to settle upon the bottom of the vat until the curd is thoroughly shrunken and a sufficient amount of lactic acid has been developed. At the end of the cooking stage the particles of curd should have shrunken to less than one-half of their former size, and should have become so hard that when pressed together between the hands, and the pressure suddenly relaxed, they will fall apart, and show no 
tendency to stick together. By this time there should have developed a sufficient amount of lactic acid so that the curd will draw out in fine threads a quarter of an inch long when applied to the hot iron.

When the curd has reached the highest temperature, it may be allowed to settle, and a part of the whey may be drawn off the top of the vat. This is of advantage particularly when the development of lactic acid is likely to be rapid, and it is desirable to separate the whey from the curd in a very short time, but sufficient whey should be left to cover all of the curd two or three inches deep until sufficient acid has been developed for the cheddaring process.

Period IV., cheddaring, or matting. - When sufficient acid has developed in the whey, it is drawn off from the vat through the gate or by means of a syphon. The curd is then allowed to mat together into a mass, which is the distinctive feature of the cheddar process. This matting together may be done either in the vat or in a special vessel, called a curd sink. In the former case, when all the whey is run off the particles of curd are brought together upon the two sides of the vat and a channel opened between them, so that any whey held by the particles of curd may be allowed to escape. When the particles of curd are sufficiently matted together to maintain themselves intact, the mass is eut up into blocks about $8 \times 8 \times 12$ inches, and these blocks are turned over so that 
the part that was uppermost comes in contact with the bottom of the vat. A further draining of the whey takes place, and the blocks of curd are next piled upon one another two deep, care being taken in forming these piles that the parts that were exposed to the air are turned in. Later on the curd is piled again in still deeper piles, and as the process continues the mass is piled over and over again, care being taken that the exposed parts of one pile are put into the interior of the succeeding, so that the heat may be uniform throughout the whole mass. In the curd sink the manipulation is not essentially different. The curd sink is a square wooden receptacle fitted with a false slatted bottom and covered with coarse cotton or linen eloth, through which the whey can easily escape. When the time comes for separating the whey, so much as will run off readily is drawn off, and the remaining whey, mixed with particles of curd, is dipped into the sink, the whey runs off freely through the strainer and slatted bottom, and the curd, being spread over the whole surface of the sink, soon mats into a solid mass, and the piling process goes on the same way as is done in the vat. During this process various changes take place. The pressure of the particles of curd upon one another serves to expel a large part of the whey that still remains; at the same time the temperature is kept sufficiently high so that the production of lactic acid is not checked. The effect of the acid is to cause a series of marked changes 
in the physical condition of the curd. From the condition of a tough, spongy mass when first cheddared, the curd changes into a smooth, elastic, fibrous condition, not unlike the physical appearance of well cooked, lean meat. When the curd reaches the condition above described it is ready for the next step in the process, and by this time sufficient lactic acid will have developed so that fine threads may be drawn out two to three inches on a hot iron.

Period $V$. , grinding. - When the curd is ready for grinding, the whey has been removed to so great

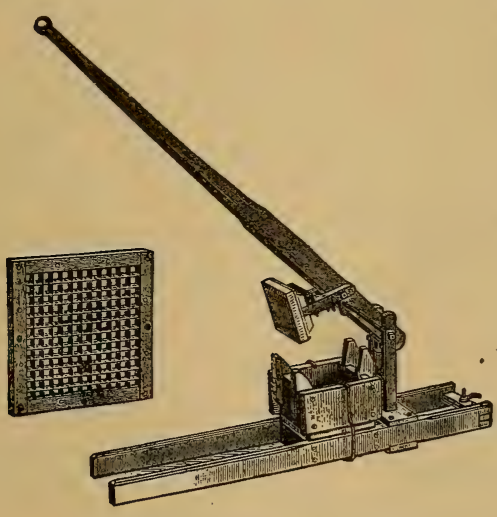

Fig. 25. "Harris" curd mill. an extent that the production of lactic acid measurably stops. The curd is ground in order to reduce it to particles of convenient size for receiving the salt and for pressing into a solid mass in the cheese. The grinding, or more properly the cutting, is done in special machines known as curd mills. Of the various styles of mills, those are best which cut the curd into pieces of uniform size, without tearing it apart, for the reason that when so treated less fat escapes and the uniformity of size of the pieces aids in the proper and even absorption of the salt by the 
curd. After the curd is ground, it is kept sufficiently stirred to keep the particles from matting together

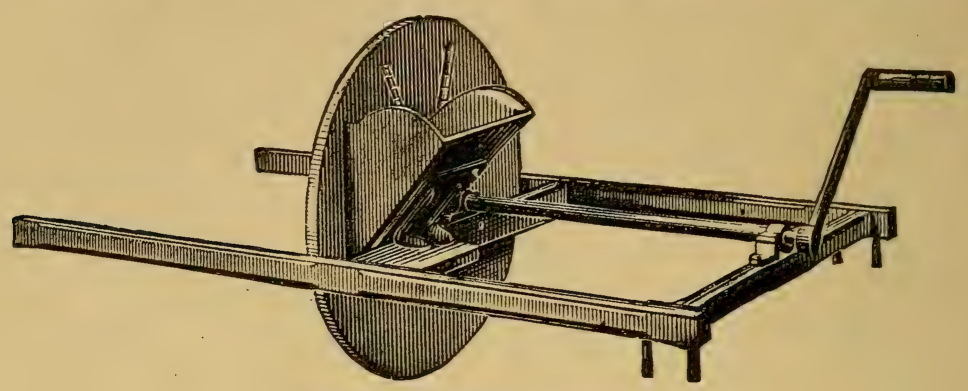

Fig. 43. "MacPherson" eurd mill.

again. A further maturing of the curd takes place, during which it takes on a peculiar nutty flavor, and the particles of casein begin to break down

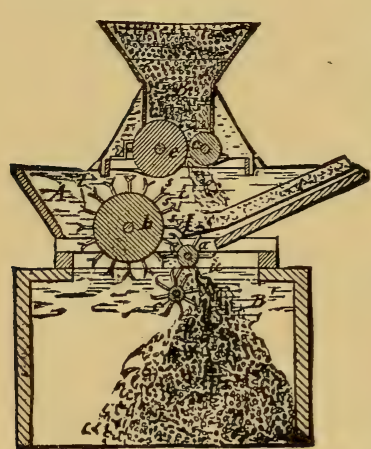

Fig. 44. Section of "Pohl" curd mill. so that the fat may be removed by pressure. When this stage is reached the curd is ready for the next step, which is salting.

Period VI., salting.-Salt is added to the curd primarily for the sake of the flavor it imparts to the cheese, just as salt is added to butter: but the addition of the salt to the curd has a further effect in the process of manufacture. The salt makes the curd drier by reason of extracting the water for its own solution, and at the same time 
hardens the curd. It also checks the further development of lactic acid. The salt should be uniformly mixed through the curd, and the curd kept stirred until it is fully dissolved. A salt of rather coarse grain is preferable for cheese. It requires a somewhat longer time for solution, and the particles of the salt are carried to the centre of the pieces of curd more effectually. When the salt is thoroughly dissolved the curd is ready to put in the press.

During the cheddaring and grinding stage various means, as covering with cloths, etc., have been taken to keep up the temperature of the curd, and at the time when the cheese is ready to salt it should not be below $90^{\circ} \mathrm{F}$. When the salt is added the eurd should be spread out thin, so that it will cool off, and when it is put into the press should be at a temperature of from $78^{\circ}$ to $82^{\circ} \mathrm{F}$. If the curd is put in the press too warm, the fat is more easily pressed out and lost. On the other hand, if the curd is at too low a temperature when put in the press, it is more difficult to make the particles adhere together into a solid mass. The object of pressing the cheese is to bring it into a form suitable for transportation and convenient for consumption: The pressure also removes any surplus moisture that is in the curd; but the primary object of pressing is not to remove moisture. In fact, all of the moisture that is removed from the cheese by the press is that held by capillarity between the particles of curd, and practically none can be expressed from the particles themselves. If the curd is too 
lower than $78^{\circ} \mathrm{F}$, a slight amount of pressure will cause the particles to unite in a smooth and solid mass. The pressure should be uniform and continuous for at least twenty hours. Where a screw press is used, eare must be taken to tighten the screws as rapidly as they become loose, particularly for the first hour after the cheese is put in the press. Those presses that are fitted with appliances for taking up the slack and making the pressure continuous show excellent results in the texture of the cheese. After the cheese has been in the press for three-fourths of an hour it should be taken out, turned, the bandage straightened, and the whole cheese wiped with a cloth wrung out of water as hot as can be borne by the hand. This warming of the surface aids in the formation of a firm, transparent rind, and it improves the appearance of the cured cheese. Seamless bandage is practically the only kind now used, and it should be cut of such a length that it will extend over each end of the cheese for an inch and a half or two inches; when the cheese is put in the press, circular eap eloths should be put between the ends of the cheese and the follower. These cap cloths are allowed to remain upon the cheese after it is taken from the press and while it is curing, and are removed just before the cheese is boxed for market.

Period VII., curing. - The green cheese, when taken from the press, if exposed to a temperature of about $70^{\circ}$ in a pure atmosphere, undergoes a series of fermentations which result in breaking 
down or rendering soluble the casein and in the development of the characteristic flavors peculiar to good cheese. These flavors are almost entirely developed during the ripening process. The ripening is brought about by a series of fermentations that goes on in the cheese. It is not well understood just what these fermentations are; but it seems evident that at least in the early stages of the curing, lactic acid germs are active, although the greater majority of these disappear after a short time. During the ripening process, oxygen is taken up and carbonic acid given off. The quality of the cheese is best when the ripening process goes on gradually and continually. The higher the temperature the faster the ripening will go on, an extreme temperature of $65^{\circ}$ or $70^{\circ}$ giving the best results. At the end of from four to six weeks the casein will be so broken down that the cheese is fairly digestible and fit for consumption, though if kept longer under good conditions the cheese will improve for three or four months, and then if kept moderately cool, and in not too moist nor too dry an atmosphere, it may be kept one or two years.

If the cheese is well made, if the whey has been thoroughly separated in the process of making and if the milk was sound and free from taints, the ripening process will go on regularly even at low temperatures, though the time required is much longer. The resulting cheese will be of the best possible flavor and texture. The practice is now quite general to remove the cheese at the age of seven to ten days at once to cold 
storage $\left(30^{\circ}\right.$ to $35^{\circ} \mathrm{F}$.) and to leave them there for several months till fully ripe. This practice is particularly common with cheese intended for winter or export trade. During the early part of the curing process the cheeses should be turned upon the shelves every day until a sufficient amount of water has evaporated, so that they no longer tend to lose their shape.

Difficulties likely to occur in cheddar cheese making.-The chief difficulty in cheddar cheese making comes from the presence in the milk of germs which produce fermentations that are undesirable These fermentations usually begin during the cook. ing process, and continue through cheddaring. Ordinarily they evolve a considerable amount of gas, causing what are known as floating or gassy curds, and are usually accompanied by disagreeable odors and flavors. The formation of the gas in the curd gives it a sponge-like texture, and when the particles of curd are cut across they are seen to be full of minute holes, the condition usually denominated pin-holes. The best means of treating this trouble is, of course, prevention; but even with the utmost care by makers of experience, milk containing the germs of these fermentations will find its way into the vat.

In many cases the trouble from gassy and tainted milk is caused by the milk of one or two dairies, and in exceptional cases the milk of a single cow may infect a whole vat. The particular dairies or even cows that are causing the trouble may usually be detected by employing one of the fermentation tests 
described in Appendix A. Ordinarily these fermentations do not work with the lactic fermentation; each hinders the action of the other so that the chief means of overcoming the difficulty of gas or pin-hole curds is to favor in every way possible the production of lactic acid. To this end the milk is well ripened before the rennet is added, and the heat is raised as rapidly as possible to a higher temperature of cooking than ordinarily is used. In extreme cases the curds may be heated as high as $104^{\circ} \mathrm{F}$. After the whey has been drawn great care is taken to keep the temperature of the curd from falling, and at the same time the escape of the gas is favored by frequent turning and piling of the curd. Where the curds are gassy the cheddaring process must be continued until the formation of gas has ceased and the holes in the curd have collapsed. In extreme cases, where the gassy curds have produced very strong, undesirable flavors, these may be removed by drenching with hot water. The means used to prevent the development of gas, and to get rid of the gas already formed, ordinarily favor the escape of fat from the cheese, so that while the bad effects of the gassy curd may be largely eliminated from the finished cheese, still it is always done at the expense of a certain loss of fat.

Another difficulty that often confronts the cheesemaker is that coming from the milk arriving in too ripe a condition, not necessarily accompanied by undesirable fermentations. In the case of such milk, the development of lactic acid is very rapid and the 
curds, technically described, "work fast." When this occurs, the production of lactic acid is more rapid than the shrinking of the curd, and the whey is sufficiently acid to be drawn off before the curd has shrunken down. In this case, the curd should be thoroughly and continually stirred after the whey is drawn until the whey has been well worked out before the curd is allowed to cheddar; but where it is known at the beginning of the process that the milk is over-ripe, care should be taken throughout the whole process to use every means to retard the formation of lactic acid, and at the same time to cause as rapid a shrinking of the curci as possible. To this end, the milk may be set at a lower temperature and a larger amount of rennet used, and when the curd is cut it should be știrred until the whey has well separated before the heating process begins. With care in these particulars, there is slight danger of the formation of acid before the curd has shrunken down.

Qualities of cheese. - A well cured cheddar cheese of good quality should have about the following composition:

Per cent.

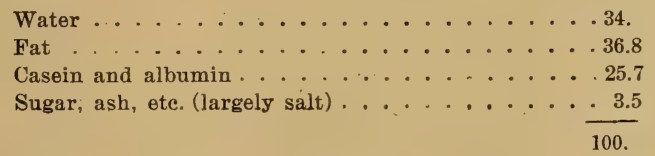

The characteristic flavors should be well pronounced but not strong, depending somewhat upon the age of the cheese. The flavor should also be 
clean; that is, free from any flavors due to the influence of undesirable fermentations or to foreign matters that may have gained access to the milk through the food or otherwise. The texture should be solid, smooth and firm. When bored with the trier, the plug should come out solid, or nearly so, and smooth. There should be no moisture visible in any part, and no appearance of any separation of the fat from the easein. When crushed, the cheese should readily break down into a smooth, unctuous mass, without indication of the presence of undue moisture, and should emit a pleasant, nutty flavor. When broken across, the flaky texture should be manifest, the so-called "flinty break." The color should be uniform, not mottled, and with more or less of a tendency to translucence, especially in new uncolored cheese. The rind should be smooth, without eracks. hard and transparent. The bandage should extend evenly over the ends of the cheese for about two inches and should be straight and smooth. The ends of the cheese should be parallel and the sides straight. These various qualities are usually ranked according to the following seale :

Per cent.

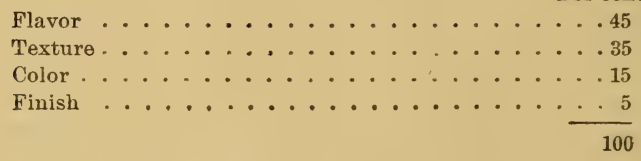




\section{CHAPTER XV}

OTHER VARIETIES OF CHEESE

SLIGHT variations in any of the minor details of the cheese making processes have a distinct influence on the character of the finished product. A natural result of this is that the varieties of cheese are almost innumerable. Von Klenze* in his Hand Book of Cheese Technology describes no less than 156 different kinds, whose manufacture is distributed through Europe and America. Not only is the number of distinct kinds very large, but the same kind or variety varies greatly in character and quality, according to the conditions under which it is made. Without attempting to accurately classify the various kinds of cheese, it may be said that they fall roughly into about three natural groups or classes. First, those whose chief characteristics depend upon the amount of water that has been removed in the process of manufacture. These we may call hard or soft cheeses. Second, those whose qualities depend upon the amount of fat which the cheese contains, whether it is the normal amount of the milk, whether a part of the fat has been removed, as in the case of skimmed or partially skimmed cheeses, or whether fat has been added to the milk as in the case of the so-

\footnotetext{
*Von Klenzє, Handbuch der Kâserei-Technik, Bremen, 1884.
} 
called cream cheeses. And third those whose dis tinctive characteristics depend upon the sort of fermentation to which they have been subjected. The details of manufacture upon which the peculiar characters depend are, in many cases, so intricate and minute that it is practically impossible so to describe them that a novice might successfully follow out the directions. The actual practice must in most eases be learned at the hands of an experienced teacher. We shall, therefore, limit our discussion to a few of the better known and most largely manufactured varieties. The introduction of the so-called fancy varieties in America is in its infancy, but is rapidly developing, and bids fair to become a most important diversification of the dairy industry.

American home-trade, or stirred-curd cheese.-The popular consumptive demand of most American markets requires a softer and milder flavored cheese than the cheddar or export type. This is brought about by incorporating a larger amount of water with the curd, and by hastening the curing process, and not curing it so far as is ordinarily done with a wellripened cheddar. The details of the manufacture of the American home-trade and the American cheddar or export are in the main similar. In fact, the two processes merge into one another in such a way that we find a regular gradation in the cheese from the softest, mildest, short-keeping stirred-curd cheese to the most solid, long-keeping cheddar. The distinctive differences in the two processes of manufacture are that in the home-trade cheese, after drawing the whey 
the curd is not allowed to mat into a mass or cheddar, but is kept stirred in such a way that the whey will drain off until it is dry enough so that the particles of eurd will not unite. It is then salted and, with more or less further maturing of the curd, is pressed and cured. The character of the resulting cheese, however, depends quite as much upon the amount and character of the changes that go on in the curd after the whey is drawn, and before it is put into press, as upon the mere fact of allowing it to pack in a mass (cheddar) or keeping it apart by stirring. If the cheddar variety is ground shortly after matting, and immediately salted and put to press, the resulting cheese will resemble the stirred-curd type. On the other hand, if the stirred-curd is kept warm and frequently stirred, many of the same changes will go on as in the cheddared curd, and the cheese when cured will resemble the cheddar type.

Another distinction between the two processes is that in the case of the cheddar cheese the aim is to get rid of all the water consistent with a compact union of the particles of casein. In the manufacture of the stirred-curd cheese, on the other hand, the aim is to retain as much water as possible without having it appear in the form of free water in the cheese. To this end, in the stirredcurd process those influences which tend to contact the curd, namely, the application of heat and the development of lactic acid, are not carried so far as in the cheddar process. In fact, in many cases they are not carried far enough to remove all of the water, and some remains in the cheese, making a "wet," "sloppy" or "leaky" cheese. 
In order to obviate the difficulties that arise from leaving too much whey in the curd and still make a cheese of soft texture, the practice of "washing" or "soaking" curds has come into use. An ordinary cheddar or stirred-curd is made, making it dry and firm. When ready for the press the curd is immersed in cold water $\left(60^{\circ} \mathrm{F}\right.$.) for fifteen to forty minutes. The water acts to remove bad flavors that may have been present in the milk and the curd swells and absorbs a considerable amount of water. The resulting cheese has a soft melting texture but the flavor is usually deficient or no better than would have resulted from leaving too much whey in the curd.

Because of the larger amount of water it contains, this kind of cheese is profitable to the producer of the milk and to the manufacturer, and when properly made and carefully cured, it may be of excellent quality, being mild, creamy and soft; but largely, too, because of the large amount of water contained in it, it is easily subject to decomposition changes, goes off flavor rapidly, and does not bear transportation well.

Sage cheese. - In many parts of the United States this cheese is very popular for local consumption. Its manufacture is not different from the ordinary type of cheese, either cheddar or stirred-curd, but an infusion of sage leaves, or sage extract (in which latter case the green color is secured by an infusion of fresh leaves of any inert plant, as clover, green corn, etc.), is added to the milk before the rennet coagulation, and imparts a light greenish color and characteristic flavor to the curd. Ordinarily, where sage cheese is made, the sage is 
added to only a part of the milk, and two separate curds are manufactured simultaneously, and mixed together as they are put in the press, resulting in cheese of a mottled green color.

Young America, picnics, pineapple, and truckle cheese.-These are names applied to various forms of small cheeses. The truckle and Young America cheeses in particular are small cheeses of the cheddar or other common type, pressed in sizes of six to ten pounds weight. Pienics are somewhat larger and are usually pressed in 10 -inch hoops and weigh from 20 to 25 pounds. They are usually soft and mild-flavored. The pineapple cheeses are pressed in shapes suggested by their name, and are generally made as firm and solid as possible.

Nenfchatel is a soft uncured cheese, made by coagulating milk with rennet, allowing the resulting curd to become mildly acid and then removing the surplus moisture by drainage and pressure, after which the curd is ground, salted, molded in small cylinders $1 \frac{3}{4}$ inches in diameter by $2^{1 / 4}$ inches long and wrapped first in thin parchment paper and then in tin foil. Good Neufchatel should be soft, smooth and melting in the mouth without "mushiness," due to the retention of too much moisture. When properly made it can be subjected to very heavy pressure without losing its melting texture. Its flavor when fresh is simply the flavor of clean, mildly acid coagulated milk. The successful manufacture of Neufchatel depends upon securing both fine texture and good flavor, for while, as a rule, these two qualities 
are closely connected, it is quite possibie to have good texture with objectionable flavor. It is probable that the same conditions of temperature and germ growth that are most favorable to ripening cream for churning are also most favorable for the manufacture of Neufchatel cheese. At any rate, the milk should have some degree of ripeness before the rennet is added. It is essential that the milk be clean and pure, that the acidity is developed to the proper point in the curd, and that sufficient pressure is applied. When experience in these last two points is gained, the remainder of the process is comparatively simple, and in fact, many of the details may be varied to a greater or less extent. Good clean milk is not less important than in cheddar cheese making, as gassy ferments often make trouble. A good pure-culture starter, giving a mild development of acid, will prove of great assistance in handling gassy milk. The rennet is added to the milk at a low temperature $\left(70^{\circ}\right.$ to $80^{\circ} \mathrm{F}$.) at the rate of $1 \frac{1}{2}$ to $2 \frac{1}{2}$ ounces per 1,000 pounds. Too little rennet will give trouble by making a curd that is very difficult to drain, and that tends to pass through the press cloths when pressed. An excess of rennet results in a curd of hard texture. It is better to add the rennet to the whole mass of milk in a vat, and then as soon as the rennet is thoroughly mixed with the milk, to draw it off into "shot-gun" cans ( 8 inches in diameter and 20 inches deep) for coagulation. The milk should be warm enough and also ripe enough, so that coagulation will take place 
before much cream rises to the surface. The temperature of the room may vary within rather wide limits, though if it is above $80^{\circ} \mathrm{F}$. the curd is likely to be hard and tough, while if it is $60^{\circ} \mathrm{F}$. or below, the curd is not injured, though the process is greatly retarded. Probably the same rule holds here as in cream-ripening, namely, that it is not possible to name a single temperature arbitrarily as the correct one. The most important point in the whole process is to determine when the curd should be dumped from the coagulating eans upon the draining cloths. It should stand until the whey is markedly acid to the taste, as much so as mildly ripened cream, and until the hand passed down between the sides of the can and the curd notes that the latter is firm, with a peculiar elastic cushiony feel. The amount of whey that gathers on the top of the curd is not a safe indication of the time to dump, for at high temperatures a large amount of whey often separates while it is still sweet, and in case of gassy fermentations the curd floats and the whey is found at the bottom. If the cans are dumped before sufficient acid has developed, it will be very difficult to drain the curds, the flavor will be very bad, the texture pasty and slimy, and later, as the acid develops, an additional amount of whey will separate, making a leaky cheese. When ready, the cans of coagulated milk should be dumped on draining racks $15 \times 24$ inches, with slatted bottoms covered with heavy, closely-woven cotton sheeting. The dumping should be carefully and skilfully done, 
so that the curd slides out of the ean with as little breaking as possible. The conditions are best when the mass of curd, on being dumped, retains its form and breaks with a clean vitreous fracture. The time required for drainage may range from two to twenty-four hours, varying with the temperature, amount of rennet used, amount of acidity developed, and other unknown factors. When dry enough to handle easily, the cloths are folded up in such a manner as to enclose the curd, and the whole is pressed under rather heavy pressure till the proper consistency is secured. When taken from the press the cakes of curd are brought to a uniform consistency by kneading or working, or they may be passed through an ordinary meat chopper. About $1 \frac{1}{2}$ per cent of salt is then thoroughly incorporated and the cheese molded into the desired shape. The molding is a matter of some difficulty, as the particles of cheese move easily on themselves, while they tend to adhere to metal or wooden surfaces. Large factories have specially constructed machines for molding the cheese. On a small scale, the most satisfactory implement is a smooth tin tube of the proper diameter and long enough to hold two or three cheeses. The tube is filled full of the curd and then forced out of one end with a smoothly fitting piston, after which the cheeses are cut apart with a thread and neatly wrapped first in thin parchment paper and then in tin foil. According to the quality of milk, from 60 to 80 standard cheeses should be made from 100 pounds of milk. 
Philadelphia or square cream cheese.-This is a soft cheese growing in popularity in proximity to many of our larger markets. It resembles the Neufchatel, but is made of cream of varying degrees of richness and is put up in a different form. It is molded in flat, thin cakes $3 \times 4$ inches, in a flat tin mold, wrapped in parchment paper, and packed for shipment. Its manufacture is practically the same as Neufchatel, and, in fact, there is more or less confusion of nomenclature between the two, as well as a good deal of variation in fat content, some brands of Neufchatel having a higher fat content than some brands of square cream, and vice versa.

Some of the older writers describe a cream cheese made by simply draining and lightly pressing in square blocks a thick cream obtained by setting milk in shallow pans, heating over water till the cream "crinkles," and setting in a cool place for twelve to twenty-four hours.

Limburger. - The Limburger is one of the varieties of cheese whose characteristics are due to specific fermentations brought into the cheese during the ripening process. These fermentations result in a well known putrefactive odor and pungent flavor. According to Monrad,* the manufacture of Limburger in America and Europe is not greatly different. Ordinarily the cheese is made from whole milk, but frequently skimmed or partly skimmed milk is used. In Europe the copper kettle is commonly employed; in America both the kettle and the ordinary rectan-

*Cheese-making in Switzerland. Winnetka, Ill., 1896. 
gular cheese vat are used. In the latter case the curd is made in much the same way as for ordinary cheese up to the point when the whey is drawn off. The milk is set at a rather high temperature $\left(92^{\circ}\right.$ to $\left.100^{\circ} \mathrm{F}.\right)$. The eurd is broken into pieces the size of a hen's egg, and allowed to settle to the bottom of the kettle. It is then scooped out and put in rectangular molds arranged on tables, so that the whey may drain off. The molds are carefully turned till the whey has measurably ceased running, and the cheeses will maintain their form. They are then placed in rows on a flat table with thin pieces of board between them, and subjected to light pressure from the sides. The cheeses are turned frequently at first, and then at longer intervals, till at the end of thirty-six to forty-eight hours they may be taken from the press. They are then salted by rubbing salt on the ends and flat sides for three or four days. After the first salting they are laid on the table in single layers, afterward they are piled, at first two deep, then three or four deep, so that the absorption of the salt may be promoted. During salting and pressing they are kept at a uniform temperature of from $59^{\circ}$ to $63^{\circ} \mathrm{F}$. They are then cured in a cool $\left(60^{\circ} \mathrm{F}\right.$.), well-ventilated cellar nearly saturated with moisture, with careful watching and much manipulation and turning. With the ripening they begin to soften. The curd, at first hard, takes on the characteristic glassy, greasy appearance, at the same time that the rind becomes at first yellow, then reddish yellow. The softening begins on the outside 
and proceeds toward the center, and the cheeses are considered to be marketable when one-quarter of the cheese has taken on its characteristic texture.

Imitation Swiss cheese.-A considerable amount of cheese closely resembling the true Emmenthaler is made in America. Its main distinctive characteristic lies in its peculiar flavor, and in the appearance of the peculiar, so-called Swiss holes in its texture. Both of these are due to specific fermentations, which take place in the cheese during the curing process. (See Emmenthaler cheese, page 289.)

These are the principal varieties of cheese that are manufactured to any large extent in America. There are, however, a number of brands of cheese upon the market that may properly be classed under the general name of

Prepared cheese.-These fancy brands are in general made from an ordinary cheese of good quality, by removing the rind and reducing the remainder to a homogeneous, more or less pulpy, mass. To this is added a certain amount of additional fat, either in the shape of butter or other fat, and some flavoring matter in the form of cayenne pepper, brandy, or something of like nature. The cheese is then packed closely in fancy glass or earthenware packages, sealed tightly, and marketed. The names under which it is sold vary with the manufacturer and with the recipe under which it is made. Many of them are sold under registered trade-marks. Some of the better known are Club House, Meadow Sweet, Canadian Club, etc. 
Among the varieties of cheese of foreign manufacture, the following are worthy of mention:

English cheeses.-The various dairy localities in England produce cheeses bearing their distinctive geographical names. In the main, they are of the cheddar type, and differ from the true cheddar only in details of manufacture and in slight differences in texture and flavor, in much the same way that the American home-trade cheese differs from the American cheddar. Of the English cheeses, the best known are the English Cheddar, Cheshire and Stilton; others worthy of mention are Leicestershire, Lancashire, Derbyshire, single and double Gloucester, Wensleydale and Wiltshire.

Stilton.-The manufacture of Stilton cheese is confined almost entirely to Leicestershire, England. Its manufacture is quite different from that of the common type of English and American cheeses, and in many respects more nearly resembles many of the Continental varieties, and is almost exclusively confined to private dairies. It is made from sweet milk, and more commonly separate curds are made from both night's and morning's milk, the two being brought together when ready for the hoops. Stilton cheese is also made from a single eurd, and in this case the night's milk is held till morning and mixed with the morning's milk. It is commonly supposed that Stilton cheese is made from milk which has been reinforced with more or less cream, but this is not the case at the present time; in fact, Stilton cheese of good quality can be made from 
milk not particularly rich in fat. In making Stilton cheese the fresh milk is put into a circular tin vat of sufficient size to hold the milk of the whole dairy. The milk is allowed to cool to about $85^{\circ}$ $\mathrm{F}$., and enough rennet is.added to cause a firm coagulation in an hour or an hour and a quarter. While the milk is coagulating the strainers are arranged in

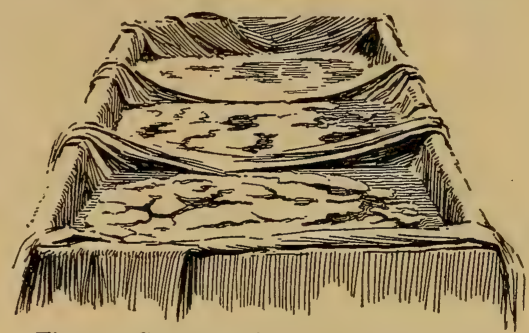

Fig. 46 Straining-cloths containing curd. (Redrawn from Jour. Royal Agr. Soc., 3rd Series, vol. x.)

sinks, as follows: The sinks must be large enough to hold all the milk that is made at one time. Many makers prefer earthenware, though on account of the less cost, sinks of heavy tin are frequently used. They are about six inches deep, two to two and a half feet wide, and of any convenient length, and are simply fitted with outlets and plugs. For straining the curds stout linen cloths are used. These are a yard square and are arranged in the sinks as shown in Fig. 46, and are supported by sticks laid across the sink in such a way that the ends of each cloth may be gathered up without disturbing those next to it. When the milk is firmly coagulated, so that the curd is somewhat harder than for ordinary cheddar cheese making, the plugs are put into the sink and the curd dipped into the strainer with the curd ladle or scoop shown in Fig. 47. This is done with a peculiar deft motion 
in such a way that the scoop takes up a thin slab of curd, breaking it as little as possible. The curd is carefully laid in the strainer, each strainer receiv-

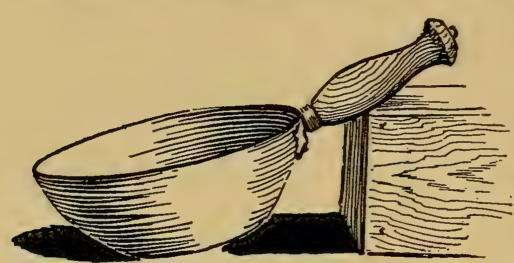

Fig. 47. Stilton curd scoop. ing a slice in turn until all the contents of the vat are collected in the strainers. The strainers when full should contain about three to four gallons. After dipping, the whey begins to separate and is allowed to stand until the curds are well covered, when the plugs are withdrawn and the whey allowed to run off. The ends of the strainers are then gathered up, brought closely about the curd, three corners being held in one hand and the whole tightened with the fourth, as shown in Fig. 48; care being taken in doing this that the curd is not broken. The pressure of the cloth causes the whey to separate more freely, and at intervals of an hour the cloths are tightened about the curd until eight or ten

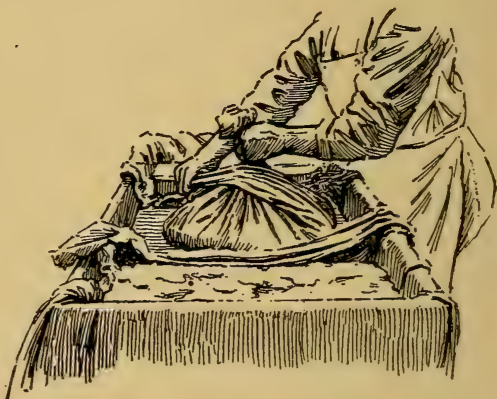

Fig. 48. Method of tightening strainer cloths. (Redrawn from Jour. Royal Agr. Soc., 3rd Series, vol. x.)

hours have passed, when the curd should be firm and solid, and the whey entirely separated. The 
cloths are now removed from the curd and the curd is cut into pieces about four inches square, and spread in the bottom of the sink and allowed to remain till morning or until it has taken on a good degree of acidity, so that it is distinctly sour to the taste. If the room has been held at a temperature between $60^{\circ}$ and $65^{\circ} \mathrm{F}$., this will occur when the night's curd is 36 and the morning's curd 24 hours old. The two curds are then coarsely broken up with the fingers, mixed together, salted (about one ounce to three and a half pounds of curd), and put in the hoop shown in Fig. 49. The

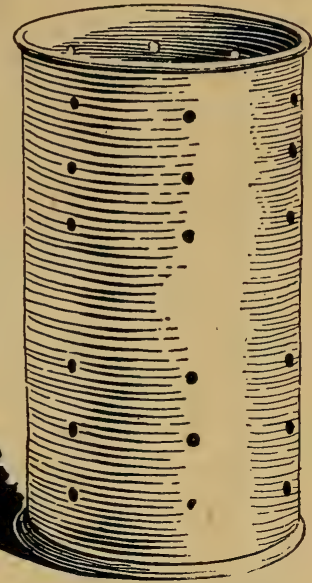

Fig. 49. Hoop for Stilton cheese. hoops are made of tin, 7 inches in diameter and 15 inches high, open at both ends and perforated with numerous holes. The empty hoop is set upon a thin piece of board 9 inches square, covered with a piece of muslin. The curd is put in the hoop loosely, care being taken that the larger pieces fall in the center, and the smaller ones go to the outside. The hoops when full are set aside to drain. Each day the curd is turned in the hoop, a fresh board and piece of cloth being used each time. After six to eight days the curd next the hoop begins to take on a fermentation, which gives it a moist appearance 
and an aromatic odor. The curd also shrinks slightly so that the hoop slips from it easily. The cheeses are then ready for the coating process. The hoops are removed entirely and the cheese taken into another room, where the temperature is maintained at from $55^{\circ}$ to $60^{\circ} \mathrm{F}$., and the air is kept nearly saturated with moisture. The cheeses are scraped with a dull knife, the cracks being filled up with the material scraped from the more prominent places. After the scraping, a bandage is pinned firmly to the cheese. The scraping is continued from day to day for two or three days, clean bandages being put on each time until the coat begins to appear. This is seen in the formation of a white mold, and also in the appearance of dry patches upon the bandage. The bandages are now removed from the cheese, and it remains in the coating-room for about two weeks, being turned every day, and resting on a board covered with cloth. When the coat has fully formed, the surface of the cheese is a light drab, of a wrinkled appearance, and is then ready for the curing-room. The curing-room is kept at the same or a little higher temperature than the coating-room, and the atmosphere may be somewhat drier, though care must be taken that it is not too dry. Curing goes on slowly for two or three months, after which the cheese may be removed to a cool cellar and will continue to improve for some time longer. During the euring process the mold begins to grow in the interior of the cheese, and when fully ripe the cheese should be evenly mottled 
and veined with blue mold through its whole substance. A well-made and well-cured Stilton cheese has a rough, wrinkled, drab exterior. The cheese

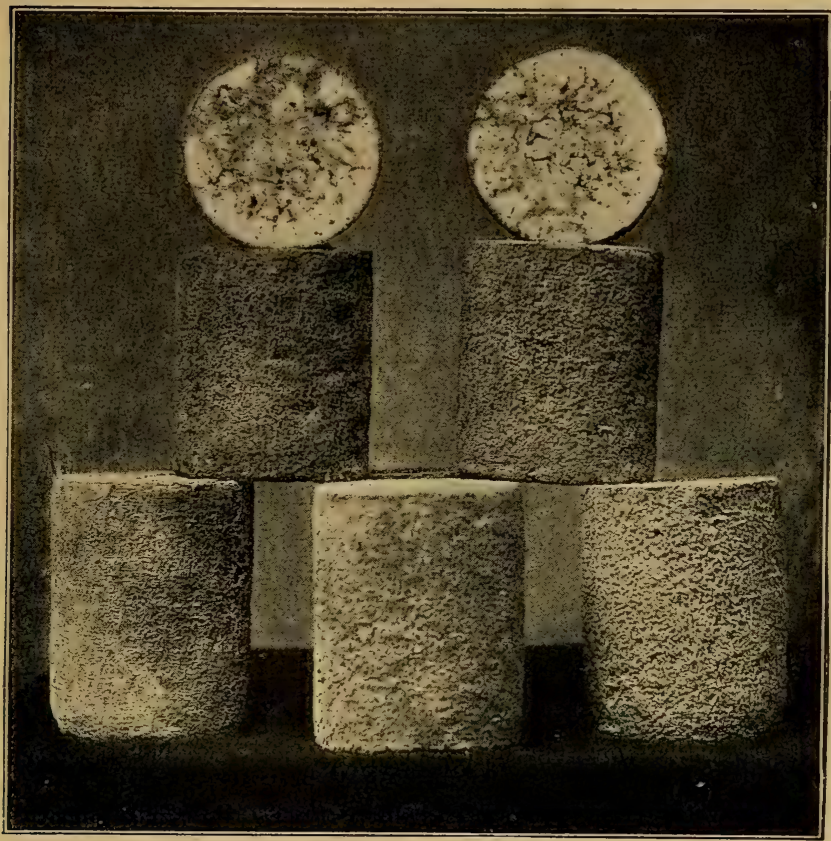

Fig. 50. Stilton cheese, from "Stilton Cheese Making," by J. Marshall Dugdale, Jour. Royal Agr. Soc., 3rd Series, vol. x., through the courtesy of the Secretary of the Royal Agricultural Society of England:

itseif is of a soft, uniform texture, pale-colored, and evenly streaked with mold. The flavor is mild and largely characteristic of the mold. The appearance of the cheese is well shown in the cut, Fig. 50. 
Cheshire.-Cheshire is a cheese of the ordinary type, soft in texture and rather high in flavor. It takes its name from the county in England where it is most largely made, and is one of the most important of English cheeses. Cheshire cheese is made from whole milk, night's and morning's milk mixed together and of some degree of ripeness. In many dairies the night's milk is skimmed in the morning before the morning's milk is added to it, and the cream so obtained reserved to be added to the cream of the following day at the same time that the cream removed the day before is added to the milk from which the cream is taken. This is done in order to secure a certain degree of ripeness. The milk is set at about $85^{\circ} \mathrm{F}$., with enough rennet to cause a rather firm coagulation in about an hour. It is then eut with a perpendicular knife lengthwise of the vat, and allowed to stand ten minutes or until a considerable amount of whey is separated, and is then cut crosswise and a second time lengthwise. It is not ordinarily eut with the horizontal knife at all. After cutting, it is carefully stirred with the hand for about an hour, during the latter part of which time heat is applied to bring the contents of the vat back again to $85^{\circ} \mathrm{F}$. When the eurd is firm enough so that a piece thrown 15 to 18 inches in the air and caught in the hand does not break it is allowed to settle, usually for about half an hour, but before any acid develops in the whey the curd is carefully pushed to one end of the vat and the whey drawn off. 
The curd then mats and is cut and piled much as in the Cheddar system, except that the pieces are cut smaller and the cutting is renewed each time the curd is turned, and the piling is done in such a way that the curd does not flatten out. The acidity and moisture of the curd is regulated during this cutting and piling process. If the curd is too wet, it is turned and piled frequently. If it is too acid and dry it is allowed to lie longer and not turned so often. When some acid is developed, so that the curd takes on a glistening appearance and begins to grow slightly fibrous, it is finely ground in a peg mill, salted and put in the hoops with strainer cloth about it but not under pressure. At night it is turned and returned to the hoops. The next morning it is again turned and then put in the press with very slight pressure. Each succeeding day the turning is continued and the pressure slightly increased until it has been pressed for five days in all. It is then taken from the press and allowed to stand one day in the hoops without pressure, after which it is taken out and a bandage pasted on with flour paste. It is allowed to stand three days in the press-room, and then put in the curing-room at a temperature not above $65^{\circ}$, and cured for three or four weeks. The cheese should all be of the same height, and to bring this about hoops of varying diameter are used so that the variation in milk from day to day will make no difference in the height of the cheese. If a cheese is too high after the first or second day 
in the press it is put in a hoop of larger diameter and vice versa When the cheese is put in the curing-room, the growth of a white mold on the ends is encouraged by laying on the upper end of the cheese a plate or round piece of slate. The cheeses are kept on straw in the curing-room, and when ready for market the ends of the cheese, except where the plate has lain, are cleaned and polished, and the marks of the straw show in the white mold in the center. Cheshire cheese is usually colored. When of good quality it is of a soft and somewhat granular texture, dissolving readily on the tongue, and with a pronounced and rather sharp cheese flavor.

Lancashire.-Lancashire cheese is very similar to Cheshire, though it is made somewhat softer and the flavor is more pronounced. No heat is used to aid in the separation of the whey, and ordinarily, when ready for the press, the curd is divided into two portions, one of which is mixed with the curd retained from the preceding day and pressed, and the other kept to be mixed with the curd made on the following day.

Derbyshire and Leicestershire.-These cheeses are made by modifications of the Cheddar process. They are intended to produce a somewhat softer cheese to ripen in a shorter time. Both are pressed in flat shapes, not over six inches in height and about sixteen inches in diameter. The Derbyshire is white, the Leicestershire highly colored. Both should be covered with dark mold when ready for the market. They bear the same relation to English Cheddar 
cheese that the soft varieties of of home-trade cheese do to American export Cheddars.

Wensleydale.-Wensleydale cheese is made in certain districts in Yorkshire, England. It occupies an intermediate position between the Stilton on the one hand and the ordinary hard cheese on the other. In texture and flavor, and in the characteristic veins of blue mold it quite closely resembles the Stilton, but it is made after a process somewhat resembling the ordinary Derby or Leicestershire or American home-trade processes, and is pressed in a bandage in an ordinary press. It is cured at a temperature of about $60^{\circ} \mathrm{F}$., care being taken that the growth of the mold is facilitated even to the extent of burrowing the cheese with skewers if the mold does not grow with sufficient rapidity.

Gorgonzola.-Gorgonzola is an Italian blue-molded cheese closely resembling Stilton in texture, though it is usually of inferior flavor. Considerable quantities of Gorgonzola are imported into this country, but their quality is not at all uniform and the process of manufacture, resembling that given for Stilton in the main, is not systematically carried out by the peasants in the north of Italy, where it is made.

Emmenthaler, Gruyere, Swiss or Schweitzer.-The cheese made in the mountains of Switzerland has a history reaching back to the seventeenth century,* and many of the old customs are still used; but, as

*Monrad, Cheese making in Switzerland. Winnetka, Ml., 1896.

S 
might be expected, the various localities have developed many varieties of this general type, in the same way that the different forms of cheddar and allied cheese have come to differ from one another. It is generally considered that the cheese known as Emmenthaler is typical of the whole group of Swiss cheeses. The Emmenthaler cheese is made in a large copper kettle instead of a vat, and ordinarily the curd made in one vessel is pressed as a single cheese. After the curd has been coagulated with rennet, it is broken up in various ways into small pieces as nearly uniform in size as possible, and then heated, with careful stirring and attention, up to $135^{\circ}$ or $140^{\circ} \mathrm{F}$. After heating, the curd is allowed to sink to the bottom of the vessel in a solid mass, and while in this condition the bandage is slipped around it and the whole mass of curd conveyed to the hoops, where it is pressed. In the subsequent curing the curd is usually salted from the outside of the cheese as it is curing, and during the curing process certain fermentations go on which produce large holes in the cheese. These holes in perfect cheese should be uniform in size and at equal distances from one another. The casein itself breaks down into a cheese of solid, uniform texture and characteristic flavor. It has been asserted that the characteristic flavors of the Swiss cheese are due to the character of the Alpine pastures upon which the cows feed, but it is altogether likely that the curing fermentations have as much or more to do with developing these flavors, Edam.-The round Dutch cheeses, colored red, 
that are common in all markets take their name from Edam, a small town in North Holland, though the cheeses made in the whole of North Holland are practically of this variety. The cheeses are made almost wholly in private dairies, though there are a few factories in which the milk of several farmers is pooled together. The cheeses are made from partly skimmed milk; ordinarily the milk of the evening is put at once into the cheese making tub, and in the morning the cream that has risen is removed from it for making butter. The fresh morning's milk is added and the whole set immediately at a temperature of $85^{\circ} \mathrm{F}$., with enough rennet to coagulate it in about 40 minutes somewhat softer than for ordinary cheese making. When the coagulation is sufficiently advanced the curd is broken with a wire curd breaker, the bars of which are about threefourths of an inch apart. The breaking is done at first gently into large pieces and afterward more vigorously, a constant agitation being kept up by the breaker which results in breaking the curd into finer and finer pieces. After the whey begins to separate a portion is drawn off and heated upon a stove, the agitation meanwhile of the curd and the remaining whey being constantly kept up. When the whey is sufficiently warm so that when added to the whole mass it will bring the contents of the tub up to $85^{\circ} \mathrm{F}$., the heated whey is added and the stirring with the breaker kept up for a full hour, at the end of which time the particles of curd should be about the size of kernels of wheat, and firm and hard, but still entirely 
sweet. The curd is then allowed to settle on the bottom of the tub. When it is sufficiently matted so that it will hang together it is carefully turned over and doubled up at one side of the tub, which is slightly elevated for the purpose. The whey is then dipped off and the curd made as firm as possible in the tub by pressure with a piece of board and an iron weight. When all the whey has been removed that will run off readily, the curd is broken up with the hands and packed in the

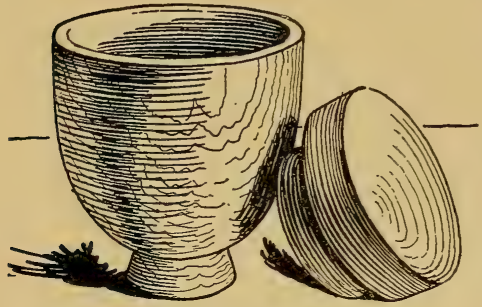

Fig. 51. Mold for Edam cheese. molds shown in Fig. 51.

It is made as firm as possible in the mold by pressure with the hand until it will maintain its shape. It is then removed from the mold, neatly wrapped in cotton cloths and replaced. The covers are put on and the molds put in the press for three hours. When removed from the press the cheese should be round or nearly round in shape. They are put on cup-shaped supports and salted by thoroughly rubbing the outside with salt. They are then set aside, covered with salt, turned and rubbed frequently for three or four days. (Sometimes the salt is added by immersion in saturated brine.) They are kept in the salt as long as any whey is drawn out and are then put on shelves in the curing-rooms still in the cup-shaped support to help maintain their form. The curing-rooms are kept at ordinary temperatures. In 
many places they are the cow stables that are vacant for the summer. The temperature will seldom rise higher than $65^{\circ} \mathrm{F}$. After about three or four weeks they became hard and firm and covered with a white mold. They are then taken down, thoroughly washed

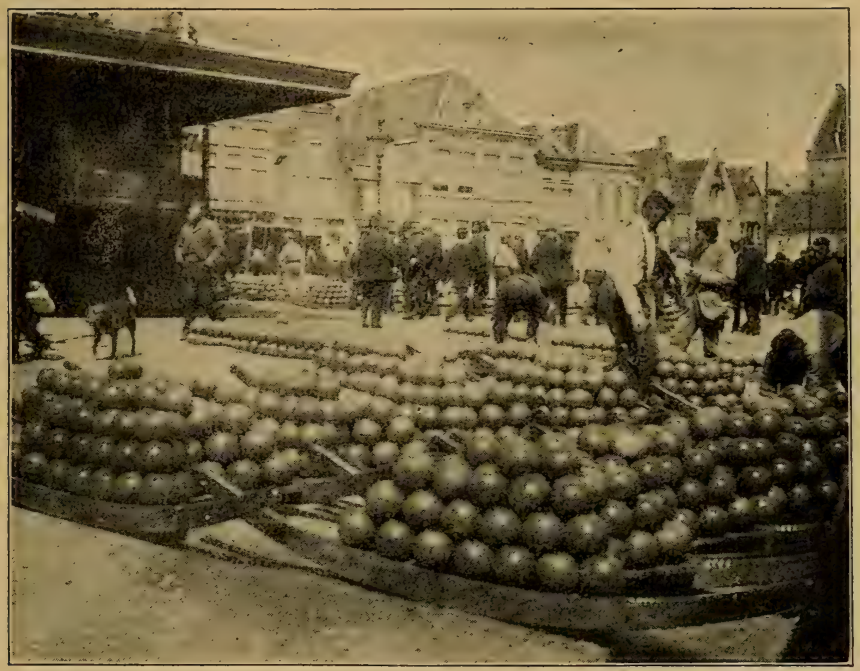

Fig. 52. The Market Square, Alkmaar, Holland. From a photograph by the author, May 21, 1897.

in whey, all the mold scrubbed off, dried and greased with linseed oil, when they are ready for the market. The merchants purchase them in large numbers at the weekly markets in the different villages. A typical market scene is shown in the cut (Fig. 52). When taken to the warehouses they are colored red, wrapped in tin foil and packed for transportation. The euring 
process is not completed at the time the cheese is marketed. An Edam cheese at three or four weeks old is of a tough, elastic texture and scarce any flavor. Six or eight months or more are required to break down the casein and develop the flavor as it is found in the perfect Edam cheese, and this ordinarily takes place while the cheeses are in the warehouse or in course of exportation.

Gouda.-Gouda cheeses are made in South Holland by a process very similar to the manufacture of Edam. The whey is not so completely separated, and the resulting cheese is therefore softer. It is pressed in molds of a flat, oval shape and about ten or twelve pounds weight, and is cured faster than Edam, so that at six weeks to two months old it is a cheese similar in flavor and texture to a well made Cheddar, although somewhat drier.

Roquefort.-This is a soft or semi-soft cheese, made in France. In some respects it is the most famous of all varieties of cheese. It is sometimes made from goats' as well as cows' milk, and it has peculiar characteristics imparted by specific fermentations that are brought. about in the curing process. The curing is done in caves in limestone rock, where the air is uniform in temperature, and in order to bring about the desired fermentations the germs are added to the curd in the process of manufacture. These germs are often cultivated upon bread or similar substance, and this, crumbled up, is mingled with the curd in order to insure the proper fermentation in the cheese. Roquefort cheese, when well made and 
cured, is of a rather soft texture, and the whole mass is permeated with the molds, imparting a characteristic flavor to the cheese.

Brie.-Another soft French cheese. The milk is put into small, circular vessels and the rennet added. The curd is allowed to remain until it has become sufficiently firm to be removed from the whey in one piece. It is then carefully taken up and put in such a position that the whey may drain from it. It is turned frequently and carefully until sufficient whey has drained away so that the cheeses will maintain their form. They are then lightly salted and put away to cure. During the curing process molds develop on the outside, but the fermentations that go on upon the inside of the cheese result in the breaking down of the casein into a creamy mass of a strong, piquant flavor. The molds upon the outside give to the cheese a strong odor of decomposition.

In very many cases, Brie eneese is put upon the market in a very much more immature form, so that it has a firm, though somewhat soft texture. If allowed to become fully ripe, though, it breaks down into a semi-liquid mass similar to Camembert.

Camembert.-Camembert cheese is a soft cheese of French manufacture 3 to 4 inches in diameter and $1 / 2$ to $3 / 4$ inches thick. When fully ripe, the cheese is coated with a heavy growth of red or reddish brown mold, and the interior breaks down into a soft, plastic semi-liquid mass, of pungent odor and piquant flavor. In the manufacture of Camem- 
bert cheese the rennet is added at a rather low temperature and in small quantity, so that eight to ten hours are required before the curd is firm enough for the further treatment. In some cases a culture of the mold from an old cheese is added to the milk before the rennet, in order to facilitate the growth of the mold during the ripening process. When the milk is coagulated into rather a firm curd, the curd and whey is ladled into the mold shown in the cut, Fig. 53, which is placed upon a straw mat arranged on a table so that the whey may drain off. In ladling the curd into the mold care is taken that each ladleful is deposited with as little breakage of the curd as possible, and particularly that the mold, when full, is covered at the top with but a single slice of the curd. The curds are allowed to remain

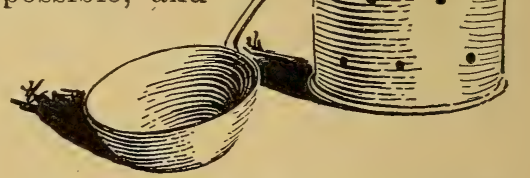

Fig. 53. Ladle and mold for Camembert cheese. in the molds until enough whey has escaped so that the cheeses may be turned without breaking the curd. They are then carefully turned and put upon fresh mats, at intervals of twelve hours, when in the course of three or four days the cheese will be sufficiently firm so that the molds may be removed. After the molds are removed, turning is continued until the mold begins to appear on the surface. The cheeses are then put in a damp curing-room, at a 
low temperature $-55^{\circ}$ to $60^{\circ}$ - for the remainder of the ripening process, which reguires from four to six weeks.

D'Isigny.-D'Isigny cheese is similar to Camembert and Brie, and intermediate in size between them; in fact, D'Isigny so closely resembles Brie that they are often indistinguishable, and, as a matter of fact, D'Isigny is little more than a trade name for a smaller form of Brie.

Pont L'Evêque.-Pont L'Evêque is a soft French cheese of rather firmer consistency than Camembert. The milk is set at the usual temperature, and when the curd is firm enough to handle, it is gently cut into rather large cubes, which are carefully placed upon a straw mat. As the whey begins to separate and run off and the curd becomes firmer, the ends of the mat are brought together in such a way that a slight pressure is brought upon the curd. This is continued until the curd is firm enough so that it can he placed in tin molds like that shown in the eut, Fig. 54. The molds are filled and placed upon a straw mat. The molds are turned once or twice a day until the cheese is firm enough so that the mold may be removed. Mold begins to appear upon the cheese, which is kept at a temperature a little above

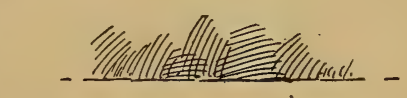
$60^{\circ}$. When the cheeses are well molded they are removed to a damp room of a temperature from $55^{\circ}$ to $58^{\circ}$. When the curing process is com- 
plete, the cheeses are of a soft consistency similar to that of an unripe Brie, but with a characteristic, well ripened flavor fully developed.

Port $d u$ Salut is one of the firmest French cheeses, and when well made is of a texture similar to the best type of American home-trade cheese, with a pleasant, nutty flavor. The milk is set at a rather high temperature and enough rennet added to bring firm coagulation in thirty minutes. The curd is then cut and heated to $104^{\circ}$ and allowed

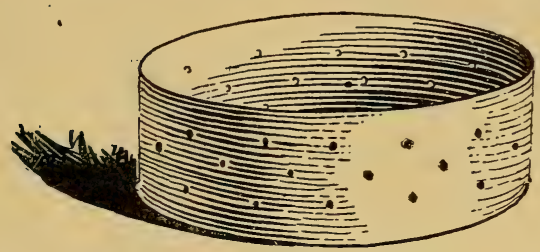

Fig. 55. Hoop for Port du Salut cheese. to remain in the whey until it takes on a peculiar rubber-like consistency. It is then put in the hoop shown in the cut, Fig. 55, which is lined with a strainer cloth and subjected to slight pressure. When taken from the press it is slowly ripened at a low temperature.

Parmesan. - A very hard Italian cheese made from milk with very little fat, and from which a large part of the water has been expelled. In curing it takes on a rather sharp flavor, and naturally it can be kept in almost any climate for almost any length of time. It is so hard that it is ordinarily grated before being used, and is almost wholly used to add piquancy to soups, sauces, and the like. 


\section{CHAPTER XVI}

\section{ICE CREAM}

Relation to dairy practice. -The nutritive value of ice cream, together with its extreme palatableness, makes it one of the leading foods of today. Thus the immense demand has forced it, in a large measure, from the realm of the housewife to the commercial channels of trade. As it is, strictly speaking, a product of milk, the business of making it has fallen in many cases upon the dairyman. The indications for the future seem to demand a knowledge of the principles and practice underlying the manufacture of ice cream for every one interested in dairy products. Although some believe ice cream to be a direct descendant of the sherbet of the Orient, and hence of ancient origin, its development has been rather slow until recently. Catharine de Medici is said to have had frozen ices about the middle of the sixteenth century. Charles II was served with frozen milk at a banquet in the seventeenth century. In the eighteenth century, ice cream was made to some extent in England, Germany, France and the United States. The first advertisement of ice cream appeared in a New York newspaper called "The Post Boy," June 8, 1786, and read as follows: "Ladies and Gentlemen may be 
supplied with Ice Cream every day at the City Tavern, by their humble servant, Joseph Crowe."

The wholesale ice cream business was originated in the nineteenth century by Jacob Fussel, but it reached its maximum development in the twentieth century. The word "ice cream," which corresponds to the European word "ices," is rather a generic term, and may be used to apply to any one of a large class of frozen delicacies.

Classification.-Ice creams in America are divided into two large classes.

I. Philadelphia, or plain cream, which consists of pure cream, sugar and flavoring matter.

II. Neapolitan, which has, in addition to the cream and sugar, beaten eggs, making it, in reality, a frozen custard.

Janet McKenzie Hill makes the following classification:

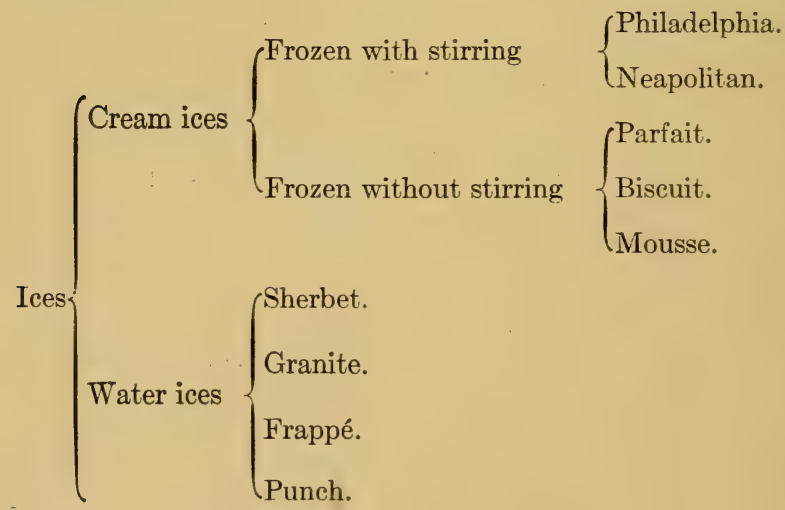


Another classification made by Mortensen* is as follows:

I. Plain ice creams.

II. Nut ice creams.

III. Fruit ice creams.

IV. Bisque ice creams.

V. Parfaits.

VI. Mousses.

VII. Puddings.

VIII. Aufaits.
IX. Lactos.

$\mathrm{X}$. Ices.

1. Sherbets.

2. Milk sherbets.

3. Frappés.

4. Punches.

5. Soufflés.

The definitions and descriptions of the above classification are given as follows by Mortensen:

Plain ice cream is a frozen product made from cream and sugar, with or without a natural flavoring.

Nut ice cream is a frozen product made from cream and sugar and non-rancid nuts.

Fruit ice cream is a frozen product made from cream, sugar, and sound, clean, mature fruits.

Bisque ice cream is a frozen product made from cream, sugar, and bread products, marshmallows or other confections, with or without natural flavoring.

Parfait is a frozen product made from cream, sugar and egg yolks, with or without nuts or fruits and other natural flavoring.

Mousse is a frozen whipped cream to which sugar and natural flavoring have been added.

Pudding is a product from cream or milk, with sugar, eggs, nuts and fruits, highly flavored.

Aufait is a brick cream, consisting of layers of one or more kinds of cream, with solid layers of frozen fruits.

\footnotetext{
*Iowa State College, Bulletin 123, page 357.
} 
Lacto is a product manufactured from skimmed or whole sour milk, eggs and sugar, with or without natural flavoring.

Ices are frozen products made from water, or sweet skimmed or whole milk, and sugar, with or without eggs, fruit juices, or other natural flavoring.

A sherbert is an ice made from water, sugar, eggalbumen, and natural flavoring, and frozen to the consistency of ice cream.

Milk sherbert is an ice made from sweet skimmed or whole milk, with egg-albumen, sugar, and natural flavoring, frozen to the consistency of ice cream.

Frappé is an ice consisting of water, sugar, and natural flavoring, and frozen to a soft, semi-frozen consistency.

Punch is a sherbet flavored with liquors, or highly flavored with fruit juices and spice.

Souffle is an ice made from water, eggs, sugar and flavoring material. It differs from sherbets mainly in that it contains the whole egg.

As a product can be no better than the materials which enter into its composition, it behooves the ice cream maker to use care and discretion in the selection of ingredients.

Quality of cream.-Cream being the main constituent, its quality has a marked effect upon the finished product. The fat content of the cream influences the flavor in proportion to the percentage. This is recognized as such an important factor that the federal and some state governments are requiring that all ice cream offered for sale contain a certain percentage of fat. 
It is a self-evident fact that the flavor of the raw cream is one of the most important factors in ice cream making. It should be free from all "cowy" or weedy flavors, and from all old, rancid, or metallic flavors, which indicate unclean dairying. Although the ice cream maker is not always directly responsible for the cream before it reaches him, he is responsible for the marketable product; so a close supervision of the entire production of the cream is worth while. Granted that the cream is clean and sweet, it is known that the viscosity increases with age, particularly if the cream is held at a low temperature. This viscosity permits an increase in yield because of a greater swell in freezing, while the extreme cold apparently hardens the fat and gives to the frozen product a better body.

Whether cream should be heated or pasteurized depends on the individual. The various chefs and connoisseurs differ. Professor Mortensen recommends pasteurization of eream at $140^{\circ}$ to $145^{\circ}$, and holding it in a retarder for thirty minutes; then cooling it rapidly and holding it at a temperature near freezing for about forty-eight hours. Some people dislike ice cream made from uncooked cream, claiming that it has a raw flavor and less body.

The use of homogenized milk or cream, that is, one which has been made homogeneous throughout by subjecting it, in an apparatus adapted for that purpose, to a pressure of from 3,000 to 5,000 pounds per square inch, is said to enable the ice cream maker to use a cream containing from 16 to 17 per cent fat, 
in which the resulting product will have a body and texture equal to that resulting from the use of a 20 to 25 per cent cream.

Sugar.-The kind and amount of sugar is left usually to the taste of the maker or the demand of the trade. Ordinarily granulated sugar is used, though some prefer XXXX or some of the finer grades. Other manufacturers believe that brown sugar gives a delicate flavor which is desirable.

Whether the sugar should be added in the form of syrup is another question which is open. Experience has shown that where syrup is used the resulting product is perhaps of finer grain or body, but that the time for freezing is longer, and it is also a little more difficult to keep the cream frozen and in good condition. Not all makers may find that this is so.

Flavors.-The flavoring matter may be obtained in various ways; the many kinds of vanilla extract, both natural and synthetic, on the market give the manufacturer a wide range for choice. Some believe that the best results are obtained by extracting the vanilla from the beans themselves with alcohol. The other flavors, such as caramel, chocolate, maple, and the various fruits, may be procured already prepared from some commercial concern, or perhaps as good, if not better quality, may be made by the maker himself. Vanilla has that peculiar quality which enables it to combine with other flavors in such a way that the result is particularly pleasing. Thus, in caramel and chocolate creams, some vanilla is usually used. Most recipes advise the addition of the fruits when the 
cream is partially frozen and not at the beginning of the process.

Fillers.-As before stated, in the Neapolitan or French types of cream eggs are used; the increase in body or smoothness being very slight, the value of the eggs is more nutritive than otherwise. All kinds of starches are used to give more substance to a cream with a low percentage of fat. Rennet in various forms is used for the same purpose. Gelatin has been used in commercial creams for some time. It not only adds body but, if carefully used, prevents the crystallizing of moisture upon standing, and keeps the cream smooth. Gum tragacanth, a vegetable gum, is used for the same purpose as gelatin; its tenacity is so great that only a very small amount is needed, and for this and other reasons it is gaining favor. Whether or not these fillers or binders should be used is questionable. Suffice it to say that they are, strictly speaking, adulterations, and so, from the standpoint of purity in food products, should be discountenanced.

Freezing and packing.-Authorities differ on the amount and kind of salt that should be added to the ice in freezing. In most cook-books coarse salt in the proportion of one of salt to three of ice is recommended, but some recent experiments* show that, if the ice is very fine, the proportion may be 1:12 or even $1: 20$. It is important that the greater proportion of salt be placed midway or on top, as salt at the bottom of the freezer is largely wasted.

*Vermont Agricultural Experiment Station, Bulletin No. 155. 
The finer the salt, the more rapidly it dissolves and the more quickly cold is produced, thus hastening the freezing process. However, it is believed by many that it is better that the cream should be cooled slowly at first, at least until after it reaches the whipping point. The addition of water to the salt and ice, thus making a brine, will increase the freezing to a considerable extent. The freezer should also be turned slowly at first, for rapid agitation before passing below the churning point causes small lumps of butter to form. After this point, the cream should be beaten or whipped by rapid revolution of the dasher. If the speed is slow throughout, there will be little swell and a coarser texture. The entire process should take from fifteen to twenty-five minutes, depending upon the temperature of the cream in the beginning; the cooler the cream, the less time will be required.

Transferring and packing.-If only a single batch is made, it is most common to clean off the dasher and repack the ice around the freezer. However, if it is necessary to transfer the cream to another receptacle, it should be done while fresh and in a rather soft condition. If allowed to harden, and then re-dished, there will be a considerable loss of volume. In packing or repacking, a coarser grade of salt may be used, as the amount of cold needed is only sufficient to maintain the frozen condition.

Freezers.-The essential parts of an ice cream freezer are, first, the vessel in which the cream or other material to be frozen is contained. This is fitted with an agitator, with which to stir the cream during 
the process, and which, in turn, is set in a larger container, which holds the ice or other refrigerating material.

Ice cream freezers of several types or classes are now in general use, the chief distinction being as to whether the cream is frozen in successive batches, or continuously, or whether brine or melting ice is used as the refrigerant. The various types may be classified as follows: Vertical batch ice, vertical batch brine, horizontal batch brine, and horizontal continuous brine.

The first type is the one in common use, and for small quantities is practically the only type used. They are furnished in any size up to 10 gallons, and may be run by either hand or power. A 10 -gallon freezer will require for the first batch from 10 to 20 pounds of ice and from 5 to 6 pounds of salt. Succeeding batches will require less ice because the machine has been cooled, and the freezing process is ready to begin almost from the start.

When artificial refrigeration is available, the brine freezer has the advantage of better control of temperatures, and does away with the inconvenience of handling large amounts of ice. Brine freezers are arranged so that, instead of crushed ice surrounding the container, refrigerated brine is circulated around it. Batch machines of this sort may be placed either vertically or horizontally, and they are often so arranged that the finished ice cream may be drawn away from the bottom or side without removing or stopping the dash.

The last type of horizontal freezer is the latest evolution of freezing machinery, and differs from the 
other types in that the cream flows in at one end of the machine and the finished ice cream out at the other, refrigeration being secured by moving disks through the center, through which the brine is circulated. This machine is open, and the process can be watched and temperatures taken as it progresses. These machines are quite often operated as "batch" machines; that is to say, the cream is allowed to flow in until the freezer is full, or nearly so, and the finished product drawn out, not continuously, but from time to time.

Recipes.-With the wide variety of materials that are used in making the various kinds of ice cream, it is, of course, impossible to make anything like a complete list of recipes. The following are given as typical of the various classes, and are intended to convey something of the proper proportions of cream, sugar and flavoring materials. Vanilla ice cream may be taken as representative of the types of plain ice cream, and three standard formulæ are given below.

A.* This is, doubtless, the most common of ice creams. To make 10 gallons, one should use about 50 pounds ( 6 gallons) of aged 18 per cent cream (or $5 \frac{1}{2}$ gallons of cream and $1 / 2$ gallon of condensed milk), about one-sixth that quantity (8 pounds) of sugar, 3 to 4 ounces of vanilla extract; and 3 to 4 ounces of gelatin, or 1 quart of "gumstock," if a binder seems called for.

B. To make a single gallon of ice cream, one should

*Vermont Experiment Station, Bulletin No. 155. 
use 2 quarts of 22 per cent cream ( 4 pounds), seventenths pounds ( 11 ounces) of sugar, $1 \frac{1}{2}$ tablespoonfuls of vanilla extract.

C. $*$ To make 10 gallons of finished ice cream, use 5 gallons of 25 per cent eream, 8 pounds of sugar, and 4 ounces of vanilla.

There is little or nothing to be gained by the use of gelatin in ice cream made at home for immediate consumption. The sugar should be thoroughly dissolved in the cream before the freezing process is started, although, in many conditions, the time and amount of agitation given before the cream freezes is sufficient to dissolve and to mix in the added sugar. Experience only can indicate the safety of this point to the maker. The flavoring may be added at any time before the mass starts to freeze.

Vanilla ice cream is especially accommodating in that, though of itself it is one of the most popular flavors, its flavor is yet so delicate that it easily gives way to other and stronger ones, like coffee and chocolate; so that, if but a single quart or gallon of coffee ice cream is ordered, it is not an infrequent practice in the trade to mix a small quantity of the desired flavor with a sufficient quantity of vanilla ice cream, and thus to accommodate the consumer and relieve the dealer of certain embarassments. Or if, say, vanilla, coffee, and strawberry ice creams are desired, the stock for the entire three kinds is made up as one batch, then used first as vanilla, to the extent desired,

*Iowa State College, Bulletin No. 123, page 357. 
then as coffee, and then as strawberry, one after the other, all from one and the same vanilla mix and without washing the machine, and usually to the entire satisfaction of all concerned.

The following may be taken as representative formulæ for the various kinds of ice cream, according to Mortensen's elassification:

Chocolate Ice Cream-

5 gallons cream.

10 pounds sugar.

$1 \frac{1}{2}$ pounds bitter chocolate.

4 ounces vanilla extract.

Maple Ice Cream-

5 gallons 25 per cent cream.

6 pounds cane sugar.

2 pounds maple sugar.

2 ounces caramel.

4 ounces vanilla extract.

Caramel Ice Cream-

5 gallons 25 per cent cream.

8 pounds sugar.

12 ounces caramel.

4 ounces vanilla extract.

Coffee Ice Cream-

5 gallons 25 per cent cream.

8 pounds cane sugar.

Extract from 1 pound coffee.

Mint Ice Cream-

5 gallons 25 per cent cream.

8 pounds cane sugar.

1 pint concentrated Creme de Menthe syrup.

Few drops green coloring.

Walnut Ice Cream-

5 gallons 25 per cent cream.

8 pounds cane sugar.
4 ounces vanilla extract.

4 pounds walnut meats.

Strawberry Ice Cream-

5 gallons 25 per cent cream.

8 pounds sugar.

1 quart crushed strawberries.

Lemon Ice Cream-

5 gallons 25 per cent cream.

10 pounds sugar.

2 pints lemon juice.

1 pint orange juice.

Orange Ice Cream-

5 gallons 25 per cent cream.

10 pounds sugar.

2 quarts orange juice.

$\frac{1}{2}$ pint lemon juice.

Macaroon Ice Cream-

5 gallons 25 per cent cream.

8 pounds sugar.

4 ounces vanilla extract.

5 pounds ground macaroons.

Walnut Parfait-

4 gallons 30 per cent cream:

Yolks of 10 dozen eggs.

14 pounds sugar.

4 ounces vanilla extract.

4 pounds ground walnut meats. 
By substituting for the vanilla extract and nut meats, the same proportion of fruits as are used for fruit ice cream, fruit parfaits, such as strawberry, raspberry and cherry parfaits, and others, may be prepared.

\section{Coffee Parfait-}

4 gallons 30 per cent cream.

Yolks of 10 dozen eggs.

14 pounds sugar.

Extract from 1 pound coffee.

Tutti Frutti-

4 gallons 30 per cent cream.

Yolks of 10 dozen eggs.

14 pounds cane sugar.

4 ounces vanilla extract.

3 pounds candied cherries.

3 pounds candied assorted fruit.

Cranberry Mousse-

2 gallons 30 per cent cream.

4 pounds cane sugar.

1 quart cranberry juice.

$\frac{1}{4}$ pint lemon juice.

Nesselrode Pudding-

3 gallons 30 per cent cream.

10 dozen eggs.

10 pounds cane sugar.

4 ounces vanilla extract.
6 pounds candied cherries and assorted fruits.

4 pounds raisins.

4 pounds walnut meats.

4 pounds filbert meats.

Lemon Sherbet-

6 gallons water. Whites of 2 dozen eggs.

24 pounds sugar.

6 pints lemon juice.

$1 \frac{1}{2}$ pints orange juice.

Pineapple Milk Sherbet-

6 gallons milk.

20 pounds sugar.

Whites of 2 dozen eggs.

1 gallon pineapple pulp.

1 quart lemon juice.

Pineapple Souffé-

$1 \frac{1}{2}$ gallons water.

4 dozen eggs.

12 pounds sugar.

1 gallon grated pineapple.

1 quart lemon juice.

Scoring.-The quality of ice cream may be judged by a score card in the same way that other dairy products are scored. A score card proposed by the Iowa Experiment Station is as follows: 
1. Flavor.......................... 45

2. Texture......................... 25

3. Richness....................... 15

4. Appearance.................... 10

5. Color ........................ 5

Total. .................... 100

The points in the score card may be described, explained, and criticised as follows:

I. Flavor.-The flavor, to be perfect, must be clean and creamy, and combined with flavoring material in such a way as to blend with the cream in a full and delicious flavor. Defects in flavor are various, and are usually due to some one or more of the following causes or conditions:

(1) Defects due to the use of flavoring materials which will not blend with the other ingredients.

(2) Defects due to cream used:

Sour cream flavor.

Old cream flavor.

Bitter cream flavor.

Metallic cream flavor.

Oily cream flavor.

Weedy cream flavor.

Barn flavor

Unclean flavor.

Burned, overheated or scorched flavor.

(3) Defects in flavor due to filler used:

Condensed milk flavor.

Starch flavor.

Gum flavor.

Gelatine flavor. 
(4) Defects in flavor due to other ingredients:

Too sweet.

Lack of sweetness.

Coarse flavor due to flavoring material.

Stale fruit flavor.

Rancid nut flavor.

Mouldy nut flavor.

II. Texture.-Cream of perfect texture must be firmly frozen and be smooth and velvety. The more common defects in texture may be described as:

Icy.-Due to the presence of lumps of ice in the cream. It is most noticeable toward the bottom of the container, and may be due to improper packing or to holding too long ice cream which was manufactured without filler.

Coarse.-This defect may be due to the use of too thin cream, or to packing while too soft.

Sticky.-Due to too large use of such fillers as gelatine, sweetened condensed milk, glucose, etc.

Buttery.-This defect is due to the use of cream which has been partially churned before freezing, or to cream which enters the freezer at too high a temperature, in which case it is churned or partially churned while freezing. It may also be due to operating the freezer at a too high speed, or to some defect in the construction of the freezer.

Too soft.-Due to improper packing after freezing.

When judging ice cream containing nuts, fruits, or other solid material, due allowance should be made for the presence of such ingredients.

III. Richness.-Ice cream containing the amount 
of butter-fat required by the state pure food law should be considered perfect in richness. The richness is determined by making chemical analysis for fat.

IV. Appearance.-Ice cream scoring perfect in appearance should be clean, and neatly put up, and in a clean container.

Defects.-Cream of unclean appearance; lack of parchment circle over ice cream; dirty container; rusty container; dirty ice cream tub; old string tags attached to handle of tub.

When judging brick ice creams, special attention should be given to the uniformity of the layers, to the neat folding of the parchment wrapper, and to cleanliness and general appearance of the package.

V. Color.-Ice cream of perfect color is such as contains only the natural color imparted to it by the flavoring material used; or, if color is added, it should harmonize with the particular flavoring used.

Defects in Color.-Too high color; unnatural color, such as colors different from the color of the natural flavoring material used.

Individual molds, if colored, should be as nearly as possible the same color as the object they represent. 


\section{CHAPTER XVII}

\section{OTHER AND BY-PRODUCTS OF THE DAIRY}

THE by-products of the dairy are skimmed milk, buttermilk and whey, and a variety of products that may be manufactured from them. The utilization of these by-products to the best advantage is an important part of the economy of dairy manufacture.

Skimmed milk, buttermilk and whey.-By far the larger part of the dairy by-products must of necessity be utilized as food for animals, either because of the cost of transportation or for lack of facility in marketing many of the rather perishable products that can be made from them. All of these products make a valuable food for animals, of course in proportion to the amount of the normal constituents of the milk which they contain. Whey is less valuable than skimmed milk or buttermilk, because it has lost the greater part of its casein as well as fat, but it still is of sufficient value to render its utilization of importance. Naturally, we expect that young animals (calves and pigs) will thrive the best upon these products, though skimmed milk has frequently been fed to cows with good results. All three are, however, so bulky that some more concentrated food 
should be fed in connection with them, if they are used to the best advantage. When economically fed to young pigs and calves, skimmed milk and buttermilk may be made to return about fifteen cents per hundred weight, and whey about one-third less. These products are of value for food in proportion as the milk sugar has not been changed to lactic acid. They may be fed in unlimited quantities without ill results upon the health of the animal, except that occasionally when the milk is very sour, or when fermentations other than lactic have set in, derangements of the digestive organs, diarrhœa, etc., sometimes occur. It is, therefore, advisable that all of these products should be fed in as fresh a condition as possible, and it has been found in many instances that the custom of sterilizing or partially sterilizing the skimmed milk or whey at the factory, by injecting a jet of steam into it until the whole is heated up to about $180^{\circ} \mathrm{F}$., is practical, and is followed by beneficial results.

Condensed milk.-In 1856 a patent was granted to Gail Borden, Jr., on a process for "concentrating sweet milk by evaporation in vacuo, having no sugar or other foreign matter mixed with it." From small beginnings the business has grown to enormous proportions, and is still largely in the hands of the descendants of the original patentee.

There are two classes of condensed milk, namely, sweetened and unsweetened.

Sweetened condensed milk. - Sweetened condensed milk was the first condensed milk to successfully reach 
the market in hermetically sealed cans. It is made from cows' fresh milk. The milk is first heated to near the boiling point, then sucrose (cane sugar) is added to the extent of about 16 pounds of sugar to 100 pounds of fresh milk. The milk and sugar solution is condensed

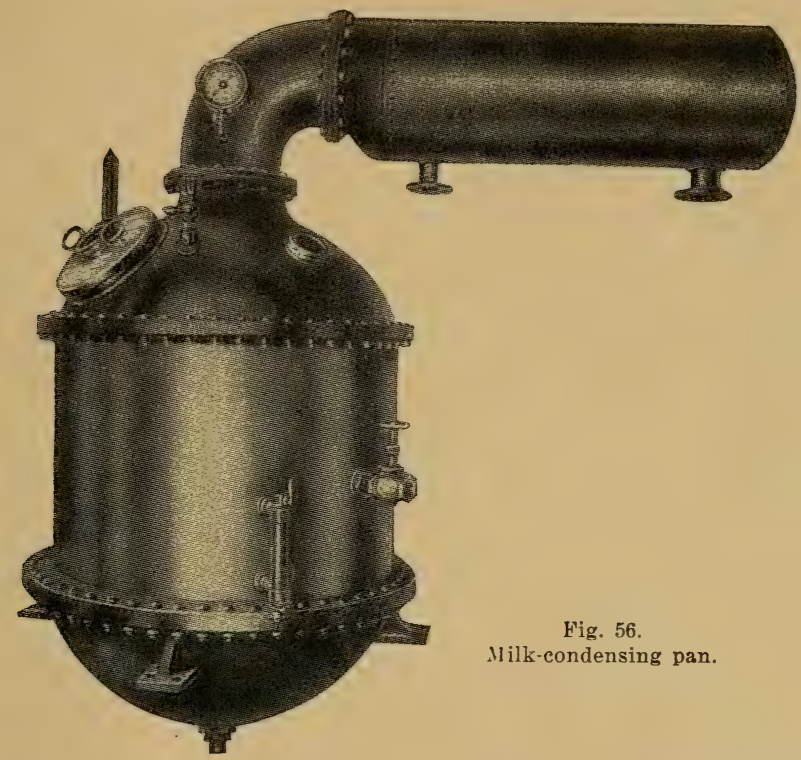

in vacuo at a temperature of $130^{\circ} \mathrm{F}$. to $150^{\circ} \mathrm{F}$. The ratio of concentration is about $2 \frac{1}{2}: 1\left(2 \frac{1}{2}\right.$ parts of fresh milk are condensed to 1 part of condensed milk). The finished product contains about 40 per cent sucrose, it is of semi-liquid consistency and has a specific gravity of about 1.29. It is sold partly in hermetically sealed tin cans varying in capacity from 
8 to 20 ounces, and retailing at from 5 to 20 cents per can; and partly in barrels holding from 300 to 600 pounds of condensed milk. The barrel goods are sold to bakeries and confectioners. They are generally made from partly or wholly skimmed milk.

Sweetened condensed milk is not sterile, but is preserved by the sucrose it contains; it will keep for a considerable length of time, but is best when fresh.

Unsweetened condensed milk.-There are three kinds of unsweetened condensed milk, namely, evaporated milk, plain condensed bulk milk, and concentrated milk.

Evaporated milk. - In the manufacture of this product, cows' fresh milk is heated to near the boiling point, then condensed in vacuo at $130^{\circ} \mathrm{F}$. to $150^{\circ}$. F. The ratio of concentration is about $2^{1} / 4: 1$ ( $2 \frac{1}{4}$ parts of fresh milk are condensed to 1 part of evaporated milk). The evaporated milk is then filled and sealed in tin cans varying in size from 8 ounces to 1 gallon. The hermetically sealed cans are sterilized at temperatures ranging from $226^{\circ} \mathrm{F}$. to $240^{\circ} \mathrm{F}$. for from 30 to 60 minutes. From the sterilizer the cans are transferred to the shaker, where they are subjected to violent agitation, to completely break up the coagulum that may have formed during sterilization. The finished product has the consistency of cream of medium richness, and has a specific gravity of about 1.065. It sells at from 5 to 50 cents per can.

Evaporated milk is sterile, it keeps indefinitely, but is best when fresh.

Plain condensed bulk milk. -This kind of condensed milk is made from whole milk, partly skimmed, or 
skimmed milk. The fresh milk is heated to about $160^{\circ} \mathrm{F}$. and condensed in vacuo at $130^{\circ} \mathrm{F}$. to $150^{\circ} \mathrm{F}$. The ratio of concentration is 3 to $4: 1$ ( 3 to 4 parts of fresh milk are condensed to one part of condensed milk). Before it leaves the vacuum pan, it is superheated by live steam to swell or thicken it. It is sold in 10-gallon milk cans. It has the consistency of very thick cream, and has a specific gravity of about 1.09. This product is largely sold to ice cream manufacturers, and limited quantities of it are retailed in milk bottles to the direct consumer. It sells at from 25 to 75 cents per gallon.

Plain condensed bulk milk is not sterile. Its keeping quality is similar to that of a good grade of pasteurized milk.

Concentrated milk.-Concentrated milk is made from fresh milk. Before condensing, the cream is removed, and the skim milk is condensed at $140^{\circ} \mathrm{F}$. by blowing hot air through the milk. When the concentration is completed, the cream is returned to the condensed product. The ratio of concentration is about 3:1 (about 3 parts of milk are condensed to 1 part of concentrated milk). This product is used for direct consumption. It differs from plain condensed bulk milk only in that it has not been exposed to so high temperatures during the process.

Concentrated milk is not sterile. It keeps about as long as a good grade of pasteurized milk. Its manufacture is very limited.

The chief essential in the successful manufacture of all condensed milks is a good quality of fresh milk. 
Milk that is abnormal in its properties when drawn, badly contaminated milk, and milk that has not been properly and promptly cooled on the farm cannot be safely used in the condensery. Such milk either does not withstand the trials of the process, or it succumbs to the many and unfavorable conditions to which it is subjected on its long journey from the factory to the pantry of the consumer. Its original defects magnify with age and follow it to its destination, resulting usually in heavy loss to the manufacturer.

Milk powders.-Several processes for completely removing the water from milk have been invented and are in more or less successful operation. The resulting product is in the form of a creamy white powder which upon agitation readily unites with water, thus restoring the milk. Skimmed milk thus treated is much used for the manufacture of custards and other bakers' products; but the powder from whole milk soon becomes rancid and is very little used.

Dried casein, paper sizing. - The casein of milk in a dry form is useful in certain manufacturing processes, and has been found to be particularly valuable for the preparation of sizing for paper, the preparation of paint and various other uses. The manufacture of dry casein for this purpose has come to be an important means of utilizing skimmed milk in many ereameries, and its preparation is comparatively simple. The skimmed milk is collected in a vat and the curd precipitated with a mineral acid. A mixture of acetic and sulphuric acids is commonly used, and from $1 / 2$ to 1 per cent 
is sufficient. When the curd has formed, which will be as soon as the acid is thoroughly mixed with the milk, the whey is drawn off and the surd washed two or three times with warm water. It is then taken out and pressed as dry as possible, after which it is ground rather fine in a peg mill and then dried in an oven till perfectly dry. The amount usually realized from the skimmed milk in this way is not more than may be realized when it is skilfully fed to animals.

Milk sugar.-Milk contains between 4 and 5 per cent of milk sugar, and the manufacture of this sugar has come to be an important industry. In the manufacture, whey is preferably used, or if skimmed milk the casein is first coagulated and removed. The water is then removed by evaporation until the crystals of sugar are formed. Various methods are used to rid the sugar of albumin and other materials contained in the whey. Formerly this was a somewhat difficult operation, and added considerably to the expense of the manufacture, but recently improvements have been made in this respect, so that the cost of the manufacture of the sugar is much lessened. The growth of the industry has been very rapid, resulting in a much lower price for the product and a very much increased consumption. Formerly, almost the only use of milk sugar was medicinal. Now, because of its digestibility, it forms an important ingredient of many of the so-called infants' and invalids' foods. It is usually prepared in the form of a white crystalline powder, only mildly sweet to the taste. 
Dutch rlieese (rottage cheese, schmiertase, pot cheese, etc.).-A toothsome and nutritious article of food is made from sour skimmed milk or huttermilk hr allowing the casein to coagulate hy the action of lactic acid already formed, and then expelling the water by the aid of heat. A considerable number of products locally distinct, and differing in the degree of dryness of the casein, are made in this way, the general process of manufacture being to take sour buttermilk, or skimmed milk which has coagulated, heating gently to from $85^{\circ}$ to $125^{\circ} \mathrm{F}$, according to circumstances, draining off the whey through a cloth strainer, and then reducing the texture of the resulting curd by kneading with the hands or a pestle; salt is added, and the product is improved by the addition of a small amount of cream or butter, and occasionally by the use of some of the more common spices, as nutmeg, caraway, etc. It is commonly made only for domestic consumption, but in most cities and villages, especially during the summer months, there is a considerable demand for fresh cheese of this sort, and its manufacture is often a source of revenue to factories suitably located. It is usually sold and eaten in a fresh state, but it may be subjected to certain curing processes, which quite materially change its character, and which vary widely in different localities.

Whey cheese (primost and myseost).-These are products manufactured from whey in some of the north European countries and among the Scandinavians in our own. They are really forms of evaporated whey 
and, according to Monrad, ${ }^{*}$ are made somewhat as follows: The whey, not too sour, is boiled in a suitable vessel under a slow fire, care being taken that it is not scorched or burnt; when the albuminous matters are coagulated they are removed to facilitate evaporation, and the evaporation is carried on until the whole mass assumes a syrupy condition: the albuminous matters are then returned to the condensed whey, the whole is removed from the fire and mixed rapidly until in the form of a thick mush; some cream is then added and the material pressed in brick shaped moulds, and after a day or two is ready for market. It is practically unknown except in those districts where the Scandinavian population is large.

Cheese food. - Within the last few years an enterprising cheese-maker, Mr. J. J. Angus, of Wisconsin, has perfected a system of manufacturing what he calls a complete cheese food. It is simply a product containing all the constituents of the milk in a condensed form. An ordinary cheddar cheese is first made and cured; the whey is evaporated to a syrupy consistency, and the cheese from a corresponding amount of milk is ground down to a pasty consistency and mixed with the evaporated whey. The resulting mass is pressed into cakes of convenient size, and under ordinary conditions will keep a long time. The cheese food is a soft, homogeneous substance of a mildly cheese-like, sweetish flavor.

*d B C of Cheese-making. Winnetka. IIl, 1889, 
Koumiss is a beverage made from milk that has undergone alcoholic fermentation. It resembles buttermilk in taste, but has a frothy appearance and the casein of the milk is coagulated into a fine curd. It was originally made from mares' milk by various wandering tribes in Russia and Asia. It has been found to possess certain dietic properties, and is now prepared from cows' milk by various formulas. According to Fleischman, one of the best formulas is as follows:

One hundred pounds of separator skim milk is mixed with 42 pounds of water, 1.75 pounds of granulated sugar, .75 pounds of milk-sugar and $5 \frac{1}{2}$ to 6 ounces of good yeast, and is allowed- to stand for 32 hours at a temperature of $100^{\circ} \mathrm{F}$. During this time the mixture is stirred about six times at equal intervals. It is then decanted into patent-stoppered bottles, the stoppers secured and the bottles put into a cellar at a temperature of $55^{\circ} \mathrm{F}$. It should be used within six days.

Kephir is a similar beverage to Koumiss. In its preparation the fermentation is brought about by certain bodies, the so-called Kephir grains, of which very little is known. Kephir is but little known in this country.

Wheyn.-Quite recently there has been patented in this country by Alexander Bernstein, of Berlin, Germany, a nourishing, mildly stimulating drink with the above name. It is, according to the specifications of the letters patent, a sour, sterilized whey, from which the albuminous matter and fat have been re- 
moved. It is put up without further treatment, or it may be flavored with hops or other material, which may also be carbonated, or subjected to a mild alcoholic fermentation 


\section{CHAPTER XVIII}

\section{BUTTER AND CHEESE FACTORIES}

Location of creameries. - In the location of a creamery no one thing is more important than to secure a well drained site, and yet this is in many cases evidently the last thing thought of. Creameries are located in any out of the way corner or bit of waste land that happens to be at hand. The drainage is no less an important matter to be considered in selecting a creamery site than in selecting a house site. The foundation of cleanliness in a creamery begins with the sewer, and unless the waste materials can be completely and quickly drawn away the labor of keeping the whole premises clean is very much enhanced.

Other things being equal, then, elevated ground should be selected as a proper site for a creamery, and if the advantage of the elevation can be made use of in the arrangement of the working rooms, so much the better. Another matter that may prove of considerable value in many cases is a regard for the natural or artificial shelter that may be given the creamery building. A difference of six or eight degrees in the work-room temperature may be easily secured by having a due regard to the advantages of 
shade from groves or the channels of natural air drainage. On the other hand, it is not well to locate the building on a too bleak or exposed site, and yet the great majority of creameries are either ex-

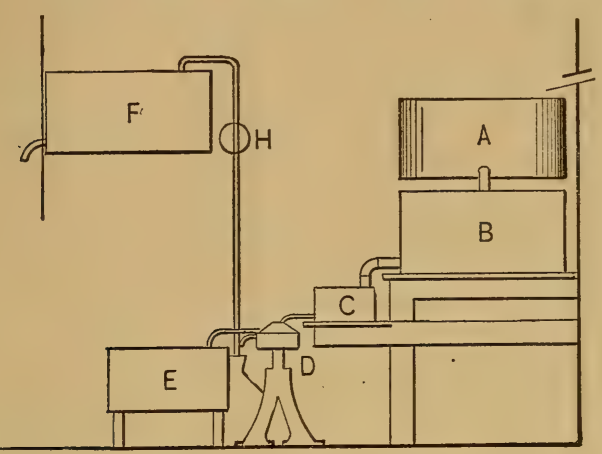

Fig. 57. Diagram of creamery arranged upon the "gravity" plan. posed to the full rays of the August sun or to the bleak winds of winter.

Arrangement of buildin $g .-\mathrm{T}$ w o general principles govern the arrangement of creamery buildings. In the one, the milk is taken in at such an elevation that it may flow by gravity from the weighing ean to the receiving vat, thence to the tempering vat, thence to the separator, and finally to the skimmed milk and cream vats. In the other, the milk is taken in on a level with the work-room floor, and is elevated by pumps. Both plans have their advantages and disadvantages. The main advantage of what may be called the "gravity" system is, that the milk flows by its own weight during the whole course of manufacture, and no pumps, troublesome to keep clean, are necessary. As an offset to this advantage, it entails a considerable amount of extra labor in ascending and descending the neces- 
sary stairs or steps. The pumping system, on the other hand, is economical of labor, but intro-

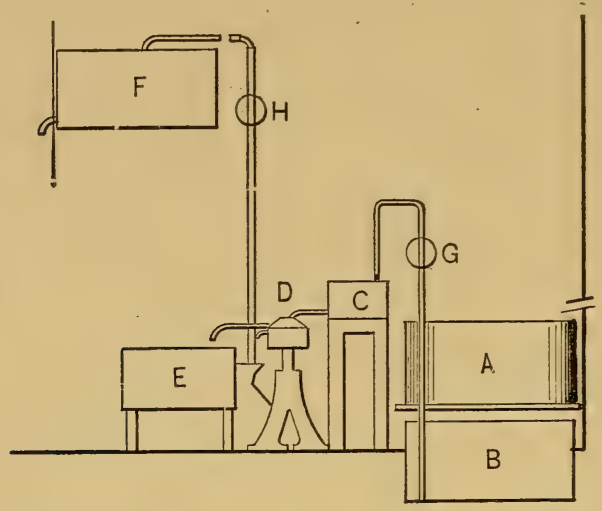

Fig. 58. Diagram of creamery arranged upon the pumping" plan. duces one or more pumps, through which the milk must be passed, and which are always, even when of the simplest pattern, more or less difficult to keep clean, and exceedingly liable to be neglected. In

the outline diagrams is a creamery arranged according to either system. By reference to the ground plan (Fig. 59, page 329), it may be seen that in either case the amount of floor space required is the same. In Fig. 57 is shown the elevation under the "gravity" arrangement, with the exception that the skimmed milk vat is shown elevated. Here the milk is taken in at $A$, passes to $B$ the receiving vat, thence to $\mathrm{C}$ the tempering vat, thence to $\mathrm{D}$ the separator, thence to $\mathrm{E}$ the cream vat, and through the pump $\mathrm{H}$ to $\mathrm{F}$ the skimmed milk vat. In the pumping system, Fig. 58, the same letters indicate corresponding parts. It will be seen here that the milk is pumped through $\mathrm{G}$ from $\mathrm{B}$ to $\mathrm{C}$. Referring 
again, then, to the floor plan, if the arrangement be according to the pumping system, the operator, stand-

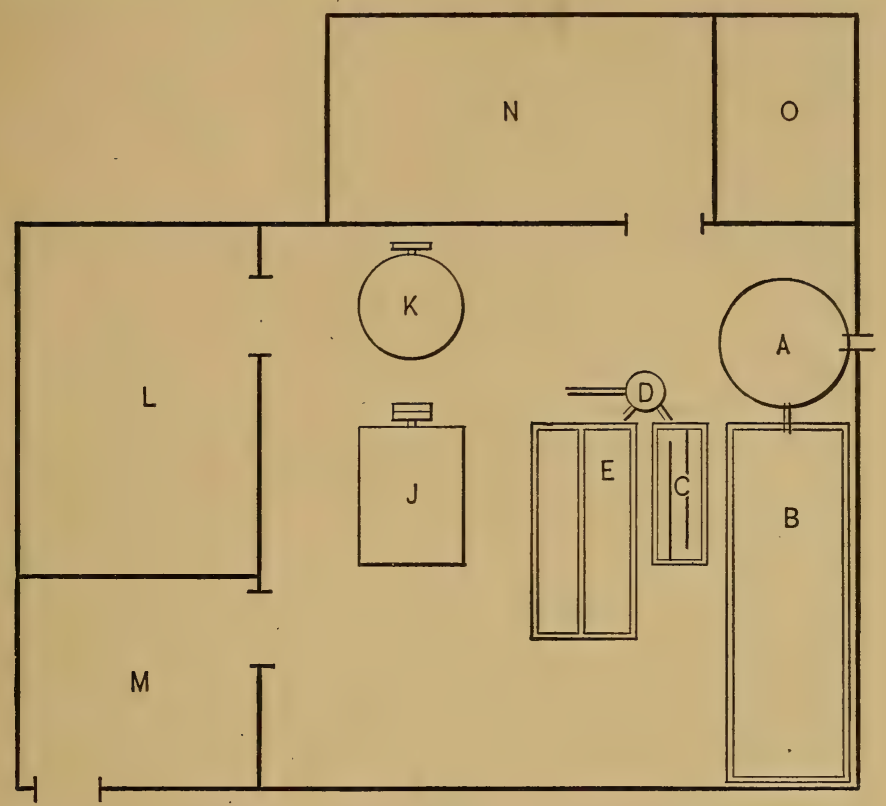

Fig. 50 Ground plan of ereamery in Figs. 39 and $40 . A$, weighing can ; $B$, receiving vat; $C$, tempering vat; $D$, separator; $\dot{E}$, cream vat; $J$, churn: $K$, butter worker; $L$, cold storage room; $\boldsymbol{M}$, office ; $N$, boiler room ; $O$, fuel.

ing near his scales and receiving vat $\mathrm{A}$, is but a step from the separator $\mathrm{D}$ and the boiler $\mathrm{N}$, and ean easily give attention to all parts of his factory. Whereas, if the creamery is arranged upon the "gravity" plan, he must be constantly ascending and descending a series of steps between $A$ and D. It would seem, therefore, that when the factory is to be a 
large one, requiring the services of more than one man, that the "gravity" system is preferable, because of doing away with the objectionable pumps. Whereas, if the factory is to be a small one, and one man is to do all the work, the amount of labor saved by the more compact pumping system will more than overbalance the objection arising from pumps. In a factory of the size indicated, handling from three to five thousand pounds of milk per day, a floor space of about $18 \times 24$ feet will be required. In larger factories, it will be found of advantage, in controlling the temperature, to introduce partitions, so that the receiving and separating room may be separated from the churning and working room, and if much butter is to be made up into prints, a third, colder room, for printing the butter, will be found of great advantage during the warm part of the year. Here, too, the question of labor again comes into play. When the creamery is so small that all the work is done by one man, labor is economized by having all the operations conducted in one room, but where two or three, or even more men are employed, it will be found of advantage to have separate rooms for the different operations.

Construction.-The building should be laid upon solid foundation walls rather than upon piers. Besides adding decidedly to the appearance of the building, it aids materially in keeping the building warm in winter and cool in summer. In regard to the floors, cement floors are the most expensive 
and the most durable, and if well laid and composed of the best materials, are entirely satisfactory. But wooden floors, tightly laid upon a well-drained foundation, are fairly durable, and when out of repair may be easily replaced at comparatively small cost. Whatever the floors, they must be thoroughly sewered. The floor should slant rapidly to the gutter, so that all water will quickly run off throngh a thoroughly trapped iron pipe until the outside of the building is reached, after which well-glazed sewer pipe may be used. An abundant supply of pure water is indispensable. This, under ordinary conditions, will be obtained from a well. A bored or driven well is less liable to contamination than an open dug well, but in any event the site of the well should be chosen with due regard to possible sources of contamination. If the water is very abundant and at a temperature below $48^{\circ}$, a supply of ice is not indispensable, except for cold storage purposes, but it will be found in most cases to be of great advantage. In any event, the means of readily securing and maintaining in the cream a temperature of $50^{\circ} \mathrm{F}$. or below is indispensable. Water and steam pipes should be carried to all parts of the building where their presence may be of advantage for cleaning purposes.

The superstructure may be as elaborate or as simple as desired. At the least, it should offer as good protection from the elements as a well-built house does. In fact, the construction need not 
differ from that of ordinary house construction, except that ceiling on the inside take the place of plaster.

The inside finish should be of matched and planed lumber, thoroughly protected with hard oil.

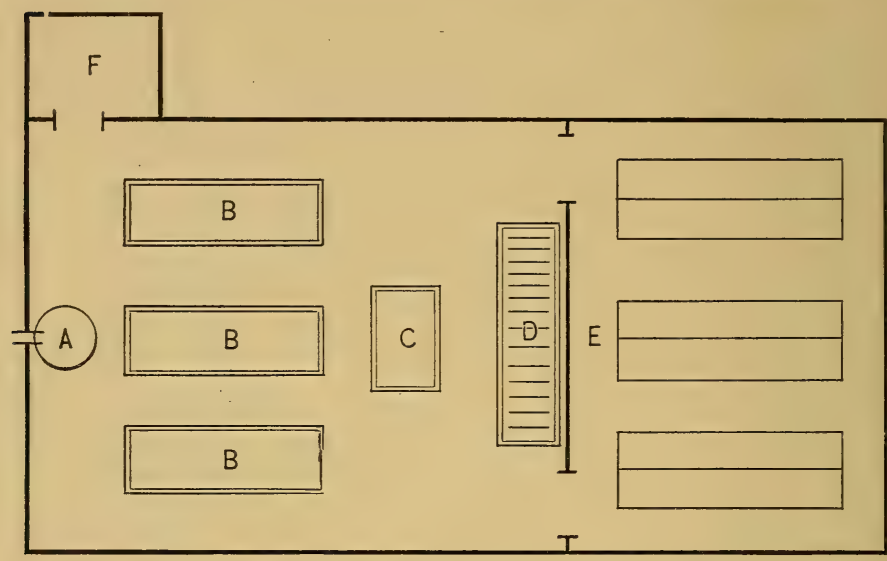

Fig. 60. Plan of cheese factory properly arranged.

It should be needless to say that the outside should be neatly and tastefully painted, yet well-painted creameries are the exception and not the rule.

Cheese factories. - All that was said in regard to the location of creameries applies with equal force to cheese factories. Heretofore, perhaps, even less attention has been paid to the drainage of cheese factories than to creameries, but it is quite as necessary. The arrangement of the cheese factory is considerably simpler than that of the ereamery, and mis- 
takes in arrangement are less liable to be made. The governing principle should be that the milk, in its transformation from milk to cheese, should pass in one direction from the weighing can toward the curing room. The outlines given, Figs. 60 and

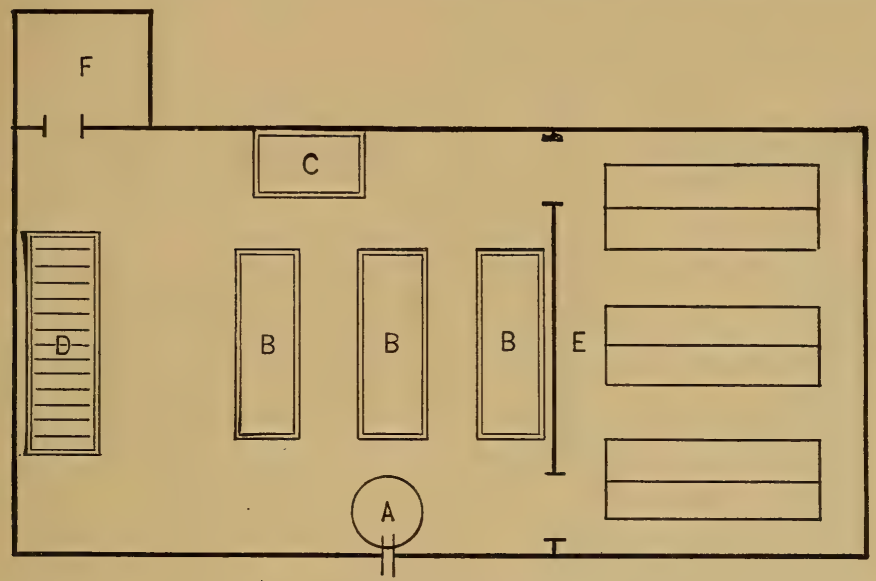

Fig. 61. Plan of cheese factory improperly arranged.

61, show factories properly and improperly arranged. In Fig. 60, the milk taken in at $A$ passes into the vats at $B$, from thence to the curd sink $C$, and the press $\mathrm{D}$, and finally to the curing room $\mathrm{E}$, constantly in one direction and with a minimum of hand labor. Whereas, in Fig. 61. following the same letters, the milk or curd travels from one end of the room to the other and back again. While cheese factories, as a rule, are used only during the warm months of the year, and perhaps for that reason 
may be less warmly built, still it is not sufficient that the superstructure be merely of siding nailed to the joists, leaving the inside bare. While, perhaps, it is not necessary that the outer siding be double, or that building paper be used upon the joists, still the joists should always be ceiled upon the inside and the ceiling neatly finished in oil. The construction of the curing room is the most important part of the cheese factory. Here the construction should be of such a nature that a fairly constant temperature may be maintained. This can hardly be secured unless the building is covered on the outside with two thicknesses of boards, with paper between and tightly ceiled upon the inside. This part of the factory, at least, should always be upon a solid wall foundation. Constructed in this way, if care is taken to ventilate the building thoroughly at night, and to close it tight during the day time, a fairly cool curing room can be secured even during the hottest weather; but the temperature can be much more satisfactorily regulated if the ventilation comes through a sub-earth duct for a considerable distance. This may be arranged by laying a three or four-inch glazed sewer pipe at least four feet deep, from a point on the surface fifteen or twenty rods from the building, and opening into the center of the curing room through the fiour. If the mouth of this sub-earth duct can be placed in such a position that the prevailing winds will blow toward it, a circulation of cool air can more easily and certainly be secured. 
Combined butter and cheese factories. - The connection between the butter and cheese markets is such that it is coming to be of considerable advantage for a factory to be able to make either butter or cheese. In many cases but little additional room or expense is necessary. If in the creamery the receiving vat is of the same construction as an ordinary cheese vat, that is, piped so that hot water or steam can be introduced around it, all that is necessary in order to make cheese is to add a cheese press and the necessary curing room. In a cheese factory the addition of a separator, churn and worker serves to transform it into a creamery. It is true that the presence of the necessary equipment is always a temptation toward the making of skimmed milk cheese during a part of the year, but a due regard for the reputation of the products of the factory will always result in making either full cream cheese or butter alone. Naturally the manufacture of cheese is most advantageous during the summer months, and the manufacture of butter most profitable during the winter months. It is not at all unlikely that dairy manufacture will tend in this direction in the future, instead of certain localities being devoted almost exclusively to butter manufacture and others to cheese manufacture, as in the past.

Farm dairy buildings.-Heretofore the farm dairy work has shared with the other farm industries and the domestic life the room necessary to its prosecution, and this has been and still is a main reason for the general inferiority of farm dairy products. In 
order that the farm dairy be successful, it is necessary that some certain place be set aside rigorously for its prosecution. The dairy cannot be associated with the kitchen, the vegetable cellar, or any other part of the farm buildings except to its own detriment. The room or building devoted to the farm dairy need not be large nor elaborate. It should simply secure a room of convenient size, shut off from any other occupation, well lighted, well ventilated, well drained, and reasonably cool in temperature. It is entirely possible that this room be a part of the house cellar, but if so it should have its own individual exit, and be completely shut off from the cellar at large. In the same way it may be a part of any of the farm out-buildings, or it may be a building by itself. As to whether in a moderate sized dairy, where say twenty to forty cows are kept, the dairy should have a building separate from others or not, depends largely upon the way in which the dairy work is carried on. If the dairy is so large that power is required for the churning, or if the cream is raised by a gravity process, it will undoubtedly be of advantage that the dairy should occupy a separate building; but if the cream is separated by centrifugal process, and power is not required for churning, then the milk may be separated in the barn adjoining the stable, and only the cream carried to the dairy room proper. There are many advantages in this latter plan. In the first place, the labor of carrying the milk to the dairy, and the skimmed milk and buttermilk away, is done 
away with. In the second place, the room required for ripening the cream and churning the butter is very small, even for a dairy of considerable size, and may easily be fitted up in a cool corner of the cellar. The only disadvantage is that steam for cleaning the utensils is not conveniently at hand, but the advantages in most cases will outweigh this single aisadvantage. If this requisite of the farm dairy building or apartment is secured, namely, a room devoted to nothing but butter or cheese manufacture-light, airy and well drained-there is no reason why the very highest quality of products should not be made under such conditions, and there are several reasons why a better product can be made than under the ordinary factory conditions, where the production and care of the milk is in the hands of a large number of persons, over whom the butter-maker or cheese-maker can at best exercise only partial control. 


\section{C'HAPTER XIX}

\section{SIATISTICS AND ECONOMICS OF THE DAIRY INDUSTRY}

THE dairy industry in its development has fully kept pace with other industries and with the population. The total number of milch cows has increased between two and three fold since 1850 , though in 1890 there were slightly fewer cows in proportion to the population than in 1850 . But while the number of cows has not increased faster than the population, their product has materially done so. This is seen in the average yield per cow and in the increase in the production of butter and cheese. In 1850 the average yield per cow was $166^{1 / 2}$ gallons. This had increased in 1890 to 315.4 gallons, or nearly 100 per cent. The total production of butter was nearly four times as much in 1890 as it was in 1850 , and the production of cheese slightly less than $2 \frac{1}{2}$ times as much.

The various details of this development are well shown in the following tables, compiled from the United States Census reports by the Dairy Division of the United States Department of Agriculture :*

\footnotetext{
No. 1)

*U. S. Dept. Agr., Bureau of Animal Industry, Bulletin No. II (Dairy
} (338) 


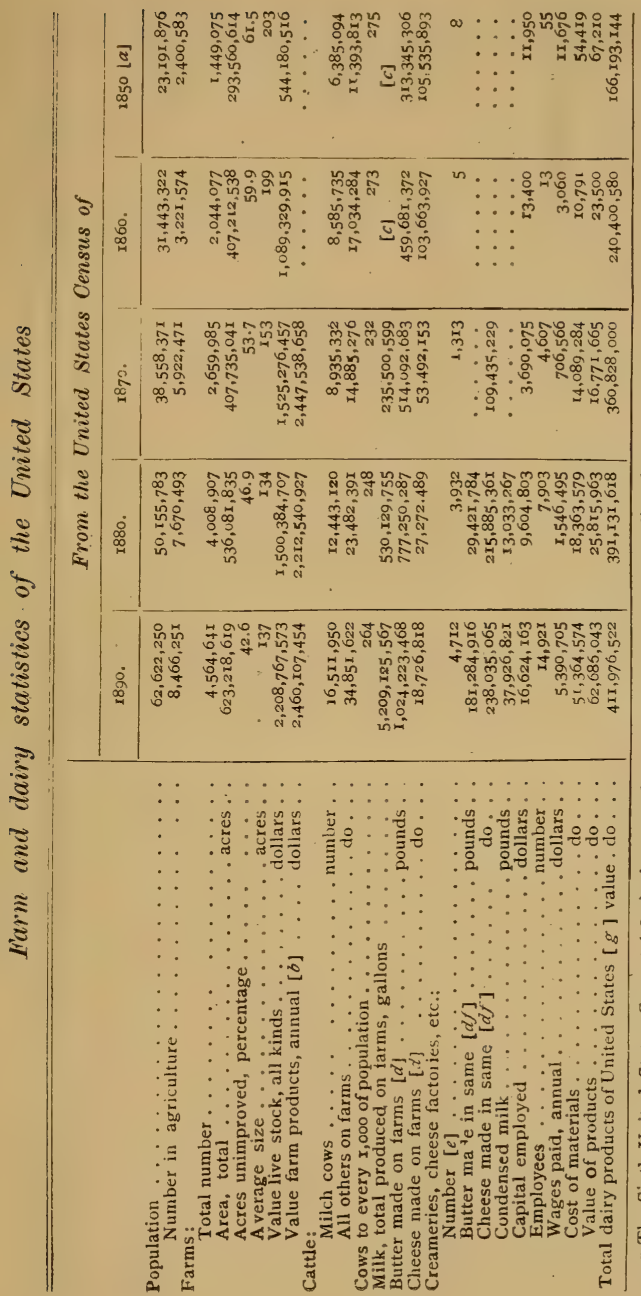

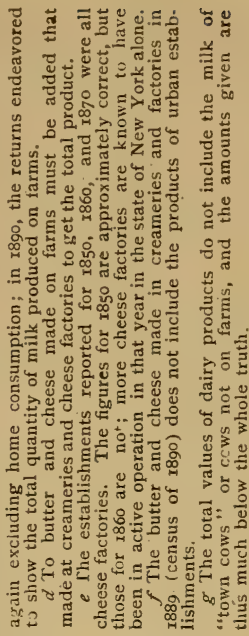

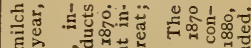
号

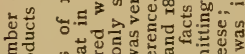
要 y

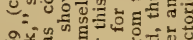

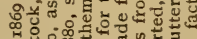
家

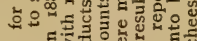

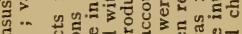
ช。 \& 员 is 可 万人 平揱 क人

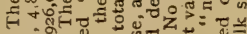

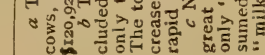


Talue of principal farm products of the United States

\begin{tabular}{|c|c|c|c|c|c|c|}
\hline \multirow[b]{2}{*}{ Products. } & \multicolumn{2}{|r|}{1859.} & \multicolumn{2}{|r|}{1879.} & \multicolumn{2}{|r|}{1889.} \\
\hline & $\begin{array}{c}\text { Per } \\
\text { cent. }\end{array}$ & Total value. & $\begin{array}{l}\text { Per } \\
\text { cent. }\end{array}$ & Total value. & $\begin{array}{c}\text { Per } \\
\text { cent. }\end{array}$ & Total value. \\
\hline $\begin{array}{l}\text { Meats . . } \\
\text { Corn } \\
\text { Hay } \\
\text { Dairy products } \\
\text { Wheat } \\
\text { Cotton } \\
\text { Poultry } \\
\text { Other products }(a)\end{array}$ & $\begin{array}{r}17.9 \\
21.6 \\
9.1 \\
14.4 \\
7.5 \\
12.6 \\
4.5 \\
12.4 \\
\end{array}$ & $\begin{array}{r}\$ 300,000,000 \\
360,680,878 \\
152,671,168 \\
240,400,580 \\
124,635,545 \\
211,516,625 \\
75,000,000 \\
206,639,527\end{array}$ & $\begin{array}{c}22.1 \\
19.2 \\
11.3 \\
10.8 \\
12 \\
7.5 \\
5 \\
12.1 \\
\end{array}$ & $\begin{array}{r}\$ 800,000,000 \\
694,818,304 \\
409,505,783 \\
391,131,618 \\
436,968,463 \\
271,636,121 \\
180,000,000 \\
440,438,353 \\
\end{array}$ & $\begin{array}{c}23.9 \\
15.9 \\
14 \\
11 \\
9.1 \\
8.2 \\
5.3 \\
12.6\end{array}$ & $\begin{array}{r}\$ 900,000,000 \\
597,918,829 \\
526,632,062 \\
411,976,522 \\
342,491,707 \\
307,008,114 \\
200,000,000 \\
472,492,249 \\
\end{array}$ \\
\hline Grand total. . & 100 & $\$ 1,671,544,323$ & 100 & $\$ 3,624,498,642$ & 100 & $\$ 3,758,519,483$ \\
\hline
\end{tabular}

$a$ "Other products" include barley, buckwheat, flax fiber, flaxseed, hemp, hops, Irish potatoes, leaf tobacco, maple sirup, maple sugar, oats, rice, rye, sorghum molasses, sweet potatoes, and wool.

But it is not so much in the amount of dairy product manufactured as in the way the business is done that the dairy industry shows its most remarkable advances. Up to 1850 the whole dairy output was produced, manufactured, and marketed from individual farms. Since then the introduction and wonderful growth of associated dairying, or the factory system, has taken place, and this period has also witnessed the introduction of so many and so varied machines and utensils that the dairy practice of forty or even twenty years ago is entirely revolutionized in the methods of to-day. But while associated dairying has made rapid strides, both in butter and cheese making, it is only in cheese making that the factory system can be said to have at all supplanted private dairying. In 1890 only a little more than 7 per cent of all the cheese produced was made outside of factories; while in the 
same year, of the $1,205,508,384$ pounds of butter made in the country, only $181,284,916$ pounds, or about 15 per cent, was made in factories.

Development of the factory system. - Associated dairying, or the manufacture of the milk of several patrons at one place, under the eye of a single person, was at first limited wholly to cheese making. The system may be said to have been inaugurated by Jesse Williams, in Oneida county, N. Y., when in 1851 he began the manufacture of milk, produced by himself and several sons located on farms near by, into cheese under his immediate supervision. From this beginning the number of cheese factories increased, slowly at first but afterward more rapidly, until in 1870 there were in operation 1,313 cheese factories. Up to this time butter factories were unknown, but within a few years began to be rapidly established, and in 1890 there were of both butter and cheese factories 4,712. Ten states-New York, Wisconsin, Iowa, Ohio, Pennsylvania, Illinois, Vermont, Minnesota, Michigan and Kansas, in the order named-contained nearly 90 per cent of all the factories. Of these there were in New York 1,337, in Wisconsin 966, and in Iowa 500, or nearly 60 per cent of the whole.

When the first butter factories or creameries, as they are more generally called, were established, the milk of the several patrons was drawn to the factory, set in deep cans, usually surrounded by running water, and afterward skimmed and churned. After a time the gathered-cream system was introduced. 
Under this system the cream was raised upon the farm, usually by a cold deep-setting process, and the representative of the creamery, visiting the different farms, skimmed the cream, and left the skimmed milk upon the farm. Later on, during the decade beginning in 1880, the centrifugal separator was introduced, and at the present time by far the larger number of butter factories are operated upon this system. As between the factory system or the private dairy in the manufacture of dairy products, both have their advantages and disadvantages. The advantages of the factory system are so great that practically all of the cheese is made in this way, the small amount made upon farms and in private dairies being almost wholly made for domestic or strictly local consumption. These advantages in the main are the saving of labor and the greater uniformity of product. It requires no more time and but slightly more labor to make six thousand pounds of milk into cheese than six hundred. Few private dairies produce more than the latter amount, so that the combination of ten men in a factory will result in saving the labor of at least five men in the manufacture of the product. Then the cost of building the proper curing room is much less under the factory system than in the private dairy. Conditions of temperature and moisture can readily be secured for a large amount of cheese cured together, that would practically be impossible if the same amount of cheese was distributed in ten or a dozen parcels and cured separately. In associated butter making, while the same ad- 
vantages hold true as in cheese making, they do not manifest themselves to the same degree. There is undoubtedly a considerable saving of labor and a vastly more uniform product where the milk of many patrons is manufactured into butter in a well equipped creamery under skilful supervision, but it is scarcely possible for a creamery handling the milk of many cows, seattered over a wide area and under the care of many persons, to make butter of so uniformly fine quality as is possible where not only the milk, from the time it is drawn until the finished product is sent to market, but the care and food of the cows as well, are under the same skilful supervision. One of the chief advantages of both the cheese and butter factory system is that it removes from the farm, and particularly from the farm home, a large amount of drudgery that in far too many cases fell upon those least able to bear it, the women of the household; so that while the butter of the very highest quality will probably for many years to come be made in relatively small individual or private dairies upon farms, still the factory system is increasing very rapidly, and will continue to do so until proportionately as much butter as cheese is made in factories.

Dairy legislation.-Dairy legislation in the United States has had two main objects. First, to secure to consumers of milk an unadulterated product. This has resulted in the establishment in many states of arbitrary legal standards for the quality of milk, and in others the passage of general laws prohibiting the 
adulteration of milk in any way (See Appendix C). Still, nearly one-third of the states have no laws whatever in regard to the sale of milk, though most of the large cities in these states have adopted municipal regulations of the same general nature as the state laws.

The second object of dairy legislation has been to guarantee the quality of a dairy product or to prevent the sale of a spurious product for a genuine one. The introduction of the manufacture of artificial butter or oleomargarine has led to both national and state regulation. The manufacture of oleomargarine, and particularly its sale as genuine butter, caused a great injury to the manufacturers of the genuine product, both in the sale of their goods and the prices received for the same. The state of New York was one of the first, if not the very first, to enact regulations controlling the sale of the imitation product, and in 1884 passed a law prohibiting the manufacture and sale of imitation butter within the limits of the state. This law was the subject of much litigation, but has been upheld by the state and national courts. It has been pretty thoroughly enforced, and is still in operation. In 1886 the National Government passed a law imposing an income tax of two cents per pound upon all imitation butter made in the country, and levying special license taxes upon those engaged in its manufacture and sale. This has resulted in a measurable control of the product, and with the supplemental laws that have been passed in a large number of the states the matter is now under 
careful and strict regulation. These laws have not resulted, as was fondly hoped by many dairymen, in absolutely stopping the manufacture of imitation butter, but have resulted in a great improvement in the quality of the imitation goods and in securing innocent purchasers against fraud in palming off upon them a spurious for a genuine article. At the same time, the cheaper imitation butter has crowded out of the market the poorer grades of genuine butter, and so improved the general average quality of genuine butter.

More recently the manufacture of so-called filled cheese has been regulated in the same way, by the passage by the National Congress, in 1896, of a law similar to the oleomargarine act, placing a revenue tax upon all filled cheese manufactured, and a license tax upon the manufacturers and dealers. This filled cheese is made from skimmed milk, to which has been added a certain amount of neutral animal fat.

Within the past twenty years the practice of making cheese from skimmed or partially skimmed milk has spread widely throughout the United States. The result has been that the reputation of American cheese has greatly fallen in the market, both abroad and at home. A remedy has been sought by the passage in several states, notably New York and Wisconsin, of laws authorizing the use, on cheese made from whole milk, of "State Brands" guaranteeing the quality of cheese so branded. While cheese-makers were at first not inclined to take advantage of these laws, 
the "State Brands" are now extensively used with very gratifying results as to the reputation of cheese so branded in the general markets.

Recently legislative control has been sought concorning still another product. A large business has grown up in gathering together, usually from country merchants who have taken the goods in trade, large amounts of poorly made butter and butter that has been spoiled or partially spoiled. The butter so collected is all melted up together, the solid impurities filtered out and the fat clarified by various processes that are kept more or less secret. The clarified fat is then churned with fresh skimmed milk and the resulting butter colored, salted and worked in the usual way. In some cases the better grades of butter collected from country stores are merely reworked and uniformly colored. All such butter, whatever the treatment it has received, is known as renovated or process butter, and is sold under the names "factory" and "imitation creamery." It is very much improved over the original butter from which it was prepared, which is often entirely unsalable as butter, but it is distinctly inferior to the better grades of fresh butter and injures their sale to a greater or less extent. For this reason several states have passed laws requiring that all butter that has been treated as described shall be distinctly branded "Renovated" butter.

Dairy markets.-In no one particular has the dairy industry developed in recent years more than in the 
line of production throughout the year. Formerly almost the whole product was made during the warm - months. This is measurably so stili in the case of cheese, but the demand has been constantly increasing for fresh butter the year round, and at the present time a fairly large proportion of the whole output is made during the winter months, the fresh butter commanding anywhere from two to ten cents per pound more than equally good butter that has been held in storage for several months. Very recently, with improvements in the methods of refrigeration and cold storage, the price of stored butter of the highest quality is reaching nearer to that of the fresh made goods, but the best consumers still continue to call for a fresh article. 



\section{APPENDIX}

\section{A. USEFUL RULES AND TESTS}

\section{Comparison of Thermometer Scales}

Centigrade Scale-Freezing point of water $=0$ Boiling point of water $=100$

Difference 100 degrees.

Fahrenheit Scale-Freezing point of water $=32$ Boiling point of water $=212$

$$
\begin{aligned}
& \text { Difference } 180 \text { degrees } \\
100 \text { degrees } \mathrm{C} . & =180 \text { degrees } \mathrm{F} . \\
5 & =9
\end{aligned}
$$

To Change Degrees Centigrade to Equivalent Degrees FAHRENHEIT

Multiply by $\frac{9}{5}$ and add 32 ; e. g.: $65^{\circ} \mathrm{C} . \times \frac{9}{5}=117+32=149^{\circ} \mathrm{F}$.

To Change Degrees Fahrenheit to Equivalent Degrees Centigrade

Subtract 32 and multiply by $\frac{5}{9} ;$ e. g.: $98^{\circ}$ F. $-32=66 \times \frac{5}{9}=37^{\circ}$ - C.

\section{To Find the Specific Gravity by the "Board of Health" Lactometer}

Multiply the reading by .29 , divide by 1,000 , and add $1 . ;$ e. g.: Observed reading, $94 \times .29=27.26 \div 1,000=.02726+1 .=1.027+$ specific gravity. 
To Change "Board of Health" Lactometer Degrees to Equivalent Quevenne Degrees

Multiply the "Board of Health" reading by ..29; e. g.: "Board of Health" reading, $105 \times .29=30.45=30+$ Quevenne reading.

\section{To Change Quevenne Lactometer. Degrees to Equivalent "Board of Health" Degrees}

Divide the observed Quevenne reading by .29; e. g.: Quevenne reading, $34 \div .29=117$. + ordinary or "Board of Health" reading.

\section{Temperature Correction for Lactometer}

For Quevenne lactometer, .1 lactometer degree for each degree of temperature $\mathrm{F}$.

For ordinary or "Board of Health" lactometer, 1 lactometer degree for each 3 degrees of temperature $\mathrm{F}$.

To be added if the temperature is higher, or subtracted if it is lower than the standard of the lactometer.

To be used only when the temperature variation is less than 10 degrees from the standard of the lactometer.

To Estimate Solids Not Fat, and Total Solids, from the Specific Gravity and Per Cent of Fat

The following formulæ may be used:

Babcock (1) $\mathrm{S}=\frac{\mathrm{L}+.7 \mathrm{f}}{3.8}$

Babcock (2) $\mathrm{S}=\frac{\mathrm{L}}{4}+.2 \mathrm{f}$

Richmond (3) $\mathrm{T}=\frac{\mathrm{L}}{4}+\frac{6 \mathrm{f}}{5}+.14$

In the above $\mathrm{L}=$ corrected Quevenne lactometer reading, $f=$ per cent of fat, $\mathrm{S}=$ solids not fat; $\mathrm{T}=$ total solids; solids not fat + fat $=$ total solids.

To apply the above formulæ, if the percentage of fat is 4.2 and the lactometer reading at $60^{\circ} \mathrm{F}$. is 32 , then

(1) $\mathrm{S}=\frac{\mathrm{L}+.7 \mathrm{f}}{3.8}, \mathrm{~S}=\frac{32+.7(4.2)}{3.8}$

.7 of $4.2=2.94+32=34.94 \div 3.8=9.19=$ solids not fat.

$9.19+4.2=13.39=$ total solids 
(2) $\mathrm{S}=\frac{\mathrm{L}}{4}+.2 \mathrm{f}, \mathrm{S}=\frac{32}{4}+2 .(4.2)$

$32 \div 4=8, .2(4.2)=.84,8+.84=8.84=$ solids not fat.

$8.84+4.2=13.04=$ total solids.

(3) $\mathrm{T}=\frac{\mathrm{L}}{4}+\frac{6 \mathrm{f}}{5}+.14, \mathrm{~T}=\frac{32}{4}+\frac{6(4.2)}{5}+.14$

$32 \div 4=8,6 \times 4.2=25.2 \div 5=5.04$.

$8+5.04+.14=13.18=$ total solids.

\section{Litmus Test}

A method of determining whether a liquid is acid or alkaline.

In acid solutions, blue litmus turns red.

In alkaline solutions, red litmus turns blue.

\section{Phenolphthalein Test}

A method of determining whether a liquid is acid or alkaline.

In acid solutions, if phenolphthalein is added no change in color is produced.

In alkaline solutions, if phenolphthalein is added the liquid turns pink.

To Determine the Percentage of Lactic Acid in Milk bx the Use of Decinormal Alkali, or Farrington's Alkaline

TABLETS

Each c. c. decinormal alkali neutralizes .009 grams lactic acid. Therefore, multiply the number of c. c. decinormal alkali used by .009 and divide the product by the number of grams of milk taken. (Grams $=$ c. c. $\times 1.032$ ); e. g.: 20 c. c. of milk require 9 c. c. decinormal alkali to neutralize the acid. The per cent of acid is-

$.009 \times 9=.081$ grams lactic acid.

$.081 \div 20.64=.0039$, or $.39 \%$.

Each alkaline tablet $=3.8$ c. c. decinormal alkaii. Each tablet is dissolved in 10 c. c. water. Each c. c. tablet solution $=.38$ c. c. decinormal alkali. Therefore, each c. c. tablet solution will neutralize $.009 \times .38=.0034$ grams lactic acid. Therefore, multiply the number of c. c. of tablet solution used by .0034 and divide by the number of grams of milk taken (grams $=$ c. $\mathrm{c} \times 1.032) ; e . g$. :

20 c. c. of cream require 35 c. c. of tablet solution to neutralize the acid. The per cent of acid is-

$.0034 \times 35=.119$ grams lactic acid.

$.119 \div 20.64=.0058$, or $.58 \%$. 


\section{To Determine Lactic Acid, Using 17.6 c. c. of Milk or Cream}

Instead of determining lactic acid as described above, it is often more convenient to make the tablet solution of such a strength that 1 c. c. of the solution will neutralize .01 per cent of lactic acid in the amount of milk or cream taken. Since a 17.6 c. c. pipette is always found where there is a Babcock testing outfit, 17.6 c. c. is a very convenient assay, as it does not necessitate procuring an additional pipette. If 5 Farrington tablets are dissolved in 97 c. c. of water the solution will be of such a strength that each c. c. of solution will neutralize .01 per cent of lactic acid in 17.6 c. c. of milk, and the total number of c. c. used will indicate the amount of lactic acid present in hundredths of one per cent; e. $g$. :

5 tablets are dissolved in 97 c. c. water, and 35 c. c. of the solution are required to neutralize the lactic acid in 17.6 c. c. of cream; the percentage of lactic acid present is therefore .35 per cent.

\section{To Select Milk for Pasteurization}

Dissolve any convenient number of Farrington alkaline tablets in an equal number of ounces of water. Provide any convenient small measure, and to one measure of milk add two measures of the prepared tablet solution. If the milk remains uncolored, it contains more than .2 of 1 per cent of acid, and is too sour to be safely used. If it is colored pink, it contains less than .2 of 1 per cent of acid, and may safely be used for pasteurizing or sterilizing. Or, the tablet solution will be of very nearly the same strength, and may be used in the same way, if 3 tablets are dissolved in 90 c. c. of water.

\section{To Prepare Viscogen for Restoring the Consistency of Pasteurtzed Cream}

Two and one-half parts by weight of a good quality of granulated sugar are dissolved in five parts of water, and one part of quick lime gradually slaked in three parts of water. The resulting milk of lime is strained and added to the sugar solution. The mixture should be agitated at frequent intervals, and after two or three hours allowed to settle until the clear liquid can be decanted off. This clear liquid (viscogen) is the part used and should be kept in well-stoppered bottles, as it loses strength and becomes dark-colored when exposed to the air. The darkening in color, however, does not impair its usefulness. 


\section{To Standardize Cream and Milk (Pearson)}

When cream is being sent to the city trade or used for certain other purposes, it is often desired to have it contain a certain percentage of fat and the same each day. It is difficult to adjust a separator to skim cream always of the same richness and it has become a more or less common practice to skim a heavy cream and "standarize" it. This is done by mixing with it enough of the skimmed milk that has just been removed, or whole milk or thinner cream, to reduce the fat content to the proper point.

The following is an easy and accurate way to determine the quantity of skimmed milk, milk, or thin cream that must be added to a rich cream to produce a cream of required richness or fat content:

Draw a rectangle and write at the two left-hand corners the percentages of fat in the fluids to be mixed, and in the center, place the required percentage. At the upper right-hand corner put the number which represents the difference between the two numbers standing in line with it, $i . e$., the number in the center and the one at the lower left-hand corner. At the lower right-hand corner put the number that represents the difference between the two numbers in line with it. Now let the upper right-hand number refer to the upper left and the lower right-hand to the lower left, then the two right-hand numbers show the relative quantities of the fluids represented at the left-hand corners that must be combined to give a fluid of the desired standard which is represented in the center.

For example, suppose we have cream testing 39 per cent fat, and we wish to standardize this to a 30 per cent cream by the use of milk testing, 5 per cent fat. Following the directions we have this diagram-

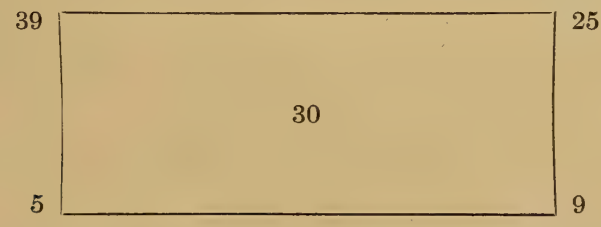

which shows at once that 39 per cent cream and 5 per cent milk must be combined in the proportion of 25 to 9 in order to produce 30 per cent cream. If we wish to use 50 pounds of 39 per cent cream, then 18 pounds of 5 per cent milk must be added, as is shown by this proportion.

$$
25: 9:: 50: 18
$$


Suppose we wish to find the proper amounts of the rich cream and milk to produce 170 pounds of 30 per cent cream:

If we take 25 pounds of the 39 per cent cream and 9 pounds of the 5 per cent milk, as is shown by the diagram, we will have a total of 34 pounds, but we want a total of 170 pounds, or five times as nuch; then we must take five times as much of each of the ingredients, or 125 pounds of 39 per cent cream and 45 pounds of the milk, as is shown by these proportions:

$$
\begin{aligned}
& 34: 25:: 170: 125 \\
& 34: 9:: 170: 45
\end{aligned}
$$

In the same manner milks of different quality may be standardized without going to the trouble of mixing them all together in one large vat.

If it is wanted to mix the milks from two dairies testing 4.9 per cent fat and 3.5 per cent to produce a 4.6 per cent milk, the diagram shows these milks must be mixed in the proportion of 1.1 to .3 or 11 to 3 . Thus:

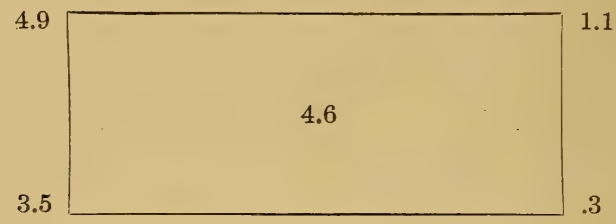

If we have 120 pounds of the 4.9 per cent milk we must mix with it 32.7 pounds of 3.5 per cent milk, as is shown by this proportion:

$$
11: 3:: 120: 32.7
$$

To Prepare Artificial "Starter" or Ferment for Ripening Milk OR CREAM*

A starter is a material containing desirable bacteria for the ripening or souring of dairy products. Originally it may be a "commercial culture" or it may be taken from a sample of clean sound sour milk or buttermilk from any local source. This material in the commercial culture may be a preparation of meat broth, milk, or other substances. It may be propagated by the dairyman or creameryman in skimmed milk or whole milk and, in unusual cases, in other media.

*From circular No. 10, Cornell Cniversity Agricultural Experiment Station. 


\section{STEPS IN PROPAGATION}

1. Take three one-quart milk bottles or fruit jars. Glass is preferable, as it allows the operator to see when all of the dirt has been removed, and the condition of the curd can easily be inspected through the transparent wall. Three bottles should be employed, for in heating glass is likely to break; and it is always well to have a sufficient number of containers from which to choose.

2. Use fresh, clean milk, which must have a nice flavor. It may be either whole milk or skimmed milk. Usually it is advisable to use whole milk, for it is easier to choose desirable samples before milk has passed through the separator than afterward.

3. Fill the containers one-half to two-thirds full of milk. If they are filled full, it is difficult to prevent contamination from the covers, which are hard to sterilize when the pasteurization is done in hot water. Protect the containers with regular covers (caps or tops) or with glass tumblers.

4. Pasteurize by heating to $180^{\circ}$ to $200^{\circ} \mathrm{F}$. for 30 minutes or longer. A temperature of $150^{\circ} \mathrm{F}$. kills all sporeless bacteria. Higher temperatures up to $212^{\circ} \mathrm{F}$. do not kill the spores, but they are so weakened by the higher heat that they germinate more slowly and their harmful effect is retarded. This fact and the results of experience indicate a temperature of about $185^{\circ} \mathrm{F}$. to $200^{\circ} \mathrm{F}$. as best. The heating and cooling can be done in cans immersed in water. Stirring hastens the processes, but is not necessary when the heating surface is not hotter than about $200^{\circ} \mathrm{F}$. Where the heating is done by steam, stirring is necessary to prevent scorching.

After heating, cool to a ripening temperature of $60^{\circ}$ to $75^{\circ} \mathrm{F}$. Pasteurization may be accomplished by tying a string about the necks of the bottles and suspending them in a pail or vat heated by steam, or in a kettle or dish heated on a stove. (If pasteurized over a fire, do not let bottles rest on the bottom of receptacle.) Other supports may be used to keep the containers from tipping over. The temperature should be raised and reduced slowly to prevent breaking the glass.

5. After pasteurization the milk is ready for inoculation. Inoculate in a quiet place where the wind cannot blow dirt and bacteria into this clean seed bed. With dry fingers remove the cover and place it in a bacterially clean spot, as in a recently scalded dipper. Pour in all of the commercial culture, or 2 to 10 per cent from the previous day's culture. Be sure that the curd from the previous day is well broken. After inoculation, shake the freshly inoculated sample to distribute the bacteria. 
6. Incubate at about $60^{\circ}$ to $75^{\circ} \mathrm{F}$. The first inoculation from the commercial culture should be incubated at about $70^{\circ}$ to $85^{\circ} \mathrm{F}$. The small inoculations require higher temperatures than the large inoculations. By experience an operator can soon learn what inoculation and temperature to use to ripen his starter in a given time. Usually a 6 to 8 per cent inoculation will ripen a starter in twelve hours at about $65^{\circ} \mathrm{F}$. The temperature must be fairly constant.

7. The starter is ripe when a curd forms. This curd should be soft and like custard in appearance. It should not be hard and firm.

8. After the starter is ripe, hold it at $50^{\circ} \mathrm{F}$. or a few degrees lower until time to use. For best results a starter should not be held longer than a few hours. However, it may be held two or three days and not be badly over-ripened. Do not shake the starter before putting it in storage.

9. Upon examination the curd should be smooth and compact, without gas pockets. Gas shows the presence of undesirable bacteria. A hard, lumpy curd, whey, and high acid show the over-ripe condition, which is very undesirable. After the condition of the curd is noted, shake well to break it into a smooth, lumpless condition. Shake with a rotary motion, being careful not to touch the cap for fear of contamination. Now smell and taste it, but never from the starter container. Always pour some of the curd into a spoon or cup, and then replace the cover immediately. After smelling, it is best to put at least a teaspoonful into the mouth. Seek for a desirable, clean, mild, acid flavor. The first propagation is likely to be somewhat disagreeable because of the presence of some of the original medium.

\section{GENERAL DIRECTIONS}

In a creamery or a large dairy it is necessary to carry more than a pint or a quart of starter. Along with the mother starter a second starter of ten to fifty pounds may be carried. After the mother starter in the glass container is inoculated, the remainder of the previous day's mother starter is poured into the second starter, and the cream is inoculated from the second starter. In large creameries, third and fourth starters are carried. Care should be taken in pasteurization not to cook the milk in these large amounts. In the mother starter this makes little difference.

It is necessary to use a larger inoculation from starter to cream than from starter to starter, because the seed bed is not so well prepared. The inoculation of the cream may vary from 8 to 50 per cent. 
Usually it is necessary to propagate the mother starter two or three times before the flavor of the commercial culture, which is often very disagreeable, will disappear.

A starter may be carried two to four weeks before it goes "off." Often it is carried several months, and often less than two weeks. This depends almost altogether on the carefulness of the operator.

\section{To Detect Ordinary Fermentations or "Taints" in Milk}

Procure as many test tubes one inch in diameter by five inches long as there are samples of milk to be tested, and a suitable rack to hold them in an upright position. Wash and rinse the tubes thoroughly and sterilize them by boiling in water for thirty minutes or by exposure to live steam in a sterilizing oven for fifteen minutes. After sterilizing they may be allowed to drain dry and then should be kept covered till wanted. When wanted for use the tubes should be filled one-half to two-thirds full with the suspected milk, closed with a piece of glass or plug of cotton and placed in the rack in water kept as nearly constant as possible at $100^{\circ} \mathrm{F}$. In from three to four hours the samples may be inspected without shaking or stirring. Gaseous fermentations will be manifested by the appearance of bubbles of gas upon the surface or throughout the mass, souring fermentations by coagulation of the milk and putrefactive fermentations or "taints" by various odors manifest to the nose when the covers are removed. The samples should be kept for at least twenty-four hours and examinations made at frequent intervals.

\section{The Wisconsin Curd Test}

This test is used for the same purpose as the fermentation test described above, and is made as follows: Procure as many covered pint glass jars as there are samples of milk to be tested and clean and sterilize them as described above. Fill each jar two-thirds full of the milk to be tested, label them plainly and put them up to the neck in a tub or vat of water heated to $98^{\circ} \mathrm{F}$. When the milk has reached the temperature of the water add ten drops of rennet extract to each jar, and mix it with the milk by shaking the jar. Allow the jars to stand until the curd is firm and then cut the curd finely with a case knife. When the curd has settled pour off the whey and allow the curd to settle again till a second portion of whey can be removed. The best tests are made when the removal of whey is as complete as possible. The jars containing the curd are then covered and again placed in the 
tub and the temperature maintained as nearly as possible at $98^{\circ} \mathrm{F}$. for six to twelve hours, when they may be examined. A solid, firm curd, without disagreeable odor or flavor, shows that the milk is pure and clean and has been properly handled. Impurities in the different samples are indicated by the presence of small round holes (gas cells) seen in the curd when it is cut across with a knife, or by various disagreeable odors that may be detected when the covers to the jars are removed. The apparatus for this test in an improved form is now furnished by most of the dairy supply houses.

\section{Monrad Rennet Test}

This test is figured and described on page 241 . It is used as follows: To determine the ripeness of milk for cheese making, put $5 \mathrm{c}$. c. commercial rennet in a $50 \mathrm{c}$. c. flask and fill with water to the mark. Put 200 c. c. of milk at $86^{\circ} \mathrm{F}$. in a suitable tin cup, and allow it to float in the vat. Add 5 c. c. of the diluted rennet, and note carefully the time required for the first appearance of coagulation. The time required will depend upon the amount of milk and rennet used, the strength of the rennet, the temperature and the ripeness of the milk. All except the last remaining constant from day to day, the degree of ripeness is measured by the time required for coagulation. The riper the milk the shorter the time; ordinarily from one and one-half to two minutes will be required. The diluted rennet must be made fresh every day.

\section{Marschall Rennet Test}

For a description and cut of this test, see page 242. Directions for its use: To determine the ripeness of milk for cheese making, fill the vessel to the 0 mark with milk, add a pipette full of commercial rennet, stir quickly, and place in such a position that the milk may flow freely from the orifice. When the milk ceases to flow, note the number of graduations exposed. The riper the milk the less the number of exposed graduations.

\section{Hot Iron Test}

A test used to indicate the maturity of curd in cheese making. The maturity is usually coincident with the amount of lactic acid present, so that the test is commonly called the hot iron test for acid.

The test is made by heating a bar of iron just short of redness, or so that it will hiss readily. A mass of curd is squeezed in the hand till all the whey possible is pressed out. The curd is then applied to the 
iron; the surface of the curd, melted by the heat, sticks to the iron, and the remainder is carefully and gently pulled away. If the curd is very immature, the melted part readily separates from that not affected by the heat, but when more mature, numerous fine threads of casein are drawn out when the two parts are separated, the length of these threads depending upon the maturity of the curd, and commonly measured in terms of acid, thus, $\frac{1}{4}$-inch acid, 2 -inch acid, etc.

\section{To Calibrate or Test the Accuracy of Graduation of Glassware Used in the Babcock Test}

Observe that the graduations are at equal distances apart. Fill the bottle carefully to the 0 point with clean rain water, wipe out the neck carefully, and add from a pipette or burette 2 c. c. water. It should fill the bottle exactly to the 10 point. Or, weigh the bottle filled with clean rain water to the 0 point with delicate balances, fill to the 10 point and weigh again. The difference in weight should be 2 grams. Or, into a dry, empty bottle put 2 c. c. or 27.18 grams mercury, insert a tight-fitting plug carefully to the 10 point, and invert the bottle; the mercury should just reach to the 0 point. The pipette should hold 17.6 grams of water, or 239 grams of mercury. Any piece showing a discrepancy of 2 per cent should be discarded.

\section{To Test Butter or Cheese with the Babcock Test}

Weigh out on a balance sensitive to .1 gram, from 4 to 8 grams of the substance to be tested. Divide into small pieces and put into an ordinary test bottle, or bottle with detachable neck, with about 10 c. c. of warm water. Add the acid, and complete the test in the ordinary way. The percentage of fat will be found by the following proportion:

Weight of sample: 18 :: observed reading : per cent of fat; or

$18 \times$ observed reading weight of sample $=$ per cent of fat;

e. g.: 4.8 grams of cheese tested, showed a reading of 9.2 per cent fat in the test bottle. The cheese contained:

$$
\frac{18 \times 9.2}{4.8}=34.5 \text { per cent fat. }
$$

The Cornell Butter Moisture-Test

The apparatus used in the Cornell moisture-test is an alcohol lamp, stand, asbestos sheet, hot-pan lifter, aluminum cup for holding the 
sample, and a special moisture scale. The scale is specially adapted for moisture work, but may be used as a cream scale in operating the Babcock test.

The scale has a tare weight for balancing the cup and a large and small weight for weighing the sample and obtaining the percentage of moisture. The beam has two rows of figures which give readings with the larger weight. The lower row gives readings in grams and the upper row in percentages. The smaller weight gives readings in grams when the weight is moved from 1 forward. Each notch represents .02 grams, the total value of the small scale being .2 grams. When the small weight is moved from 0 backward, each notch represents a loss of .1 per cent of moisture when 20.2 grams of butter are used. The small weight is intended to be used only in moisture work. In using the scale for Babcock work, the small weight is not used but is left at rest on the figure 1. Then, when the scales are balanced, the small weight is negligible. Care must be taken not to let any draft of air, as from an open window, strike the scales when in use, as they are so sensitive that a very slight current of air would throw them out of balance. The scales will give readings in percentages only when 20.2 grams of butter have been weighed or, in other words, when the large weight is on 20 (of the gram scale) and the small weight is on zero.

The cup used is of cast aluminum and is durable and perfectly smooth. The absence of creases or crevices allows it to be cleaned and dried thoroughly.

Operation of the test.-It is necessary that a representative sample be taken for a moisture-test. If the butter is sold in tubs, the sample should be taken from the tub with a butter trier, after the butter has been packed. It is best to take three drawings-one from near the edge, one from the middle, and one half way between the edge and the middle. Some butter-makers test the butter as soon as it is worked. This is a mistake, since considerable moisture is lost in the process of printing and packing.

Place the sample to be tested in a glass container which has a fairly wide mouth, so that the sample can be stirred. A quart fruit jar is useful for this purpose. Then hold the container in warm water until the butter begins to melt. Remove the container from the warm bath and thoroughly mix the melted with the unmelted butter. In the laboratory a long-bladed cheese knife was found very useful for mixing the butter. A wooden stirrer should not be used, as it is likely to take up moisture from the sample. The process of melting the butter and mixing it with the unmelted butter is repeated until the sample con- 
tains no lumps and the entire mass is about the consistency of thick cream. The container is then transferred to cold water and the sample thoroughly mixed as the butter cools. There is a tendency for the fat around the outside of the container to harden rapidly and force the water toward the center of the jar. For this reason special care must be taken to keep the butter scraped off the sides of the container and thoroughly mixed with the softer butter in the center of the jar. When the sample is all of about the texture of ordinary butter the mixing may be stopped. If the process has been properly done, the water will be evenly distributed throughout the sample and any desired amount of the latter may be removed for testing.

After the cup is thoroughly cleaned and dried, it is placed on the scales and balanced by means of the tare weight on the round bar attached to the beam of the scales. The large weight should rest on the zero mark (of the gram scale) and the small weight on 1 while the cup is being balanced. The cup should not be balanced until it is about the same temperature as that of the room. After the cup is balanced, the larger weight is moved to the 20 mark (of the gram scale) and the small weight to the zero mark. Butter from the prepared sample is then added to the cup until the scales are accurately balanced. The alcohol lamp is then placed under the iron stand and the asbestos sheet placed on the stand. The lamp is lighted and the cup placed on the asbestos sheet. It is well to light the lamp at least two or three minutes before placing the cup on the asbestos in order to heat the asbestos and save time. T.e heat of the flame may be increased or diminished by raising or lowering the wick. The cup should always be handled with the hot-pan lifter, as by so doing it will be kept clean and errors in weight due to dirt on the cup will be avoided.

While the sample is heating it should be shaken from time to time, as this breaks up the blanket of casein on the surface and hastens the escape of moisture. As soon as the casein has lost its snow-white color the cup should be removed from the flame. When the moisture has all been driven from the sample, a slightly pungent odor may be noticed. This may also be used as a guide to tell when the sample has been heated enough. The foam begins to subside at this point. Often one or two small pieces of casein are slow to give up their moisture. This is indicated by the snow-white color of the pieces. Evaporation can be hastened by shaking the sample with a rotary motion and thoroughly mixing these pieces with the hot liquid. If this is not done, one might have to heat the sample so long that some of the fat, which had already given up its moisture, would volatilize. 
After all the moisture is driven off, the sample is allowed to cool to room temperature. While cooling, the cup should be covered with something (a sheet of paper will do) to prevent the sample taking up moisture from the atmosphere. After cooling, the cup is placed on the scales. The sample is lighter than before heating, because it has lost its moisture. The bar of the scales will therefore remain down. The weights are then reversed until the scales just balance.

Each notch that the larger weight is reversed has a value of 1 per cent (reading on the upper scale), and each notch that the smaller weight is reversed has a value of .1 per cent. If, for example, after heating, the scales just balance when the larger weight rests on 15 (upper scale) and the smaller weight rests on .2, it would mean that the sample contained 15.2 per cent moisture.

It may be thought by those using the Cornell test for the first time that the use of the asbestos sheet is unnecessary. It is true that any one who is very familiar with moisture determinations may heat butter in a direct flame and get fairly accurate results. But the heat of a flame is so intense and butter volatilizes so easily that the use of the asbestos sheet is always advisable. 


\section{B. METRIC SYSTEM}

The rneter is the primary unit of length. It is equal to $\frac{1}{1000000}$ th part of the distance measured on a meridian of the earth from the equator to the pole, and equals about 39.37 inches.

\begin{tabular}{|c|c|c|c|c|}
\hline \multicolumn{3}{|c|}{ Measures of Lengti. } & \multicolumn{2}{|r|}{ EQUIVALENTS. } \\
\hline $\begin{array}{l}\text { Myriameter } \\
\text { Kilometer } \\
\text { Hectometer } \\
\text { Dekameter } \\
\text { Meter } \\
\text { Decimeter } \\
\text { Centimeter } \\
\text { Millimeter }\end{array}$ & $\begin{array}{c}10,000 \\
1,000 \\
100 \\
10 \\
1 \\
.1 \\
.01 \\
.001\end{array}$ & $\begin{array}{c}\text { meters } \\
\text { " } \\
\text { "6 } \\
\text { "6 } \\
\text { meter } \\
\text { " } \\
\text { " }\end{array}$ & \{ & $\begin{array}{l}6.2137 \text { miles } \\
0.62137 \text { mile, or } \\
3280 \mathrm{ft} .10 \mathrm{in} . \\
328 \mathrm{ft} .1 \mathrm{in} . \\
393.7 \mathrm{in} . \\
39.37 \mathrm{in} . \\
3.937 \mathrm{in} . \\
.3937 \mathrm{in} . \\
.03937 \mathrm{in} .\end{array}$ \\
\hline
\end{tabular}

Measureg of Surface.

Hectare

Are

Centare
10,000 sq. meters

100 " " "

1 " meter
Equivalents.

2.471 acres

119.6 sq. yards

1550. sq. inches.
Measures of Capacity.

\begin{tabular}{|c|c|c|}
\hline & $\left|\begin{array}{l}\text { No. of } \\
\text { Liters. }\end{array}\right|$ & Cubic Measure. \\
\hline Kiloliter, or Stere & 1000 & 1 cr. meter \\
\hline Hectoliter & 100 & $.1 " “$ \\
\hline Dekaliter & 10 & $10 \mathrm{cu}$. decimeters \\
\hline Liter & 1 & 1 cu. decimeter \\
\hline Deciliter & .1 & .1 . \\
\hline Centiliter & .01 & 10 cu. centimeters \\
\hline Milliliter & .001 & $.1 \mathrm{cu}$. centimeter \\
\hline
\end{tabular}

Equivalents.

Dry Measure.

Liquid or Wine MeAsure.

$1.308 \mathrm{cu}$. yards 2 bu. 3.35 pks.

9.08 quarts .909 quart $6.1022 \mathrm{cu}$. inches $.6102 \mathrm{cu}$. inch .061 “" “
264.17 gal.

26.417 gal. 2.6417 gal. 1.0567 qts. .845 gill .388 H. oz. $.27 \mathrm{fl}$. dram 


\begin{tabular}{|c|c|c|c|}
\hline \multicolumn{2}{|c|}{ STSTEM OF Weights. } & \multicolumn{2}{|c|}{ EQUIVALENTS. } \\
\hline & No. of Grams. & $\begin{array}{l}\text { Weight of Water } \\
\text { Maximum Density. }\end{array}$ & $\begin{array}{l}\text { A TroIRDUPOIS } \\
\text { WEIGHT. }\end{array}$ \\
\hline Millier, or Tonnean & $1,000,000$ & 1 cu. meter & 2204.6 pounds \\
\hline Quintal & 100,000 & 1 hectoliter & 220.46 \\
\hline Myriagram & 10,000 & 1 dekaliter & 22.046 " \\
\hline Kilogram, or Kilo & 1,000 & 1 liter & 2.2046 \\
\hline Hectogram & 100 & 1 deciliter & $3.5274 \mathrm{oz}$. \\
\hline Dekagram & 10 & 10 cu. centimeters & .3527 "6 \\
\hline Gram & 1 & 1 cu. centimeter & 15.432 grains \\
\hline Decigram & .1 & .166 & $1.5432 " 6$ \\
\hline Centigram & .01 & 10 cu. millimeters & .1543 grain \\
\hline Milligram & .001 & 1 " millimeter & .0154 \\
\hline
\end{tabular}

\begin{tabular}{|c|c|c|c|}
\hline $\begin{array}{l}\text { Common- } \\
\text { Measure. }\end{array}$ & EQUIVALENTS. & $\begin{array}{l}\text { Common } \\
\text { MEasure. }\end{array}$ & EQUIVALENTS. \\
\hline $\begin{array}{l}\text { An inch } \\
\text { A foot } \\
\text { A yard } \\
\text { A rod } \\
\text { A mile } \\
\text { A sq. inch } \\
\text { A " foot } \\
\text { A " yard. } \\
\text { A " rod } \\
\text { An acre } \\
\text { A sq. mile } \\
\text { A cu. inch } \\
\text { A " toot }\end{array}$ & $\begin{array}{c}2.54 \text { centimeters } \\
.3048 \text { meter } \\
.9144 \text { " } \\
5.029 \text { meters } \\
1.6093 \text { kilometers } \\
6.452 \text { sq. centimeters } \\
.0929 \text { sq. meter } \\
.8361 \text { " " } \\
25.29 \text { sq. meters } \\
.4047 \text { hectare } \\
259 \text { hectares. } \\
16.39 \text { cu. centimeters } \\
.02832 \text { cu. meter }\end{array}$ & $\begin{array}{l}\text { A cu. yard } \\
\text { A cord } \\
\text { A liquid qt. } \\
\text { A gallon } \\
\text { A dry qt. } \\
\text { A peck } \\
\text { A bushel } \\
\text { An oz. aroirdupois } \\
\text { A pound "6 } \\
\text { A ton } \\
\text { A grain troy } \\
\text { An oz. " " } \\
\text { A pound " }\end{array}$ & $\begin{array}{l}.7646 \text { cu. meter } \\
3.624 \text { steres } \\
.9465 \text { liter } \\
3.786 \text { liters } \\
1.101 \text { "6 } \\
8.811 \text { “6 } \\
35.24 \text { "6 } \\
28.35 \text { grams } \\
.4536 \text { kilogram } \\
.9072 \text { tonneau } \\
.0648 \text { gram } \\
31.104 \text { grams } \\
.3732 \text { kilogram }\end{array}$ \\
\hline
\end{tabular}




\section{LEGAL STANDARDS FOR DAIRY PRODUCTS DAIRY LAWS}

\section{FEDERAL STANDARDS.}

By authority of the Act of Congress, approved March 3, 1903, and generally known as the "Pure Food Law" the Secretary of Agriculture is empowered "to establish standards of purity for food products and to determine what are regarded as adulterations therein." The following standards have been established for dairy products and are published in Circular No. 19 of the office of the Secretary of Agriculture under date of June 26, 1906. These standards are generally accepted throughout the United States and have been officially adopted by many of the states.

\section{Milk and Its Products \\ a. MILKS}

1. Milk is the fresh, clean, lacteal secretion obtained by the complete milking of one or more healthy cows, properly fed and kept, excluding that obtained within fifteen days before and ten days after calving, and contains not less than eight and one-half (8.5) per cent of solids not fat, and not less than three and one-quarter (3.25). per cent of milk fat.

2. Blended milk is milk modified in its composition so as to have a definite and stated percentage of one or more of its constituents.

3. Skim milk is milk from which a part or all of the cream has been removed and contains not less than nine and one-quarter (9.25) per cent of milk solids.

4. Pasteurized milk is milk that has been heated below boiling but sufficiently to kill most of the active organisms present and immediately cooled to $50^{\circ} \mathrm{F}$. or lower.

5. Sterilized milk is milk that has been heated at the temperature of boiling water or higher for a length of time sufficient to kill all organisms present.

6. Condensed milk, evaporated milk, is milk from which a considerable portion of water has been evaporated and contains not less than twenty-eight (28) per cent of milk solids of which not less than twentyseven and five-tenths (27.5) per cent is milk fat. 
7. Sweetened condensed milk is milk from which a considerable portion of water has been evaporated and to which sugar (sucrose) has been added, and contains not less than twenty-eight (28) per cent of milk solids, of which not less than twenty-seven and five-tenths (27.5) per cent is milk fat.

8. Condensed skim milk is skim milk from which a considerable portion of water has been evaporated.

9. Buttermilk is the product that remains when butter is removed from milk or cream in the process of churning.

10. Goat's milk, ewe's milk, et cetera, are the fresh, clean, lacteal secretions, free from colostrum, obtained by the complete milking of healthy animals other than cows, properly fed and kept, and conform in name to the species of animal from which they are obtained.

\section{b. CREAM}

1. Cream is that portion of milk, rich in milk fat, which rises to the surface of milk on standing, or is separated from it by centrifugal force, is fresh and clean and contains not less than eighteen (18) per cent of milk fat.

2. Evaporated cream, clotted cream, is cream from which a considerable portion of water has been evaporated.

\section{c. MILK FAT OR BUTTER FAT}

1. Milk fat, butter fat, is the fat of milk and has a Reichert-Meissl number not less than twenty-four (24) and a specific gravity not less than $0.905\left(\frac{40^{\circ} \mathrm{C} .}{40^{\circ} \mathrm{C} .}\right)$

\section{d. BUTTER}

1. Butter is the clean, non-rancid product made by gathering in any manner the fat of fresh or ripened milk or cream into a mass, which also contains a small portion of the other milk constitutents, with or without salt, and contains not less than eighty-two and five-tenths (82.5) per cent of milk fat. By acts of Congress approved August 2, 1886, and May 9, 1902, butter may also contain added coloring matter.

2. Renovated butter, process butter, is the product made by melting butter and reworking, without the addition or use of chemicals or any substances except milk, cream, or salt, and contains not more than 
sixteen (16) per cent of water and at least eighty-two and five-tenths (82.5) per cent of milk fat.

\section{e. CHEESE}

1. Cheese is the sound, solid, and ripened product made from milk or cream by coagulating the casein thereof with rennet or lactic acid, with or without the addition of ripening ferments and seasoning, and contains, in the water-free substance, not less than fifty (50) per cent of milk fat. By act of Congress, approved June 6, 1896, cheese may also contain added coloring matter.

2. Skim milk cheese is the sound, solid, and ripened product, made from skim milk by coagulating the casein thereof with rennet or lactic acid, with or without the addition of ripening ferments and seasoning.

3. Goat's milk cheese, ewe's milk cheese, et cetera, are the sound, ripened products made from the milks of the animals specified, by coagulating the casein thereof with rennet or lactic acid, with or without the addition of ripening ferments and seasoning.

\section{f. ICE CREAMS}

1. Ice cream is a frozen product made from cream and sugar, with or without a natural flavoring, and contains not less than fourteen (14) per cent of milk fat.

2. Fruit ice cream is a frozen product made from cream, sugar, and sound, clean, mature fruits, and contains not less than twelve (12) per cent of milk fat.

- 3. Nut ice cream is a frozen product made from cream, sugar and sound, nonrancid nuts, and contains not less than twelve (12) per cent of milk fat.

\section{g. MisCelliANEOUS MILK PRODUCTS}

1. Whey is the product remaining after the removal of fat and casein from milk in the process of cheese-making.

2. Kumiss is the product made by the alcoholic fermentation of mare's or cow's milk.

\section{STATE STANDARDS FOR MILK AND CREAM}

The following states and territories, viz., Alabama, Arizona, Arkansas, Delaware, Maryland, Mississippi, New Mexico, South Caro- 
lina, Tennessee and West Virginia have established no legal standard.

In the other states the percentage standards are as follows:

Milk

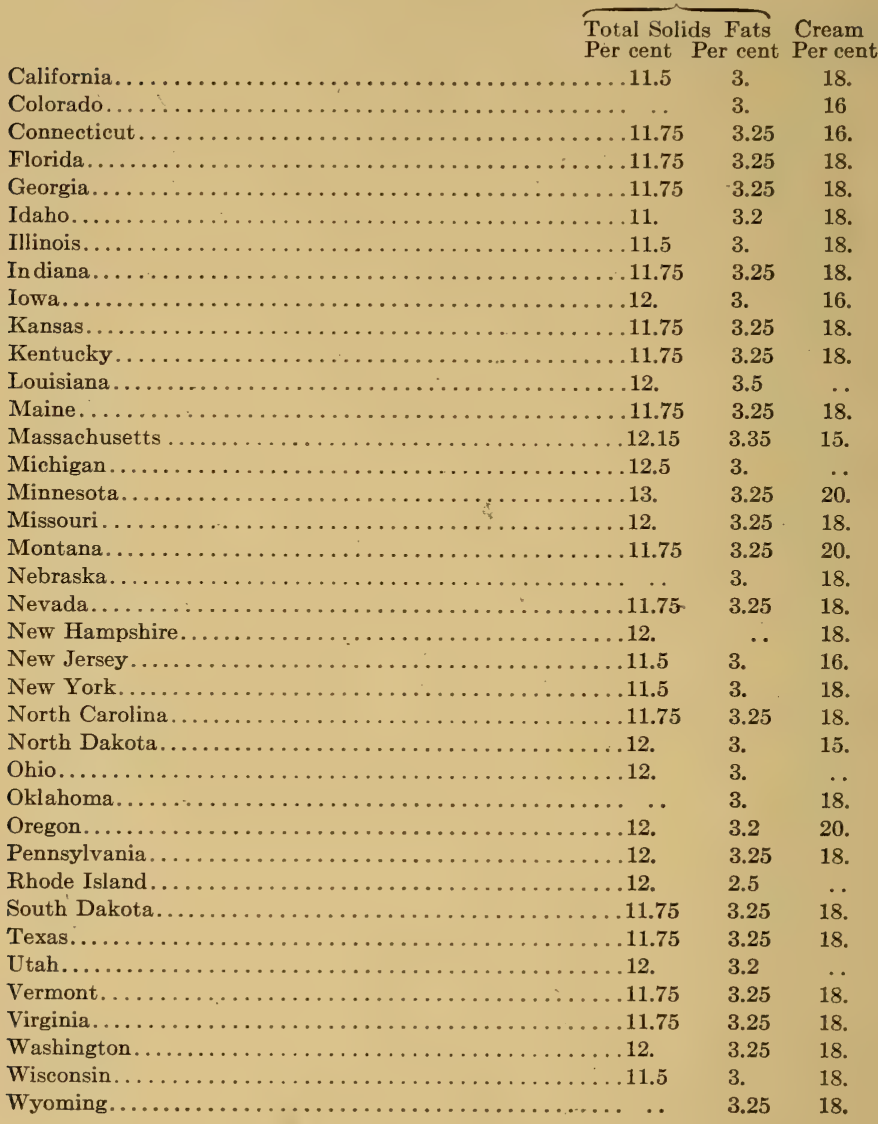




\section{The full texts of the National Oleomargarine Law} and the Filled Cheese Law are as follows:

\section{THE OLEOMARGARINE LAW}

[Act of August 2, 1886 (24 Stat., 209), as amended by acts of October 1, 1890 (26 Stat., 621), and May 9, 1902, to make oleomar garine and other imitation dairy products subject to the laws of any State, or Territory, or the District of Columbia into which they are transported, and to change the tax on oleomargarine, and to impose a tax, provide for the inspection, and regulate the manufacture and sale of certain dairy products, and to amend an act entitled "An act defining butter, also imposing a tax upon and regulating the manufacture, sale, importation, and exportation of oleomargarine," approved August 2, 1886.]

Be it enacted by the Senate and House of Representatives of the United States of America in Congress assembled, That all articles known as oleomargarine, butterine, imitation, process, renovated, or adulterated butter, or imitation cheese, or any substance in the semblance of butter or cheese not the usual product of the dairy and not made exclusively of pure and unadulterated milk or cream, transported into any State or Territory or the District of Columbia, and remaining therein for use, consumption, sale, or storage therein shall, upon the arrival within the limits of such State or Territory or the District of Columbia, be subject to the operation and effect of the laws of such State or Territory or the District of Columbia, enacted in the exercise of its police powers to the same extent and in the same manner as though such articles or substances had been produced in such State or Territory or the District of Columbia, and shall not be exempt therefrom by reason of being introduced therein in original packages or otherwise.

SEction 1, Act of August 2, 1886:

That for the purpose of this act the word "butter" shall be understood to mean the food product usually known as butter, and which is made exclusively from milk or cream, or both, with or without common salt, and with or without additional coloring matter.

SEC. 2, Act of August 2, 1886:

That for the purposes of this act certain manufactured substances, certain extracts, and certain mixtures and compounds, including such mixtures and compounds with butter, shall be known and designated as "oleomargarine," namely: All substances heretofore known as oleomargarine, oleo, oleomargarine-oil, butterine, lardine, suine and neutral; all mixtures and compounds of oleomargarine, oleo, oleomargarine-oil, butterine, lardine, suine, and neutral; all lard extracts and tallow extracts; and all mixtures and compounds of tallow, beef-fat, suet, lard, lard-oil, vegetable oil, annotto, and other coloring matter, intes- 
tinal fat, and offal fat made in imitation or semblance of butter, or when so made, calculated or intended to be sold as butter or for butter.

SEC. 3, Act of August 2, 1886, as amended by Section 2 of the Act of May 9, 1902, provides as follows:

That special taxes are imposed as follows:

Manufacturers of oleomargarine shall pay six hundred dollars. Every person who manufactures oleomargarine for sale shall be deemed a manufacturer of oleomargarine.

And any person that sells, vends, or furnishes oleomargarine for the use and consumption of others, except to his own family table without compensation, who shall add to or mix with such oleomargarine any artificial coloration that causes it to look like butter of any shade of yellow shall also be held to be a manufacturer of oleomargarine within the meaning of said Act, and subject to the provisions thereof.

Wholesale dealers in oleomargarine shall pay four hundred and eighty dollars. Every person who sells or offers for sale oleomargarine in the original manufacturer's packages shall be deemed a wholesale dealer in oleomargarine. But any manufacturer of oleomargarine who has given the required bond and paid the required special tax, and who sells only oleomargarine of his own production, at the place of manufacture, in the original packages to which the tax-paid stamps are affixed, shall not be required to pay the special tax of a wholesale dealer in oleomargarine on account of such sales.

Retail dealers in oleomargarine shall pay forty-eight dollars. Every person who sells oleomargarine in less quantities than ten pounds at one time shall be regarded as a retail dealer in oleomargarine. And sections thirty-two hundred and thirty-two, thirty-two hundred and thirty-three, thirty-two hundred and thirty-four, thirty-two hundred and thirty-five, thirty-two hundred and thirty-six, thirty-two hundred and thirty-seven, thirty-two hundred and thirty-eight, thirty-two hundred and thirty-nine, thirty-two hundred and forty, thirty-two hundred and forty-one, and thirty-two hundred and forty-three of the Revised Statutes of the United States are, so far as applicable, made to extend to and include and apply to the special taxes imposed by this section, and to the persons upon whom they are imposed: Provided further, That wholesale dealers who vend no other oleomargarine or butterine except that upon which a tax of one-fourth of one cent per pound is imposed by this Act, as amended, shall pay two hundred dollars; and such retail dealers as vend no other oleomargarine or butterine except that upon which is imposed by this Act, as amended, a tax of one-fourth of one cent per pound shall pay six dollars. 
SEC. 4, Act of August 2, 1886:

That every person who carries on the business of a manufacturer of oleomargarine without having paid the special tax therefor, as required by law, shall, besides being liable to the payment of the tax, be fined not less than one thousand and not more than five thousand dollars; and every person who carries on the business of a wholesale dealer in oleomargarine without having paid the special tax therefor, as required by law, shall, besides being liable to the payment of the tax, be fined not less than five hundred nor more than two thousand dollars; and every person who carries on the business of a retail dealer in oleomargarine without having paid the special tax therefor, as required by law, shall, besides being liable to the payment of the tax, be fined not less than fifty nor more than five hundred dollars for each and every offence.

SEC. 5, Act of August 2, 1886 :

That every manufacturer of oleomargarine shall file with the collector of internal revenue of the district in which his manufactory is located such notices, inventories, and bonds, shall keep such books and render such returns of material and products, shall put up such signs and affix such number to his factory, and conduct his business under such surveillance of officers and agents as the Commissioner of Internal Revenue, with the approval of the Secretary of the Treasury, may, by regulation, require. But the bond required of such manufacturer shall be with sureties satisfactory to the collector of internal revenue, and in a penal sum of not less than five thousand dollars; and the sum of said bond may be increased from time to time and additional sureties required at the discretion of the collector, or under instructions of the Commissioner of Internal Revenue.

SEC. 6, Act of August 2, 1886:

That all oleomargarine shall be packed by the manufacturer thereof in firkins, tubs, or other wooden packages not before used for that purpose; each containing not less than ten pounds, and marked, stamped, and branded as the Commissioner of Internal Revenue, with the approval of the Secretary of the Treasury, shall prescribe; and all sales made by manufacturers of oleomargarine, and wholesale dealers in oleomargarine shall be in original stamped packages. Retail dealers in oleomargarine must sell only from original stamped packages, in quantities not exceeding ten pounds, and shall pack the oleomargarine sold by them in suitable wooden or paper packages which shall be marked and branded as the Commissioner of Internal Revenue, with the approval of the Secretary of the Treasury, shall prescribe. Every person who knowingly sells or offers for sale, or delivers or offers to 
deliver, any oleomargarine in any other form than in new wooden or paper packages as above described, or who packs in any package any oleomargarine in any manner contrary to law or who falsely brands any package or affixes a stamp on any package denoting a less amount of tax than that required by law shall be fined for each offense not more than one thousand dollars, and be imprisoned not more than two years.

SEc. 7, Act of August 2, 1886:

That every manufacturer of oleomargarine shall securely affix, by pasting, on each package containing oleomargarine manufactured by him, a label on which shall be printed, besides the number of the manufactory and the district and State in which it is situated, these words: "Notice-The manufacturer of the oleomargarine herein contained has complied with all the requirements of law. Every person is cautioned not to use either this package again or the stamp thereon again, nor to remove the contents of this package without destroying said stamp, under the penalty provided by law in such cases." Every manufacturer of oleomargarine who neglects to affix such label to any package containing oleomargarine made by him, or sold or offered for sale by or for him, and every person who removes any such label so affixed from any such package, shall be fined fifty dollars for each package in respect to which such offense is committed.

Sec. 8, Act of August 2, 1886, as amended by Section 3, Act of May 9, 1902:

That upon oleomargarine which shall be manufactured and sold, or removed for consumption or use, there shall be assessed and collected a tax of ten cents per pound, to be paid by the manufacturer thereof; and any fractional part of a pound in a package shall be taxed as a pound: Provided, When oleomargarine is free from artificial coloration that causes it to look like butter of any shade of yellow said tax shall be one-fourth of one cent per pound. The tax levied by this section shall be represented by coupon stamps; and the provisions of existing laws governing the engraving, issue, sale, accountability, effacement, and destruction of stamps relating to tobacco and snuff, as far as applicable, are hereby made to apply to stamps provided for by this section.

SEC. 9, Act of August 2, 1886:

That whenever any manufacturer of oleomargarine sells, or removes for sale or consumption, any oleomargarine upon which the tax is required to be paid by stamps, without the use of the proper stamps, it shall be the duty of the Commissioner of Internal Revenue, within a period of not more than two years atter such sale or removal, upon satisfactory proof, to estimate the amount of tax which has been 
omitted to be paid, and to make an assessment therefor and certify the same to the collector. The tax so assesed shall be in addition to the penalties imposed by law for such sale or removal.

Sec. 10, Act of August 2, 1886:

That all oleomargarine imported from foreign countries shall, in addition to any import duty imposed on the same, pay an internal revenue tax of fifteen cents per pound, such tax to be represented by coupon stamps as in the case of oleomargarine manufactured in the United States. The stamps shall be affixed and canceled by the owner or importer of the oleomargarine while it is in the custody of the proper custom-house officers; and the oleomargarine shall not pass out of the custody of said officers until the stamps have been so affixed and canceled, but shall be put up in wooden packages, each containing not less than ten pounds, as prescribed in this act for oleomargarine manufactured in the United States, before the stamps are affixed; and the owner or importer of such oleomargarine shall be liable to all the penal provisions of this act prescribed for manufacturers of oleomargarine manufactured in the United States. Whenever it is necessary to take any oleomargarine so imported to any place other than the public stores of the United States for the purpose of affixing and canceling such stamps, the collector of customs, of the port where such oleomargarine is entered shall designate a bonded warehouse to which it shall be taken, under the control of such customs officer as such collector may direct; and every officer of customs who permits any such oleomargarine to pass out of his custody or control without compliance by the owner or importer thereof with the provisions of this section relating thereto, shall be guilty of a misdemeanor, and shall be fined not less than one thousand dollars nor more than five thousand dollars, and imprisoned not iess than six months nor more than three years. Every person who sells or offers for sale any imported oleomargarine, or oleomargarine purporting or claimed to have been imported, not put up in packages and stamped as provided by this act, shali be fined not less than five hundred dollars nor more than five thousand dollars, and be imprisoned not less than six months nor more than two years.

SEC. 11, Act of August 2, 1886:

That every person who knowingly purchases or receives for sale any oleomargarine which has not been branded or stamped according to law shall be liable to a penalty of fifty dollars for each such offense.

SEC. 12, Act of August 2, 1886:

That every person who knowingly purchases or receives for sale any oleomargarine from any manufacturer who has not paid the special 
tax shall be liable for each offense to a penalty of one hundred dollars, and to a forfeiture of all articles so purchased or received, or of the full value thereof.

SEC. 13, Act of August 2, 1886:

That whenever any stamped package containing oleomargarine is emptied, it shall be the duty of the person in whose hands the same is to destroy utterly the stamps thereon; and any person who willfully neglects or refuses so to do shall for each such offense be fined not exceeding fifty dollars, and imprisoned not less than ten days nor more than six months. And any person who fraudulently gives away or accepts from another, or who sells, buys, or uses for packing oleomargarine, any such stamped package, shall for each such offense be fined not exceeding one hundred dollars, and be imprisoned not more than one year. Any revenue officer may destroy any emptied oleomargarine package upon which the tax-paid stamp is found.

SEC. 14, Act of August 2, 1886:

That there shall be in the office of the Commissioner of Internal Revenue an analytical chemist and a microscopist, who shall each be appointed by the Secretary of the Treasury, and shall each receive a salary of two thousand five hundred dollars per annum; and the Commissioner of Internal Revenue may, whenever in his judgment the necessities of the service so require, employ chemists and microscopists, to be paid such compensation as he may deem proper, not exceeding in the aggregate any appropriation made for that purpose. And such Commissioner is authorized to decide what substances, extracts, mixtures, or compounds which may be submitted for his inspection in contested cases are to be taxed under this act; and his decision in matters of taxation under this act shall be final. The Commissioner may also decide whether any substance made in imitation or semblance of butter, and intended for human consumption, contains ingredients deleterious to the public health; but in case of doubt or contest his decision in this class of cases may be appealed from to a board hereby constituted for the purpose, and composed of the Surgeon-General of the Army, the Surgeon-General of the Navy, and the Commissioner (now Secretary) of Agriculture; and the decisions of this board shall be final in the premises.

SEc. 15, Act of August ₹, 1886:

That all packages of oleomargarine subject to tax under this act that shall be found without stamps or marks as herein provided, and all oleomargarine intended for human consumption which contains ingredients adjudged, as hereinbefore provided, to be deleterious to 
the public health, shall be forfeited to the United States. Any person who shall willfully remove or deface the stamps, marks, or brands on a package containing oleomargarine taxed as provided herein shall be guilty of a misdemeanor, and shall be punished by a fine of not less than one hundred dollars nor more than two thousand dollars, and by imprisonment for not less than thirty days nor more than six months.

SEC. 16, Act of August 2, 1886:

That oleomargarine may be removed from the place of manufacture for export to a foreign country without payment of tax or affixing stamps thereto, under such regulations and the filing of such bonds and other security as the Commissioner of Internal Revenue, with the approval of the Secretary of the Treasury, may prescribe. Every person who shall export oleomargarine shall brand upon every tub, firkin, or other package containing such article the word "Oleomargarine," in plain Roman letters not less than one-half inch square.

SEc. 17, Act of August 2, 1886:

That whenever any person engaged in carrying on the business of manufacturing oleomargarine defrauds, or attempts to defraud, the United States of the tax on the oleomargarine produced by him, or any part thereof, he shall forfeit the factory and manufacturing apparatus used by him, and all oleomargarine and all raw material for the production of oleomargarine found in the factory and on the factory premises, and shall be fined not less than five hundred dollars nor more than five thousand dollars, and be imprisoned not less than six months nor more than three years.

SEc. 18, Act of August 2, 1886:

That if any manufacturer of oleomargarine, any dealer therein, or any importer or exporter thereof shall knowingly or willfully omit, neglect, or refuse to do, or cause to be done, any of the things required by law in the carrying on or conducting of his business, or shall do anything by this act prohibited, if there be no specific penalty or punishment imposed by any other section of this act for the neglecting, omitting, or refusing to do, or for the doing or causing to be done, the thing required or prohibited, he shall pay a penalty of one thousand dollars; and if the person so offending be the manufacturer of or a wholesale dealer in oleomargarine, all the oleomargarine owned by him, or in which he has any interest as owner, shall be forfeited to the United States.

SEC. 19, Act of August 2, 1886:

That all fines, penalties, and forfeitures imposed by this act may be recovered in any court of competent jurisdiction. 
SEc. 20, Act of August 2, 1886:

That the Commissioner of Internal Revenue, with the approval of the Secretary of the Treasury, may make all needful regulations for the carrying into effect of this act.

SEC. 21, Act of August 2, 1886:

That this act shall go into effect on the ninetieth day after its passage; and all wooden packages containing ten or more pounds of oleomargarine found on the premises of any dealer on or after the ninetieth day succeeding the date of the passage of this act shall be deemed to be taxable under section eight of this act, and shall be taxed, and shall have affixed thereto the stamps, marks, and brands required by this act or by regulations made pursuant to this act; and for the purpose of securing the affixing of the stamps, marks and brands required by this act, the oleomargarine shall be regarded as having been manufactured and sold, or removed from the manufactory for consumption or use, on or after the day this act takes effect; and such stock on hand at the time of the taking effect of this act may be stamped, marked, and branded under special regulations of the Commissioner of Internal Revenue, approved by the Secretary of the Treasury; and the Commissioner of Internal Revenue may authorize the holder of such packages to mark and brand the same and to affix thereto the proper tax-paid stamps.

SEc. 4, Act of May 9, 1902:

That for the purpose of this act "butter" is hereby defined to mean an article of food as defined in "An Act defining butter, also imposing a tax upon and regulating the manufacture, sale, importation, and exportation of oleomargarine," approved August second, eighteen hundred and eighty-six; that "adulterated butter" is hereby defined to mean a grade of butter produced by mixing, reworking, rechurning in milk or cream, refining, or in any way producing a uniform, purified, or improved product from different lots or parcels of melted or unmelted butter or butter fat, in which any acid, alkali, chemical, or any substance whatever is introduced or used for the purpose or with the effect of deodorizing or removing therefrom rancidity, or any butter or butter fat with which there is mixed any substance foreign to butter as herein defined, with intent or effect of cheapening in cost the product or any butter in the manufacture or manipulation of which any process or material is used with intent or effect of causing the absorption of abnormal quantities of water, milk, or cream; that "process butter" or "renovated butter" is hereby defined to mean butter which has been subjected to any process by which it is melted, clarified or refined and made to resemble genuine butter, always excepting "adulterated butter" as defined by this Act. 
That special taxes are imposed as follows:

Manufacturers of process or renovated butter shall pay fifty dollars per year and manufacturers of adulterated butter shall pay six hundred dollars per year. Every person who engages in the production of process or renovated butter or adulterated butter as a business shall be considered to be a manufacturer thereof.

Wholesale dealers in adulterated butter shall pay a tax of four hundred and eighty dollars per annum, and retail dealers in adulterated butter shall pay a tax of forty-eight dollars per annum. Every person who sells adulterated butter in less quantities than ten pounds at one time shall be regarded as a retail dealer in adulterated butter.

Every person who sells adulterated butter shall be regarded as a dealer in adulterated butter. And sections thirty-two hundred and thirty-two, thirty-two hundred and thirty-three, thirty-two hundred and thirty-four, thirty-two hundred and thirty-five, thirty-two hundred and thirty-six, thirty-two hundred and thirty-seven, thirty-two hundred and thirty-eight, thirty-two hundred and thirty-nine, thirty-two hundred and forty, thirty-two hundred and forty-one, and thirty-two hundred and forty-three of the Revised Statutes of the United States are, so far as applicable, made to extend to and include and apply to the special taxes imposed by this section and to the person upon whom they are imposed.

That every person who carries on the business of a manufacturer of process or renovated butter or adulterated butter without having paid the special tax therefor, as required by law, shall, besides being liable to the payment of the tax, be fined not less than one thousand and not more than five thousand dollars; and every person who carries on the business of a dealer in adulterated butter without having paid the special tax therefor, as required by law, shall, besides being liable to the payment of the tax, be fined not less than fifty nor more than five hundred dollars for each offense.

That every manufacturer of process or renovated butter or adulterated butter shall file with the collector of internal revenue of the district in which his manufactory is located such notices, inventories, and bonds, shall keep such books and render such returns of material and products, shall put up such signs and affix such number of his factory, and conduct his business under such surveillance of officers and agents as the Commissioner of Internal Revenue, with the approval of the Secretary of the Treasury, may by regulation require. But the bond required of such manufacturer shall be with sureties satisfactory to the collector of internal revenue, and in a penal sum of not less than 
five hundred dollars; and the sum of said bond may be increased from time to time and additional sureties required at the discretion of the collector or under instructions of the Commissioner of Internal Revenue.

That all adulterated butter shall be packed by the manufacturer thereof in firkins, tubs, or other wooden packages not before used for that purpose, each containing not less than ten pounds, and marked, stamped, and branded as the Commissioner of Internal Revenue, with the approval of the Secretary of the Treasury, shall prescribe; and all sales made by manufacturers of adulterated butter shall be in original stamped packages.

Dealers in adulterated butter must sell only original or from original stamped packages, and when such original stamped packages are broken the adulterated butter sold from same shall be placed in suitable wooden or paper packages, which shall be marked and branded as the Commissioner of Internal Revenue, with the approval of the Secretary of the Treasury, shall prescribe. Every person who knowingly sells or offers for sale, or delivers or offers to deliver, any adulterated butter in any other form than in new wooden or paper packages as above described, or who packs in any package any adulterated butter in any manner contrary to law, or who falsely brands any package or affixes a stamp on any package denoting a less amount of tax than that required by law, shall be fined for each offense not more than one thousand dollars and be imprisoned not more than two years.

That every manufacturer of adulterated butter shall securely affix, by pasting, on each package containing adulterated butter manufactured by him a label on which shall be printed, besides the number of the manufactory and the district and State in which it is situated, these words: "Notice.-That the manufacturer of the adulterated butter herein contained has complied with all the requirements of law. Every person is cautioned not to use either this package again or the stamp thereon, nor to remove the contents of this package without destroying said stamp, under the penalty provided by law in such cases." Every manufacturer of adulterated butter who neglects to affix such label to any package containing adulterated butter made by him, or sold or offered for sale for or by him, and every person who removes any such label so affixed from any such package shall be fined fifty dollars for each package in respect to which such offense is committed.

That upon adulterated butter, when manufactured or sold or removed for consumption or use, there shall be assessed and collected a tax of ten cents per pound, to be paid by the manufacturer thereof, and any fractional part of a pound shall be taxed as a pound, and that 
upon process or renovated butter, when manufactured or sold or removed for consumption or use, there shall be assessed and collected a tax of one-fourth of one.cent per pound to be paid by the manufacturer thereof, and any fractional part of a pound shall be taxed as a pound. The tax to be levied by this section, shall be represented by coupon stamps, and the provisions of existing laws governing engraving, issuing, sale, accountability, effacement, and destruction of stamps relating to tobacco and snuff, as far as applicable, are hereby made to apply to the stamps provided by this section.

That the provisions of sections nine, ten, eleven, twelve, thirteen, fourteen, fifteen, sixteen, seventeen, eighteen, nineteen, twenty, and twenty-one of "An Act defining butter, also imposing a tax upon and regulating the manufacture, sale, importation, and exportation of oleomargarine," approved August second, eighteen hundred and eightysix, shall apply to manufacturers of "adulterated butter" to an extent necessary to enforce the marking, branding, identification, and regulation of the exportation and importation of adulterated butter.

Sec. 5, Act of May 9, 1902:

All parts of an act providing for an inspection of meats for exportation, approved August thirtieth, eighteen hundred and ninety, and of an act to provide for the inspection of live cattle, hogs, and the carcasses and products thereof which are the subjects on interstate commerce, approved March third, eighteen hundred and ninety-one, and of amendment thereto approved March second, eighteen hundred and ninety-five, which are applicable to the subjects and purposes described in this section shall apply to process or renovated butter. And the Secretary of Agriculture is hereby authorized and required to cause a rigid sanitary inspection to be made, at such times as he may deem proper or necessary, of all factories and storehouses where process or renovated butter is manufactured, packed, or prepared for market, and of the products thereof and materials going into the manufacture of same. All process or renovated butter and the packages containing the same shall be marked with the words "Renovated Butter" or "Process Butter" and by such other marks, labels, or brands and in such manner as may be prescribed by the Secretary of Agriculture, and no process or renovated butter shall be shipped or transported from its place of manufacture into any other State or Territory or the District of Columbia, or to any foreign country, until it has been marked as provided in this section. The Secretary of Agriculture shall make all needful regulations for carrying this section into effect, and shall cause to be ascertained and reported from time to time the quantity and 
quality of process or renovated butter manufactured, and the character and the condition of the material from which it is made. And he shall also have power to ascertain whether or not materials used in the manufacture of said process or renovated butter are deleterious to health or unwholesome in the finished product, and in case such deleterious or unwholesome materials are found to be used in product intended for exportation or shipment into other States or in course of exportation or shipment he shall have power to confiscate the same. Any person, firm, or corporation violating any of the provisions of this section shall be deemed guilty of a misdemeanor and on conviction thereof shall be punished by a fine of not less than fifty dollars nor more than five hundred dollars or by imprisonment not less than one month nor more than six months, or by both said punishments, in the discretion of the court.

Sec. 6, Act of May 9, 1902:

That wholesale dealers in oleomargarine, process, renovated, or adulterated butter shall keep such books and render such returns in relation thereto as the Commissioner of Internal Revenue, with the approval of the Secretary of the Treasury, may, by regulation, require; and such books shall be open at all times to the inspection of any internal revenue officer or agent. And any person who willfully violates any of the provisions of this section shall for each such offense be fined not less than fifty dollars and not exceeding five hundred dollars, and imprisoned not less than thirty days nor more than six months.

SEC. 7, Act of May 9, 1902:

This act shall take effect on the first day of July, nineteen hundred and two.

Oleomargarine law constitutional.-Supreme Court decision in McCray vs. United States. Decided May 31, 1904.

The law, act May 9, 1902, imposing a tax of 10 cents a pound on oleomargarine artificially colored in imitation of butter and one-fourth of 1 cent per pound on uncolored oleomargarine is constitutional. The courts can not hold a tax void because it is deemed too high. Although the effect of the tax in question may be to repress the manufacture of artificially colored oleomargarine, it is not on that account a violation of fundamental rights. An act of Congress exerting the taxing power can not be avoided on the ground that it is an abuse of power. Whilst the statute recognized the right of a manufacturer to use any or all of the authorized ingredients so as to make oleomargarine, and also author- 
ızed as one of the ingredients butter artificially colored, if the manufacturer elected to use such ingredient last mentioned, and thereby gave to his manufactured product artificial coloration, such product so colored, although being oleomargarine, was not within the exception created by the proviso, and therefore came under the general rule subjecting oleomargarine to the tax of ten cents a pound. (Vol. 7, Treas. Dec. (1904), Decision No. 795. Supreme Court of the United States. No. 301. October Term, 1903. Leo W. McCray, plaintiff in error, vs. United States.)

Oleomargarine.-Opinion of Supreme Court in the palm-oil case.-Supreme Court of the United States.-No. 19-October term. 1904.-August Cliff, plaintiff in error, vs. United States, October 24, 1904.

One of the purposes of the oleomargarine legislation was to prevent the sale of oleomargarine as and for butter. When any substance, although named as a possible ingredient of oleomargarine, serves only the function of coloring the mass so as to cause it to "look like butter of any shade of yellow," it is an artificial coloration, and the product is subject to a tax of 10 cents per pound. (Treas. Dec. Nos. 615, 839.)

Regulations prescribed by the Commissioner of Internal Revenue, with the approval of the Secretary of the Treasury, in regard to marks and brands on packages of oleomargarine, are authorized by law. They are matters of detail confided to the Executive branch, the authority to make which is within the competency of the legislative branch to confer. Courts will take judicial notice of them. (Joseph Wilkins vs. United States (1899), 96 Fed. Rep., 837; Vol. 2, Treas. Dec., No. 21623.)

Such power delegated to the Commissioner, with approval of the Secretary, involves no unconstitutional delegation of power (1897). (In re Kollock, 165 U. S., 526, 43 Int. Rev. Rec., 170; Prather vs. United States, 9 Appeal Cases, D. C., 82.)

Taxes imposed by the oleomargarine law as amended.

SPECIAL TAXES

Manufacturers of oleomargarine........................ $\$ 600$

Wholesale dealers in oleomargarine colored artificially to look like butter... 480

Wholesale dealers in oleomargarine not artificially colored only.......... 200

Retail dealers in oleomargarine colored artificially to look like butter..... 48

Retail dealers in oleomargarine not artificially colored only ........... 6

Manufacturers of adulterated butter............................ 600

Wholesale dealers in adulterated butter..................... 480

Retail dealers in adulterated butter........................ 48

Manufacturers of process or renovated butter................. 50 
TAXES ON PRODUCT

Oleomargarine (artificially colored), 10 cents per pound.

Oleomargarine (not artificially colored), $\frac{1}{4}$ cent per pound.

Adulterated butter, 10 cents per pound.

Process or renovated butter, $\frac{1}{4}$ cent per pound.

\section{THE FILLED CHEESE LAW}

AN ACT defining cheese, and also imposing a tax upon and regulating the manufacture, sale, importation and exportation of "filled cheese."

Be it enacted by the Sen te and House of Representatives of the United States of America in Congress assembled, That for the purposes of this act, the word "cheese" shall be understood to mean the food product known as cheese, and which is made from milk or cream, and without the addition of butter, or any animal, vegetable, or other oils or fats foreign to such milk or cream, with or without additional coloring matter.

SECtion 2. That for the purposes of this act certain substances and compounds shall be known and designated as "filled cheese," namely: All substances made of milk or skimmed milk, with the admixture of butter, animal oils or fats, vegetable or any other oils, or compounds foreign to such milk, and made in imitation or semblance of cheese.

SEc. 3. That special taxes are imposed as follows:

Manufacturers of filled cheese shall pay four hundred dollars for each and every factory per annum. Every person, firm, or corporation who manufactures filled cheese for sale shall be deemed a manufacturer of filled cheese. Wholesale dealers in filled cheese shall pay two hundred and fifty dollars per annum. Every person, firm, or corporation who sells, or offers for sale filled cheese in the original manufacturer's packages for resale, or to retail dealers as hereinafter defined, shall be deemed a wholesale dealer in filled cheese. But any manufacturer of filled cheese who has given the required bond and paid the required special tax, and who sells only filled cheese of his own production, at the place of manufacture, in the original packages, to which the tax-paid stamps are affixed, shall not be required to pay the special tax of a wholesale dealer in filled cheese on account of such sales.

Retail dealers in filled cheese shall pay twelve dollars per annum. Every person who sells filled cheese at retail, not for resale, and for actual consumption, shall be regarded as a retail dealer in filled cheese, and sections thirty-two hundred and thirty-two, thirty-two hun- 
dred and thirty-three, thirty-two hundred and thirty-four, thirtytwo hundred and thirty-five, thirty-two hundred and thirty-six, thirtytwo hundred and thirty-seven, thirty-two hundred and thirty-eight, thirty-two hundred and thirty-nine, thirty-two hundred and forty, thirty-two hundred and forty-one, thirty-two hundred and forty-three of the Revised Statutes of the United States are, so far as applicable, made to extend to and include and apply to the special taxes imposed by this section and to the persons, firms, or corporations upon whom they are imposed: Provided, That all special taxes under this act shall become due on the first day of July in every year, or on commencing any manufacture, trade, or business on which said tax is imposed. In the latter case the tax shall be reckoned proportionately from the first day of the month in which the liability to the special tax commences to the first day of July following.

SEC. 4. That every person, firm, or corporation who carries on the business of a manufacturer of filled cheese without having paid the special tax therefor, as required by law, shall, besides being liable to the payment of the tax, be fined not less than four hundred dollars and not more than three thousand dollars; and every person, firm, or corporation who carries on the business of a wholesale dealer in filled cheese without having paid the special tax therefor, as required by law, shall, besides being liable to the payment of the tax, be fined not less than two hundred and fifty dollars, nor more than one thousand dollars; and every person, firm, or corporation, who carries on the business of a retail dealer in filied cheese without having paid the special tax therefor, as required by law, shall, besides being liable for the payment of the tax, be fined not less than forty nor more than five hundred dollars for each and every offense.

SEC. 5. That every manufacturer of filled cheese shall file with the collector of internal revenue of the district in which his manufactory is located such notices, inventories, and bonds, shall keep such books and render such returns of materials and products, shall put up such signs and affix such number to his factory, and conduct his business under such surveillance of officers and agents as the Commissioner of Internal Revenue, with the approval of the Secretary of the Treasury, may by regulation require. But the bond required of such manufacturer shall be with sureties satisfactory to the collector of internal revenue, and in a penal sum of not less than five thousand dollars; and the amount of said bond may be increased from time to time, and additional sureties required, at the discretion of the collector or under instructions of the Commissioner of Internal Revenue. Any 
manufacturer of filled cheese who fails to comply with the provisions of this section, or with the regulations herein authorized, shall be deemed guilty of a misdemeanor, and upon conviction thereof shall be fined not less than five hundred nor more than one thousand dollars.

SEC. 6. That filled cheese shall be packed by the manufacturers in wooden packages only, not before used for that purpose, and marked, stamped, and branded with the words "filled cheese," in black-faced letters not less than two inches in length, in a circle in the center of the top and bottom of the cheese; and in black-faced letters of not less than two inches in length in line from the top to the bottom of the cheese, on the side in four places equidistant from each other; and the package containing such cheese shall be marked in the same manner and in the same number of places, and in the same description of letters as above provided for the marking of the cheese; and all sales or consignments made by manufacturers of filled cheese to wholesale dealers in filled cheese or to exporters of filled cheese shall be in original stamped packages. Retail dealers in filled cheese shall sell only from original stamped packages, and shall pack the filled cheese, when sold, in suitable wooden or paper packages which shall be marked and branded in accordance with the rules and regulations to be prescribed by the Commissioner of Internal Revenue, with the approval of the Secretary of the Treasury. Every person who knowingly sells or offers to sell, or delivers or offers to deliver, filled cheese in any other form than in new wooden or paper packages, marked and branded as hereinbefore provided and as above described, or who packs in any package or packages filled cheese in any manner contrary to law, or who falsely brands any package or affixes a stamp on any package denoting a less amount of tax than that required by law, shall upon conviction thereof be fined for each and every offense not less than fifty dollars and not more than five hundred dollars, or be imprisoned not less than thirty days nor more than one year.

SEc. 7. That all retail and wholesale dealers in filled cheese shall display in a conspicuous place in his or their salesroom a sign bearing the words, "Filled cheese sold here" in black-faced letters not less than six inches in length, upon a white ground, with the name and number of the revenue district in which his or their business is conducted; and any wholesale or retail dealer in filled cheese who fails or neglects to comply with the provisions of this section shall be deemed guilty of a misdemeanor, and shall on conviction thereof be fined for each and every offense not less than fifty dollars and not more than two hundred dollars. 
SEC. 8. That every manufacturer of filled cheese shall securely affix, by pasting on each package containing filled cheese manufactured by him, a label on which shall be printed, besides the number of the manufactory and the district and state in which it is situated, these words: "Notice.-The manufacturer of the filled cheese herein contained has complied with all the requirements of the law. Every person is cautioned not to use either this package again or the stamp thereon again, nor to remove the contents of this package without destroying said stamp, under the penalty provided by law in such cases." Every manufacturer of filled cheese who neglects to affix such label to any package containing filled cheese made by him or sold or offered for sale by or for him, and every person who removes any such label so affixed from any such package, shall be fined fifty dollars for each package in respect to which such offense is committed.

SEC. 9. That upon all filled cheese which shall be manufactured there shall be assessed and collected a tax of one cent per pound, to be paid by the manufacturer thereof; and any fractional part of a pound in a package shall be taxed as a pound. The tax levied by this section shall be represented by coupon stamps; and the provisions of existing laws governing the engraving, issue, sale, accountability, effacement, and destruction of stamps relating to tobacco and snuff, as far as applicable, are hereby made to apply to stamps provided for by this section.

SEC. 10. That whenever any manufacturer of filled cheese sells or removes for sale or consumption any filled cheese upon which the tax is required to be paid by stamps, without paying such tax, it shall be the duty of the Commissioner of Internal Revenue, within a period of not more than two years after such sale or removal, upon satisfactory proof, to estimate the amount of tax which has.been omitted to be paid and to make an assessment therefor and certify the same to the collector. The tax so assessed shall be in addition to the penalties imposed by law for such sale or removal.

SEC. 11. That all filled cheese, as herein defined, imported from foreign countries shall, in addition to any import duty imposed on the same, pay an internal revenue tax of eight cents per pound, such tax to be represented by coupon stamps; and such imported filled cheese and the packages containing the same shall be stamped, marked, and branded, as in the case of filled cheese manufactured in the United States.

SEC. 12. That any person who knowingly purchases or receives for sale any filled cheese which has not been branded or stamped 
according to law, or which is contained in packages not branded or marked according, to law, shall be liable to a penalty of fifty dollars for each such offense.

SEC. 13. That every person who knowingly purchases or receives for sale any filled cheese from any manufacturer or importer who has not paid the special tax herein provided for shall be liable, for each offense, to a penalty of one hundred dollars, and to a forfeiture of all articles so purchased or received, or of the full value thereof.

SEc. 14. That whenever any stamped package containing filled cheese is emptied it shall be the duty of the person in whose hands the same is to destroy the stamps thereon; and any person who willfully neglects or refuses so to do shall, for each such offense, be fined not exceeding fifty dollars or imprisoned not less than ten days nor more than six months.

SEc. 15. That the Commissioner of Internal Revenue is authorized to have applied scientific tests, and to decide whether any substances used in the manufacture of filled cheese contain ingredients deleterious to health. But in case of doubt or contest, his decision in this class of cases may be appealed from to a board hereby constituted for the purpose, and composed of the Surgeon-General of the Army, the Surgeon-General of the Navy, and the Secretary of Agriculture, and the decision of this board shall be final in the premises.

SEc. 16. That all packages of filled cheese subject to tax under this act that shall be found without stamps or marks as herein provided, and all filled cheese intended for human consumption which contains ingredients adjudged as hereinbefore provided to be deleterious to the public health, shall be forfeited to the United States.

SEc. 17. That all fines, penalties and forfeitures imposed by this act may be recovered in any court of competent jurisdiction.

SEc. 18. That the Commissioner of Internal Revenue, with the approval of the Secretary of the Treasury, shall make all needful regulations for the carrying into effect the provisions of this act.

SEc. 19. That this act shall go into effect on the ninetieth day after its passage, and all wooden packages containing ten or more pounds of filled cheese found on the premises of any dealer on and after the ninetieth day succeeding the date of the passage of this act, shall be deemed to be taxable under section nine of this act, and shall be taxed, and shall have affixed thereto the stamps, marks, and brands required by this act or by regulations made pursuant to this act; and for the purpose of securing the affixing of the stamps, marks, and brands required by this act, the filled cheese shall be regarded as 
having been manufactured and sold or removed from the manufactory for consumption or use on or after the day this act takes effect; and such stock on hand at the time of the taking effect of this act may be stamped, marked, and branded under special regulations of the Commissioner of Internal Revenue, approved by the Secretary of the Treasury; and the Commissioner of Internal Revenue may authorize the holder of such packages to mark and brand the same and to affix thereto the proper tax-paid stamps.

Approved June 6, 1896.

\section{THE NEW YORK LAW}

The law of the state of New York is typical of the state laws governing the manufacture, sale, and adulteration of dairy products. It forms Chapter I. of the Consolidated Laws; the latter being Chapter IX. of the Laws of 1909, passed February 17, 1909, and is as follows:

\section{ARTICLE III}

\section{Dairy Products.}

SEC. 30. Definitions.-The term "butter" when used in this article means the product of the dairy, usually known by that term, which is manufactured exclusively from pure, unadulterated milk or cream or both with or without salt or coloring matter; and the term "cheese" when used in this article, means the product of the dairy usually known by that term, which is manufactured exclusively from pure, unadulterated milk or cream, or both, and with or without coloring matter, salt, rennet, sage, olives, pimentos, walnuts, peanuts, tomatoes, celery salt or onions added thereto as a flavor. And provided further, that when manufactured by adding to the elemental product of the dairy, usually known by the term "cheese," and manufactured exclusively from pure unadulterated milk or cream or both, any pimentos, olives, walnuts, peanuts, celery salt, tomatoes, or onions, that the percentage of all such substances so added shall not exceed twenty-five per centum in bulk of the manufactured product.

The terms "oleomargarine," "butterine," "imitation of butter" or "imitation cheese" shall be construed to mean any article or substance in the semblance of butter or cheese not the usual product of the dairy and not made exclusively of pure or unadulterated milk or 
cream, or any such article or substance into which any oil, lard or fat not produced from milk or cream enters as a component part, or into which melted butter or butter in any condition or state, or any oil thereof has been introduced to take the place of cream. The term "adulterated milk" when so used means:

1. Milk containing more than eighty-eight and one-half per centum of water or fluids.

2. Milk containing less than eleven and one-half per centum of milk solids.

3. Milk containing less than three per centum of fats.

4. Milk drawn from cows within fifteen days before and five days after parturition.

5. Milk drawn from animals fed on distillery waste or any substance in a state of fermentation or putrefaction or on any unhealthy food.

6. Milk drawn from cows kept in a crowded or unhealthy condition.

7. Milk from which any part of the cream has been removed.

8. Milk which has been diluted with water or any other fluid, or to which has been added or into which has been introduced any foreign substance whatever.

All adulterated milk shall be deemed unclean, unhealthy, impure and unwholesome. The terms "pure milk" or "unadulterated milk," when used singly or together, mean sweet milk not adulterated, and the terms "pure cream" or "unadulterated cream," when used singly or together, mean cream taken from pure and unadulterated milk. The term "adulterated cream" when used shall mean cream containing less than eighteen per centum of milk fat or cream to which any substance whatsoever has been added.

SEc. 31. Care and feed of cows, and care and keeping of the produce from such cows.- No person shall keep cows, for the production of milk for market or for sale or exchange, or for manufacturing the milk or cream from the same into any article of food, in a crowded or unhealthy condition or in unhealthful or unsanitary surroundings and no person shall keep such cows or the product therefrom in such condition or surroundings or in such places as shall cause or tend to cause the produce from such cows to be in an unclean, unhealthful or diseased condition, if the produce from such cows is to be sold, offered or exposed for sale upon the markets for consumption or to be manufactured into any food product, nor shall such cows or the produce therefrom be handled or cared for by any person suffering with or 
affected by an infectious or contagious disease, nor shall any such cows be fed on any substance that is in a state of putrefaction or fermentation, or upon any food that is unhealthful or that produces or may produce impure, unhealthful, diseased or unwholesome milk. But this section shall not be construed to prohibit the feeding of ensilage. The Commissioner of Agriculture is hereby empowered to give such instruction and impart such information as in his judgment may be deemed best to produce a full observance of the provisions of this section.

Sec. 32. Prohibiting the sale of adulterated milk, imitation cream and regulating the sale of certified milk.-No person shall sell or exchange or offer or expose for sale or exchange, any unclean, impure, unhealthy, adulterated or unwholesome milk or any cream from the same, or any unclean, impure, unhealthy, adulterated, colored, or unwholesome cream, or sell or exchange, or offer or expose for sale or exchange, any substance in imitation or semblance of cream, which is not cream, nor shall he sell or exchange, or offer or expose for sale or exchange any such substance as and for cream, or sell or exchange, or offer or expose for sale or exchange any article of food made from such milk or cream or manufacture from any such milk or cream any article of food. No person shall sell or exchange, or offer or expose for sale or exchange, as and for certified milk, any milk which does not conform to the regulations prescribed by and bear the certification of a milk commission appointed by a county medical society organized under and chartered by the medical society of the state of New York and which has not been pronounced by such authority to be free from antisepties, added preservatives, and pathogenic bacteria, or bacteria in excessive numbers. All milk sold as certified milk shall be conspicuously marked with the name of the commission certifying it. Any person delivering milk to any butter or cheese factory, condensary, milk-gathering station or railway station to be shipped to any city, town or village shall be deemed to expose or offer the same for sale whether the said milk is delivered or consigned to himself or another. Each and every can thus delivered, shipped or consigned, if it be not pure milk, must bear a label or card upon which shall be stated the constituents or ingredients of the contents of the can.

SEC. 33. Regulations in regard to butter and cheese factories.-No person shall sell, supply or bring to be manufactured to any butter or cheese factory any milk diluted with water, or any unclean, impure, unhealthy, adulterated or unwholesome milk, or milk from which any of the cream has been taken, except pure skim milk to skim-cheese 
factories. No person shall sell, supply or bring to be manufactured to any butter or cheese factory any milk from which there has been kept back any part of the milk commonly known as strippings, or any milk that is sour, except pure skim milk to skim-cheese factories. The owner or proprietor or the person having charge of any butter or cheese factory, not buying all the milk used by him, shall not use for his own benefit, or allow any of his employees or any other person to use for his own benefit, any milk, cream, butter or cheese or any other product thereof, brought to such factory, without the consent of the owners of such milk or the products thereof. Every butter or cheese manufacturer not buying all the milk he uses, shall keep a correct account of all the milk daily received, of the number of packages of butter and cheese made each day, and the number of packages and aggregate weight of cheese and butter disposed of each day; which account shall be open to inspection to any person who delivers milk to such factory. Whenever manufacturers of butter or cheese purchase milk upon the basis of the amount of fat contained therein and use for ascertaining the amount of such fat what is known as the Babcock test, or whenever the proceeds of co-operative creameries and cheese factories are allotted on the basis of determinations of milk fat by the Babcock test, the bottles and pipettes used in such test shall before use be examined by the director of the New York agricultural experiment station. If such bottles are found to be properly constructed and graded so as to accurately show the amount of fat contained in milk, each of them shall be legibly and indelibly marked "S. B." No bottle shall be so marked except as herein provided or used in any such test by such manufacturers, unless so examined and marked. The acid used in making such test by such manufacturers shall be examined from time to time by competent chemists employed by the Commissioner of Agriculture and if found not to be of sufficient strength the use of such acid shall be prohibited. No person or persons receiving or purchasing milk or cream upon the basis of the amount of fat contained therein shall credit any patron or patrons delivering milk or cream thereto with a greater or less percentage or average percentage of fat than is actually contained in the milk or cream so delivered. The Commissioner. of Agriculture or persons employed by him for that purpose may at any time assist in making tests of milk received at a butter or cheese factory for the purpose of determining the efficiency of tests usually made at such factory. All persons using other than standard bottles or acid which is not of the required strength to accurately determine the amount of fats in milk or crediting any patron or patrons delivering milk or cream 
with a greater or less percentage or average percentage of fat than is actually contained in the milk or cream so delivered, shall be subject to the penalties prescribed by Section fifty-two of this article, and shall be guilty of a misdemeanor.

Sec. 34. Penalty for delivery of adulterated milk.-Any person, firm, association or corporation delivering any milk to any butter or cheese factory in violation of any of the provisions of this chapter shall forfeit and pay to the patrons, firm, association or corporation owning the milk delivered to such factory the sum of fifty dollars, to be recovered in a civil action by the person, firm, association or corporation entitled thereto.

SEc. 35. Inspection; how conducted.-When the Commissioner of Agriculture, an assistant commissioner, or any person or officer authorized by the commissioner, or by this chapter, to examine or inspect any product manufactured or offered for sale shall in discharge of his duties take samples of such product, he shall, before taking a sample, request the person delivering the milk or who has charge of it at the time of inspection, to thoroughly stir or mix the said milk before the sample is taken. If the person so in charge refuses to stir or mix the milk as requested, then the person so requesting shall himself so stir and mix the milk before taking the sample, and the defendant shall thereafter be precluded from introducing evidence to show that the milk so taken was not a fair sample of the milk delivered, sold, offered or exposed for sale by him. The person taking the sample of milk for analysis shall take duplicate samples thereof in the presence of at least one witness, and he shall in the presence of such witness seal both of such samples, and shall tender, and, if accepted, deliver at the time of taking one sample to the manufacturer or vender of such product, or to the person having custody of the same, with a statement in writing of the cause of the taking of the sample. In taking samples of milk for analysis at a creamery, factory, platform or other place where the same is delivered by the producer for manufacture, sale or shipment, or from a milk vender who produces the milk which he sells, with a view of prosecuting the producer of such milk for delivering, selling or offering for sale adulterated milk, the said Commissioner of Agriculture or assistant or his agent or agents shall within ten days thereafter, with the consent of the said producer, take a sample in a like manner of the mixed milk of the herd of cows from which the milk first sampled was drawn and shall deliver the duplicate sample to the said producer and shall cause the sample taken by himself or his agent to be analyzed. If the sample of milk last taken by the Commissioner of Agriculture or 
his agent or agents shall upon analysis prove to contain no higher percentage of milk solids, or no higher percentage of fat than the sample taken at the creamery, factory, platform or other place, then no action shall lie against the said producer for violation of subdivisions one, two, three, seven and eight of section thirty of this chapter. In taking a second sample as above set forth from the mixed milk of the herd, it shall be the duty of the Commissioner of Agriculture to have an assistant, agent or agents present during the entire time in which the said cattle are being milked to observe closely so as to be sure that the milk thus to be sampled is not adulterated and to see that it is thoroughly mixed so that the sample taken shall be a fair sample of the average quality of the mixed milk of the entire dairy or herd of cows of said producer. If, however, the said producer refuses to allow such examination of the milk produced by his dairy, then he shall be precluded from offering any evidence whatever tending to show that the milk delivered by him at the said creamery, factory, platform or other place was just as it came from the cow. If the said producer does permit such examination, the Commissioner of Agriculture shall, upon receiving application therefor, send to said producer a copy of the analysis of each of the samples of milk so taken and analyzed as above provided. If a sample of milk shall have been taken by the Commissioner of Agriculture or by his orders or directions from any dairy within this state and an analysis thereof has been made by the Commissioner or by his authority, any person who is or was buying milk from the said dairy at or subsequent to the time of such taking, may apply to the Commissioner of Agriculture for a copy of the analysis of the said sample of milk so taken and the Commissioner shall thereafter furnish the said applicant with such copy.

SEC. 36. Branded cans, jars or bottles not to be sold, re-marked or used without consent of owner.-No person shall hereafter without the consent of the owner or shipper, use, sell, dispose of, buy or traffic in any milk can, jar or bottle, or cream can, jar or bottle, belonging to any dealer or shipper of milk or cream residing in the state of New York or elsewhere, who may ship milk or cream to any city, town or place within this state, having the name or initials of the owner, dealer or shipper, stamped, marked or fastened on such can, jar or bottle, or wilfully mar, erase or change by re-marking or otherwise said name or initials of any such owner, dealer or shipper, so stamped, marked or fastened upon said can, jar or bottle. Nor shall any person without the consent of the owner use such can, jar or bottle, for any other purpose than for milk or cream: nor shall any person without the consent of 
the owner place in any such can, jar or bottle, any substance or product other than milk or cream.

SEc. 37. Regulations in regard to condensed milk.-No condensed milk shall be made or offered or exposed for sale or exchange unless manufactured from pure, clean, healthy, fresh, unadulterated and wholesome milk from which the cream has not been removed either wholly or in part, or unless the proportion of milk solids shall be in quantity the equivalent of eleven and one-half per centum of milk solids in crude milk, and of which solids twenty-five per centum shall be fats. No person shall manufacture, sell or offer for sale or exchange in hermetically sealed cans, any condensed milk unless put up in packages upon which shall be distinctly labeled or stamped the name of the person or corporation by whom made and the brand by which or under which it is made. When condensed milk shall be sold from cans or packages not hermetically sealed, the producer shall brand or label the original cans or packages with the name of the manufacturer of the milk contained therein.

SEC. 38. Manufacture and sale of imitation butter prohibited.-No person by himself, his agents or employees, shall produce or manufacture out of or from any animal fats or animal or vegetable oils not produced from unadulterated milk or cream from the same, the article known as oleomargarine or any article or product in imitation or semblance of natural butter produced from pure, unadulterated milk or cream of the same; or mix, compound with or add to milk, cream or butter any acids or other deleterious substance or any animal fats or animal or vegetable oils not produced from milk or cream, so as to produce any article or substance or any human food in imitation or in semblance of natural butter, nor sell, keep for sale or offer for sale any article, substance or compound, made, manufactured or produced in violation of the provisions of this section, whether such article, substance or compound shall be made or produced in this state or elsewhere. Any person manufacturing, selling, offering or exposing for sale any commodity or substance in imitation or semblance of butter, the product of the dairy, shall be deemed guilty of a violation of this chapter, whether he sells such commodity or substance as butter, oleomargarine or under any other name or designation whatsoever and irrespective of any representations he may make relative to such commodity or substance. Any dealer in any article or product, the manufacture or sale of which is prohibited by this section, who shall keep, store or display such article or product, with other merchandise or stock in his place of business, shall be deemed to have the same in his possession for sale. 
SEC. 39. Manufacture or mixing of animal fats with milk, cream or butter prohibited.-No person shall manufacture, mix or compound with or add to natural milk, cream or butter any animal fats or animal or vegetable oils, nor make or manufacture any òleaginous substance not produced from milk or cream, with intent to sell the same as butter or cheese made from unadulterated milk or cream or have.the same in his possession with such intent; nor shall any person solicit or take orders for the same or offer the same for sale, nor shall any such article or substance or compound so made or produced, be sold as and for butter or cheese, the product of the dairy. No person shall coat, powder or color with annatto or any coloring matter whatever, butterine or oleomargarine or any compound of the same or any product or manufacture made in whole or in part from animal fats or animal or vegetable oils not produced from unadulterated milk or cream by means of which such product, manufacture or compound shall resemble butter or cheese, the product of the dairy; nor shall he have the same in his possession with intent to sell the same nor shall he sell or offer to sell the same. No person by himself, his agents or employees, shall manufacture, sell, offer or expose for sale, butter that is produced by taking original packing stock or other butter or both and melting the same, so that the butter fat can be drawn off, then mixing the said butter fat with skimmed milk or milk or cream or other milk product and rechurning the said mixture, or that is produced by any similar process and is commonly known as boiled or process butter, unless he shall plainly brand or mark the package or tub or wrapper in which the same is put up in a conspicuous place with the words "renovated butter" or "process butter." If the same shall be put up, sold, offered or exposed for sale in prints or rolls, then the said prints or rolls shall be labeled plainly with printed letters in a conspicuous place on the wrapper with the words "renovated butter" or "process butter." If the same is packed in tubs or boxes or pails or other kind of a case or package the words "renovated butter" or "process butter" shall be printed on the top and side of the same in letters, at least, one inch in length, so as to be plainly seen by the purchaser. If such butter is exposed for sale, uncovered, not in a package or case, a placard containing the label so printed shall be attached to the mass of butter in such manner as to easily be seen and read by the purchaser. Every person selling, offering or exposing for sale at retail, "renovated butter" or "process butter," shall cause each parcel or package of such butter delivered to or for a customer to be wrapped in a light-colored paper on which shall be printed in black letters, not less than three-eighths inch square and in Gothic type, the 
words "renovated butter" or "process butter." No person shall sell, offer or expose for sale, any butter or other dairy products containing a preservative, but this shall not be construed to prohibit the use of salt in butter or cheese, or spirituous liquors in club or other fancy cheese or sugar in condensed milk. No person, firm, association or corporation shall induce or attempt to induce any person to violate any of the provisions of this chapter. Any person, firm, association or corporation selling, offering or advertising for sale any substance, preparation or matter for use in violation of the provisions of this chapter shall be guilty of a violation of this section.

SEc. 40. Prohibited articles not to be furnished for use.-No keeper or proprietor of any bakery, hotel, boarding-house, restaurant, saloon, lunch-counter or place of public entertainment, nor any person having charge thereof or employed thereat, nor any person furnishing board for any others than members of his own family, or for any employees where such board is furnished for a compensation or as part of the compensation of any such employee, shall keep, use or serve therein either as food for his guests, boarders, patrons, customers or employees or for cooking purposes any article or substance made in violation of the provisions of this article. Any keeper or proprietor of any hotel, boardinghouse, restaurant, saloon, lunch-counter or place of public entertainment who uses or serves therein for his guests any oleaginous substance as a substitute for butter, the manuiacture or sale of which is not prohibited by the agricultural law, shall print plainly and conspicuously on the bill-of-fare, if there is one, the words, "Oleomargarine Used Here" and shall post up conspicuously in different parts of each room where such meals are served, signs in places where they can be easily seen and read, which shall bear the words, "Oleomargarine Used Here" in letters at least two inches in length and so printed as to be easily read by guests or boarders.

SEC. 41. Coloring matter, dairy terms, size of package, labeling, penalties.-No person manufacturing with intent to sell any substance or article in imitation or semblance of butter or cheese not made exclusively from unadulterated milk or cream or both, with salt or rennet or both and with or withcut coloring matter or sage, but into which any animal, intestinal or offal fats, or any oils or fats or oleaginous substance of any kind not produced from pure, unadulterated milk or cream, or into which melted butter, or butter in any condition or state or any modification of the same, or lard or tallow shall be introduced, shall add thereto or combine therewith any annatto or compounds of the same, or any other substance or substances whatever, for the pur- 
pose or with the effect of imparting thereto a color resembling yellow, or any shade of yellow butter or cheese, nor introduce any such coloring matter or other substance into any of the articles of which the same is composed. And no person selling any oleaginous substance not made from pure milk or cream of the same as a substitute for butter shall sell, give away or deliver with such substance any coloring matters nor shall any person manufacturing, selling or offering for sale any such goods make or sell them under any brand, device or label bearing words indicative of cows or the product of the dairy or the names of breeds of cows or cattle, nor use terms indicative of processes in the dairy in making or preparing butter; no such substance shall hereafter be sold, offered or exposed for sale in this state except it be sold in packages containing not more than five pounds, such packages to be wrapped and sealed, the original seal of which shall be unbroken and upon which seal shall be plainly printed the name and address of the manufacturer of said oleomargarine, and the said packages shall be plainly and conspicuously labeled with the word "Oleomargarine" in Gothic or equally conspicuous letters at least three-eighths of an inch high. The word "Oleomargarine" in large prominent letters shall be stamped by indentation on each separate brick or portion of the substance itself before it is wrapped and sealed.

Any person violating any of the provisions of sections forty or fortyone of the agricultural law shall forfeit and pay a penalty to the people of the state of New York of not less than fifty dollars nor more than one hundred dollars for the first violation and not less than two hundred dollars nor more than five hundred dollars for the second and each subsequent violation. Whoever by himself or another violates any of the provisions of sections forty or forty-one of the agricultural law shall be guilty of a misdemeanor and upon conviction shall be punished by a fine of not less than one hundred dollars nor more than five hundred dollars or by imprisonment of not less than one month nor more than one year or by both such fine and imprisonment for the first offense and by not less than six months nor more than one year for the second offense.

SEc. 42. Coloring matter in food products; analysis by state board of health.-No person or persons shall manufacture, sell or expose for sale any poisonous coloring matter for the coloring of food products of any kind, nor shall any person or persons use any poisonous coloring matter manufactured, sold, offered or exposed for sale within this state; nor shall any person or persons sell, offer or expose for sale any food product containing such poisonous coloring matter. The state com- 
missioner of health shall cause samples of coloring matter that are exposed for sale upon the market for use in food products to be analyzed and report the results of such analysis to the legislature at the next session.

SEC. 43. Manufacture and sale of imitation cheese prohibited.-No person shall manufacture, deal in, sell, offer or expose for sale or exchange any article or substance, in the semblance of or in imitation of cheese made exclusively of unadulterated milk or cream, or both, into which any animal, intestinal or offal fats or oils, or melted butter or butter in any condition or state or modification of the same, or oleaginous substances of any kind not produced from unadulterated milk or cream, shall be introduced.

SEc. 44. When prohibitions do not apply to skim milk or skim cheese. -Except in the counties of New York and Kings, the prohibitions contained in this article against the sale of adulterated milk shall not apply to skim milk, which is clean, pure, healthy, wholesome and unadulterated, except by skimming, sold for use in the county in which it is produced or an adjoining county, if it is sold for and as skimmed milk. The prohibitions in this article against the sale of cheese made from unadulterated* milk or cream, shall not apply to pure skimcheese made from milk which is clean, pure, healthy, wholesome and unadulterated, except by skimming.

SEC. 45. Unclean receptacles and places for keeping milk; notice to violators of provisions.-No person, firm, association or corporation, producing, buying or receiving milk for the purpose of selling the same for consumption as such, or for manufacturing the same into butter, cheese, condensed milk, or other human food, shall keep the same in utensils, cans, vessels, rooms, or buildings that are unclean or have unsanitary surroundings or drainage or in any condition whatsoever that would tend to produce or promote conditions favorable to unhealthfulness or disease. The Commissioner of Agriculture shall notify all persons, firms, associations or corporations, violating this section, to clean said utensils, cans, vessels, rooms or buildings, or to so improve the sanitary conditions that the law will not be violated, and if such notice is complied within ten days' time, Sundays excepted, then no action shall lie for a violation of this section. Any person having charge of any milk-gathering station where milk is bought or received from the dairymen for the purpose of selling the same for consumption or shipping the same to market for consumption as human food before

*So in the original. 
taking such charge or operating or working as such agent or person in charge shall apply to the Commissioner of Agriculture for a license to so work or operate or have charge, and shall at the time of making such application, file with the Commissioner a statement under oath, setting forth the fact that he will not while having charge of or operating any such milk-gathering establishment or while employed therein adulterate or suffer or permit the adulteration of any such milk or any product thereof during the term for which he may be licensed. After the applicant shall have complied with the foregoing provisions of this section, the Commissioner of Agriculture upon being satisfied that the applicant is a person of good moral character and a qualified and proper person to so have charge of or operate any such milk-gathering station or establishment shall issue to said applicant a license, which shall qualify him to have charge of any such milk-gathering station or establishment for the period of two years from the date of such license. The person regularly doing the work of receiving, caring for and shipping the milk at any station or establishment, or in case more than one person is so employed then the foreman in charge of such works shall be deemed to be a person in charge of such station or establishment within the meaning and purposes of this section. Such a license certificate shall be kept at such station or establishment where the licensee is so employed and shall be open to the inspection of the representatives of the Department of Agriculture and the public. Any person having charge of any milk-gathering station or establishment as aforesaid shall keep a true and correct monthly record of the receipts of milk or other dairy products received at such station or establishment, and also a true and correct monthly record of all sales or shipments of milk, cream or other dairy products shipped or sold from such station or establishment, and shall also keep a true and correct monthly record of the amount of skim milk produced in such station or establishment and of the disposition of said skim milk. Such record shall be preserved at such station or establishment for at least two years after the same shall have been made and such records shall at all times be open to the inspection of the Commissioner of Agriculture, his assistants or agents. When cream is sold or shipped from any such station or establishment so selling or shipping milk for consumption as aforesaid, each original bottle or package of one quart or less of cream so shipped or sold shall bear a label securely attached to the side of such bottle or package on which shall be conspicuously printed the word "cream" in black letters of at least one-fourth of an inch in length or else the word "cream" shall be blown in the side of such bottle in plain raised letters 
of at least one-half an inch in length, and the top and side of each and every other original package or can containing cream or original crate or case containing bottles of cream so shipped or sold shall bear a label securely attached on which shall be conspicuously printed the word "cream" in black letters of at least one inch in length and also a plainly written or printed statement on the label stating from whom and what station the same is shipped and the name of the consignee and point of destination and the date on which the cream therein was produced by such separation or skimming. The shipment of each and every such original package of cream so shipped and not so labeled as herein required shall constitute a separate violation. When cream is so separated or skimmed from milk at any such station or establishment and the supply of milk on hand thereat at the time of the next regular daily shipment of milk therefrom, consisting of the total amount of milk in such shipment, together with that remaning on hand immediately after such shipment, is not thereby decreased or correspondingly less than the total quantity received during any period extending from some point of time before such skimming was done until the time of such shipment, together with the amount of milk on hand at the commencement of such period, and such decrease is not equal in amount to the quantity of milk that must have been used in so separating such cream in addition to the quantity otherwise there used or disposed of during such period, such fact is conclusive that skim milk or other foreign substance was added to such milk supply within such period and shall be presumptive evidence within the meaning of this section that the same was added to each can or vessel of milk in such shipment. When cream or skim milk is found to have been on the premises of any such station or establishment or is sold or shipped therefrom, such cream or skim milk so found or so sold or shipped therefrom shall be presumed to have been produced by separating or skimming at such station or establishment. In any action or proceeding relative to the adulteration of milk by removing cream therefrom or adding skim milk or other foreign substance thereto, it shall be presumed that when cream has been produced by so skimming or separating or butter has been manufactured, there was made at least five quarts of milk in the production of each quart of cream so produced and there was necessarily so produced thereby at least four quarts of skim milk to each quart of cream so produced, and that there was used at least nine quarts of milk in the production of each pound of butter so manufactured. If any such person so duly licensed shall thereafter refuse or neglect to keep and preserve full and complete records as herein required or shall refuse 
to exhibit such records to the Commissioner of Agriculture, his assistants or agents or shall violate any of the provisions of this section or any of the provisions of this chapter relative to milk or the products thereof he shall forfeit his license and shall be disqualified for a period of five years from being again licensed by the Commissioner of Agriculture.

SEc. 46. Unsanitary cans and receptacles condemned.-All cans, or receptacles used in the sale of milk, cream or curd for consumption, or in transporting or shipping the same to market or the delivery thereof to purchasers for consumption as human food, when found by the Commissioner of Agriculture or his assistants or agents to be in unfit condition to be so used by reason of being worn out, badly rusted, or with rusted inside surface, or unclean or unsanitary or in such condition that they can not be rendered clean and sanitary by washing, and will tend to produce or promote in milk, cream or curd when contained therein, bad flavors, unclean or unwholesome conditions favorable to unhealthfulness or disease, shall be condemned by the Commissioner of Agriculture or his assistants or agents. Every such can or receptacle when so condemned shall be marked by a stamp, impression or device, designed by the Commissioner of Agriculture, showing that it has been so condemned, and when so condemned shall not thereafter be used by any person for the purpose of so selling, transporting or shipping milk, cream or curd.

SEc. 47. Receptacles to be cleansed before returning; receptacles may be seized; evidence; violation; milk can inspectors.-Whenever any can or receptacle is used for transporting or conveying milk, cream or curd to market for the purpose of selling or furnishing the same for consumption as human food, which can or receptacle, when emptied, is returned or intended to be returned to the person so selling, furnishing or shipping such substance to be again thus used, or which is liable to continued use in so transporting, conveying, selling or shipping such substance as aforesaid, the consumer, dealer or consignee using, selling or receiving the milk, cream or curd from such can or receptacle, shall, before so returning such can or receptacle remove all substances foreign to milk therefrom, by rinsing with water or otherwise. When any such milk, cream or curd is sold within any city of this State or shipped into any such city, the fact of such shipment or sale shall be prima facie evidence that the same was so shipped or sold for consumption as human food. When any such can or receptacle is returned or delivered or shipped to any person or creamery so selling such substance within, or shipping the same into such city, it is deemed that such can 
or receptacle is liable to such continued use in so selling or shipping such substance therein for consumption as human food within the meaning and purposes of this section and section forty-six. No person shall place or suffer to be placed in any such can or receptacle any sweepings, refuse, dirt, litter, garbage, filth or any other animal or vegetable substance, nor shall any such consignee or other person through himself, his agent or employee, bring or deliver to any person or railroad or other conveyance any such can or receptacle for the purpose of such return, or any milk, cream or curd can or receptacle for the purpose of delivery or shipment to any person or creamery engaged in so selling or shipping such substances for consumption as human food, which can or receptacle contains such foreign substance or which has not been rinsed as herein provided. The word "curd" as used in this section and section forty-six applies to the substance otherwise known as "pot cheese" or "cottage cheese." Whenever any such can or receptacle is used, returned, delivered or shipped in violation of this section, or of section forty-six of this chapter, every such use, return, delivery or shipment of each such can or receptacle shall be deemed a separate violation thereof. Such cans or receptacles so used, returned, delivered or shipped in violation of this section or of section forty-six may be seized by the Commissioner of Agriculture, his assistants or agents and held as evidence of such violation. For the proper enforcement of this section and section forty-six, the Commissioner of Agriculture may appoint two milk can inspectors to be stationed chiefly in the city of New York who shall receive the usual compensation of other agents of the Department of Agriculture.

SEc. 48. Manufacturer's brand of cheese.-Every manufacturer of whole-milk cheese may put a brand or label upon such cheese indicating "whole-milk cheese" and the date of the month and year when made; and no person shall use such a brand or label upon any cheese made from milk from which any of the cream has been taken. The Commissioner of Agriculture shall procure and issue to the cheese manufacturers of the State, on proper application therefor, and under such regulations as to the custody and use thereof as he may prescribe, a uniform stencil brand or labels bearing a suitable device or motto, and the words, "New York State whole-milk cheese." Every such brand or label shall be used upon the outside of the cheese and shall bear a different number for each separate factory. The Commissioner shall keep a book, in which shall be registered the name, location and number of each manufactory using the brands or labels, and the name or names of the persons at each manufactory authorized to use the 
same. No such brand or labels shall be used upon any other than wholemilk cheese or packages containing the same.

SEc. 49. Use of false brand prohibited.-No person shall offer, sell, or expose for sale, in any package, butter or cheese which is falsely branded or labeled.

SEc. 50. County trade marks.-At a regular or special meeting of a county dairymen's association in any county of the State there may be adopted a county trade mark, by a majority of the members present and voting, to be used as a trade mark by a person manufacturing pure unadulterated butter or full-cream cheese in such county. The secretary of the association shall forthwith send to the Commissioner of Agriculture a copy of such trade mark, which copy he shall place on file in his office, noting thereupon the day and hour he received the same. But one county trade mark for butter and for cheese shall be placed on file for the same county. No association shall adopt any trade mark of any county already on file, or use that of any other county in the formation of a trade mark.

SEC. 51. Object and intent of this article.-This article and each section thereof are declared to be enacted to prevent deception in the sale of dairy products, and to preserve the public health, which is endangered by the manufacture, sale and use of the articles or substances herein regulated or prohibited.

SEC. 52. Penalties.-Every person violating any of the provisions of this chapter, shall forfeit to the people of the State of New York the sum of not less than fifty dollars nor more than one hundred dollars for the first violation and not less than one hundred dollars nor more than two hundred dollars for the second and each subsequent violation. When such violation consists of the manufacture or production of any prohibited article, each day during which or any part of which such manufacture or production is carried on or continued, shall be deemed a separate violation. When the violation consists of the sale, or the offering or exposing for sale or exchange of any prohibited article or substance, the sale of each one of several packages shall constitute a separate violation, and each day on which any such article or substance is offered or exposed for sale or exchange shall constitute a separate violation. When the use of any such article or substance is prohibited, each day during which or any part of which said article or substance is so used or furnished for use, shall constitute a separate violation, and the furnishing of the same for use to each person to whom the same may be furnished shall constitute a separate violation. Whoever by himself or another violates any of the provisions of articles three, four, 
six, eight and nine or sections three hundred fourteen and three hundred fifteen of this chapter or of sections one hundred six, one hundred seven and one hundred eight of this chapter shall be guilty of a misdemeanor, and upon conviction shall be punished by a fine of not less than fifty dollars, nor more than two hundred dollars, or by imprisonment of not less than one month nor more than six months or by both such fine and imprisonment, for the first offense; and by six months' imprisonment for the second offense.

SEC. 53. Butterine and similar products not to be purchased by certain institutions. - No money appropriated by law for maintenance and support in whole or in part of a State institution; nor money received by a charitable, benevolent, penal or reformatory institution from the State or from a county, city or town thereof appropriated by such county, city or town, for the maintenance or support in whole or in part of such institution; nor money belonging to or used for the maintenance or support of such institution, shall be expended for the purchase of, or in payment for, butterine, oleomargarine, lard, cheese, or articles or products in imitation or semblance of natural butter or cheese produced from pure unadulterated milk or cream from the same, which articles or products have been rendered or manufactured in whole or in part from animal fats, or animal or vegetable oils not produced from unadulterated milk or cream from the same.

SEC. 54. Purchase, sale and use of butterine and similar products prohibited in certain institutions.-No officer, manager, superintendent or agent of an institution mentioned in section fifty-three of this chapter, shall purchase for the use of such institution articles or products, for the purchase of which the money appropriated by law, or by a county, city or town, is forbidden to be used by section fifty-three of this chapter, and no person shall sell to, or for the use of such institution, such articles or products. Nor shall such articles or products be used as articles of food or for cooking purposes in such institutions within this State. 


\section{REFERENCES TO AGRICULTURAL EXPERIMENT STATION REPORTS AND BULLETINS}

The following references will aid the student who desires to make a more thorough study of the subjects discussed. They are taken wholly from the literature of American agricultural investigations, and include only some of the more important articles. The Experiment Station Record and the Handbook of Experiment Station Work, both issued by the U. S. Department of Agriculture, contain many condensed results of dairy investigation.

\section{Chapter I}

Studies in Milk Secretion. Indiana Bull. No. 24, pp. 13-16.

How is Milk Formed? Nevada Bull. No. 16, pp. 4-5.

Elaboration of Milk. Ontario Rept. for 1893, pp. 165-166.

Dividing Milkings. Indiana Bull. No. 24, pp. 10-13.

Milking Two and Three Times per Day. Vermont Rept. for 1890, pp. 90-96.

\section{Chapter II}

The Composition of Milk. Ontario Bull. No. 39.

The Mineral Ingredients of Milk. Maine Rept. for 1890, Part II., pp. 52-57.

The Constitution of Milk, and Some of the Conditions Which Affect the Separation of Cream. Wisconsin Bull. No. 18.

Conditions Affecting the Consistency of Milk. Wisconsin Rept. for 1896 , pp. $73-80$.

Chemistry of Dairy Products. Ontario Rept. for 1890, pp. 237-241.

Milk Analysis. Connecticut Rept. for 1886, pp. 119-130.

Investigations Relating to the Composition of Milk. Wisconsin Rept. for 1890, pp. 114-119.

Fibrin in Milk. Wisconsin Rept. for 1893, pp. 143-145.

Relation of Fat and Casein in Milk. Vermont Rept. for 1890, pp. 97-100.

The Composition, Creaming and Churning of Colostrum. Vermont Rept. for 1891, pp. 104-108.

The Fat Globules of Milk. New York Rept. for 1885, pp. 266275; Wisconsin Rept. for 1894, pp. 223-239; Ontario Rept. for 1885, pp. $127-130$. 
The Effects of Feed Upon the Quality of Milk. Iowa Bull. No. 14, pp. 123-142; New Hampshire Rept. for 1893, pp. 138-155, and Bull. No. 9; Bull. No. 16; Bull. No. 18; Bull. No. 20.

Tests of Several Breeds of Dairy Cows. A Study of Dairy Products. Maine Rept. for 1889, pp. 106-134. The test is continued in Rept. for 1890, Part II., pp. 17-42. Series.

Corn Silage for Milch Cows. New York (State) Bull. No. 97, New

Investigations of the Several Breeds of Dairy Cattle. New York (State) Reports for 1891, 1892, 1893, 1894.

On the Effects of Feeding Fat to Cows. New York (Cornell) Bull. No. 92.

Variations in Milk. nlinois Bull. No. 17, pp. 9-16, and Bull. No. 24.

The Influence of Advancing Lactation upon the Production of Butter and Cheese. New York (State) Rept. for 1891, pp. 369-389.

Effects of Drouth upon Milk Production. New York (State) Bull. No. 105. New Series.

Variations in Milk. Vermont Rept. for 1891, pp. 61-74.

Variations in Quantity and Quality of Milk. Vermont Rept. for 1892, pp. 90-119, and Bull. No. 38.

The Composition of Milk as Affected by Methods of Milking. Wisconsin Rept. for 1889, pp. 44, 51, 61 .

\section{Chapter IV}

Milk Tests. (Short, Lactoscope). Illinois Bull. No. 9, pp. 293-302.

Investigation of Milk Tests. (Short, Parsons, Failyer and Willard, Cochran, Patrick), Illinois Bull. No. 10.

Milk Tests: Methods of Testing Milk. (Patrick, Babcock, Beimling, Gravimetric.) Illinois Bull. No. 14, pp. 462-467.

Methods of Testing Milk. (Short, Patrick, Cochran, Babcock, Beimling.) West Virginia Bull. No. 13, pp. 41-63.

Testing Milk. (Short, Patrick, Cochran, Babcock, Soxhlet, Beimling.) West Virginia Report for 1890, pp. 68-88.

A New Volumetric Method for the Estimation of Fat in Milk, Skimmed Milk, Buttermilk and Cream. (Parsons.) New Hampshire Report for 1888 , pp. 69-83. 184.

Babcock and Beimling Tests. Ontario Report for 1891, pp. 183-

Simple Methods of Determining Milk Fat. (Short, Cochran, Gravimetric.) Pennsylvania Bull. No. 12. 
Comparative Test of Machines and Methods for the Determination of Fat in Milk. (Short, Beimling, Patrick, Babcock, Gravimetric.) Mississippi Bull. No. 15, pp. 5-16.

A Description of Cochran's Method for the Determination of Fat in Milk, for the Use of Dairymen. New York (Cornell) Bull. No. 17.

Iowa Station Milk Test. (Patrick.) Iowa Bull. No. 8, pp. 295316 ; No. 9 , p. 355 ; No. 11 , pp. 484-487.

A New Method for Determining the Amount of Butter Fat in Milk. Mississippi Bull. No. 21, pp. 17-19.

A New Milk Test. (Beimling.) Vermont Bull. No. 21.

The Lactanalyt, A New Milk Tester. Vermont Report for 1894, pp. 161-162.

A New Method of Milk Analysis (Short) for the Use of Dairymen, and a Comparison of Its Results with Those Obtained by the Churn. Kansas Report for 1888, pp. 149-164.

A Method for the Determination of Fat in Milk and Cream. (Parsons.) New York (State) Bull. No. 19. New Series.

Feser's Lactoscope and Fjord's Centrifugal Controller, described in Ontario Report for 1885, pp. 207-208.

Testing Milk at Creameries. (Short.) Vermont Bull. No. 16.

The Schoch and Bolender Test Churn. Wisconsin Report for 1884, pp. 23-25.

Description of the Test Churn. Ontario Report for 1885, p. 201.

The Oil Test for Cream. Wisconsin Búll. No. 12.

A New Method for Determining Fat in Milk. (Short.) Wisconsin Bull. No. 16 and Report for 1888, pp. 124-136.

The Babcock Milk Test was first described in Wisconsin Bull. No. 24 and Report for 1890 , pp. 98-113. Improvements and modifications are discussed in Bull. No. 31, pp. 3-16; Bull. No. 36, pp. 3-20; Bull. No. 52, Report for 1892, pp. 219-244; Report for 1893, pp. 116121. Compared with the Gravimetric Method in Report for 1896, pp. 138-143.

Elsewhere, the method has been described in Pennsylvania Bull. No. 33; Washington Bull. No. 18; New York (Cornell) Bull. No. 29, pp. 77-80; Colorado Bull. No. 20, pp. 3-10; North Carolina Bull. No. 113, pp. 101-111; West Virginia Bull. No. 13, pp. 52-57; Ontario Bull. No. 61; Bull. No. 93, pp. 5-6; Connecticut Bull. No. 106, pp. 2-9; Bull. No. 108, pp. 5-11; Bull. No. 117, Report for 1894, pp. 209-244; Maine Bull. No. 3, Second Series; Bull. No. 4, Report for 1891, Part II., pp. 71-80; Michigan Bull. No. 127; Illinois Bull. No. 27; North Dakota Bull. No. 22; Pennsylvania Report for 1895, Part II., pp. 
90-100; Nevada Bull. No. 16, pp. 41-51; Mississippi Bull. No. 15, pp. 7-14.

Milk Sampling. Delaware Bull. No. 31.

Composite Milk Samples Tested for Butter Fat. Illinois Bull. No. 16, pp. 504-515. Continued in Büll. No. 18, pp. 27-28.

The Composite Sample. Preservatives for Keeping Milk-Samples for Testing. Iowa Bull. No. 11, pp. 482-484.

Composite Samples at the Creamery-Chromate Preservatives. Iowa Bull. No. 22 , pp. 836-844.

Detection of Adulterations in Milk. Wisconsin Bull. No. 31, pp. 17-27; Bull. No. 36, pp. 21-31, and Report for 1892, pp. 245-257. Ontario Bull: No. 93, pp. 3-5; Vermont Newspaper Bull. No. 4.

Lactometer and Milk Test for Examining Milk. Minnesota Bull. No. 27 , pp. 55-56.

The Relation between Specific Gravity and Solids of Milk. Wisconsin Report for 1895, pp. 120-126.

The Estimation of the Total Solids in Milk from the Per Cent of Fat, and the Specific Gravity of the Milk. Wisconsin Report for 1891, pp. 292-307, and Report for 1893, p. 142.

The Lactometer and Fat Test for Cheese and Condensed Milk Factories. Maine Bull, No. 4, New Series, pp. 6-10.

\section{Chapter V}

Dairy Bacteriology, U. S. Dept. Agr., Office of Expt. Stations. Bull. No. 25.

The Fermentations of Milk. U. S. Dept. Agr. Expt. Stations. Bull. No. 9.

Souring of Milk. U. S. Dept. Agr. Farmers' Bull. No. 29.

Milk Fermentations and Their Relations to Dairying. U. S. Dept. Agr. Farmers' Bull. No. 9.

The Isolation of Rennet from Bacteria Cultures. Connecticut (Storrs) Report for 1892, pp. 106-126.

The Sources of Bacterial Infection, and the Relation of the Same to the Keeping Quality of Milk. Wisconsin Report for 1894, pp. 150-165.

Cleanliness in Handling Milk; Bacteriological Considerations. Nurth Dakota Bull. No. 21.

A Microccus of Bitter Milk. Connecticut (Storrs) Report for 1891, pp. 158-162.

Pasteurization of Milk and Cream for Direct Consumption. Wisconsin Bull. No. 44. 
Ropiness in Milk and Cream. Cornell Univ. Expt. Sta. Bull. No. 165. Bacteriology of Cheese. Wisconsin Report for 1903, pp. 226-230.

Action of Acid Producing Bacteria on Casein. Wisconsin Rept. for 1904, pp. 169-171.

Notes on Pasteurization of Milk and Cream. Wisconsin Rept. for 1895 , pp. 158-173.

On the Restoration of the Consistency of Pasteurized Milk and Cream. Wisconsin Rept. for 1896, pp. 81-94, and Bull. No. 54.

A Preliminary Bulletin on Pasteurization of Milk. Michigan Bull. No. 134 .

Preservation of Cream for Market. Maine Bull. No. 23, New Series.

\section{CHAP'TER VII}

Aëration and Aërators. New York (Cornell) Bull. No. 39, pp. 90-94.

Aëration of Milk. Vermont Rept. for 1892, pp. 123-128.

Concerning the Aëration of Milk. Indiana Bull. No. 44, pp. 37-39.

Town and City Milk Supply. U. S. Dept. Agr. Farmers' Bull. No. 42 , pp. $23-28$.

Variations in Fat of Milk Served to Customers in Dipping from Cans. New York (Cornell) Bull. No. 20, pp. 68-71.

Variations in the Fat of Milk. Ontario Bull. No. 46.

Relative Absorption of Odors in Warm and Cold Milk. Wisconsin Rept. for 1898, pp. 104-109.

The Efficiency of a Continuous Pasteurizer. New York (State) Bull. No. 172.

The Detection of Taints in Pasteurized Milk. Wisconsin Rept. for 1905, pp. 222-226.

Standardization of Milk and Cream. Illinois Bull. Nos. 74 and 75.

\section{Chapter IX}

Cream Raising by Dilution. New York (Cornell) Bull. No. 20, pp. 61-67; Bull. No. 29, pp. 65-71; Bull. No. 39, pp. 77-85; Illinois Bull. No. 12, pp. 376-377; Bull. No. 18, pp. 30-32; Vermont Rept. for 1890, pp. 100-107.; Vermont Rept. for 1898, pp. 365-366.

Other Methods of Setting Milk. Minnesota Bull. No. 19, pp. 1119; Iowa Bull. No. 25, pp. 39-40; Indiana Bull. No. 44, pp. 23-37; Wisconsin Rept. for 1884, pp. 17-22; Rept. for 1893, pp. 147-150; Bull. No. 7, pp. 9-13; Bull. No. 29; Canada, Central Experimental Farm 
Rept. for 1891, pp. 89-104; Rept. for 1892, pp. 71-74; Ontario Rept. for 1894, pp. 142-144; Vermont Rept. for 1891, pp. 100-101; Maine Bull. No. 5, Second Series; Utah Bull. No. 42.

The Viscosity of Milk. New York (State) Rept. for 1886, pp. 323-330.

The Constitution of Milk and Some of the Conditions Which Affect the Separation of Cream. Wisconsin Bull. No. 18.

The Centrifugal Separation of Casein and Insoluble Phosphates from Milk. Wisconsin Rept. for 1895, pp. 93-99.

Tests of Cream Separators. Delaware Bull. No. 17, and Rept. for 1892, pp. 110-122; Iowa Bull. No. 25, pp. 32-38; New Hampshire Rept. for 1893, pp. 36-45, and Bull. No. 70; New York (Cornell), Bull. No. 66 and 105; North Carolina Bull. No. 114; Pennsylvania Rept. for 1892, Part II., pp. 51-79, and Bull. No. 20; Bull. No. 27; Rept. for 1894, pp. 13-35; South Dakota Bull. No. 39; Vermont Bull. No. 27 and Rept. for 1892, pp. 136-143; Rept. for 1893, pp. 92-100; Rept. for 1894, pp. 151-160; Wisconsin Rept. for 1895, pp. 151-157, and Bull. No. 46.

\section{Chapters $\mathrm{X}, \mathrm{XI}, \mathrm{XII}$}

Experiments with Boyd's Vat and Starter. Ontario Rept. for 1891, pp. $178-179$. 14-41.

Bacteria in the Dairy. Connecticut (Storrs) Rept. for 1895, pp.

The Use of Bacterial Culture Starters in Butter Making, with Especial Reference to the Conn Culture (B. 41). Wisconsin Rept. for 1895, pp. 174-231; published, in part, in Bull. No. 48.

An Acid Test of Cream. Illinois Bull. No. 32, and Bull. No. 33, pp. 399-400.

The Alkaline Tablet Test of Acidity in Milk or Cream. Wisconsin Bull. No. 52, pp. 8-16.

Sweet versus Sour Cream Butter. Iowa Bull. No. 8, pp. 317-320; Bull. No. 11, pp. 481-482; Bull. No. 18, pp. 478-487; Bull. No. 21, pp. 788-791; Illinois Bull. No. 9, pp. 301-302; Texas Bull. No. 11, pp. 15-16; Ontario Rept. for 1891, pp. 179-181; West Virginia Rept. for 1890 , pp. $48-66$.

Creamery Studies of Methods and Machinery. A Comparison of the Sour Cream, Sweet Cream and Butter Extractor Processes. Delaware Rept. for 1890, pp. 17-23, and pp. 129-149; also Bull. No. 9.

Our Experience with Extractor Butter. Ontario Rept. for 1893, pp. 170-171. 
A Study in Churning. Iowa Bull. No. 22, pp. 819-832.

Churning Experiments. Vermont Rept. for 1893, pp. 100-106.

Butter Tests. New York (State) Rept. for 1884, pp. 334-347, and Rept. for 1885, pp. 275-292.

The Effect of Succulent Food upon the Churnability of the Fat in Milk. Vermont Rept. for 1890, pp. 70-74.

Butter Making. Ontario Rept. for 1889, pp. 161-163; Bull. No. 48.

Canada Central Experimental Farm Dairy. Bull. No. 3.

Washing and Salting Butter. Minnesota Bull. No. 7, pp. 34-42.

Influence of Conditions in Churning on Water in Butter. Iowa Bull. No. 52 .

Estimating Water in Butter by Overrun in Churning. Wisconsin Rept. for 1905, pp. 186-189.

The Relation of Acid Fermentation to Butter Flavor and Aroma. Iowa Bull. No. 40.

Commercial Butter Cultures. Pennsylvania Bull. No. 44.

Heated Milk for Butter Making. Pennsylvania Bull. No. 45.

Ripening Cream. Connecticut (Storrs) Bull. No. 21.

Pasteurization of Milk for Butter Making. Ontario Bull. No. 117.

Pasteurization as Applied to Butter Making. Wisconsin Rept. for 1903, pp. 167-176.

Perpetuation of Pure Cultures for Butter Starters. Oregon Bull. No. 83.

Use of Starters in Butter Making. Iowa Bull. No. 103.

Influence of Acidity of Cream in Flavor of Butter. U. S. Dept. Agr., Bureau Animal Industry, Bull. No. 114.

The Cause of Mottled Butter. Maryland Bull. No. 64 .

Effect of Salt on Water in Butter. Wisconsin Rept. for 1899, pp. 97-107.

White Spots in Butter. Wisconsin Rept. for 1899, pp. 118-120.

Effect of Feed on Quality of Butter. Vermont Rept. for 1901, pp. $375-377$.

Water in Butter. Ontario Rept. for 1902, pp. 38-39.

Keeping Quality of Butter. Iowa Bull. No. 71.

Moisture Content of Butter. Iowa Bull. No. 76.

Keeping Quality of Butter. U. S. Dept. Agr., Bureau of Animal Industry, Bull. No. 57.

Relation of Proteids to Mottled Butter. New York (State) Buil. No. 263.

Butter Preservatives. Ontario Bull. No. 145. 
Manufacture and Storage of Butter. U.S. Dept. Agr., Bureau Animal Industry, Bull. Nos. 84 and 89.

Comparison of Aniline and Annatto Butter Colors. Wisconsin Bull. No. 152.

A Study of Moisture in Butter. Iowa Bull. No. 101:

Paraffining Butter Tubs. U.S. Dept. Agr., Bureau Animal Industry, Bull. No. 130.

Factors Influencing the Composition of Butter. Illinois Bulls. Nos. 137, 138 and 139.

Keeping Quality of Butter. Michigan Tech. Bulls. Nos. 1 and 2.

\section{Chapters XIII, XIV}

For references to aëration, see Chapter VII.

Points of Attention for the Patrons of Cheese Factories and Creameries. Ontario Bull. No. 2.

Milk for Cheese Making. Ontario Bull. No. 49; Bull. No. 28; Bull. No. 94; Canada Central Experimental Farm Dairy. Bull. No. 1.

Pure Lactic Culture of Bacteria in Cheese Making. Wisconsin Rept. for 1896 , pp. 112-126.

Rennet Extracts of Commerce. Iowa Bull. No. 22, pp. 845-851.

Losses in Cheese Making. Vermont Rept. for 1891, pp. 95-100.

The Effect of Salt upon Cheese. Wisconsin Rept. for 1894, pp. $220-222$.

The Effect of Aëration on the Flavor of Tainted Curds in Cheese Making. The Influence of Acid on the Texture of Cheese. The Hot Iron Test. Experiments in Ripening the Milk before Setting. Wisconsin Rept. for 1895, pp. 127-138.

Experiments in the Manuiacture of Cheese. New York (State) Repts. for 1891, p. $216 ; 1892$, p. 295; 1893, p. 239; 1894, p. 263.

Hints to Cheese Makers. Iowa Bull. No. 19, pp. 627-631.

Investigations in Cheese Making. Iowa Bull. No. 21, pp. 735-767.

Experiments in Cheese Making. Minnesota Bull. No. 19, pp. 20-25.

Experiments in the Manufacture of Cheese. Wisconsin Rept. for 1894, pp. 131-149.

The Relation between Milk Solids and the Yield of Cheese. Wisconsin Rept. for 1895, pp. 100-119.

Notes for Cheese Makers for May. Ontario Bull. No. 40; for July, Bull. No. 43; for August, Bull. No. 44; for October, Bull. No. 47. Rept. for 1889 , pp. 163-179.

Notes for Cheese Makers for May. Canada Central Experimental 
Farm, Dairy Bull. No. 2; Special Dairy Bulletins for July, August, October and June.

Articles on Spring, Summer and Fall Cheese in Ontario Rept. for 1893, pp. 167-170.

Gas-producing Bacteria, and the Relation of the Same to Cheese. Wisconsin Rept. for 1895.

The Rise and Fall of Bacteria in Cheddar Cheese. Wisconsin Rept. for 1896, pp. 95-111.

An Aromatic Bacillus of Cheese. Iowa Bull. No. 21, pp. 792-796.

Changes During Cheese Ripening. Iowa Bull. No. 24, pp. 969-984.

Rusty Cans and Effect on Milk for Cheese Making. Wisconsin Bull. No. 162.

Propagation of Pure Starters in Cheese Making. Wisconsin Bull. No. 181.

Moisture Supply in Cheese Curing Rooms. Wisconsin Rept. for 1896, pp. 156-163.

Experiments upon the Curing of Cheese. Cornell University Agr. Exp. Sta. Rept. for 1880 , pp. 9-27.

Effect of Rennet Extract in Curdling Milk. Wisconsin Rept. for 1898, pp. 31-34.

The Action of Rennet in Watered Milk. Wisconsin Rept. for 1898, pp. 35-36.

The Effect of Salt on Rennet Action. Wisconsin Rept. for 1898, pp. 37-41.

Action of Ferments in Ripening Cheese. Wisconsin Rept. for 1899, pp. 57-174.

Effect of Digesting Bacteria on Cheese Solids. Wisconsin Rept. for 1899.

Coating Cheese with Paraffin. Wisconsin Rept. for 1899, pp. 153-154.

Influence of Cold Curing on Quality of Cheese. Wisconsin Rept. for 1901, pp. 136-161.

A Study of Enzymes in Cheese. New York (State) Bull. No. 203.

Conditions Affecting Weight Lost in Cheese Curing. New York (State) Bull. No. 207.

Salts Formed by Casein and Paracasein in Cheddar Cheese Making. New York (State) Bull. No. 214-219.

Ripening Cheese in Cold Storage. Ontario Bull. No. 121.

Influence of Temperature of $60^{\circ}$ on Flavor in Cold Cured Cheese. Wisconsin Rept. for 1902, pp. 165-183.

Cold Curing of Cheese. U. S. Dept. Agr., Bureau Animal Industry, Bull. No. 49 . 
Ripening Cheese. New York (State) Bulls. Nos. 233, 234, 236 and 237.

Shrinkage of Cold Cured Cheese. Wisconsin Bull. No. 101.

Experimental Work in Cheese Making. Wisconsin Rept. for 1903, pp. 188-192.

Investigations in Curing Cheese. Wisconsin Rept. for 1903, pp. 193-219.

Canning Cheese. Oregon Bull. No. 78.

Relations of Bacteria to Flavor of Cheddar Cheese. U. S. Dept. Agr., Bureau Animal Industry, Bull No. 62; Wisconsin Report for 1904.

Relation of Casein and Paracasein to Cheddar Cheese. New York (State) Bull. No. 261.

Cold Storage of Cheese. U. S. Dept. Agr., Bureau Animal Industry, Bull. No. 83.

Influence of Metals on Action of Rennet. Wisconsin Rept. for 1907, pp. 134-159.

An Automatic Cheese Press. Wisconsin Rept. for 1907.

Defects in Cheddar Cheese. New York (Cornell) Bull. No. 257.

The First Chemical Changes in Cheddar Cheese. New York (State) Tech. Bull. No. 4.

The Bacterial Flora of Cheddar Cheese. New York (State) Tech. Bull. No. 8.

\section{Chapter XV}

The Manufacture of Sweet Curd Cheese (Edam and Gouda). Minnesota Rept. for 1894, pp. 104-128, and Bull. No. 35.

Experiment Relating to the Manufacture of Edam and Gouda Cheese. New York (State) Rept. for 1893, pp. 244-269, and Bull. No: 56.

Albumin Cheese. Wisconsin Rept. for 1895, pp. 134-136.

Chemical Changes in Souring Milk in Relation to Cottage Cheese. New York (State) Bull. No. 245.

Soft Cheese Studies in Europe. U. S. Dept. Agr. Rept. for 1905, pp. 75-109.

Varieties of Cheese. U. S. Dept. Agr., Bureau Animal Industry, Bull. No. 105.

Care and Testing of Camembert Cheese. U. S. Dept. Agr., Bureau Animal Industry, Rept. for 1907, pp. 339-343.

Camembert Cheese Problems in United States. Connecticut (Storrs) Bull. No. 58.

Fancy Cheese for Farm and Factory. New York (Cornell) Bull. No. 270. 


\section{Chapter XVI}

The Manufacture of Ice Cream. Iowa Bull.-No. 123; Vermont Bull. No. 155 .

\section{Chapter XVII}

The Manufacture of Milk Sugar (Report of Chemist). Delaware Report for 1891, pp. 104-108.

The Hog as an Adjunct to the Dairy. Ontario Rept. for 1889, pp. 184-189.

Whey Butter. New York (Cornell) Bull. No. 85.

Sweet Skim Milk; Its Value as Food for Pigs and Calves. Wisconsin Bull. No. 1.

The Feeding Value of Whey. Wisconsin Bull. No. 27; Report for 1891 , pp. 38-48.

Feeding Waste Products of the Dairy. Wisconsin Report for 1886, pp. 21-25.

The Value of Creamery Separator Skim Milk for Swine Feeding. Wisconsin Report ior 1895, pp. 7-23.

Whey Butter of Swiss Cheese. Wisconsin Bull. No. 132.

Pasteurization and Inspection of Creamery By-products. Wisconsin Bull. No. 148.

\section{Chapter XVIII}

Building Creameries and Organization of Coöperative Creamery Companies. South Dakota Bull. No. 46.

Creameries for Texas; Plans and Specifications in Full for Creamery Outfit. Texas Bull. No. 5.

Coöperative Creameries. Minnesota Report for 1894, pp. 93-103, and Bull. No. 35.

The Establishment of Cheese Factories and Creameries. Special Bull. of the Central Canada Experimental Farm, Ottawa.

By-Laws, Rules and Regulations for Cheese Factories. Canada Central Experimental Farm Dairy Bull. No. 9.

Construction of Cheese Curing Rooms. Wisconsin Bull. No. 70.

Improvement of Cheese Curing Rooms. Ontario Dairy Div. Bull. No: 1.

Plans for a Cool Cheese Curing Room. Ontario Dairy Div. Bull. No. 7.

By-Laws, Rules and Regulations for Creameries on the CreamGathering Plan. Central Experimental Farm, Dairy Bull. No. 10. 


\section{Chapter XIX}

Statistics of the Dairy: U. S. Dept. Agr., Bureau of Animal Industry. Bull. No. 11.

Returns from the Ninth, Tenth, and Eleventh Censuses, Relating to the Production of Milk, Butter and Cheese on the Farm. U. S. Dept. Agr., Report of the Statistician, No. 113, pp. 115-118.

\section{General}

Facts About Milk. U. S. Dept. of Agr., Farmers' Bull. No. 42.

The Dairy Industry in Denmark. U. S. Dept. Agr., Bureau of Animal Industry, Bull. No. 5.

The Creamery Industry. Nevada Bull. No. 16.

Dairying. South Carolina Bull. No. 19.

Dairying in California. U. S. Dept. Agr., Bureau of Animal Industry, Bull. No. 14.

Dairy Farming in Washington. Washington Bull. No. 2, pp. 23-27.

The Dairy Industry in Nebraska, South Dakota, and North Dakota. U. S. Dept. Agr., Bureau of Animal Industry, Bull. No. 16. 



\section{INDEX}

Abnormal fermentations......... 113

Accumulator......... . . . . . . . 191

Accuracy of Babcock test glassware : 99

Acid, boracic................ 121

- butyric...............20, 23

- capric.................. 23

- caproic.................. 23

- caprylic................. 23

- citric.................. 26

- dioxystearic.............. . 23

- formation of lactic........... 198

- hydrochloric in ripening cream. 197

- lactic.......25, 116, 196, 208, 241

- lactic, determination of . . .205, 351

- lauric................... 23

- myristic................. 22

- oleic................... 22

- palmitic.................. 22

- salicylic................. 121

- stearic................. 22

- sulphuric................... 104

- tests................... 204

Acini.................... 3

Act, Filled Cheese......... . 345, 382

— oleomargarine ......... 344, 369

Adams paper-coil method........ 78

Adjustable, separator........... 191

Advanced registry of cows........ 45

Aëration of milk. . . . . . . . 138, 242

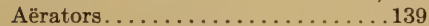

Aërometer, Soxhlet's............ 87

Agar.............. 127, 128, 131

- lactose.............. . . . 129

Agricultural Experiment Station

Bulletins.................404

Air drainage... . . . . . . . . . . . 327

Air space necessary in stables..... 153

Albumin................ 23, 24

Albuminoid fermentations........ 117

Albuminoids................. 23

Alcoholic fermentation.......... 25

Alderney cattle .............. 66
PAGE

Alexandra Jumbo separator . . .191, 192

Aliquot milk samples, Scovell...... 103

Alkali, decinormal......... 204, 351

$-\frac{\mathrm{m}}{\mathbf{1 0}} \ldots \ldots \ldots \ldots \ldots \ldots \ldots$. . . . . . . 351

- use of in cleaning............136

Alkaline tablets, Farrington's.... . .

$124,205,351$

Alpha B separator.............. 191

- Baby separator. . . . . . . . . . . 191

- discs.................... 187

- No. 1 separator. . . . . . . . . . . . 192

- separator................ 187

Alveoli...................... 3

American cheddar process. . . . . . . 249

- home-trade cheese. . . . . . . . . . 270

- Neufchatel cheese... . . . . . . . 273

Amphoteric reaction............ 108

Analysis, gravimetric.......... 77

Analyses of milk............. 17

Angularity, relation of to capacity . . 42

Angus, J. J., mentioned .......... 323

Aniline butter color.............231

Animal, effect of, on milk secretion. 14

- excrement, relation of bacteria

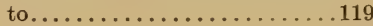

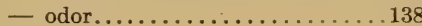

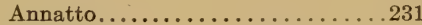

Antiseptics.................. 120

Apparatus bacteriological......... 126

- Fjord's control ............. 88

- pasteurizing................. 123

Arnold, L. B., quoted .......... 170

Arnold's separator.............. 191

Aromatics and stimulants....... 50

Artificial butter... . . . . . . . . . 344

— starter, preparation of . . . . . . 354

Asbestos method, Babcock....... 78

Ash...................... 25

Asses' milk............... 16, 36

Associated dairying.............340

Associations, cow-testing........ 47 


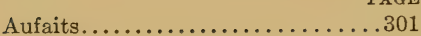

Autoclave... . . . . . . . . . . . . . 134

Avoiding flavors due to fcod....... 21

Ayrshire cattle... . . . . . . . . . . . 69

- milk.................. 33

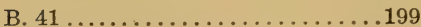

Babcock asbestos method........ 78

- centrifugal. ................ 94

- formula for total solids ..... . . 350

- glassware, calibration of . . . . . . 359

Babcock, S. M., mentioned .... 89, 91

—, S. M., quoted ...............

$14,17,147,170,171,236$

- test................. 91

— - acid for................ 104

- black specks in........... 105

- - bottle................. 95

- calibration of glassware.... 99

— - details of .............. 94

— - for butter............. . 99, 359

— - for cheese........... 99, 359

- - for cream ............. 97

- - for skim mik............ 98

- - glassware.............. 95

- - - accuracy of........... 99

- - cleaning.............107

— - reading the fat...........106

- - sampling milk............101

- temperature of reading fat

95,106

Baby separator................ 191

— - De Laval. . . . . . . . . . . . . . . 193

Bacillus.... . . . . . . . . . . . . 109

- acidi-lactici................ . 115

- No. 41.................. . . . 199

- prodigiosus................ 113

- tubercle.................. . 122

Bacteria.................... . . 109

- determination of in milk. . . . . 125

- presence of in milk .......... 111

- relation of to animal excrement.119

- - - dust to............. 119

- - to hay and dried forage... . 119

Bacteriological apparatus......... 126

- laboratory................. 126

- media.................. 127

Bad flavors in milk... . . . . . . . . 143

Balanced ration...............51

B. and W. test bottle..........996

Barber, quoted...... . . . . . . . . 200

Batch freezers. . . . . . . . . . . . . 307

Beimling, H. F., mentioned....... 92
PAGE

Beimling test................ 92

Belted cattle, Dutch..... . . . . . 74

Bernstein, Alexander, mentioned...324

Bichromate of potash........... 102

Biscuit ice cream........... . . 300

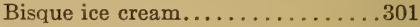

Bitter milk. . . . . . . . . . . . 113, 143

Bixa orellana.................231

Black specks in Babcock test. . . . . 105

Blended milk, Federal standard for. 365

Blood, relation of to milk secretion. . 11

"Bloody bread".................113

Board of Health lactometer . . . 80, 349

Boracic acid................ 121

Borden, Gail, Jr., mentioned . . . . . 316

Bottle, Babock test. . . . . . . . . . 95

Bottling milk. ............142; 162

Bouillon.................... 131

- nutrient................. . 127

Bowls, separator............. 179

Brands for cheese, State.......... 345

"Breaking" of butter. . . . . . . . . 216

Breed, relation of to milk production................ 52

- selection of .............. 52

- dairy.................. 63

Brie cheese................. . . 295

Brine freezers.................. 307

— salting butter............. 227

Browne, quoted.... . . . . . . . . . 22

Brown separator, medium......... 191

Brown Swiss cattle........... 73

Buildings, dairy . . . . . . . . . . 326

Bulletins, references to..........4404

Bull, management of ........... 57

— selection of .............. 55

Burette...................206

Butter accumulator.............. 191

— and cheese factories combined... 335

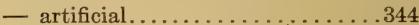

- Babcock test for . . . . . . . . . . . 359

— "breaking" of ..............2. 216

- brine salting.............227

- color of. .................231

- colors...................231

- color, aniline ..............231

- composition of..............229

— "factory". . . . . . . . . . . . . 346

- factories................... . . 326

- - arrangement of ............327

- construction of ..........330

— fat, Federal standard for. . . . . 366

- Federal standard for.......... 366 
PAGE

232

- flavor of...................230

- granules, size of............221

- imitation creamery........... . 346

— marketing...............227

- moisture-test, Cornell . . . . . . . . 359

- mottled and streaked.........226

- packages.................228

— packing...................227

— prints...................229

— "process".................. . .346

— quality of..............229

- rancid................ 20

- relation of lactic acid to keeping quality..................207

- relation of wash water to flavor. 223

- relation of wash water to texture..................222

- renovated......................... 346

— salting..................224

— score card for..............232

— "standing up" quality ......... 196

- sweet cream..............207

— testing by Babcock method..... 99

- texture of............222, 230

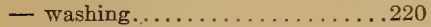

- white specks in .............208

- workers..................223

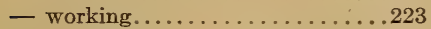

Buttermilk..................315

- characteristic appearance of . ..217

- Federal standard for.......... 366

- separation of from butter...... 219

Butyric acid...............20, 23

- fermentations............113, 118

Butyrin.................. 20

Butyrometer................ 93

By-products of dairy ............ 315

Caked udder............... 38

Calcium chloride..............248

- oxalate....................248

Calibration of Babcock glassware....359

- of glassware in Babcock test... . 99

Camembert cheese............295

Canadian cattle, French.......... 75

- Club cheese...............279

Capacity of cows............ 41

- relation of external form to..... 43

Capric acid................ 23

Caprilin..................... 20

Caprin..................... 20

Caproic acid............... 23

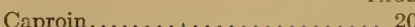

Caprylic acid................... 23

Caramel ice cream............. . 310

Care of cows................ 155

- - milk utensils.............. 160

- - stable................. 156

Careno, quoted............. 26

Casein.................23, 233

- coagulation of ..............234

— dried..................... . 320

- formation of............. 7

Cattle, Alderney............. 66

- Ayrshire............... 69

- Brown Swiss............... 73

- Devon..................... 74

- Dutch................. 67

- Dutch Belted ............ 74

— feeding dairy ........... 47

- French Canadian. ........... 75

— Guernsey...............6. 65

- Holstein................. 66

- - Friesian.............6.66 66

- Jersey................. 64

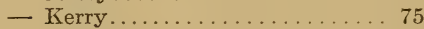

— Lakenvelder.............. 74

- Normandy................ . 75

— Red Polled............... 73

— Shorthorn ................ 71

- Simmenthal............. 75

Caustic potash............... 102

— soda.................. 102

Cells, secreting............. 3

Cement floors................. 330

Centigrade thermometer ......... . 349

Centrifugal, Babcock........... 94

- butter-worker.............225

- creaming................ 177

- force, relation to complete creaming............... 181

- separation................ 177

- - theory of............... 179

— separator, efficiency of . . . . . . 190

- - theory of ............. 180

- system.................. 164

Certified milk............... 149

- - standards............ 151

Cheddar cheese..............249

— English...............280

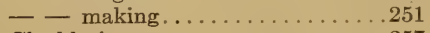

Cheddaring.................. 257

— process...................249

Cheese......................233

- Act, Filled............ 345, 382 
Cheese aëration of inilk $\ldots \ldots \ldots \ldots 242$

- American home-trade .........270

- and butter factories combined... 335

- Babcock test for . . . . . . . . . . 359

— bandage..................263

- Brie .....................295

- Camembert................295

- Canadian Club.............279

- Cheddar................249

- Cheshire............... 280, 286

- Club House.................279

- color of................268

- composition..............267

- cottage....................322

— cream...................270

- curing..................264

- curing in cold storage.........265

- curing-rooms, construction of . . .334

- Derbyshire............280, 288

— D'Isigny................297

— dividends on the fat basis.....239

— double Gloucester... . . . . . . . .280

— dressing.................. 263

- Dutch...................... 322

— Edam.................290

— Emmenthaler..............289

- English............... . 280

- English Cheddar.............280

- estimation of fat in by Babcock test................... 99

- factories.................. 326

- factories, construction of...... 332

— factory system.............. 341

— fancy..................270

- Federal standard for...........367

— filled................... 345

— filled law ................... 382

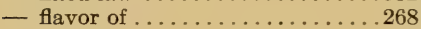

— "flinty break"............. 268

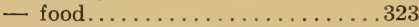

— formation of rind...........263

- Gloucester, single and double. .280

- Gouda.....................294

- Gorgonzola................. . 289

- Gruyere..................289

- hard.....................269

- Imitation Swiss................ 279

- Lancashire............. 280, 288

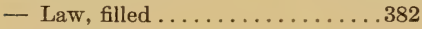

- leaky...................271

- Leicestershire.............280, 288

— Limburger...............277

- making................233
PAGE

Cheese making, Cheddar........251

— - cheddaring or matting.....2.257

— Cheddar process............249

- - cooking the curd.........255

— - cooling milk...........241

— - curing..................263

— - cutting curd.............253

— - "gassy" curds.............266

— - grinding curd...........259

- $\longrightarrow$ heating................255

- - loss of fat in...........240

- — overripe milk..........266

- - pressing ..............261

— - quality of milk for . . . . . . 234

— - removal of whey.........249

— - rich and poor milk in......238

— - ripening milk for.........242

— - salting the curd.........260

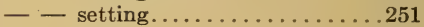

— - starters..............246

— - temperature of setting. . . . 251

— - undesirable fermentations in .265

- Meadow Sweet..............279

- Neufchatel.................273

- Parmesan................ 298

- Philadelphia Cream..........277

- picnic..................273

- pineapple.................273

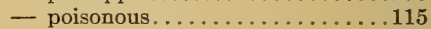

- Pont L'Eveque............. 297

- Port du Salut..............298

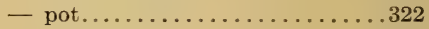

- prepared................279

- press......................262

- pressing....................261

- quality of ................ 267

- ratio of fat to casein..........235

- relation of fat in milk to .......236

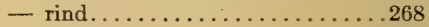

- ripening..................264

- Roquefort................ 294

- sage.................272

- Schweitzer ...............289

- score card for............. 268

— single Gloucester ........... 280

— skimmed..............269, 345

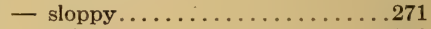

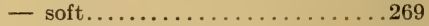

— solids concerned in making. . . . . 233

- square cream............. 277

- state brands for............... 345

- Stilton..................280

- stirred curd................270 


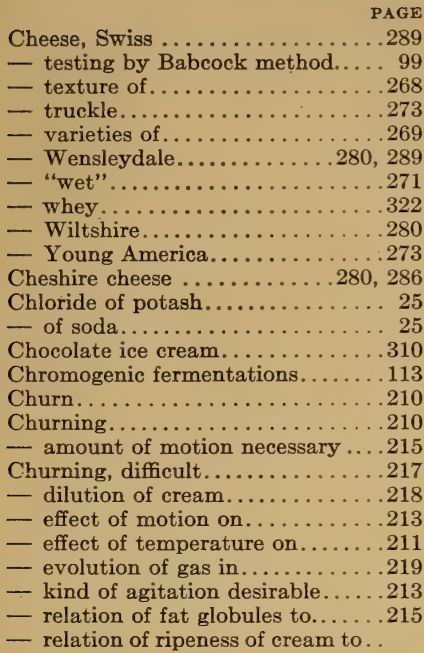

207,211

- - - temperature.........211

- - - viscosity to.............210

- rise of temperature in........219

Churn, oil test............... 84

Churn tests................ 83

Cistern, milk.............. 5

Citric acid................ 26

Classification of ice cream.........300

Cleaning Babcock test glassware. . . 107

— dairy tinware ............. 136

- glassware ................. 130

- utensils.................... 13

Cleanliness....................... 135

Clotted cream, Federal standard for . 366

Cloth strainers................137

Club-house cheese.............279

Coagulation of albumin.........24

- - casein............24, 234

- - fibrin................. 24

Coccus.................... 109

Cochran, C. B., mentioned........ 91

- method................. 91

Coffee ice cream............... 310

- parfait.................311

Cold storage, curing cheese in.....265

Color fermentations. . . . . . . . . . 113
PAGE

Color of butter. . . . . . . . . . . 231

- - cheese................268

Colostrum................. 9, 18

— corpuscles................. 18

— test for.................. 18

Columbia separator............ 191

Combined butter and cheese factories.................... 335

Commercial ferments........... 199

- milk................. 36

Comparison of lactometer scales .. . 350

- - thermometer scales........ . 349

Composite sampling. . . . . . . . . . 102

Composition of butter . . . . . . . . .229

— - cheese...............267

- - colostrum............. 18

- - cream................... 148

- - milk................. 16

Concentrated milk............ 319

Concentrates............... 49

Concussion in churning . . . . . . 213

Condensed milk............... 316

- Federal standard for........ 365

— skim milk, Federal standard for.366

Conn culture...............200

- H. W., quoted................ 199

Connective tissue............. 3

Constituents of milk.......... 17

Construction of creameries....... 330

Continuous freezers............. 307

— pressure cheese press..........262

Control apparatus, Fjord's ...... 88

— of fermentations.............118

- - milk supply............. 145

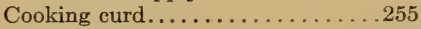

Coolers, milk................ 139

Cooley system................ 169

Cooling milk............138, 158, 241

Coöperative cow testing ........ 47

Cornell butter moisture test. . . . . 359

Cornevin, quoted............. 17

Corpuscles, colostrum.......... 18

Correction for lactometer temperature.................... 350

Corrosive sublimate............ 102

Cottage cheese................ 322

Counting bacteria.............133

Covered milk pails............. 158

Cow as commercial milk producer... 36

- effect of age on composition of milk................. 31

-1 - breed on composition of milk................... 33 
Cows, advanced registry........ 45

- care of ..................155

- contraction of milking habits... 41

- drying up................ 38

- feeding............... 47

- - standards for.......... 48

— grade................ 53

- holding up milk........... 5

- ideal ration for.............. 47

- individual capacity of........41

- milk................... 16

- number in United States. . . . . . 339

— official inspection of . . . . . . . . 143

- pure bred.............. 53

- relation of form to capacity..... 43

- requirements for registration.... 54

— score card for.............. 44

_ "scrub"................. 53

- selection of...............154

- testing associations..........47

- wedge-shaped form.......... 42

Cranberry mousse .............. 311

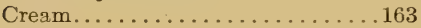

— cheeses................270

- - Philadelphia............277

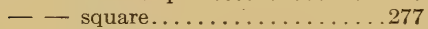

- composition of............... 163

- consistency of............. 147

- definition of .............. 163

- degree of ripeness necessary....204

- dilution in churning. . . . . . . . 218

- effects of ripening...........206

- estimation of fat by Babcock test ............... 97

- Federal standard for.......... . 366

- for consumption............... 146

- frothing or swelling..........218

- gathering system......... 83, 342

- gauges................. 78

- glasses................... 78

- homogenized..................... 303

- hydrochloric acid in ripening . . . 197

— ice.......................299

— ices.................... 300

- influence of oxygen in ripening, .197

— over-ripening ..............208

— pasteurized.................147

- pasteurizing.............202

- quality of for consumption. . . . 148

_ _ _ - ice cream...........303

- relation of ripeness to churning.................... 211

- - - temperature to ripening. . 203
PAGE

Cream ripening of . . . . . . . . 195

- - lactic acid in............. 196

- - temperature of..........203

- screw................... 185

- separation of ................ 163

- standardization of ......... 353

— standards for.............. 148

— state standards for......... 368

— "swelling" of . . . . . . . . . . 218

— testing by Babcock method .... 97

- test bottles.............. 96

- use of starter in ripening...... 198

— visco.................. 148

Creameries.................. 326

- arrangements of ............ 327

- construction of ............. . 330

- gathered cream............ 342

— gravity system............ 327

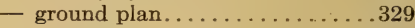

- location of ............... 326

— pumping system................328

- water supply of..............331

Creamery butter, imitation. . .346, 347

— systems.................. 341

Creaming by dilution...........173

— centrifugal....................

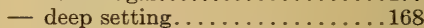

- effect of delayed setting........171

- — - dilution on............ 173

- gravity..............164, 165

- losses in deep setting.......... 169

- - - shallow pan..............168

- necessity for................ 165

- relation of centrifugal force to complete................ 181

- — - centrifugal to...........182

- — - fat globules to.........166

- - - inflow to complete......184

- - - solids not fat to.......166

_ - - viscosity of milk to...... 167

- shallow pan ................ 167

- systems of................ 164

- theory of centrifugal..........18c

- theory of deep setting system .. 168

Creamometers................. 78

Culture, Conn...............200

- starters..................201

Curd, cooking...............255

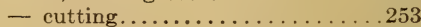

- fast working................ . 266

- floating .................. 265

_ "gassy"................... . 265

- grinding ................259 
PAGE

Curd heating................. 255

- in Babcock test............. 105

- knives...................254

- matting or cheddaring. ... . . . 257

- membrane............... . 255

- mills.................... . 259

- salting.................260

$-\operatorname{sink} \ldots \ldots \ldots \ldots \ldots \ldots . \ldots . . \ldots 257$

- test, Wisconsin........... . 357

Curds, washing or soaking. ....... 272

Curdling, sweet.............113

Curing cheese................264

- cheese in cold storage. . . . . . . . 265

- rooms, construction of.........334

- - subearth duct.......... . 244

Cutting curds............... 253

Dairy breeds................6 63

— buildings, farm............ . 326

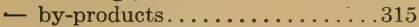

— cattle, feeding............. . 47

— factory system............. . 341

- herd, formation of.......... 53

- — "grading up"............ 58

- - maintenance of.......... 54

- law, New York............... 387

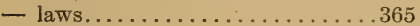

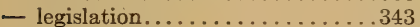

- markets................. . 346

— products, legal standards for....365

- references..................4404

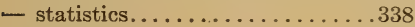

- type................... . 42

Dairying, associated. . . . . . . . . . 340

Danish separator, large and small . . 191

- Weston separator.............191

Dean, H. H., quoted. . . . . . . 142, 237

Decinormal alkali......... 204, 351

Deep setting creaming........... 168

- - system............ 164, 168

- - - theory of............ 169

D'Isigny cheese... . . . . . . . . . . 297

De Laval Baby separator. . . . . . . . . 193

- Gustav, mentioned.......... 179

- lactocrite................ . . 87

- separator...........185, 187, 191

- separator, Alpha No. 1 . . . . . 192

— standard separator........... 193

Delayed setting, effect on creaming ................. 171

Derbyshire cheese... . . . . . 280, 288

Detachable neck test bottle....... 96 Determination of bacteria in milk. . 125
PAGE

Determination of lactic acid . .205, 351 Detection of taints and fermenta-

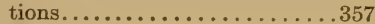

Devon cattle ............... 74

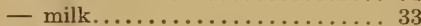

Dextrose.................... 25

Diameter of fat globules......... 23

Difficult churning............217

Dilution creaming............ 173

- separator................. 177

Dioxystearic acid............. 23

Dipping milk............ . . . . 139

Dises alpha .............. 187

Disease germs.............113, 122

- relation of pasteurization to . . . 142

Diseased herds-official inspection a prevention............... 142

Dishes, petri................ 134

Disinfectants, use of ........... 156

Double Gloucester cheese.........280

- neck test bottle............. 96

Drainage, air ............... . 327

"Dressing" cheese................263

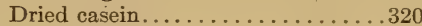

"Drying off" cows............... 38

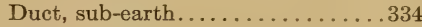

Ducts, milk...............2, 15

Dust in stable, removal of . . . . . . . 156

- relation of to bacteria......... 119

Dutch Belted cattle............ 74

— cattle................. 67

- cheese.................... . 322

Edam cheese.................290

Effect of delayed setting on creaming. . . . . . . . . . . . 171

- _ dilution on creaming. . . . . . 173

Efficiency of centrifugal separator. . 190

Electricity as a germicide......... 121

Electrolysis of milk............ 121

Emmenthaler cheese... . . . . . . . 289

Emulsion.................23, 163

English Cheddar cheese.........280

- cheeses....................280

Escutcheon................ 56

Essential oils in milk. ............ 20

Estimation of solids not fat... .107, 350

— of total solids..........107, 350

Ether-like flavors. . . . . . . . . . . . 197

Eureka butter worker. . . . . . . . . . 223

Evaporated cream, Federal standard..........................

- milk..................... 318 
Evaporated milk, Federal standard for .................... 365

Ewe's milk.............. 16 Ewe's milk cheese, Federal standard for ................... 367

- - Federal standard for .........366 Excrement, animal, relation of bacteria to ................ 119

Experiment Station Bulletins. . . . . 404

Extract, rennet.............248

Factories, arrangement of cheese. . .332

— butter and cheese........... . . 326

- butter, arrangement of . . . . . . . 327

- - construction of ...........330

- cheese, construction of........332

- gathered cream............ 342

Factory butter................ . 346

- system, development of.......341

Fahrenheit thermometer......... . 349

Failyer, Professor, mentioned.... . 89

— and Willard, mentioned ....... 89

- — - method............. 89

Fancy cheese...............270

Farm dairy buildings. .......... 335

Farrington's alkaline tablets...... .

$124,305,351$

- E. H., mentioned.............204

- - - quoted............123, 199

Farrow cows, milk from......... 28

Fast-working curds...........267

Fat basis in cheese dividends. . . . .239

- estimation in cheese by Babcock test.

— formation of ............ 8

- globules............... 8

- globules, diameter of ......... 23

- - relation of to churning.....215

- in milk, relation to cheese made. 236

- loss of in cheese-making. ......240

- milk or butter, Federal standard.366

- temperature of reading in Babcock test............95, 106

Fats.................. 20

- effect of feed on............ 30

- - period of lactation on.... 27

- formation of ............ 8

— gravimetric determination of . . 77

— non-volatile.............. 22

- volatile.................. 197

Fatty tissue.............. 3

Febrile condition, effect on milk... 29

Federal standards..............365
PAGE

Feeding dairy cattle......... 47

- standards for cows........... 48

Ferment, preparation of artificial...354

Fermentation; abnormal..........113

Fermentations, albuminoid........117

— alcoholic................. 25

- butyric...............113, 118

- chromogenic..............113

- control of ................ 118

- detection of......................

- gaseous..................... 117

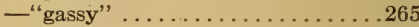

- germs of . . . . . . . . . . . . . . 109

- lactic.............. 113, 115, 266

- of milk................. 108

— normal..................113

- peptogenic............113, 117

- poisonous.................115

- putrefactive...........113, 117

Ferments, undersirable in cheese making...............265

- commercial.................... 199

- Hansen's lactic................... 199

- preparation of ripening........ 354

— soluble.................234

Feser's lactoscope ............ 86

Fibrin.................23, 171

Filled cheese................... 345

- - law.................345, 382

Fillers for ice cream.......... 305

Finish of butter..............232

First milk................ 30

Fission.................... 109

Fjord's control apparatus........ 88

- Professor, mentioned.......... 88

Flavors due to food............ 21

— of butter..................230

- - - relation of wash water to. 223

— - cheese ${ }_{1}$...............268

- bad in milk .............. 143

— ether-like................ 197

- for ice cream................. 304

— producing germs............ 197

Fleischmann, quoted ......... 17

Fleshy udder............... 3

"Flinty break" of cheese........268

Floating curds..............265

Floors, cement.............. 330

Follicles, ultimate . . . . . . . . 3, 6

Food as a factor in milk production 47

- avoiding flavors due to....... 21

- effect of on flavor of milk......21

- - on composition of milk..... 30 
PAGE

Food effect on quality of fat...... 30

Foods, necessity for succulent..... 50

— palatability of ........... 50

- relation of proteids to non-proteids................ 51

Forage-relation of dry to bacteria. 119

Fore milk and strippings........ 30

Formalin .................. . 121

Formation of cheese rind........263

Formulæ for total solids . . . . . . . 350

Frappes................ . 300, 301

Fraser cheese press... . . . . . . . . 262

Freezers. . . . . . . . . . . . . . . . . 306

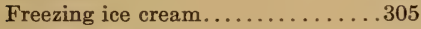

- mixtures. . . . . . . . . . . . . 306

French Canadian cattle.........75

Fright, effect on milk secretion......14

Frothing of cream............218

Fruit ice cream ................. 301

— — - Federal standard for... . .367

Galactose................. 25

Gang cheese press.............. 262

Gaseous fermenta ions. . . . . . . . 117

Gases in milk. . . . . . . . . . . . . . 138

Gas, evolution of in churning. . . . . . 219

"Gassy" curds... . . . . . . . . . . . 265

Gassy ferments in Neufchatel cheese.................274

Gathered cream system. . . . . . . . . 341

Gauges, cream.............. 78

Gelatine.......127, 128, 129, 131, 305

- lactose... . . . . . . . . . . . . 129

Gerber, mentioned ............. 92

Gerber's method... . . . . . . . . . . . 92

Germs, destruction of in milk.... 120

- diseases................ 113

- flavor producing............ 197

- lactic acid............... . 115

- of disease............... . . 122

- of fermentation............. 109

Glands, mammary............ 1

Glasses, cream.............. . 78

Glassware, Babcock, calibration of.. 99

- - test................. 95

- - - accuracy of.......... 99

- calibration of Babcock.........359

- cleaning.................. 130

- - Babcock............... 107

Globules, fat................ 23

Gloucester cheese.............. . . 280

Glycerides................... 22

Goat's milk. ..............16, 36
Goat's milk, Federal standard for . .366

- cheese, Federal standard for. 367

Gorgonzola cheese. . . . . . . . . . . . 289

Gouda cheese. . . . . . . . . . . . . . . . 294

Grades... . . . . . . . . . . . . . . . . . 53

"Grading up" the herd........... 58

Grain of butter............ . . 230

Granite ice cream. . . . . . . . . . . . . . . . . . . . . . . . . . .

Granules, size of butter. . . . . . . . . 221

Gravimetric analysis... . . . . . . 77

Gravity, creaming..........164, 165

- separators................ 177

— specific............79, 82, 349

- system of creamery construction. 327

Grinding curd. . . . . . . . . . . . . 259

Gruyere cheese ... . . . . . . . . . . . 289

Guernsey cattle.............. 65

- milk................. 33

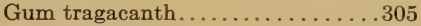

Gurler, H. B., quoted.......... 200

Half blood, definition of......... 53

Hansen's lactic ferment........... 199

Hard cheeses. . . . . . . . . . . . . 269

Harris curd mill ... . . . . . . . . . . . 259

Hay, relation of bacteria to........119

Heating curds............... 255

Heat, sexual, effect on milk secretion 14

Heeren's pioscope. ............ 85

Hegelund, mentioned. . . . . . . . . 40

— method of milking.......... 40

Herd, formation of dairy......... 53

— "grading up" the............ 58 58

- maintenance of dairy........ 54

— official inspection of diseased . . 143

Hill, Janet McKenzie, quoted..... 300

Holding up milk............ 5

Holstein cattle.............. 66

— Friesian cattle............ 66

- - milk. . . . . . . . . . . . . . 33

Home trade cheese. . . . . . . . . 270

Homogenized milk and cream.... . . 303

Horizontal separators. . . . . . . . . . 179

Hot iron test. . . . . . . . . . . . . 358

Hueppe, mentioned............ 115

Hydrochloric acid in ripening cream 197

Hydrometer. . . . . . . . . . . . . . . 79

Ice cream. . . . . . . . . . . . . . . . 299

- - caramel............... . 310

- - chocolate...............310

- - classification.............300

- - coffee..................... 310 
PAGE

Ice cream, federal standard for . . . 367

— fillers for. . . . . . . . . . . . 305

- - flavors.................. 304

- - freezers. . . . . . . . . . . . . 306

- - freezing and packing .......305

- - gum tragacanth for........ 305

- - lemon.................310

- macaroon............... . 310

— - maple................ . 310

- mint. ................ . 310

— - orange................ . 310

— — quality of cream for. . . . . . . 304

- - recipes................. . 308

— - score card for............312

- - strawberry............. . 310

- - tutti frutti.............. . 311

- - walnut............... . 310

Ices . . . . . . . . . . . . . . . . . . . . . . . . . . . . . . . . . . . . . . . . . .

Ideal ration for cows. . . . . . . . . 47

Imitation creamery butter . . . . . . 346

- Swiss cheese. . . . . . . . . . . . . .279

Inbreeding ................ 61

Indicator, litmus. . . . . . . . . . . . . . 351

— phenolphthalein ......... 204, 351

Individual capacity of cows.......4 41

Infection, prevention of . . . . . . 118

Inflow, relation of to complete creaming................ 182

Iowa Station test.............. 90

Iron clad tinware ............. 135

Iron, phosphate of ........... 25

Jersey cattle.................. 64

- milk.................... 33

Jordan, W. H., quoted...........173

Jumbo separator. . . . . . . . . . . 191, 192

Keeping quality, relation of lactic acid to................. 207

Kephir. . . . . . . . . . . . . . . . 324

Kerry cattle................ 75

Knives, curd................254

Koenig, quoted............... 17

Koumiss................. 16, 324

—. Federal standard for. .......... 367

Laboratory, bacteriological........126

Lactation period, effect on composition of milk.......... 27

Lactic acid.....25, 116, 196, 208, 241

- - determination of ......205, 351

- - formation of ............. 198

- - germs................ 115
Lactic acid relation to keeping

quality of butter..........207

— fermentations. . . . . . .113, 115, 266

- ferments, Hansen's. . . . . . . . . 199

Lactobutyrometer, Marchand's.... 85

Lactochrome.................. 26

Lactocrite, De Laval. . . . . . . . . . 87

Lactometer................ 79

- Board of Health..........80, 349

- comparison of scales.......... 80

- correction for temperature..... 81

- Quevenne..............80, 350

— scales, comparison of ......... 80

- temperature correction for . . . . 350

Lactoprotein.................. 26

Lactos...................... . 301

Lactoscope................ 86

Lactose.................. 25

- agar.................... . . . . . . . . . . . . . . . . . . . . . . . .

- gelatine.................. 129

Lakenvelder cattle... . . . . . . . . . 74

Lancashire cheese... . . . . . . 280, 288

Large Danish separator.... . . . . . . 191

Lauric acid . . . . . . . . . . . . . . . . . 23

Laurin................... . 20

Law, dairy. . . . . . . . . . . . . . 365

— filled cheese. ........ . . . . . 382

— New York dairy... . . . . . . . . . 387

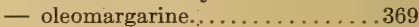

"Leaky cheese"... . . . . . . . . . . .271

LeFeldt and Lentsch, mentioned... 178

LeFeldt and Lentsch separator ..... 178

Leffman and Beam, mentioned..... 92

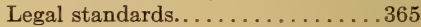

— - for milk............... 145

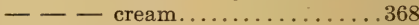

Legislation, dairy . . . . . . . . . . . . 343

Leicestershire cheese. . . . . . . . 280, 288

Lemon ice cream.............. 310

- sherbet................... 311

Lentsch and LeFeldt, mentioned... 178

Light in stables, minimum requirements................... 153

Limberger cheese.............. 277

Lime, phosphate of........... 25

Lime salts......... . . . . . . . . 247

Litmus. . . . . . . . . . . . . . . . . . . 129

- test..................... . . . . . . . . . . . . . . . . . .

Loss of fat in cheese making. . . . . 240

Lymphatics................. 3

Macaroon ice cream............ 310

MacPherson curd mill.........260 
Mammæ................... 1

Mammalia.................. 1

Mammary glands.............. 1

Mann's acid test ...............204

- A. G., mentioned......... . . . 204

Maple ice cream............ . 310

Marchand's lactobutyrometer .... 85

Mare's milk. . . . . . . . . . . . . . 16

Marketing butter.............. . 227

Market milk. . . . . . . . . . . . . . 135

Markets, dairy... . . . . . . . . . . 346

Marschall rennet test.... . . . . 244, 358

Mason butter worker. . . . . . . . . . 224

Maternity effect on milk secretion

Matting.................. . . 257

Meadow sweet cheese . . . . . . . . . 279

Media, bacteriological. . . . . . . . . 127

Media, standardization.......... 128

Medium Brown separator. . . . . . . 191

Membrane on curd........... . 255

Method, Cochran's............. . 91

— Gerber's.............. . . . . 92

- of Failyer and Willard. . . . . . . . . 89

- - Leffman and Beam... . . . . . 92

- Parson's.............. . . . . . 90

- Short's........... . . . . . . . 89

- Soxhlet's................ . . 87

Metric system... . . . . . . . . . 363

Milk, aëration of . . . . . . . . . . . 138

— aërators. . . . . . . . . . . . . . . 139

- albuminoids of ............ 23

- analyses................. 17

- as affected by maternity...... 35

- ash in................. 25

- asses ${ }^{\prime} \ldots \ldots \ldots \ldots \ldots \ldots \ldots \ldots$. . . . 36

- Ayrshire.......... . . . . . . . 33

- bacteria in............... 111

- bad flavors in.............. . 143

- bitter................ 113, 143

- bottles............. . . . . . 142

- bottling.............. 142, 160

- certified.................... 149

- cistern................ . 5

- commercial.............. 36

- composition affected by age of cow.................... 31

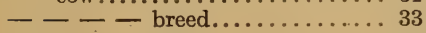

$--\div-$ food ................. 30

Milk concentrated ............. 319

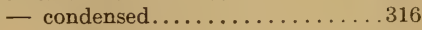

- constituents of ........... 17

- control of animal over secretion. 14

- coolers.................... 139

- cooling...........138, 158, 241

- cows.................. 16

- definition of .............. 1

- destruction of germs in........ 120

- determination of bacteria in. . . 125

- Devon... . . . . . . . . . . . . . 33

- difference in first and last drawn. 30

- dipping................. 139

- diurnal variation........... . 29

- ducts................... 5

- duration of flow........... 11

- effect of age on composition.....31

— - breed on composition... 32

- — food on flavor of...... 21

- - pregnancy on flow...... 39

- evaporated.....................................

- ewes.................... 16

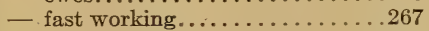

- fat, Federal standard for. . . . . 366

- fats................... 20

- Federal standard for.......... 365

- fermentations............... 108

- first and last drawn ........ . 30

- for cheese making... . . . . . . 233

- for pasteurization. . . . . . . . . 352

- from farrow cows.......... 28

- - males............... 10

- organs other than the mammary glands............. 10

— — spayed cows............ 28

— virgin animals........... 10

- gases in............... . . 138

- goat's................. 16, 36

- Guernsey................ . . 33

- holding up.............. 5

- Holstein-Friesian. . . . . . . . . . 33

- homogenized... . . . . . . . . . . 303

- influence of food on composition 30

- Jersey................... . 33

- legal standards for. . . . . . . . . 145

- mare's.................. 16

- market................. . 135

- morning and night.......... 28

- overripe................ 266

- pails, covered.............. 158

- paying for according to percent-

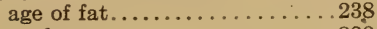


Milk preservaline.............102

- production of ............... 35

- - relation of breed to........ 52

--- food to........... 47

- quality of................ 144

- record, largest............. 37

- relation of viscosity to churn-

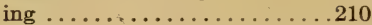

- reservoir................ 5

- rich and poor in cheese making..238

- ripening.................. . 242

- ropy or slimy.............. . 113

- sampler, Scovell aliquot.......103

- secretion of .............. 7

- - effect of animal on........ 14

-- - fright on.......... 14

- - - maternity on...... 37

- - - pregnancy on....... 12

- - - sexual excitement on . 14

- - incentives to............ 9

- relation of blood to........ 11

- - of nervous organization to.................. 14

- - - of parturition to....... 9

- selection of for pasteurization. . 121

- sherbets............... . . . 301

- - pineapple................ 311

- Short Horn............... 33

- $\operatorname{signs} . . \ldots \ldots \ldots \ldots \ldots \ldots \ldots \ldots$

- skimmed................. . 315

- $\operatorname{slimy} \ldots \ldots \ldots \ldots \ldots \ldots . \ldots \ldots 113$

- solids................. 16

- concerned in cheese making. . 233

- souring of................116

- specific gravity of ........... 19

- standardization of.......... . 353

- standards for certified........ 151

- State standards for... . . . . . . 368

- sterile................ . . . 109

- straining............ . . . 137

- sugar............... 25, 321

- - formation of .......... 9

- supply, control of...........145

- testing................ 77

- tests, ${ }^{-B a b c o c k} . . . . . . . . . . .991$

— - Beimling.............. 92

- - Butyrometer............ 93

- - Cochran's............... 91

- - Failyer \& Willard's.... . . . 89

- - Gerber's................ 92

- - history of ............ 78

- - Iowa Station............. 90

- - Parsons'................ 90
PAGE

Milk tests, Short's ............ . 89

- thief..................... 101

- utensils, care of ........... 160

- variation in .............. 17

- - due to febrile condition.... 29

- - in quality............. 27

- veins................... 56

- viscosity of ................ 210

Milking.................... 39, 157

- frequency of ............. 13

- habits of cows............ 41

- importance of complete. . . . . . 12

- — - regularity........... 13

— limit of frequency.......... 40

— rapidity of ............... 14

Mill, curd. ................. 259

Minor dairy breeds............ 63

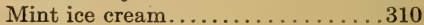

Moisture test, Cornell butter. . . . . . 359

Molds... . . . . . . . . . . . . . . . . 109

Monrad, J. H., quoted. . . . . . . 277, 323

Monrad rennet test........ . 243, 358

Moore, quoted...............200

Morning's milk. . . . . . . . . . . . . . . 28

Mortensen, quoted. . . . . . . . . . 301

"Mother" starters. . . . . . . . . . . . . . 202

Mottled butter................ 226

Mousse, cranberry.............. . 311

Mousses................ 300, 301

Myristic acid............... 22

Myristin.................... 22

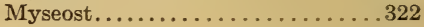

$\frac{\mathrm{n}}{10}$ alkali $\ldots \ldots \ldots \ldots \ldots \ldots \ldots \ldots \ldots \ldots$

Neapolitan ice cream.......... 300

Nervous organization, relation to milk secretion............ 15

Nesselrode pudding. . . . . . . . . . 311

Neufchatel cheese... . . . . . . . . 273

Neumann, quoted.............. 102

New York dairy Law ... . . . . . . . 387

Night's milk. . . . . . . . . . . . . . 28

Nipple... . . . . . . . . . . . . . 2

Nitrate of soda............... 102

Non-volatile fats........... 20, 22

Normal sodium hydroxid. ... . . . . 130

- fermentations .............. 113

Normandy cattle............ 73

Nut ice cream. . . . . . . . . . . . . 301

Nut ice cream, Federal standard. . .367

Nutrient bouillon............. 127

Nutritive ratio.............. 51 
PAGE

Odor, animal................ 138

"Off flavor" starters... . . . . . . . . 201

Official inspection of cows. . . . . . . 143

Ohlssen's test bottle........... 96

Oil test churn................ 84

Oils, essential in milk.......... 20

Oleic acid................... 22

Olein. . . . . . . . . . . . . . . . . 22

Oleomargarine... . . . . . . . . . . 344

Oleomargarine law....... . . . . . . 369

Oliver, quoted............... 17

Operation of milking............ 39

Optimum temperature... . . . . . . 110

Orange ice cream............ . 310

Overripe milk. ........... . . . 266

Overripened cream............208

Oxygen in ripening cream. . . . . . 197

Packages, butter... . . . . . . . . . 228

Packing butter. . . . . . . . . . . . . . 227

- ice cream................ 305

Pails, covered milk. . . . . . . . . . 158

Palatability of foods........... 50

Palmitic acid............... 22

Palmitin.................... 22

Paper coil method, Adams........ 78

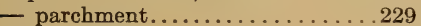

Parfait, coffee..................311

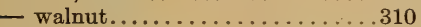

Parfaits.................. . 300, 301

Parmesan cheese...............298

Parsons, C. L., mentioned. . . . . . . . 90

- method................ 90

Parturition, relation of to milk secretion.............. 9

Pasteurization................ 143

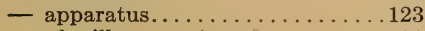

- of milk................... 121

- selection of milk for . . . . . 123, 352

Pasteurized cream......... 147, 202

- milk, Federal standard for. . . . 365

Patrick, Geo. E., mentioned.... . . . 90

Paying for milk according to percentage of fat............239

Pearson, R. A., quoted........... 353

Peptogenic fermentations.... . 113, 117

Period of lactation............. 37

Petri dishes....... . . . . . . . . . . 134

Phenolpthalein....... . . . . . . . 204

- test.................... . 351

Philadelphia cream cheese... . . . 277

- ice cream.................. . 300

Phosphate of iron........... . 25
PAGE

Phosphate of lime............. 25

— of magnesia............... 25

- of potash................ 25

Picnic cheese............... . 273

Pietertje 2 nd, record of. ........ 37

Pineapple cheese...............273

— milk sherbet................ . 311

- souffle................... . 311

Pioscope, Heeren's . . . . . . . . . . . 85

Plans for cheese factories.........327

— - creameries...............327

Plating..................... . 132

Pohl curd mill. . . . . . . . . . . . . 260

Points, scale of for cows......... . 44

Poisonous fermentations..........115

Polled cattle, red.............. 73

Pont L'Eveque cheese . . . . . . . . . . 297

Port du Salut cheese............. 298

Potash, caustic................. 102

- chloride of ............... 25

- phosphate of............... 25

Potassium bichromate... . . . . . 102

- chloride................ 25

Pot cheese. . . . . . . . . . . . . . . 322

Precipitation of casein.......... 24

Pregnancy, effect on composition of milk............... 27

- - on milk secretion....... 12, 39

Preparation of artificial starter. . . . 354

Prepared cheese.............. 279

Prepotency.................. . 59

Preservaline, milk. . . . . . . . . . . 102

Preservatives in composite sampling 102

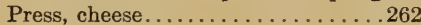

Pressed tinware................ 136

Pressing cheese.............. . 261

Prevention of infection..........118

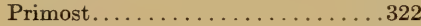

Print butter. . . . . . . . . . . . . . .229

Process butter. . . . . . . . . . . . . 346

— - Federal standard for. ....... 366

Production of cows, value of records. 45

— of milk, relation of food to..... 47

Proteids, relation of to non-proteids in food.............. 51

Ptomaines.............................

Pudding ice cream............... . 301

Pumping system of creamery construction.................. 328

Punches................ 300, 301

Pure breds.................. 53

- - registration of . . . . . . . . 54

Putrefactive fermentations... . 113, 117 
Quality of butter.............229

— of cheese................267

— of cream for consumption......148

— of cream for ice cream. . . . . . .303

— of milk for cheese making. . . . . 234

- of milk for consumption........144

— - - legal standards for...145, 367

- - - variations in.......... 27

Quevenne lactometer........ .80, 350

- mentioned................ 80

Rancid butter.............. 20

Ratio, nutritive............ 51

Ration, "balanced"............. 51

- for cows, the ideal............ 47

Reaction, amphoteric........... 108

Recipes for ice cream.......... 308

Records of production, value of.... 45

Red Polled cattle............... 73

References..................404

Register of merit........... 45

Registration of pure bred animals.. 54

Rennet.........234, 247, 252, 305

- extract..................248

- relation of temperature to activity of ...............247

- tests.................243

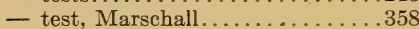

- - Monrad................. 358

Renovated butter............ 346

Renovated butter, Federal standard for.................. 366

Reports and bulletins of Agricultural Experiment Stations..... 404

Reproduction by fission..........109

Richmond formula for total solids. 350

Richmond, quoted............. 18

Rind of cheese...............268

- - - formation of..........263

Ripeness, churning cream of different degrees..............207

Ripeness of cream, relation to churning........................

Ripening cheese..............264

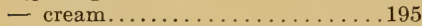

- - amount necessary.........204

- - effects of...............206

- - hydrochloric acid in....... 197

- - influence of lactic acid.....196

- - relation of oxygen to........197

- - temperature of.............203

- - use of starters in..........198

- ferments preparation of.......354
PAGE

Ripening milk, for cheese making. . 242

Ropy milk................. 113

Roquefort cheese............. . 294

Roughage................. 49

Rules and tests............... 349

Rudimentary teats............ 56

Russell, H. L., quoted. . . 123, 147, 200

Russian separator Sharples........193

Rusty tinware..............136

Sage cheese................272

Salicylic acid............... 121

Salt..................226, 306

Salting butter...............224

- curd.....................260

Salts of lime...............247

Sampler, Scovell aliquot milk......103

Sampling, composite............ 102

Sanitary stables............... 152

Scale of points for butter........232

— - - - cheese.............268

- - - - cows.......... 44

Scales, comparison of lactometer. 80

__ - of thermometer........... . 349

Schmierkase.................. 322

Schweitzer cheese.............289

Score card for butter.............232

- $-\ldots$ cheese..............268

- - - ice cream...............312

Scovell aliquot milk sampler.......103

Scovell, M. A., mentioned.........103

"Scrub" cows................. 53

Secretion of milk............ 7

Selecting milk for pasteurizing. .... 123

Selection of breed............ 52

— of bull.................. 55

— of cows.................. 154

Separation, centrifugal..........177

— of cream..................163

Separator, Accumulator........... 191

— adjustable.................. 191

- Alexandra Jumbo........... 191, 192

- Alpha B................... 191

- Alpha Baby.................191

- Arnold's.................... 191

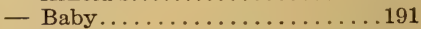

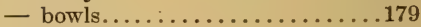

- Columbia.................. 191

- Danish Weston.................. 191

- De Laval...................... 191

- - Alpha No. 1............... 192

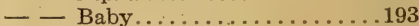

- - Standard.................... 193 
PAGE

Separator dilution ............. 177

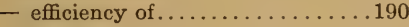

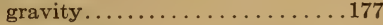

- horizontal. . . . . . . . . . . . . 179

- large Danish. . . . . . . . . . . . . . 191

- LeFeldt and Lentsch . . . . . . . . 178

- mechanical devices in bowl of . 185

- medium Brown. . . . . . . . . . 191

- Sharples... . . . . . . . . . . . . . 191

- - Russian.... . . . . . . . . . . 193

- Simplex................. . 186

- slime.................. . 181

- small Danish ... . . . . . . . . . . 191

- system.......... . . . . . . . . 164

- theory of centrifugal.......... 180

- tubular. . . . . . . . . . . . . . . 188

- United States. . . . . . . 187, 191, 193

- Victoria........ . . . . . . . . . 191

Setting, effect of delay on creaming. 171

- milk for cheese making.......251

Seven-eighths blood, definition.... . 54

Sewers... . . . . . . . . . . . . . . 331

Sexual excitement, effect on milk secretion............. 14

Shallow pan creaming......... 167

- - system. . . . . . . . . . . . . . 164

Sharples Russian separator. . . . . . 193

- separator................. . 191

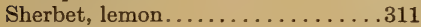

Sherbets... . . . . . . . . . . 300, 301

Short, F. G., mentioned..........89

Shorthorn cattle............. 71

Shorthorn milk.............. 33

Short's method............... 89

Simmenthal cattle............ 75

Simplex separator... . . . . . . . . 186

Single Gloucester cheese. . . . . . . . 280

Size of butter granules. . . . . . . . 221

- - fat globules............ 116

Sink, curd................. 257

Skim milk. ................ 315

- - cheese............. 269, 345

— - Federal standard for.....367

— - Federal standard for........ 365

— healthfulness of . . . . . . . . . 144

- - testing by Babcock method. 98

- - test bottle..............996

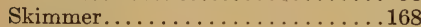

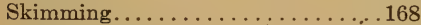

Slime, separator. . . . . . . . . . . . 181

Slimy milk. . . . . . . . . . . . . 113

"Sloppy" cheese. . . . . . . . . . . . . . .271

Small Danish separator. . . . . . . . . 191
Soaking curds............... . 272

Soda, caustic................ 102

- chloride of............... 25

Sodium hydroxid, normal ......... 130

- nitrate................... 102

Soft cheese... . . . . . . . . . . . . 269

Solids concerned in cheese making . . 233

- estimation of total......107, 350

— formulæ for total... . . . . . . 350

- not fat, formulæ for. . . . . . . . 350

- - - relation to creaming. . . 166

- total, determination of ....... 77

Soluble ferments. . . . . . . . . . . . . 234

Souffle, pineapple . . . . . . . . . . . 311

Souffles.................... . 301

Souring of milk..............116

Soxhlet's method............ 87

Spayed cows, milk from......... 28

Specific gravity..........79, 82, 349

一 - of milk.............. 19

Speed of bowl, relation of, to complete creaming............ 182

Sphincter muscle............4, 5

Spirillum.................... 109

Spore................... 110

Square cream cheese........... 277

Stable, care of . . . . . . . . . . 156

- minimum light requirernent.... 153

- removal of dust in . . . . . . . . 156

Stables, sanitary. . . . . . . . . . . 152

— standard air space.......... 153

Standardization of media........ 128

— of milk and cream........... 353

Standard separator, De Laval... . . 193

Standards, Federal. . . . . . . . . 365

— feeding................ 48

- for cream................ 148

- legal. . . . . . . . . . . . . . . 365

- legal milk. . . . . . . . . . . . . 145

- State for milk and cream....... 368

"Standing up" of butter.......... 196

Starters................ 198, 245

Starters, "mother" ... . . . . . . . . 202

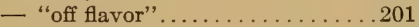

- preparation of ............ 354

"State brands" for cheese.... . . . . 345

State standards for milk and

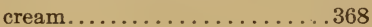

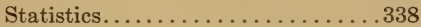

Stearic acid.................. 22

Stearin.................... 22

Sterile milk. . . . . . . . . . . . . . 109

Sterilization. . . . . . . . . 130, 143 
PAGE

Sterilization of milk ...........121

Sterilized milk, Federal standard

for . . . . . . . . . . . . . 365

Stilton cheese............... . 280

Stimulants and aromatics....... 50

Stirred curd cheese... . . . . . . . . 270

Storch mentioned. . . . . . . . . . . 199

Strainers.................... 137

Straining milk. . . . . . . . . . . . . 137

Strawberry ice cream. . . . . . . . . 310

Streaked butter......... . . . . 226

Strippings................. 30

Subearth duct for curing rooms. . . . 334

Succulent foods, necessity for..... 50

Sugar in milk............... 25

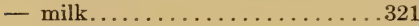

- - formation of ........... 9

Sulphuric acid. . . . . . . . . . . . . 104

Sunlight, effect on cleanliness. .... 136

Surface tension............23, 163

Sweet cream butter............. 207

- curdling................... 113

Sweetened condensed milk.........316

— — Federal standard for. . . . . 366

"Swelling" of cream............218

Swiss cattle, Brown........... 73

Swiss cheese............ . 279, 289

- - imitation ... . . . . . . . . . 279

System, butter and cheese factory...341

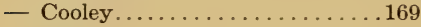

- gathered cream...............341

- metric.................. 363

Table butter worker ... . . . . . . . 224 Tablets, Farrington's alkaline.205, 351

Taints, detection of . . . . . . . . . 357

Teat................... 4

Teats, rudimentary in bulls....... 56

Temperature, best for churning. . . 211

- correction for lactometer... 81, 350

- effect of an activity of rennet. . 247

- - - churning............211

- for deep setting creaming......169

- for ripening cheese...........264

- - - cream............... 203

- shallow pan creaming...... 167

- washing butter...........221

- of reading fat in Babcock test...............95, 106

- optimum................ 110

- relation of to centrifugal separation................... 182

- - to churning.........211
PAGE

Temperature, rise in churning. . . .219

Test, Babcock. ............ 91, 94

— - calibration of glassware... . .359

— - for butter or cheese..........359

- Beimling...................92

- bottle Babcock.............. 96

- - B. and W.............. 96

- - cream................ 96

- - detachable neck.......... 96

— - double necked. . . . . . . . . . 96

- - Ohlssen ............... 96

- - skimmed milk........... 96

- butyrometer............... 93

- churn oil................. 84

- Cochran................... 91

- Cornell butter moisture. . . . . . . 359

— Failyer and Willard' .... . . . . . 89

- for colostrum............... 18

- Gerber's.................... 92

- history of milk ............ 36

- hot iron................. . . 358

- Iowa Station............... 90

- litmus. . . . . . . . . . . . . . . . 351

- Mann's acid. . . . . . . . . . . . . . 204

- Marschall rennet. . . . . . . . . . . 358

- Monrad rennet. . . . . . . . . . . . .358

- Parsons'... . . . . . . . . . . . . . . . 90

- phenolphthalein... . . . . . . . . 351

- rennet... . . . . . . . . . . . . 243, 358

- Short's... . . . . . . . . . . . . . . 89

- Wisconsin curd. ........ . . . 357

Testing Babcock glassware. . . . . . . 359

- co-operative cow.......... 47

- of milk............... 77

Tests, acid................. 204

— and rules... . . . . . . . . . . . . . 349

Texture of butter..........222, 230

_- relation of wash water to 222

- of cheese............... 268

Theory of centrifugal separator. . . 180

Thermometer, centigrade ... . . . . 349

— Farhenheit. . . . . . . . . . . . . . . . 349

- scales, comparison of . . . . . . . 349

Three-quarter blood, definition.... 53

- bloods, variation in.........61 61

Tiemann, quoted.............. 197

Tinware, how to clean.......... 136

— ironclad................. . 135

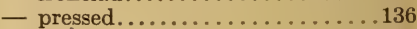

- rusty.................... 136

Tissue, connective............ 3

- fatty................ 3

Titration of media............. 128 
Total solids, determination of..... 77 .

- - formulæ for ............350

Truckle cheese................273

Tubercle bacillus............ 122

Tubular separator.............188

Turbine steam Babcock.......... 95

Tutti frutti ice eream............ 311

Tyrotoxicon.................115

Udder..................2, 43

- caked.................. 38

Ultimate follicles............ 3,6

United States separator. .187, 191, 193

Urea....................... 26

Useful rules and tests. . . . . . . . . . . 349

Utensils care of milk............160

Van Slyke, quoted.......31, 235, 240

Varieties of cheese.............269

Variation in three-quarter bloods... 61

Variations in quality of milk..... 27

Vaudin, quoted............. 18

Vaughn, quoted...............115

Veins, milk................ 56

Vertical butter worker...........225

Vessels cleaning................ 119

Victoria separator.............. 191

Virgin animals, milk from........ 10

Visco-cream................147

Viscogen.................147

- preparation of .............352

viscosity ..................164

— of milk................210

— - - relation of to churning...2210

- - - - creaming.........167
PAGE

Volatile fats.............20, 197

- - source of............... 21

Von Klenze, quoted. ..........269

Walnut ice cream. ............ 310

- parfait........................ 310

Wash water, relation of, to butter

flavor................223

- - - - texture of butter. .222

Washing butter..............220

- curds................272

- tinware.................. 136

Water ices.................. . 300

- necessity of in ration......... 50

- supply of creameries........... 331

Wedge shape form .............. 42

Wells..................... 331

Wensleydale cheese.........280, 289

"Wet" cheese..................271

Whey..................... 315

— cheese..................322

- Federal standards for..........367

Wheyn....................... 324

Whey, removal of .............249

White specks in butter.........208

Willard, Professor, mentioned..... 89

Williams, Jesse, mentioned........ 341

Wiltshire cheese................ 280

Wire strainers, size of . . . . . . . 137

Wisconsin curd test............. 357

Woll, F. W., quoted........... 94

Working butter..............223

Yeasts.....................109

Young America cheese..........273 

The following pages contain advertisements of books on kindred subjects 



\section{Dairy Cattle and Milk Production \\ By CLARENCE H. ECKLES \\ Illustrated, cloth, $12 m o, \$ 1.60$ net; postpaid, $\$ 1.73$.}

The author has brought together from widely scattered sources all the information necessary to acquaint the student or farmer with the principles he must understand and practise in order to be successful with dairy cattle. He takes up all of the dairy breeds, their characteristics and adaptations. The selection of the individual cow, calf-raising, management of the cow, breeding, stable construction, as well as a consideration of the ailments of cattle, likewise come in for detailed treatment.

The book will render great assistance to the practical farmer interested in dairy cattle, who will find the material presented here in such a way that it will assist him to care properly for his animals and to produce milk economically. For fifteen years Professor Eckles has had charge of a herd of from thirty to fifty cows, including all the leading dairy breeds. For the past ten years he has been teaching in the University of Missouri where he is Professor of Dairy Husbandry. His combined experiences have well fitted him for the task of writing the book on dairy cattle and milk production.

\section{Milk: Its Nature and Composition}

\section{By C. M. AIKMAN}

Cloth, 180 pages, illustrated, 12 mo, \$1.25 net; by mail, \$1.34.

A handbook on the chemistry and bacteriology of milk, butter and cheese. It treats of the causes and conditions influencing the quality and quantity of milk, of rennet and its action, and of the importance of bacteria for butter and in cheese making.

\section{The Farm and the Dairy}

\section{By J. P. SHELDON}

Fourth revised edition, cloth, 158 pages, illustrated, $12 \mathrm{mo}, \$ 1.00$ net; by mail, $\$ 1.08$.

A valuable text-book on practical dairying, detailing the most modern developments in dairy farming.

\section{Dairy Chemistry}

\section{By HARRY SNYDER}

Cloth, 190 pages, illustrated, 12 mo, $\$ 1.00$ net; by mail, \$1.12.

This book incorporates the results of the more important recent investigations in dairying, milk tests, etc. A chapter treats of the influence of different foods upon the quality of milk and dairy products, and on the rational feeding of dairy stock, while in an Appendix are given tables of the composition of fodder and feeding stuffs, and tables for the correction of lactometer readings.

\section{THE MACMILLAN COMPANY}

\section{PUBLISHERS \\ 64-66 Fifth Avenue \\ NEW YORK}




\title{
Sheep Farming
}

\author{
By JOHN A. CRAIG and F. R. MARSHALL
}

Illustrated. Cloth, 12mo. \$1.50 net.

This book deals with sheep husbandry as a phase of intensive farming. Recognizing that it is likely to be used by persons unfamiliar with sheep, the authors have worked from the standpoint of the producer of market stock, rather than from the standpoint of the professional breeder. The various breeds are discussed in such a way as to enable the reader to select the kind that is most likely to do well under his conditions and to acquaint him with the care it is accustomed to and needs. The management of the flock in the fall, winter, spring and summer seasons, the formation of the flock, the selection of foundation stock, and the means of maintaining a high standard of flock efficiency, are all discussed in subsequent chapters.

\section{Forage Crops for the South}

\section{By S. M. TRACY}

Illustrated. Cloth, 12mo. Preparing.

Professor Tracy has had long experience in Southern agriculture, both in application and in teaching. He was formerly Professor of Agriculture in the Mississippi Agricultural College, and now conducts a branch station or farm for the United States Department of Agriculture. He is a botanist of note and has traveled extensively in the South as a collector. His book is not only authentic, but practical. In it is contained a discussion of all kinds of plants and crops adapted to the Southern States for fodder, soiling, pasturing and hay. The text is abundantly illustrated.

\section{THE MACMILLAN COMPANY}

\section{PUBLISHERS \\ 64-66 Fifth Avenue \\ NEW YORK}




\title{
NEW FARM AND GARDEN BOOKS
}

\section{Injurious Insects: How to Recognize and Control Them}

\author{
By W. C. O'KANE
}

Entomologist of the New Hampshire Agricultural Experiment Station, and Professor of Economic Entomology in New Hampshire College

Illustrated. Cloth, 12mo. \$2.00 net.

Complete information on the characteristics, life histories and means of control of the more common injurious insects, including those infesting field crops, vegetables, fruits, the principal pests of domestic animals, stored products and the household, is contained in this book. A distinctive feature of the work is the illustrations with which the text throughout is accompanied. These have been made especially for Dr. O'Kane. With each insect treated he shows in an original photograph the characteristic injurious stage or the typical work of the insect where that is characteristic. By this means the author hopes that the layman will be able to recognize an insect that threatens by the picture aside from any description in the text.

\section{Principles of Fruit Growing}

\section{By Professor L. H. BAILEY}

New edition. Cloth, 12 mo. $\$ 1.50$ net. Since the original publication of this book, in 1897, it has gone through many editions. The progress of fruit growing in the meantime has been very marked and it has been necessary to completely rewrite the work. The present issue of it brings the accounts of the new practices and discoveries as they relate to fruit growing up to date. All of the text and practically all of the illustrations are new.

\section{THE MACMILLAN COMPANY PUBLISHERS




\title{
How to Keep Hens for Profit
}

\author{
By C. S. VALENTINE
}

Cloth, illustrated, $12 \mathrm{mo}, \$ 1.50$ net; postpaid, $\$ 1.63$

"The Plymouth Rock, Java, Dominique, Wyandotte, Rhode Island Red, and Buckeye breeds are discussed in the first few chapters. Considerable attention is given to other breeds later on. Eighteen beautiful half-tone engravings adorn the book. From the standpoint of the practical farmer and poultry-grower, we consider this book as one of the very best of its kind. The author is evidently an experienced poultryman. It is a book that should be of special help to beginners in poultry, while at the same time it contains much information for the expert."

-Farmers' Tribune.

\section{The Beginner in Poultry}

\section{By C. S. VALENTINE}

Decorated Cloth, profusely illustrated, $12 \mathrm{mo}, \$ 1.50$ net; postpaid, $\$ 1.63$

It has been estimated that of the five million people who are raising poultry in this country today half have gone at it blindly. And it is just as impossible to make a success of the poultry business without preparation as it is impossible to succeed in any other business without an acquaintance with the fundamentals. The difficulty which the novice has experienced in going at the raising of chickens systematically in the past has been that he could find no book in which the essentialsonly the essentials and all of them-of poultry-raising are given. To write such a book has been Mr. Valentine's purpose. In "The Beginner in Poultry" he discusses the different breeds of fowls, the types of houses, feeding and the kinds of food, raising chickens for the market and for their eggs, diseases and their cures and everything else which will be of value for the one who is starting out-and much for the seasoned poultry-raiser as well.

\section{THE MACMILLAN COMPANY}

\section{PUBLISHERS}

64-66 Fifth Avenue

NEW YORK 


\title{
THE RURAL OUTLOOK SET
}

\author{
By Professor L. H. BAILEY
}

Director of the New York State College of Agriculture at Cornell University

Four Volumes. Each, cloth, 12 mo. Uniform binding, attractively boxed. $\$ 5.00$ net per set; carriage extra. Each volume also sold separately.

In this set are included three of Professor Bailey's most popular books as well as a hitherto unpublished one, " "The Country-Life Movement." The long and persistent demand for a uniform edition of these little classics is answered with the publication of this attractive series.

\section{The Country-Life Movement}

Cloth, 12 mo, 220 pages, $\$ 1.25$ net; by mail, \$1.34

This hitherto unpublished volume deals with the present movement for the redirection of rural civilization, discussing the real country-life problem as distinguished from the city problem, known as the back-to-the-land movement.

\section{The Outlook to Nature (New and Revised Edition)}

Cloth, 12mo, 195 pages, $\$ 1.25$ net; by mail, \$1.34

In this alive and bracing book, full of suggestion and encouragement, Professor Bailey argues the importance of contact with nature, a sympathetic attitude toward which "means greater efficiency, hopefulness, and repose."

\section{The State and the Farmer (New Edition)}

Cloth, $12 m o, \$ 1.25$ net; by mail, $\$ 1.34$

It is the relation of the farmer to the government that Professor Bailey here discusses in its varying aspects. He deals specifically with the change in agricultural methods, in the shifting of the geographical centers of farming in the United States, and in the growth of agricultural institutions.

\section{The Nature Study Idea (New Edition)}

Cloth, 12 mo, \$1.25 net; by mail, $\$ 1.34$

"It would be well," the critic of The Tribune Farmer once wrote, "if "The Nature Study Idea' were in the hands of every person who favors nature study in the public schools, of every one who is opposed to it, and, most important, of every one who teaches it or thinks he does." It has been Professor Bailey's purpose to interpret the new school movement to put the young into relation and sumpathy with nature,-a purpose which he has admirably accomplished.

\section{THE MACMILLAN COMPANY}




\section{RURAL SCIENCE SERIES}

\section{Edited by L. H. BAILEY}

On Selection of Land, etc.

Isaac P. Roberts' The Farmstead . . . . , $\$ 150$

On Tillage, etc.

F. H. King's The Soil

* 150

Isaac P. Roberts' The Fertility of the Land • • • 150

F. H. King's Irrigation and Drainage . . . . : 150

Edward B. Voorhees' Fertilizers . . • • • • 125

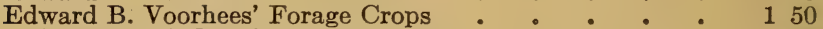

J. A. Widtsoe's Dry Farming . • • • • • • 150

L. H. Bailey's Principles of Agriculture : : : : 125

On Plant Diseases, etc.

E. C. Lodeman's The Spraying of Plants . • - . 125

On Garden-Making

L. H. Bailey's Garden-Making ․ • • • - 150

L. H. Bailey's Vegetable-Gardening : $: \quad$ : $\quad$ : 150

L. H. Bailey's Forcing Book • • • : ? : 125

On Fruit-Growing, etc.

L. H. Bailey's Nursery Book • • • • • 150

L. H. Bailey's Fruit-Growing . • • • • . 150

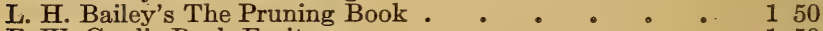

F. W. Card's Bush Fruits • : • : : : 150

On the Care of Live-stock

Nelson S. Mayo's The Diseases of Animals . • • . 150

W. H. Jordan's The Feeding of Animals : • : . 150

I. P. Roberts' The Horse . • • • • • . 125

M. W. Harper's Breaking and Training of Horses : : 150

George C. Watson's Farm Poultry. . • . • . 125

On Dairy Work, Farm Chemistry, etc.

Henry H. Wing's Milk and Its Products . . . 。 150

J. G. Lipman's Bacteria and Country Life . . . . 150

On Economics and Organization

I. P. Roberts' The Farmer's Business Handbook . . . 125

George T. Fairchild's Rural Wealth and Welfare : $\quad 125$

H. N. Ogden's Rural Hygiene. ‥ . . . • 150

J. Green's Law for the American Farmer : $:$ : 150

\section{THE MACMILLAN COMPANY}




\title{
THE RURAL MANUALS
}

\author{
Edited by L. H. BAILEY
}

\section{Manual of Farm Animals}

A Practical Guide to the Choosing, Breeding and Keep of Horses, Cattle, Sheep and Swine.

\section{By MERRITT W. HARPER}

Assistant Professor of Animal Husbandry in the New York State College of Agriculture at Cornell University

Illustrated, decorated cloth, 12mo, 545 pages, index, $\$ 2.00$ net; by mail, \$2.18

"The work is invaluable as a practical guide in raising farm animals."

-Morning Telegram.

"A book deserving of close study as well as being handy for 'reference, and should be in the possession of every farmer interested in stock."-Rural World.

\section{Manual of Gardening}

A Practical Guide to the Making of Home Grounds and the Growing of Flowers, Fruits and Vegetables for Home Use.

\section{By L. H. BAILEY}

Illustrated, cloth, $12 \mathrm{mo}, 544$ pages, $\$ 2.00$ net; by mail, \$2.17

This new work is a combination and revision of the main parts of two other books by the same author, "Garden Making" and "Practical Garden Book," together with much new material and the result of the experience of ten added years. Among the persons who collaborated in the preparation of the other two books, and whose contributions have been freely used in this one, are C. E. Hunn, a gardener of long experience; Professor Ernest Walker, reared as a commercial florist; Professor L. R. Taft, and Professor F. A. Waugh, well known for their studies and writings on horticultural subjects.

\section{A STANDARD WORK REVISED AND ENLARGED}

\section{The Farm and Garden Rule Book}

\section{By LIBERTY H. BAILEY}

Illustrated, cloth, 12mo, \$2.00 net

When Professor Bailey's "Horticulturist's Rule Book" was published nearly twenty-five years ago, the volume became a standard agricultural work running through sixteen editions. Taking this book as a basis the author has now made a wholly new book, extending it to cover the field of general farming, stock-raising, dairying, poultry-rearing, horticulture, gardening, forestry, and the like. It is essentially a small cyclopedia of ready rules and references packed full from cover to cover of condensed, meaty information and precepts on almost every leading subject connected with country life. 


\title{
Cyclopedia of American Agriculture
}

\author{
Edited by L. H. BAILEY \\ Director of the College of Agriculture and Professor of Rurai Economy,
Cornell University.
}

With 100 full-page plates and more than 2,000 illustrations in the text; four volumes; the set, $\$ 20.00$ net; half morocco, \$32.00 net; carriage extra

\section{VOLUME I-Farms \\ VoLUME III-Animals \\ VOLUME II-Crops \\ VoLUME IV-The Farm and the Community}

"Indispensable to public and reference libraries ... readily comprehensible to any person of average education."-The Nation.

"The completest existing thesaurus of up-to-date facts and opinions on modern agricultural methods. It is safe to say that many years must pass before it can be surpassed in comprehensiveness, accuracy, practical value, and mechanical excellence. It ought to be in every library in the country."-Record-Herald, Chicago.

\section{Cyclopedia of American Horticulture}

\author{
Edited by L. H. BAILEY \\ With over 2,800 original engravings; four volumes; the set, \\ \$20.00 net; half morocco, \$32.00 net; carriage extra
}

"This really monumental performance will take rank as a standard in its class. Illustrations and text are admirable. . . . Our own conviction is that while the future may bring forth amplified editions of the work, it will probably never be superseded. Recognizing its importance, the publishers have given it faultless form. The typography leaves nothing to be desired, the paper is calculated to stand wear and tear, and the work is at once handsomely and attractively bound."-New York Daily Tribune.

\section{THE MACMILLAN COMPANY}

\section{PUBLISHERS \\ 64-66 Fifth Avenue \\ NEW YORK}



LAN $16 \quad 1913$ 




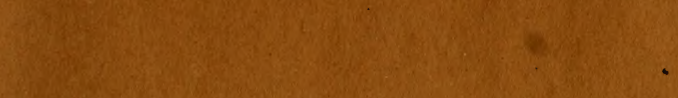
and

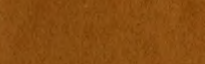

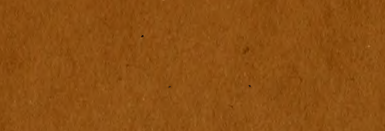

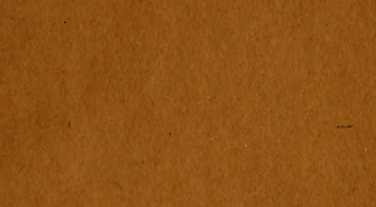

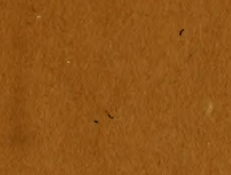

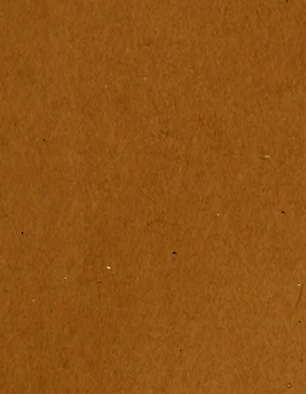

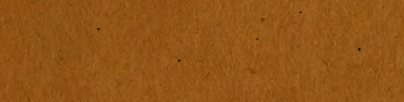






\section{LIBRARY OF CONGRESS ||||||||||||||||||||||||||||||||||||||||||||||||

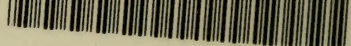 0000899941 ?}

